\title{
Measurement of Mixing and CP Violation in the Two-Body D0 decays to KK, pipi and Kpi with the BaBar Experiment
}

$\mathrm{PhD}$. Thesis

By Giulia Casarosa 
SLAC-R-1000

\title{
Università di Pisa
}

The School of Graduate Studies in Basic Sciences "Galileo Galilei" SSD FIS/01

\author{
PhD. Thesis
}

Measurement of Mixing and $C P$ Violation in the Two-Body $D^{0}$ decays to $K^{+} K^{-}, \pi^{+} \pi^{-}$ and $K^{ \pm} \pi^{\mp}$ with the BABAR Experiment

Candidate

Giulia Casarosa
Supervisor

Prof. Francesco Forti 

a mamma, babbo e clau 



\section{Acknowledgments}

First and foremost I wish to thank Francesco Forti. He has been a great guide through these years, supporting my scientific interests but also suggesting new possible ones. I'm very grateful for the countless opportunities I had to learn new things, face different problems, discover a little bit more of the (physics) world. His support has sometimes gone beyond the physics, I can't thank him enough for this.

I wish to thank the people of the BABAR and SuperB group in Pisa. Everybody always found time for me and taught me something new. In particular I am very grateful to Eugenio Paoloni. His incredible kindness and helpfulness to explore any little (or big) doubt, concern or simple curiosity have contributed to grow my love for physics.

I also wish to thank the people of the BABAR charm AWG, in particular Nicola Neri and Bill Dunwoodie, always ready to help with their invaluable experience. Their critical view of the analysis has increased the quality of the measurement. Bill Lockman and Kevin Flood have played a fundamental role in this measurement with their suggestions, comments, and technical support.

I want to thank the people at SLAC, Tina, Isabella, Antonio, Pietro and David, a big family that made the six months I spent there a wonderful time and an indelible memory.

My family, to whom this thesis is dedicated, is my rock.

Giulia 



\section{Contents}

Contents $\quad$ v

$\begin{array}{ll}\text { Introduction } & 1\end{array}$

1 Mixing and $C P$ Violation in the Standard Model 5

1.1 The Standard Model Today . . . . . . . . . . . . . . . . . . . . 5

1.2 Neutral Meson Mixing . . . . . . . . . . . . . . . . . . . . . . . 14

1.2.1 Time Evolution of the Flavor Eigenstates . . . . . . . . . . . 14

$1.2 .2 \quad C P$ Violation . . . . . . . . . . . . . . . . . . . . . 19

1.2.3 Mixing Phenomenology in the Standard Model . . . . . . . . . . . 22

1.3 Mixing and $C P$ Violation with the Lifetime Ratio in $D^{0}$ Decays . . . . . . 25

1.4 Experimental Status of the Observables . . . . . . . . . . . . 27

2 The BABAR Experiment at the B-Factory PEP-II 31

2.1 The PEP-II B Factory . . . . . . . . . . . . . . . . . . . . . . 31

$2.1 .1 \quad$ PEP-II Layout . . . . . . . . . . . . . . . . . . . . . 33

2.1.2 PEP-II Performances . . . . . . . . . . . . . . . . . . . 34

$2.1 .3 \quad$ PEP-II Background . . . . . . . . . . . . . . . . . 35

2.2 Overview of the BABAR Detector $\ldots \ldots \ldots \ldots \ldots$

2.3 The Silicon Vertex Tracker $(\mathrm{SVT}) \quad \ldots \ldots \ldots$. . . . . . . . . . 40

2.3 .1 Detector Layout . . . . . . . . . . . . . . . . . 40

2.3.2 Detector Performance . . . . . . . . . . . . . . . . . 42

2.4 The Drift CHamber $(\mathrm{DCH}) \ldots \ldots \ldots \ldots \ldots$

2.4 .1 Detector Layout . . . . . . . . . . . . . . . . . 43

2.4.2 Detector Performance . . . . . . . . . . . . . . . . 45 
2.5 The Cherenkov Light Detector . . . . . . . . . . . . . . . . . . 47

2.5.1 Detector Layout . . . . . . . . . . . . . . . 47

2.5.2 Detector Performance . . . . . . . . . . . . . . . . . . 49

2.6 The ElectroMagnetic Calorimeter $(\mathrm{EMC}) \ldots \ldots \ldots \ldots$

2.6 .1 Detector Layout . . . . . . . . . . . . . . . 5 53

2.6.2 Detector Performance . . . . . . . . . . . . . . . . 54

2.7 The Instrumented Flux Return (IFR) $\ldots \ldots \ldots \ldots$

2.7 .1 Detector Layout . . . . . . . . . . . . . . . 56 56

2.7.2 Detector Performance . . . . . . . . . . . . . . . . . 59

2.8 The BABAR Trigger . . . . . . . . . . . . . . . . . . . . 62

2.9 Monte Carlo Production and Data Flow . . . . . . . . . . . . . . 63

3 Lifetime Ratio Analysis Overview $\quad 65$

3.1 General Outlook . . . . . . . . . . . . . . . . . . 65

3.1 .1 Reconstructed Signal Channels . . . . . . . . . . . . . . . 67

3.1.2 Proper Time Measurement . . . . . . . . . . . . . . . . 68

3.1.3 Mass and Proper Time Correlation of the Signal Candidates . . . . 68

3.1.4 Backgrounds . . . . . . . . . . . . . . . . . 70

3.1 .5 Simultaneous Fit . . . . . . . . . . . . . 70

3.2 Previous BABAR Analyses ． . . . . . . . . . . . . . . . . . . . 72

3.3 Analysis Improvements and Optimizations . . . . . . . . . . . . . . . . . 74

$\begin{array}{lll}4 & \text { Event Selection } & 77\end{array}$

4.1 Data and Monte Carlo Samples . . . . . . . . . . . . . . . . 77

4.2 Reconstruction and Selection Criteria _. . . . . . . . . . . . 79

4.2.1 Skim and Reskim Selections . . . . . . . . . . . . . . . . 80

$4.2 .2 \quad$ Final Selection . . . . . . . . . . . . . . . . . . . . 81

4.3 Signal and Sidebands Regions Definition . . . . . . . . . . . . . . 82

4.3 .1 Mass Fits . . . . . . . . . . . . . . . . 83

4.3.2 Signal Region Optimization . . . . . . . . . . . . . . . . 85

4.4 Expected Number of Signal and Background Candidates . . . . . . . . . . . 90

$5 \quad$ Measurement of $y_{C P}$ and $\Delta Y \quad 93$ 
5.1 Characterization of the Signal Events . . . . . . . . . . . . . . . . 93

5.1 .1 Parameterization . . . . . . . . . . . . . . . . 97

5.1.2 Simultaneous Fit to the Signal-Only MC Datasets . . . . . . . . 100

5.2 Characterization of the Misreconstructed-Charm Events . . . . . . . . . . . 102

5.2 .1 Parameterization . . . . . . . . . . . . . . . . . . 104

5.3 Characterization of the Combinatorial Events . . . . . . . . . . . . 106

5.3 .1 Parameterization . . . . . . . . . . . . . . . 106

5.4 Proper Time Error PDF . . . . . . . . . . . . . . . . . . . . 113

5.5 Simultaneous Fit . . . . . . . . . . . . . . . . . . . 115

$5.5 .1 \quad$ Fit Strategy . . . . . . . . . . . . . . . . . . . . 116

5.5.2 Validation of the Fit on the Simulated Events . . . . . . . . . . 117

5.5 .3 Data Results . . . . . . . . . . . . . . . . . . . . 119

6 Crosschecks and Evaluation of the Systematic Uncertainty 123

6.1 Data Crosschecks . . . . . . . . . . . . . . . . . . . . . 123

6.1.1 Tagged-Only and Untagged-Only Fits . . . . . . . . . . . . . 124

6.1.2 Signal PDF Configurations . . . . . . . . . . . . . . . . . . . . 124

6.1.3 Effect of the Direct $C P$ Violation . . . . . . . . . . . . 127

6.1 .4 Detector Misalignment . . . . . . . . . . . . . . . 128

6.1.5 Additional Crosschecks . . . . . . . . . . . . . . . . . . . 132

6.2 Evaluation of the Systematic Uncertainty . . . . . . . . . . . . . . . 134

$6.2 .1 \quad$ Fit Region . . . . . . . . . . . . . . . . . . . . 134

$6.2 .2 \quad$ Signal PDF . . . . . . . . . . . . . . . . . . . . 136

6.2.3 Misreconstructed-Charm PDF and Yields . . . . . . . . . . . 138

6.2 .4 Combinatorial PDF and Yields . . . . . . . . . . . . 138

6.2 .5 Event Selection . . . . . . . . . . . . . . . . . . . . . . . 141

6.2 .6 Systematic Summary . . . . . . . . . . . . . . . . . . . 142

6.3 Compatibility with the Previous BABAR Results . . . . . . . . . . . . . . 142

$\begin{array}{lll}7 & \text { Conclusions } & 147\end{array}$

$7.1 \quad$ Final Result . . . . . . . . . . . . . . . . . . . . . . . 147

7.2 Future Perspectives . . . . . . . . . . . . . . . . . . 150 
A The Area of the Unitary Triangles

B Comparison Data - MC

C Mass Fits Parameters

D Signal Region Optimization Studies

D.1 Asymmetry as a Function of the Signal Region Width . . . . . . . . . 163

D.2 Width Optimization . . . . . . . . . . . . . . . . 164

E Distributions of the Events in the Three Categories 167

E.1 Signal Events Distributions _ . . . . . . . . . . . . . . . . 167

E.2 Misreconstructed-Charm Events Distributions . . . . . . . . . . . . . . 170

E.3 Combinatorial Events Distributions . . . . . . . . . . . . . . . . . 173

$\begin{array}{lll}\text { F Complete Fit Results } & 177\end{array}$

$\begin{array}{ll}\text { Bibliography } & 181\end{array}$ 


\section{Introduction}

After the discovery of strange particles in cosmic rays during the years after World War II, the idea of associated production was introduced by Pais (1953) to explain the copious production versus the long lifetime of these particles. In the same year, at the Bagnèresde-Bigorre Conference, scientists from all over the world had the opportunity to compare their cloud chambers results and the order emerged from chaos with a first classification of these new states. They agreed that two states correspond to different particles if they have different mass. In the following two years the basic properties of these particles where known and the accelerators invaded the scene, allowing a great increase of statistics. In 1955 Gell-Mann and Nishijima independently wrote the relation between the third component of the isospin $I_{3}$, the charge $Q$, the baryon number $B$ and a new quantum number $S$, the strangeness, $Q=I_{3}+(S+B) / 2$. Heisenberg's idea of isospin was extended to the new particles just two years before, by Gell-Mann. Among the new strange particles there was one, the $\theta^{0}$ (now known as $K^{0}$ ) that differed from its antiparticle. The value of the strangeness $S$ was +1 for the former and -1 for the latter. It was also clear that the processes $K^{0} \rightarrow \pi^{+} \pi^{-}$and $\bar{K}^{0} \rightarrow \pi^{+} \pi^{-}$were both possible, and therefore also $K^{0} \rightarrow \pi^{+} \pi^{-} \rightarrow \bar{K}^{0}$. Gell-Mann and Pais [1] suggested that the $K^{0}$ was not a physical state with well-defined mass and lifetime but it was a particle mixture of the physical states $K_{1}$ and $K_{2}: K_{1,2}=\left(K^{0} \pm \bar{K}^{0}\right) / \sqrt{2}$. The particle that was actually observed was the $K_{1}$ being $C$-even as the $\pi^{+} \pi^{-}$state in which it decayed. They predicted the existence of a second particle, not yet observed, the $K_{2}$, corresponding to the orthogonal mixture which should have a different lifetime and should decay into a $C$-odd state, such as three pions. One year after its prediction, this particle was discovered [2] and meson mixing was experimentally established. We now know that $K_{1,2}$ are not the physical states but they are the $C P$ eigenstates, and the physical states are $K_{S}^{0}$ and $K_{L}^{0}$, but this was not known until $C P$ violation $(C P V)$ was discovered in 1964.

$P$ violation discovery in weak interaction was a consequence of the so-called $\theta-\tau$ puzzle, involving again strange particles. Two particles with the same mass were observed decaying in final states with opposite parities: $\theta^{+} \rightarrow \pi^{+} \pi^{0}$ and $\tau^{+} \rightarrow \pi^{+} \pi^{-} \pi^{+}$. Either the two were different particles or parity was violated in their decay. At the $6^{\text {th }}$ Rochester Conference, in 1956, it was suggested that there was no experiment proving that parity was conserved in weak decays. In a paper by Lee and Yang [3] a certain number of ex- 
periments to test the conservation of $P$ in weak interactions were proposed, and promptly realized $[4,5]$ one year later, in 1957. In the same year, Lee, Oehme and Yang [6], showed that the observed $P$ violation implied also a $C$ violation, but the combined operation $C P$ was still a symmetry. The fact that the exchange of left and right was not a symmetry operation for the weak interactions was quite a disturbing and hard to believe truth for the physics community of that time. This uneasiness was mitigated by the fact that the symmetry was actually preserved if particles were exchanged with antiparticles, in other words, exchanging left with right and going into the anti-world, the symmetry holds.

The discovery of $P$ and $C$ violations in weak interactions was a key ingredient for Feynmann and Gell-Mann, independently, to write the V-A theory that is at the basis of the Standard Model (SM).

In 1964 an experiment designed by Cronin, Fitch, Christenson and Turlay [7] to primarily study $K^{0}$ regeneration discovered that around $2 \%$ of the $K_{L}$ decays were to $\pi^{+} \pi^{-}$, which is an indication of $C P$ violation. If $P$ and $C$ were maximally violated, $C P$ was minimally violated and various theoretical model were proposed in order to accommodate this in the Standard Model.

The discovery of $C P V$ had consequences also beyond the elementary particle theory, in 1967 Sakharov proposed a model which could explain why the universe consists essentially of matter with very little antimatter, although equal amounts of both are probably created at a very early stage (baryogenesis). One of the three Sakharov conditions was $C P$ violation.

After 1964 a certain number of models were proposed in order to accommodate $C P V$ in the theory. Wolfenstein proposed the superweak model [8] in which $C P V$ was due to very weak $\Delta S=2$ four-fermion interaction. This model was ruled out in 1988 by the observation of direct $C P V$ in $K^{0}$ decays by NA31 [9], confirmed by NA48 [10] and KTeV [11] 11 years later. In 1973 Kobayashi and Maskawa [12] extended the $2 \times 2$ Cabibbo quark matrix mixing to a $3 \times 3$ matrix, now called Cabibbo-Kobayashi-Maskawa (CKM) matrix, within a six-quark model. $C P$ violation was naturally included, originating from the irreducible complex phase needed in the parameterization of the matrix. It is worth noticing that in 1973 the charm quark was not yet discovered, although it was predicted by Glashow Iliopulos and Maiani who introduced it to explain the suppression of flavor changing neutral currents (GIM mechanism). An indication of the charm quark mass was computed [13] from the $K^{0}-\bar{K}^{0}$ mixing amplitude. The charm quark was actually discovered in 1974 by BNL and SLAC in the $c \bar{c}$ state $J / \psi$.

In 1999 the key ingredients of the current Standard Model were all there. Although the CKM mechanism was the most probable explanation for $C P V$ in the SM, it had to be confirmed in other systems. This mission was accomplished by the B-Factories BABAR and Belle that, in their years of data taking, produced measurements showing the consistency of the CKM picture. These measurements were conducted on the $B^{0}-\bar{B}^{0}$ mixing system, the two mesons were produced in an entangle state that allowed precision measurements 
of mixing and $C P V$ parameters. Beside the confirmation of the CKM mechanism, studies of $B^{0}-\bar{B}^{0}$ mixing, first observed in 1987 the ARGUS [14] and UA1 [15] Collaborations, also provided an indication on the mass of the top quark.

In 2006 the mixing in $B_{s}-\bar{B}_{s}$ system was observed [16], completing the picture of the mixing systems in the down-quark sector and confirming again SM predictions.

The first evidence of $D^{0}-\bar{D}^{0}$ mixing was reported by the BABAR [17] and Belle [18] Collaborations in 2007, and confirmed one year later by the CDF Collaboration [19]. Although combining all the measurements together the significance for mixing exceeds the 10 standard deviations, there is not a single measurement that could establish mixing with a significance of at least 5 standard deviations. This is due to the fact that the mixing in the $D^{0}-\bar{D}^{0}$ system is very small, $\mathcal{O}(<1 \%)$.

In November 2011 the first evidence of $C P V$ in the $D^{0}-\bar{D}^{0}$ system was reported by the LHCb [20] and then CDF [19] Collaborations. Subsequent measurements by Belle, BABAR and an improved measurement by $\mathrm{CDF}$ set the present significance of $C P V$ to almost 5 standard deviations, combining all the measurements together. The SM predictions on $C P V$ (and mixing) for the charm sector are affected by large theoretical uncertainties and the interpretation of these results is not straightforward, there is a possibility that this could be a manifestation of physics beyond the Standard Model, but a SM explanation is not ruled out.

History showed that mixing systems represent an interesting and fertile environment where critical phenomena for the SM development have been observed, studied and then understood. The evidence of $C P V$ in charm could be one of these, but in order to solve this puzzle, an effort on both experimental measurements and theoretical computation should be pursued.

In this thesis we present the measurement of $D^{0}-\bar{D}^{0}$ mixing parameter $y_{C P}$ using the full $B A B A R$ data sample. We also searched for $C P$ violation in the $D^{0} \rightarrow K^{+} K^{-}, \pi^{+} \pi^{-}$ channels, finding the parameter $\Delta Y$ compatible with zero. In Chapter 1 , we briefly review the SM and introduce the theoretical framework of neutral meson mixing and $C P$ violation. The BABAR detector and the performance of each sub-detector are described in Chapter 2. In Chapter 3, we present an overview of the analysis including a brief description of the previous similar BABAR analyses and the expected improvements in the present analysis. The candidate reconstruction and selection are described in Chapter 4, together with the optimization of the signal region. The signal and background event classes are described in Chapter 5, where we also provide the probability density functions used to extract the mixing and $C P$ violating parameter. In Chapter 6 we describe the various crosschecks performed to validate the analysis and the evaluation of the systematic error. Finally in Chapter 7 we present the final results and their interpretation. 



\section{Chapter 1}

\section{Mixing and $C P$ Violation in the Standard Model}

We begin this chapter reviewing the SM sectors most relevant to this work. We introduce the mixing and $C P$ violation formalism, and present the time evolution of the flavor eigenstates. We briefly discuss the phenomenology of mixing for the different SM systems, commenting on the difficulties of computing the SM predictions for the $D^{0}$ system. We also present and discuss the observables, $y_{C P}$ and $\Delta Y$. Finally we show the experimental status of the mixing and $C P V$ observables in the charm sector.

\subsection{The Standard Model Today}

The Standard Model is a field theory describing the strong, weak and electromagnetic interactions in terms of elementary fermions (leptons and quark) interacting through the exchange of vector bosons, the forces mediators.

At present the known elementary particles are:

- six leptons (and their antiparticles) organized in three families:

$$
\left(\begin{array}{c}
\nu_{e} \\
e
\end{array}\right),\left(\begin{array}{c}
\nu_{\mu} \\
\mu
\end{array}\right),\left(\begin{array}{c}
\nu_{\tau} \\
\tau
\end{array}\right)
$$

- six quark flavors (and their antiparticles) organized in three families:

$$
\left(\begin{array}{l}
u \\
d
\end{array}\right),\left(\begin{array}{l}
c \\
s
\end{array}\right),\left(\begin{array}{l}
t \\
b
\end{array}\right)
$$

- the photon $\gamma$ : the boson mediator of the electromagnetic interaction;

- $W^{+}, W^{-}, Z^{0}$ : the three boson mediators of the weak interaction; 
- gluons: eight boson mediators of the strong interaction;

- the Higgs boson, $H$, responsible for the mass generation of the bosons and fermions. The first experimental observation of this boson has been recently reported by the ATLAS [21] and CMS [22] Collaborations.

The $\mathrm{SM}$ is based on the gauge symmetry group $S U(3)_{C} \otimes S U(2)_{I} \otimes U(1)_{Y}$, where $S U(3)_{C}$ describes the color symmetry of the strong interactions, $S U(2)_{I}$ the weak isospin and $U(1)_{Y}$ the hypercharge symmetry. Quarks and leptons (massless) are the irreducible transformations of the symmetry groups mentioned above and are organized in multiplets of defined chirality. For the first family we have:

- quarks:

$$
\begin{array}{r}
Q_{L}^{i n t}=\left(\begin{array}{c}
u_{L}^{i n t} \\
d_{L}^{i n t}
\end{array}\right) \rightarrow(\mathbf{3}, \mathbf{2})_{1 / 3} \\
u_{R}^{i n t} \rightarrow(\mathbf{3}, \mathbf{1})_{4 / 3}, \quad d_{R}^{i n t} \rightarrow(\mathbf{3}, \mathbf{1})_{-2 / 3}
\end{array}
$$

- leptons:

$$
\begin{array}{r}
L_{L}^{i n t}=\left(\begin{array}{c}
\nu_{L}^{i n t} \\
\ell_{L}^{i n t}
\end{array}\right) \rightarrow(\mathbf{1}, \mathbf{2})_{1 / 3} \\
\nu_{R}^{i n t} \rightarrow(\mathbf{1}, \mathbf{1})_{0}, \quad \ell_{R}^{i n t} \rightarrow(\mathbf{1}, \mathbf{1})_{-2}
\end{array}
$$

where:

- int stands for interaction eigenstates;

- $R$ (right) and $L$ (left) represent the chirality of the field: $\psi_{R / L}=\frac{1}{2}\left(1 \pm \gamma^{5}\right) \psi$;

- e.g. $(\mathbf{3}, \mathbf{2})_{1 / 3}$ means that a state is triplet under $S U(3)$ color group, a doublet in weak isospin, and the hypercharge is $1 / 3$;

We have included also the right handed neutrinos, of which only indirect evidence exists from neutrino mixing. The second and third families have the same representation.

We are not going to discuss the strong interaction sector, but we will focus on the $S U(2)_{I} \otimes U(1)_{Y}$ electroweak sector of the SM because it is the most relevant for this work.

Let's first fix the notation to describe elementary fermions and bosons: up-type quarks are the elements of the vector $U_{L, R}^{i n t}$, while down-type quarks of the vector $D_{L, R}^{i n t}$ :

$$
\begin{aligned}
& U_{L, R}^{i n t}=\left(\begin{array}{llll}
u_{L, R}^{i n t} & , & c_{L, R}^{i n t} \quad, & t_{L, R}^{i n t}
\end{array}\right)
\end{aligned}
$$

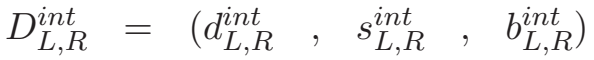


likewise for the leptons:

$$
\begin{aligned}
& \nu_{L, R}^{i n t}=\left(\begin{array}{lllll}
\nu_{e_{L, R}}^{i n t} & , & \nu_{\mu_{L, R}}^{i n t} & , & \nu_{\tau_{L, R}}^{i n t}
\end{array}\right) \\
& \ell_{L, R}^{i n t}=\left(\begin{array}{lllll}
e_{L, R}^{i n t} & , & \mu_{L, R}^{i n t} & , & \tau_{L, R}^{i n t}
\end{array}\right)
\end{aligned}
$$

The $S U(2)_{I}$ gauge fields are $\overrightarrow{\mathcal{W}}_{\mu}=\left(\mathcal{W}_{1 \mu}, \mathcal{W}_{2 \mu}, \mathcal{W}_{3 \mu}\right)$ while the only field of the $U(1)_{Y}$ group is $\mathcal{B}_{\mu}$.

The electroweak Lagrangian can be obtained by imposing the gauge symmetry $S U(2)_{I} \otimes U(1)_{Y}$ :

$$
\begin{aligned}
\mathcal{L}_{E W}= & -\frac{1}{4} \mathcal{W}_{\mu \nu} \mathcal{W}^{\mu \nu}-\frac{1}{4} \mathcal{B}_{\mu \nu} \mathcal{B}^{\mu \nu}+ \\
& i \sum_{k=1}^{3}\left\{\overline{U_{L_{k}}^{i n t}} \gamma_{\mu} \mathcal{D}^{\mu} U_{L_{k}}^{i n t}+\overline{U_{R_{k}}^{i n t}} \gamma_{\mu} \mathcal{D}^{\mu} U_{R_{k}}^{i n t}+\overline{D_{L_{k}}^{i n t}} \gamma_{\mu} \mathcal{D}^{\mu} D_{L_{k}}^{i n t}+\overline{D_{R_{k}}^{i n t}} \gamma_{\mu} \mathcal{D}^{\mu} D_{R_{k}}^{i n t}\right\} \\
& i \sum_{k=1}^{3}\left\{\overline{\ell_{L_{k}}^{i n t}} \gamma_{\mu} \mathcal{D}^{\mu} \ell_{L_{k}}^{i n t}+\overline{\ell_{R_{k}}^{i n t}} \gamma_{\mu} \mathcal{D}^{\mu} \ell_{R_{k}}^{i n t}+\overline{\nu_{L_{k}}^{i n t}} \gamma_{\mu} \mathcal{D}^{\mu} \nu_{L_{k}}^{i n t}+\overline{\nu_{R_{k}}^{i n t}} \gamma_{\mu} \mathcal{D}^{\mu} \nu_{R_{k}}^{i n t}\right\}
\end{aligned}
$$

where $k$ runs over the three families and the greek indices $\mu, \nu$ run as customary on the space-time coordinates. The first two terms contain the gauge field tensors, similar to the electromagnetic tensor $F^{\mu \nu}$ :

$$
\begin{aligned}
\mathcal{B}_{\mu \nu} & =\partial_{\mu} \mathcal{B}_{\nu}-\partial_{\nu} \mathcal{B}_{\mu} \\
\mathcal{W}_{\mu \nu} & =\partial_{\mu} \mathbf{W}_{\nu}-\partial_{\nu} \mathbf{W}_{\mu}-i g\left(\mathbf{W}_{\mu} \mathbf{W}_{\nu}-\mathbf{W}_{\nu} \mathbf{W}_{\mu}\right)
\end{aligned}
$$

where $\mathbf{W}_{\mu}=\overrightarrow{\mathcal{W}}_{\mu} \cdot \vec{T} ; \vec{T}$ represents the weak isospin rotation matrices. The terms in curly brackets in (1.1) describe both the free motion and the interaction through the weak and electromagnetic currents. The covariant derivative $\mathcal{D}^{\mu}$ is:

$$
\mathcal{D}^{\mu}=\partial^{\mu}-i g T_{j} \mathcal{W}_{j}^{\mu}-i g^{\prime} \frac{Y}{2} \mathcal{B}^{\mu}
$$

where $g$ and $g^{\prime}$ are the coupling constants associated to the gauge fields $\mathcal{W}_{j}$ (weak isospin $S U(2)$ symmetry group) and $\mathcal{B}$ (hypercharge $U(1)$ symmetry group). When $i g T_{j} \mathcal{W}_{j}^{\mu}$ acts on the weak isospin doublets $T_{j}=\tau_{j} / 2$ where $\vec{\tau}$ are the Pauli matrices, otherwise $T_{j}=0$.

The Lagrangian in (1.1) contains massless fermions and bosons. Of course these particles have mass, but the gauge symmetry forbids adding mass terms $\propto B^{\mu} B_{\mu}$ to the Lagrangian, since they would not be gauge invariant. Concerning fermion masses, it is not possible to write a mass term that couples a $S U(2)_{I}$ doublet with a singlet with no external fields. The mechanism that allows one to introduce the mass terms for fermions and bosons is the well-known Higgs mechanism. It consists in adding to the Lagrangian (1.1) a new field with a specially crafted potential: the Higgs field, an isospin doublet of complex scalar fields with hypercharge $Y=1$ :

$$
\Phi=\left(\begin{array}{c}
\phi_{+} \\
\phi_{0}
\end{array}\right)
$$


The Higgs field Lagrangian is invariant under the $S U(2)_{I} \otimes U(1)_{Y}$ group:

$$
\mathcal{L}_{H}=\left(\mathcal{D}_{\mu} \Phi\right)^{\dagger}\left(\mathcal{D}^{\mu} \Phi\right)-\mu^{2}\left(\Phi^{\dagger} \Phi\right)-\lambda\left(\Phi^{\dagger} \Phi\right)^{2}
$$

where the derivative is given by Eq. (1.4). Analyzing the potential $V(\Phi)=\mu^{2}\left(\Phi^{\dagger} \Phi\right)+$ $\lambda\left(\Phi^{\dagger} \Phi\right)^{2}$ we find that for $\mu^{2}<0$ and $\lambda>0, V(\Phi)$ it has a minimum at $|\Phi|=\frac{-\mu^{2}}{2 \lambda}$. Therefore the expectation value on vacuum is not zero for at least one component of $\Phi$, and the fundamental state is not symmetric under the Lagrangian symmetry transformations. This phenomenon is called a spontaneously symmetry breaking and has many applications also outside the realm of particle physics. The application of the covariant derivatives on the fundamental Higgs field (the kinetic part of Eq. (1.6)) produces a gauge boson mass matrix that is not diagonal, implying that the gauge bosons mix into each other. The physical states responsible for the weak and electromagnetic interactions $\left(W^{+}, W^{-}, Z^{0}, \gamma\right)$ are the eigenstates obtained diagonalizing the mass matrix. The $Z^{0}$ boson and the photon are related to the $\mathcal{W}_{3}$ and $\mathcal{B}$ components of the gauge fields by a rotation of the Weinberg angle:

$$
\left(\begin{array}{c}
Z^{\mu} \\
A^{\mu}
\end{array}\right)=\left(\begin{array}{cc}
\cos \theta_{W} & -\sin \theta_{W} \\
\sin \theta_{W} & \cos \theta_{W}
\end{array}\right)\left(\begin{array}{c}
\mathcal{W}_{3}^{\mu} \\
\mathcal{B}^{\mu}
\end{array}\right)
$$

where $Z^{\mu}$ is the field associated with the $Z^{0}$ boson, $A^{\mu}$ is the field associated with the photon, and $\theta_{W}$ is Weinberg angle, $\sin ^{2} \theta_{W}=0.23122 \pm 0.00015$ [23]. The charged bosons $W^{+}$and $W^{-}$are the two orthogonal combinations of $W_{1}$ and $W_{2}$ :

$$
\begin{aligned}
& W_{+}^{\mu}=\frac{\mathcal{W}_{1}^{\mu}-i \mathcal{W}_{2}^{\mu}}{2} \\
& W_{-}^{\mu}=\frac{W_{1}^{\mu}+i W_{2}^{\mu}}{2}
\end{aligned}
$$

The coupling constants satisfy the following relations with the elementary electric charge $e^{1}$ :

$$
e=g \sin \theta_{W}=g^{\prime} \cos \theta_{W}
$$

Thanks to the spontaneously broken symmetry, the group $S U(2)_{I} \otimes U(1)_{Y}$ reduces to the group $U(1)_{Q}$ and the bosons of the weak interactions acquire a mass, while the photon does not.

The Lagrangian $(1.1)$ can be rewritten separating the free motion term $\left(\mathcal{L}_{0}\right)$ from the interaction part $\left(\mathcal{L}_{I}\right)$ :

$$
\mathcal{L}_{E W}=\mathcal{L}_{0}+\mathcal{L}_{I}
$$

\footnotetext{
${ }^{1}$ In the following we use natural units: $\hbar=1, e=1, c=1$.
} 
In particular, the interaction part can be written in terms of the coupling between the physical bosons and the fermion currents, divided in charged $\left(\mathcal{L}_{C C}\right)$ and neutral $\left(\mathcal{L}_{N C}\right)$ currents. The charged current acts inside the doublets and involves only the left hand components:

$$
J_{\mu}^{+}=i \sum_{k=1}^{3}\left\{\overline{U_{L_{k}}^{i n t}} \gamma_{\mu} D_{L_{k}}^{i n t}+\overline{\nu_{L_{k}}^{i n t}} \gamma_{\mu} \ell_{L_{k}}^{i n t}\right\}
$$

where $k$ runs over the three families.

The charged current Lagrangian becomes:

$$
\mathcal{L}_{C C}=\frac{g}{\sqrt{2}}\left(J_{\mu}^{+} W_{-}^{\mu}+\text { h.c. }\right) .
$$

This corresponds to the charged $\mathrm{V}-\mathrm{A}$ interaction.

The neutral current couples the two particles with the same chirality, both left or both right handed, therefore the index $L$ or $R$ is omitted in the following. The neutral current Lagrangian is:

$$
\mathcal{L}_{N C}=e J_{\mu}^{e m} A^{\mu}+\frac{g}{2 \cos \theta_{W}} J_{\mu}^{0} Z^{\mu}
$$

The electromagnetic current is:

$$
J_{\mu}^{e m}=i \sum_{k=1}^{3}\left\{q_{u}\left[\overline{U_{k}^{i n t}} \gamma_{\mu} U_{k}^{i n t}\right]+q_{d}\left[\overline{D_{k}^{i n t}} \gamma_{\mu} D_{k}^{i n t}\right]+\left[\overline{\ell_{k}^{i n t}} \gamma_{\mu} \ell_{k}^{i n t}\right]\right\}
$$

where $k$ runs over the three families, $q_{u}=+\frac{2}{3}$ and $q_{d}=-\frac{1}{3}$ are the electric charges of the quark up and down, respectively, in units of the positron charge. The neutral weak current is:

$$
\begin{gathered}
J_{\mu}^{0}=i \sum_{k=1}^{3}\left[\overline{U_{k}^{i n t}}\left(c_{V}^{k}-c_{A}^{k} \gamma^{5}\right) \gamma_{\mu} U_{k}^{i n t}+\overline{D_{k}^{i n t}}\left(c_{V}^{k}-c_{A}^{k} \gamma^{5}\right) \gamma_{\mu} D_{k}^{i n t}+\right. \\
\left.\overline{\ell_{k}^{i n t}}\left(c_{V}^{k}-c_{A}^{k} \gamma^{5}\right) \gamma_{\mu} \ell_{k}^{i n t}+\overline{\nu_{\ell_{k}}^{i n t}}\left(1-\gamma^{5}\right) \gamma_{\mu} \nu_{\ell_{k}}^{i n t}\right]
\end{gathered}
$$

where $k$ runs over the three families. The coefficients $c_{V}$ and $c_{A}$ represent the vector and pseudoscalar couplings for the fermions:

$$
\begin{aligned}
c_{V}^{f} & =I_{3}^{f}-2 Q_{f} \sin ^{2} \theta_{W} \\
c_{A}^{f} & =I_{3}^{f},
\end{aligned}
$$

where $I_{3}^{f}$ is the weak isospin of the fermion, $Q_{f}$ is its charge in units of the positron charge and $\theta_{W}$ is the Weinberg angle.

The fermion masses are generated by a Yukawa coupling between the Higgs field and the 
fermionic fields. For the quarks we have

$$
\mathcal{L}_{Y}=-\sum_{i, j=1}^{3}\left(Y_{i j}^{d} \overline{Q_{L, i}^{\mathrm{int}}} \phi d_{R, j}+Y_{i j}^{u} \overline{Q_{L, i}^{\mathrm{int}}} \phi^{*} \epsilon u_{R, j}+\text { h.c. }\right)
$$

where $i$ and $j$ run over the three families, $Y_{i j}^{u, d}$ are $3 \times 3$ complex matrices, and $\epsilon$ is the $2 \times 2$ antisymmetric tensor in the weak isospin space. When we substitute the Higgs field vacuum expectation value and diagonalize $Y_{i j}^{u, d}$ by 4 unitary matrices $V_{L, R}^{u, d}$,

$$
Y^{u, d} \rightarrow V_{L}^{u, d} Y^{u, d}\left(V_{R}^{u, d}\right)^{\dagger}
$$

we obtain the mass terms for the quarks. A similar procedure applies also to leptons, but it is not relevant for this work.

The diagonalization of $Y^{u, d}$ means that the interaction eigenstates $U^{i n t}$ and $D^{\text {int }}$ are not eigenstates of the free Lagrangian $(U$ and $D)$. This is obvious if we consider that the weak interaction changes the flavor of the quarks: the interaction cannot commute with the free Hamiltonian. We can look at this from a different point of view. Since the quark mass eigenstates differ from the interaction eigenstates we find four unitary matrices $\left(V_{L, R}^{u, d}\right)$ that allow us to change base and write the free Lagrangian eigenstates in terms of the interaction eigenstates:

$$
\begin{array}{rlrl}
D_{L_{i}} & =\left(V_{L}^{d}\right)_{i j} D_{L_{j}}^{i n t} \quad ; \quad D_{R_{i}}=\left(V_{R}^{d}\right)_{i j} D_{R_{j}}^{i n t} \\
U_{L_{i}} & =\left(V_{L}^{u}\right)_{i j} U_{L_{j}}^{\text {int }} \quad ; & U_{R_{i}}=\left(V_{R}^{u}\right)_{i j} U_{R_{j}}^{i n t}
\end{array}
$$

where the $V_{L, R}^{u, d}$ matrices are the same as in (1.17). The change of base has direct consequences on the charged currents (1.11), but not on the neutral ones (1.14), (1.15). Let's consider, as an example, the electromagnetic current of the up quark:

$$
J_{\mu}^{e m, u}=i q_{u} \overline{u^{i n t}} \gamma_{\mu} u^{i n t}
$$

and apply (1.18). We obtain:

$$
J_{\mu}^{e m, u}=i q_{u} \bar{u} \gamma_{\mu}\left(\sum_{k} V_{1 k}^{u} V_{k 1}^{u \dagger}\right) u
$$

where $V^{u}$ is unitary, thus we have $\sum_{k} V_{1 k}^{u} V_{k 1}^{u \dagger}=1$ and therefore the current is unchanged. The same applies to each term of the neutral current, hence the change of base has no effect on them. For the charged current part of the Lagrangian, on the other hand, the above argument does not apply. Let's consider the charged current involving the $W^{-}$ boson and the first quark generation:

$$
\mathcal{L}_{C C}=\frac{g}{\sqrt{2}} \bar{U}_{L_{i}} \gamma_{\mu}\left(V_{L_{i k}}^{u} V_{L_{k j}}^{d \dagger}\right) D_{L_{j}} W_{-}^{\mu} .
$$


The unitary matrix $V_{i j}=V_{L_{i k}}^{u} V_{L_{k j}}^{d \dagger}$ is known as the Cabibbo-Kobayashi-Maskawa (CKM) matrix and it represents the generalization of the Cabibbo mechanism from two to three generations.

\section{The Cabibbo-Kobayashi-Maskawa Matrix}

The CKM matrix connects the quark weak interaction eigenstates to the mass eigenstates. A $3 \times 3$ matrix is parameterizable, in general, with 18 parameters. The unitarity condition reduces the parameters to nine: three angles and six phases. Indeed, five of these phases can be absorbed in the wave function definitions of the quarks, therefore we are left with three angles and one phase. $C P$ Violation in the SM is due to this irreducible complex phase in the Lagrangian, since each term of the Lagrangian is transformed into its hermitian conjugate under $C P$, and therefore, if the Lagrangian contains complex terms, it will not be invariant under $C P$.

The CKM matrix can be written as:

$$
V_{C K M}=\left(\begin{array}{ccc}
V_{u d} & V_{u s} & V_{u b} \\
V_{c d} & V_{c s} & V_{c b} \\
V_{t d} & V_{t s} & V_{t b}
\end{array}\right)
$$

where $V_{q_{1} q_{2}}$ is the term relative to the transition $q_{1} \rightarrow q_{2}$. Many parameterizations are reported in literature, but two are the most commonly used: the standard parameterization, based on Euler angles, and the Wolfenstein parameterization, underlining the different orders of magnitude of the parameters.

The standard parameterization [23] is:

$$
V_{C K M}=\left(\begin{array}{ccc}
c_{12} c_{13} & s_{12} s_{13} & s_{13} e^{-i \delta} \\
-s_{12} c_{23}-c_{12} s_{23} s_{13} e^{i \delta} & c_{12} c_{23}-s_{12} s_{23} s_{13} e^{i \delta} & s_{23} c_{13} \\
s_{12} s_{23}-c_{12} c_{23} c_{13} e^{i \delta} & -c_{12} s_{23}-s_{12} c_{23} s_{13} e^{i \delta} & c_{23} c_{13}
\end{array}\right)
$$

where $c_{i j}=\cos \left(\theta_{i j}\right)$ and $s_{i j}=\sin \left(\theta_{i j}\right), \theta_{i j}$ are three real angles that can be chosen in the first quadrant; $\delta$ is the $C P$ violating phase.

Since experimentally $s_{13} \ll s_{23} \ll s_{12} \ll 1$, Wolfenstein [24] has defined a parameterization useful to underline this hierarchy. The four independent parameters used are $A, \lambda, \rho$ and $\eta$, defined in the following way:

$$
s_{12}=\lambda=\frac{\left|V_{u s}\right|}{\sqrt{\left|V_{u d}\right|^{2}+\left|V_{u s}\right|^{2}}} \quad, \quad s_{23}=A \lambda^{2}=\lambda \frac{\left|V_{c b}\right|}{\left|V_{u s}\right|} \quad, \quad s_{13} e^{i \delta}=A \lambda^{3}(\rho+i \eta)=V_{u b}^{*} .
$$

All four parameters are of order 1 with $\lambda=0.2253 \pm 0.0007$ [23] representing the small parameter (the sine of the Cabibbo angle) in which the CKM matrix can be expanded. 
The CKM matrix becomes:

$$
V_{C K M}=\left(\begin{array}{ccc}
1-\lambda^{2} / 2 & \lambda & A \lambda^{3}(\rho-i \eta) \\
-\lambda & 1-\lambda^{2} / 2 & A \lambda^{2} \\
A \lambda^{3}(1-\rho-i \eta) & -A \lambda^{2} & 1
\end{array}\right)+\mathcal{O}\left(\lambda^{4}\right) .
$$

Instead of $\rho$ and $\eta$, one often uses $\bar{\rho}$ and $\bar{\eta}$, defined by the complex relation:

$$
\bar{\rho}+i \bar{\eta}=-\frac{V_{u d} V_{u b}^{*}}{V_{c d} V_{c b}^{*}}
$$

independent of the CKM phase. The measured values of the other CKM parameters are:

$$
A=0.808_{-0.015}^{+0.022}, \quad \bar{\rho}=0.132_{-0.011}^{+0.022}, \quad \bar{\eta}=0.341 \pm 0.013 .
$$

The parameter $\eta$ is directly related to the $C P$ violating phase $\delta, \eta / \rho=\tan \delta$. Using this parameterization it is apparent that the elements on the diagonal are $\mathcal{O}(1)$ while, their values decrease rapidly away from the diagonal.

\section{The Unitary Triangles}

The unitary condition of the CKM matrix,

$$
V_{C K M}^{\dagger} V_{C K M}=V_{C K M} V_{C K M}^{\dagger}=\mathbf{1}
$$

allows to write relations among its elements. Let's consider the six relations corresponding to the off-diagonal terms of the identity matrix, the first three are from $V_{C K M}^{\dagger} V_{C K M}$, the second from $V_{C K M} V_{C K M}^{\dagger}$ :

$$
\begin{aligned}
& V_{u d} V_{u s}^{*}+V_{c d} V_{c s}^{*}+V_{t d} V_{t s}^{*}=0 \\
& V_{u d} V_{u b}^{*}+V_{c d} V_{c b}^{*}+V_{t d} V_{t b}^{*}=0 \\
& V_{u s} V_{u b}^{*}+V_{c s} V_{c b}^{*}+V_{t s} V_{t b}^{*}=0 \\
& V_{u d} V_{t d}^{*}+V_{u s} V_{t s}^{*}+V_{u b} V_{t b}^{*}=0 \\
& V_{u d} V_{c d}^{*}+V_{u s} V_{c s}^{*}+V_{u b} V_{c b}^{*}=0 \\
& V_{c d} V_{t d}^{*}+V_{c s} V_{t s}^{*}+V_{c b} V_{t b}^{*}=0 .
\end{aligned}
$$

These relations can be represented on a complex plane as triangles, called Unitary Triangles (UT). All triangles have the same area, which represents a phase-convention-independent measurement of $C P$ violation. In Appendix A we demonstrate that all triangles have the same area and we compute it as invariant.

Depending on the length of the sides of each triangle, they are more or less degenerate. The least degenerate triangles are the ones in (1.25) and (1.27), since each side is $\mathcal{O}\left(\lambda^{3}\right)$. 
Let's consider the one in (1.25) and divide the equation by $V_{c d} V_{c b}^{*}$,

$$
\frac{V_{u d} V_{u b}^{*}}{V_{c d} V_{c b}^{*}}+1+\frac{V_{t d} V_{t b}^{*}}{V_{c d} V_{c b}^{*}}=0
$$

We represent this triangle in the complex plane $(\bar{\rho}, \bar{\eta})$ in Fig. 1.1.

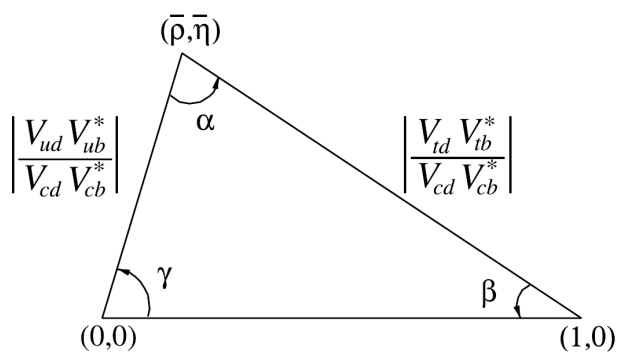

Figure 1.1: Unitary Triangle of Eq. (1.25) in the $(\bar{\rho}, \bar{\eta})$ plane.

Tests of the CKM paradigm consist of over-constraining the free vertex by measuring the angles of the triangle,

$$
\alpha=\arg \left[\frac{V_{u b}^{*} V_{u d}}{V_{t b}^{*} V_{t d}}\right] \quad, \quad \beta=\arg \left[\frac{V_{t b}^{*} V_{t d}}{V_{c b}^{*} V_{c d}}\right] \quad, \quad \gamma=\arg \left[\frac{V_{c b}^{*} V_{c d}}{V_{u b}^{*} V_{u d}}\right] .
$$

and the sides. In Fig. 1.2 we report the current experimental situation of the measurements of the Unitary Triangle and their combination computed by the CKMFitter Group [25].

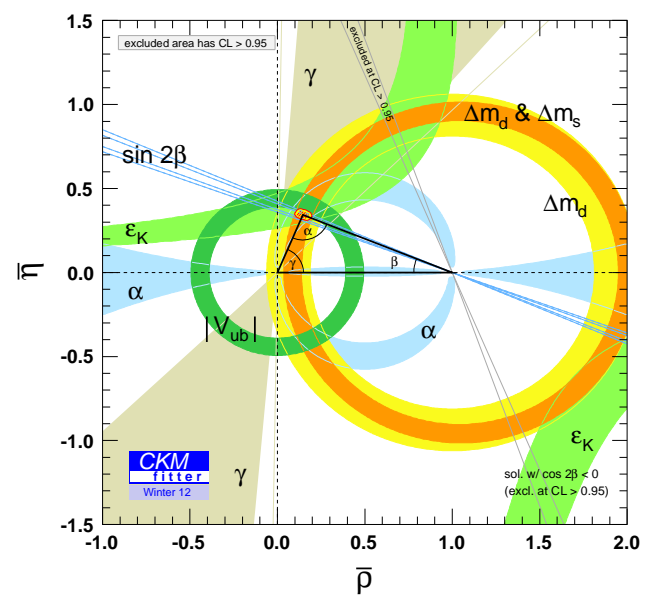

Figure 1.2: Measurements of the Unitary Triangle in the $(\bar{\rho}, \bar{\eta})$ plane and their combination computed by the CKMFitter Group (updated results and plots available at: http://ckmfitter.in2p3.fr). 
The triangle relative to charm physics is the one in (1.28). It is very degenerate since two of the sides are $\mathcal{O}(\lambda)$ and the third is $\mathcal{O}\left(\lambda^{5}\right)$. Although the area is the same as the other triangles, performing measurements on this one is more than a challenge.

\section{$1.2 \quad$ Neutral Meson Mixing}

In the SM there are four meson-antimeson mixing systems: $K^{0}-\bar{K}^{0}, B^{0}-\bar{B}^{0}, B_{s}-\bar{B}_{s}$ and $D^{0}-\bar{D}^{0}$. These mesons are produced in flavor eigenstates that are not eigenstates of the free Hamiltonian and therefore do not evolve as free particle, but mix one into the other with their time evolution and decay governed by the weak interaction. They can be produced as meson-antimeson pairs by transitions involving the strong interaction, or as single mesons in weak decays of a heavier particle.

In Sec. 1.2.1 we present their time evolution, and in Sec. 1.2.2 we present a modelindependent classification of the $C P$ violation effects in this type of systems. In Sec. 1.2.3 we briefly discuss the differences among the 4 systems, and in particular the peculiarities of the $D^{0}-\bar{D}^{0}$ system.

\subsubsection{Time Evolution of the Flavor Eigenstates}

Let us consider a state composed by a neutral meson $P^{0}$ and its own antiparticle $\bar{P}^{0}$, distinguished only by the flavor $F$, an internal quantum number. The state at $t=0$

$$
|\Psi(0)\rangle=a(0)\left|P^{0}\right\rangle+b(0)\left|\bar{P}^{0}\right\rangle
$$

will evolve following Schrödinger equation,

$$
i \frac{\partial}{\partial t}|\Psi(t)\rangle=\mathbf{H}|\Psi(t)\rangle
$$

where $\mathbf{H}$ is the Hamiltonian governing the system. At a certain time $t$ we can write

$$
|\Psi(t)\rangle=a(t)\left|P^{0}\right\rangle+b(t)\left|\bar{P}^{0}\right\rangle+\sum_{k} c_{k}(t)\left|f_{k}\right\rangle
$$

where $k$ runs over all the possible final states $\left|f_{k}\right\rangle$ in which $P^{0}$ or $\bar{P}^{0}$ can decay.

In order to describe mixing, observing Eq. (1.31), we restrict ourselves to the subspace relative to $\left|P^{0}\right\rangle$ and $\left|\bar{P}^{0}\right\rangle$ introducing an effective Hamiltonian $\mathcal{H}_{\text {eff }}$, no longer hermitian. The Schrödinger equation can be re-written as:

$$
i \frac{\partial}{\partial t}\left(\begin{array}{l}
a(t) \\
b(t)
\end{array}\right)=\left(\begin{array}{ll}
\mathcal{H}_{11} & \mathcal{H}_{12} \\
\mathcal{H}_{21} & \mathcal{H}_{22}
\end{array}\right)\left(\begin{array}{l}
a(t) \\
b(t)
\end{array}\right) .
$$

The effective Hamiltonian is a $2 \times 2$ complex matrix that can be parameterized with two 
hermitian matrices: $\mathbf{M}$, the mass matrix and $\boldsymbol{\Gamma}$, the decay matrix:

$$
\mathcal{H}_{\mathrm{eff}}=\mathbf{M}-\frac{i}{2} \boldsymbol{\Gamma}
$$

with $\mathbf{M}_{i j}=\mathbf{M}_{j i}^{*}$ and $\boldsymbol{\Gamma}_{i j}=\boldsymbol{\Gamma}_{j i}^{*}$. The decay rate corresponds to the expectation value of $\boldsymbol{\Gamma}$ on the state $\Psi(t)$ :

$$
\frac{d}{d t}\langle\Psi(t) \mid \Psi(t)\rangle=-\langle\Psi(t)|\Gamma| \Psi(t)\rangle
$$

The matrix $\mathbf{M}-\frac{i}{2} \boldsymbol{\Gamma}$ has 8 free parameters, but, if the effective Hamiltonian benefits from some symmetries the number of degrees of freedom decreases. In Tab. 1.1 we report the consequences of the discrete symmetries $C, P, T$ and their combinations on the elements of the effective Hamiltonian [26].

\begin{tabular}{rll}
\hline \hline$C P T$ invariance & $\Rightarrow$ & $M_{11}=M_{22} ; \Gamma_{11}=\Gamma_{22}$ \\
$C P$ invariance & $\Rightarrow$ & $M_{11}=M_{22} ; \Gamma_{11}=\Gamma_{22} ; \Im\left[\Gamma_{12} / M_{12}\right]=0$ \\
$T$ invariance & $\Rightarrow \Im\left[\Gamma_{12} / M_{12}\right]=0$ \\
\hline \hline
\end{tabular}

Table 1.1: Relations among the elements of $\mathbf{M}$ and $\boldsymbol{\Gamma}$ as consequences of $C P T, C P$ and $T$ invariances.

From now on we assume the $C P T$ invariance, in other words $M_{11}=M_{22}$ and $\Gamma_{11}=\Gamma_{22}$. Let us call the $\mathcal{H}_{\text {eff }}$ eigenstates $\left|P_{1}\right\rangle$ and $\left|P_{2}\right\rangle$ :

$$
\begin{aligned}
& \left|P_{1}\right\rangle=p\left|P^{0}\right\rangle+q\left|\bar{P}^{0}\right\rangle \\
& \left|P_{2}\right\rangle=p\left|P^{0}\right\rangle-q\left|\bar{P}^{0}\right\rangle
\end{aligned}
$$

with $|p|^{2}+|q|^{2}=1$, with eigenvalues:

$$
\begin{aligned}
\mathcal{H}_{\mathrm{eff}}\left|P_{1,2}\right\rangle & =\lambda_{1,2}\left|P_{1,2}\right\rangle \\
\lambda_{1} & \equiv M_{1}-\frac{i}{2} \Gamma_{1}=\mathbf{M}_{11}-\frac{i}{2} \boldsymbol{\Gamma}_{11}+\frac{q}{p}\left(\mathbf{M}_{12}-\frac{i}{2} \boldsymbol{\Gamma}_{12}\right) \\
\lambda_{2} & \equiv M_{2}-\frac{i}{2} \Gamma_{2}=\mathbf{M}_{11}-\frac{i}{2} \boldsymbol{\Gamma}_{11}-\frac{q}{p}\left(\mathbf{M}_{12}-\frac{i}{2} \boldsymbol{\Gamma}_{12}\right) .
\end{aligned}
$$

The complex number $q / p$, written in terms of $\mathbf{M}$ and $\boldsymbol{\Gamma}$ elements is:

$$
\frac{q}{p}= \pm \sqrt{\frac{M_{12}^{*}-\frac{i}{2} \Gamma_{12}^{*}}{M_{12}-\frac{i}{2} \Gamma_{12}}}
$$

We choose the plus sign; choosing the minus is just equivalent to exchanging $\left|P_{1}\right\rangle$ with $\left|P_{2}\right\rangle$. We define two important quantities: $\Delta M$, the difference of masses of $\left|P_{1}\right\rangle$ and $\left|P_{2}\right\rangle$ 
and $\Delta \Gamma$, the difference of the decay widths:

$$
\begin{aligned}
\Delta M & \equiv M_{2}-M_{1}=-2 \Re\left[\frac{q}{p}\left(M_{12}-\frac{i}{2} \Gamma_{12}\right)\right] \\
\Delta \Gamma & \equiv \Gamma_{1}-\Gamma_{2}=4 \Im\left[\frac{q}{p}\left(M_{12}-\frac{i}{2} \Gamma_{12}\right)\right],
\end{aligned}
$$

and define the average width:

$$
\Gamma=\frac{\Gamma_{1}+\Gamma_{2}}{2}
$$

The time evolution of the $\mathcal{H}_{\text {eff }}$ eigenstates is straightforward:

$$
\begin{aligned}
\left|P_{1}(t)\right\rangle & =e^{-i \lambda_{1} t}\left|P_{1}\right\rangle \\
\left|P_{2}(t)\right\rangle & =e^{-i \lambda_{2} t}\left|P_{2}\right\rangle
\end{aligned}
$$

and it can be easily shown that the probability of changing state is zero while the probability of remaining in the same state decreases exponentially with lifetime $1 / \Gamma_{1,2}$ :

$$
\begin{aligned}
& \operatorname{Prob}\left(P_{1} \rightarrow P_{2}, t\right) \quad \propto \quad\left|\left\langle P_{2}(t) \mid P_{1}\right\rangle\right|^{2}=0 \\
& \operatorname{Prob}\left(P_{1} \rightarrow P_{1}, t\right) \quad \propto \quad\left|\left\langle P_{1}(t) \mid P_{1}\right\rangle\right|^{2} \propto e^{-\Gamma_{1} t} .
\end{aligned}
$$

The description of the time evolution of the states with defined flavor, $\left|P^{0}\right\rangle$ and $\left|\bar{P}^{0}\right\rangle$, is slightly more complicated and requires some more math.

Let us call $\left|P^{0}(t)\right\rangle$ the state of a meson that was a $\left|P^{0}\right\rangle$ at $t=0\left(\left|P^{0}\right\rangle=\frac{1}{2 p}\left[\left|P_{1}\right\rangle+\left|P_{2}\right\rangle\right]\right)$ and let us write it as a superposition of $\left|P_{1}(t)\right\rangle$ and $\left|P_{2}(t)\right\rangle$, with explicit time dependence:

$$
\left|P^{0}(t)\right\rangle=\frac{1}{2 p}\left[\left|P_{1}(t)\right\rangle+\left|P_{2}(t)\right\rangle\right]=\frac{1}{2 p}\left[e^{-i \lambda_{1} t}\left|P_{1}\right\rangle+e^{-i \lambda_{2} t}\left|P_{2}\right\rangle\right] .
$$

If we rewrite $\left|P_{1}\right\rangle$ and $\left|P_{2}\right\rangle$ in terms of $\left|P^{0}\right\rangle$ and $\left|\bar{P}^{0}\right\rangle$ using Eq. (1.32), we obtain:

$$
\left|P^{0}(t)\right\rangle=g_{+}(t)\left|P^{0}\right\rangle+\frac{q}{p} g_{-}(t)\left|\bar{P}^{0}\right\rangle
$$

where we have incorporated the time dependence in two functions $g_{+}(t)$ and $g_{-}(t)$, defined as:

$$
g_{ \pm}(t)=\frac{e^{-i \lambda_{1} t} \pm e^{-i \lambda_{2} t}}{2}
$$

In the same way we can write the time evolution of the meson that was a $\left|\bar{P}^{0}\right\rangle$ at time $t=0\left(\left|\bar{P}^{0}\right\rangle=\frac{1}{2 q}\left[\left|P_{1}\right\rangle-\left|P_{2}\right\rangle\right]\right)$ :

$$
\left|\bar{P}^{0}(t)\right\rangle=g_{+}(t)\left|\bar{P}^{0}\right\rangle+\frac{p}{q} g_{-}(t)\left|P^{0}\right\rangle
$$


We can compute the probability that a meson created with a well-defined flavor content at time $t=0$, has the opposite flavor at a later time $t>0$ as a consequence of the $\Delta F=2$ transitions mediated by weak interaction. The probability that the meson does not change flavor is the same for $\left|P^{0}\right\rangle$ and $\left|\bar{P}^{0}\right\rangle$ :

$$
\begin{aligned}
& \operatorname{Prob}\left(P^{0} \rightarrow P^{0}, t\right) \quad \propto \quad\left|\left\langle P^{0}(t) \mid P^{0}\right\rangle\right|^{2}=\left|g_{+}(t)\right|^{2} \\
& \operatorname{Prob}\left(\bar{P}^{0} \rightarrow \bar{P}^{0}, t\right) \quad \propto \quad\left|\left\langle\bar{P}^{0}(t) \mid \bar{P}^{0}\right\rangle\right|^{2}=\left|g_{+}(t)\right|^{2}
\end{aligned}
$$

while the probability of a flavor change is different for the two mesons if $|q / p| \neq 1$ :

$$
\begin{aligned}
& \operatorname{Prob}\left(P^{0} \rightarrow \bar{P}^{0}, t\right) \propto\left|\left\langle P^{0}(t) \mid \bar{P}^{0}\right\rangle\right|^{2}=\left|\frac{q}{p}\right| \cdot\left|g_{-}(t)\right|^{2} \\
& \operatorname{Prob}\left(\bar{P}^{0} \rightarrow P^{0}, t\right) \propto\left|\left\langle\bar{P}^{0}(t) \mid P^{0}\right\rangle\right|^{2}=\left|\frac{p}{q}\right| \cdot\left|g_{-}(t)\right|^{2} .
\end{aligned}
$$

Setting $|q / p|=1$, Eqs. (1.41) and (1.43) can be rewritten as

$$
\begin{aligned}
& \operatorname{Prob}\left(P^{0} \rightarrow P^{0}, t\right) \propto e^{-\Gamma t}[\cosh (2 \Delta \Gamma t)+\cos (\Delta M t] \\
& \operatorname{Prob}\left(P^{0} \rightarrow \bar{P}^{0}, t\right) \propto e^{-\Gamma t}[\cosh (2 \Delta \Gamma t)-\cos (\Delta M t]
\end{aligned}
$$

In Fig. 1.3 we show the probabilities of changing (Eq. (1.46)) and not changing (Eq. (1.45)) the flavor for different values for $\Delta M$ and $\Delta \Gamma$. These are examples that do not correspond to any of the four mixing systems in the SM. The presence of mixing alters the exponential

$\Delta M=5 \Gamma, \Delta \Gamma=0:$

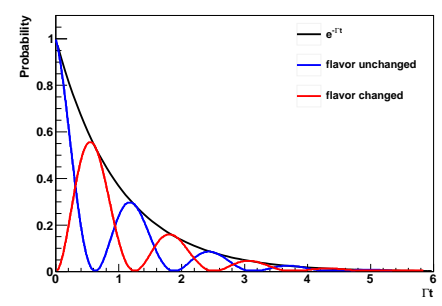

$\Delta M=0, \Delta \Gamma=1.6 \Gamma:$

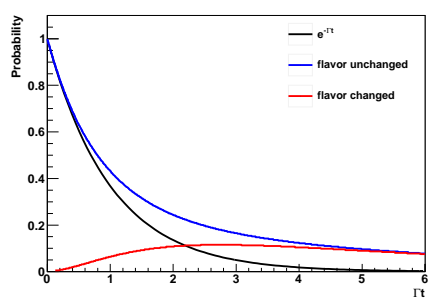

$\Delta M=5 \Gamma, \Delta \Gamma=1.6 \Gamma:$

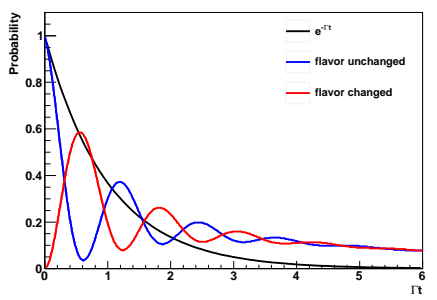

Figure 1.3: Probability of changing the flavor (red line), non changing it (blue) and the $e^{-\Gamma t}$ function (black) for three different sets of $(\Delta M, \Delta \Gamma):(5 \Gamma, 0)$ for the left plot, $(0,1.6 \Gamma)$ for the center plot and $(5 \Gamma, 1.6 \Gamma)$ for the right plot.

distribution for the $P^{0}$ decay into a final state $f$. The oscillation period is proportional to the inverse of $\Delta M$.

Let's now complete the time evolution picture computing the decay probability in a final state $f$, different from $\left|P^{0}\right\rangle$ or $\left|\bar{P}^{0}\right\rangle$.

Let us suppose that the weak interaction (described by $H_{w}$ ) is responsible for the transitions with $\Delta F \neq 0$ (i.e. in the decays but also in the mixing) and that other interactions (described by $\left.H_{0}\right)$ can't change the flavor. We indicate with $A_{f}\left(\bar{A}_{f}\right)$ the $P^{0}\left(\bar{P}^{0}\right)$ decay 
amplitude into the final state $f$ :

$$
\begin{aligned}
& A_{f}=\left\langle f\left|H_{w}\right| P^{0}\right\rangle \\
& \bar{A}_{f}=\left\langle f\left|H_{w}\right| \bar{P}^{0}\right\rangle
\end{aligned}
$$

and define the parameter $\lambda_{f}$ as

$$
\lambda_{f} \equiv \frac{q}{p} \frac{\bar{A}_{f}}{A_{f}}
$$

The partial decay width for the decays $P^{0} \rightarrow f$ is proportional to the square of the amplitude:

$$
\begin{aligned}
\Gamma\left(P^{0}(t) \rightarrow f\right) & \propto\left|\left\langle f\left|H_{w}\right| P^{0}(t)\right\rangle\right|^{2}= \\
& =\left|g_{+}(t)\left\langle f\left|H_{w}\right| P^{0}\right\rangle+\frac{q}{p} g_{-}(t)\left\langle f\left|H_{w}\right| \bar{P}^{0}\right\rangle\right|^{2}= \\
& =\left|g_{+}(t) A_{f}+\frac{q}{p} g_{-}(t) \bar{A}_{f}\right|^{2}
\end{aligned}
$$

and similarly for $\bar{P}^{0}$ :

$$
\Gamma\left(\bar{P}^{0}(t) \rightarrow f\right) \propto\left|\left\langle f\left|H_{w}\right| \bar{P}^{0}(t)\right\rangle\right|^{2}=\left|g_{+}(t) \bar{A}_{f}+\frac{p}{q} g_{-}(t) A_{f}\right|^{2} .
$$

If we isolate the amplitude square and substitute the expressions of $g_{ \pm}(t)$ in terms of $\lambda_{f}$, $\Delta M$ and $\Delta \Gamma$, we find:

$$
\begin{aligned}
\Gamma\left(P^{0}(t) \rightarrow f\right) \propto \frac{1}{2}\left|A_{f}\right|^{2} e^{-\Gamma t} & {\left[\left(1+\left|\lambda_{f}\right|^{2}\right) \cosh \frac{\Delta \Gamma}{2} t+\left(1-\left|\lambda_{f}\right|^{2}\right) \cos \Delta M t\right.} \\
& \left.-2 \Re\left(\lambda_{f}\right) \sinh \frac{\Delta \Gamma}{2} t+2 \Im\left(\lambda_{f}\right) \sin \Delta M t\right] \\
\Gamma\left(\bar{P}^{0}(t) \rightarrow f\right) \propto \frac{1}{2}\left|\bar{A}_{f}\right|^{2} e^{-\Gamma t} & {\left[\left(1+\left|\lambda_{f}^{-1}\right|^{2}\right) \cosh \frac{\Delta \Gamma}{2} t+\left(1-\left|\lambda_{f}^{-1}\right|^{2}\right) \cos \Delta M t\right.} \\
& \left.-2 \Re\left(\lambda_{f}^{-1}\right) \sinh \frac{\Delta \Gamma}{2} t+2 \Im\left(\lambda_{f}^{-1}\right) \sin \Delta M t\right]
\end{aligned}
$$

For completeness we also report the results for decays to the $C P$-conjugate final state $\bar{f}$ :

$$
\begin{aligned}
\Gamma\left(P^{0}(t) \rightarrow \bar{f}\right) \propto \frac{1}{2}\left|A_{\bar{f}}\right|^{2} e^{-\Gamma t} & {\left[\left(1+\left|\lambda_{\bar{f}}\right|^{2}\right) \cosh \frac{\Delta \Gamma}{2} t+\left(1-\left|\lambda_{\bar{f}}\right|^{2}\right) \cos \Delta M t\right.} \\
& \left.-2 \Re\left(\lambda_{\bar{f}}\right) \sinh \frac{\Delta \Gamma}{2} t+2 \Im\left(\lambda_{\bar{f}}\right) \sin \Delta M t\right] \\
\Gamma\left(\bar{P}^{0}(t) \rightarrow \bar{f}\right) \propto \frac{1}{2}\left|\bar{A}_{\bar{f}}\right|^{2} e^{-\Gamma t} & {\left[\left(1+\left|\lambda_{\bar{f}}^{-1}\right|^{2}\right) \cosh \frac{\Delta \Gamma}{2} t+\left(1-\left|\lambda_{\bar{f}}^{-1}\right|^{2}\right) \cos \Delta M t\right.} \\
& \left.-2 \Re\left(\lambda_{\bar{f}}^{-1}\right) \sinh \frac{\Delta \Gamma}{2} t+2 \Im\left(\lambda_{\bar{f}}^{-1}\right) \sin \Delta M t\right]
\end{aligned}
$$




\subsubsection{CP Violation}

As introduced in Sec. 1.1, CP violation is parameterized in the SM through the complex phase of the CKM matrix. In the following we present a model-independent classification of $C P$ violation mechanisms.

In a neutral meson system, $C P$ violation can occur in three ways: in the decay, in the mixing, or in the interference between decays with and without mixing. $C P$ violation in the decay is also possible for charged particles.

\section{$C P$ Violation in the Decay}

This type of $C P V$ occurs when the process $P \rightarrow f$ has a different probability of the $C P$ conjugate process $\bar{P} \rightarrow \bar{f}$. This implies that the ratio of the two amplitudes $A_{f}=\left\langle f\left|H_{w}\right| P\right\rangle$ and $\bar{A}_{\bar{f}}=\left\langle\bar{f}\left|H_{w}\right| \bar{P}\right\rangle$ is different from one in magnitude:

$$
\left|\frac{A_{f}}{\bar{A}_{\bar{f}}}\right| \neq 1 \text {. }
$$

The experimental observable is the asymmetry

$$
\mathcal{A}_{f}=\frac{\Gamma(\bar{P} \rightarrow \bar{f})-\Gamma(P \rightarrow f)}{\Gamma(\bar{P} \rightarrow \bar{f})+\Gamma(P \rightarrow f)}=\frac{\left|\bar{A}_{\bar{f}} / A_{f}\right|^{2}-1}{\left|\bar{A}_{\bar{f}} / A_{f}\right|^{2}+1}
$$

It is important to note that, in order to exhibit this type of $C P V$, it is necessary that the process $P \rightarrow f$ proceeds through at least two amplitudes with different phases:

$$
\begin{aligned}
& A_{f}=a_{1} e^{i\left(\delta_{1}+\phi_{1}\right)}+a_{2} e^{i\left(\delta_{2}+\phi_{2}\right)} \\
& \bar{A}_{\bar{f}}=a_{1} e^{i\left(\delta_{1}-\phi_{1}\right)}+a_{2} e^{i\left(\delta_{2}-\phi_{2}\right)},
\end{aligned}
$$

where $\delta_{i}$ are $C P$-invariant strong phases, and $\phi_{i}$ are the $C P$-violating weak phases, which change sign under $C P$. The sensitivity to this type of $C P V$ is present only when the two amplitudes have different strong and weak phases, i.e. $\delta_{1} \neq \delta_{2}$, and $\phi_{1} \neq \phi_{2}$, since

$$
\left|A_{f}\right|^{2}-\left|\bar{A}_{\bar{f}}\right|^{2}=a_{1}^{2} a_{2}^{2} \sin \left(\delta_{1}-\delta_{2}\right) \sin \left(\phi_{1}-\phi_{2}\right)
$$

This type of $C P V$ involves the presence of the uneliminable weak phase in the physical decay amplitudes $A_{f}$ and $\bar{A}_{\bar{f}}$. In this case we talk of direct $C P V$.

$C P V$ in the decay has been observed in neutral $K$ decays and its detection ruled out the superweak model proposed by Wolfenstein in order to explain the $C P$ violation observed in 1964. 


\section{$C P$ Violation in the Mixing}

As shown in Tab. 1.1, the invariance of the Hamiltonian under the discrete symmetry operators $C, P$ and $T$, or their combinations, introduces constraints among the elements of $\mathbf{M}$ and $\boldsymbol{\Gamma}$. If $C P$ is a good symmetry, $\Im\left[\Gamma_{12} / M_{12}\right]=0$, and $q / p$ is complex number with a magnitude of one, in other words, a phase:

$$
\frac{q}{p}=\sqrt{\frac{M_{12}^{*}}{M_{12}}} \sqrt{\frac{1-\frac{i}{2} \frac{\Gamma_{12}^{*}}{M_{12}^{*}}}{1-\frac{i}{2} \frac{\Gamma_{12}}{M_{12}}}}=\sqrt{\frac{M_{12}^{*}}{M_{12}}} \quad \Rightarrow \quad\left|\frac{q}{p}\right|=1 .
$$

The condition $|q / p| \neq 1$ constitutes a $C P$ or $T$ violation, and would imply that the probability of the transition $\left|P^{0}\right\rangle \rightarrow\left|\bar{P}^{0}\right\rangle$ is different from the reversed one $\left|\bar{P}^{0}\right\rangle \rightarrow\left|P^{0}\right\rangle$, as can be seen in Eqs. (1.43) and (1.44).

In order to directly measure this type of $C P V$, a possibility is to choose a leptonic or semileptonic decay channel which determines the flavor of the meson at decay time. Knowing the flavor of the meson at production we construct the asymmetry $\mathcal{A}_{S L}$ :

$$
\mathcal{A}_{S L}=\frac{\Gamma\left(\bar{P}^{0}(t) \rightarrow \ell^{+} X\right)-\Gamma\left(P^{0}(t) \rightarrow \ell^{-} X\right)}{\Gamma\left(\bar{P}^{0}(t) \rightarrow \ell^{+} X\right)+\Gamma\left(P^{0}(t) \rightarrow \ell^{-} X\right)}=\frac{1-|q / p|^{4}}{1+|q / p|^{4}} .
$$

This type of $C P V$ does not involve any $\Delta F=1$ decay amplitude, consequently any uneliminable phase in a decay amplitude, it is therefore an example of indirect $C P V$.

$C P V$ in mixing has also been observed in the $K^{0}-\bar{K}^{0}$ system [27] using the $K_{L}^{0} \rightarrow \pi e \nu_{e}$ decays.

\section{$C P$ Violation in the Interference between Decays with and without Mixing}

In case the final state is accessible to both $P^{0}$ and $\bar{P}^{0}$, there is a third possible type of $C P$ violation. Since starting from the same flavor at production, the final state can be reached in two possible ways:

1. through a direct decay: $P^{0} \rightarrow f$;

2. through mixing and then decay: $P^{0} \rightarrow \bar{P}^{0} \rightarrow f$

This type of $C P V$ occurs in the interference between these two paths and it is possible even in case $C P$ is conserved separately in the decay and in mixing.

We recall the definition of $\lambda_{f}$ :

$$
\lambda_{f} \equiv \frac{q}{p} \frac{\bar{A}_{f}}{A_{f}}=\left|\frac{q}{p} \frac{\bar{A}_{f}}{A_{f}}\right| e^{i\left(\delta_{f}+\phi_{f}\right)},
$$


where $\phi_{f}$ and $\delta_{f}$ are the weak and strong phases, respectively, and $A_{f}\left(\bar{A}_{f}\right)$ is the decay amplitude of $P^{0}\left(\bar{P}^{0}\right) \rightarrow f$. It is worth noticing that the phase of $\lambda_{f}$ is physical, independent of the choice of the relative phase between $P^{0}$ and $\bar{P}^{0}$.

$C P V$ of this type occurs when the weak phase of $\lambda_{f}$ is different from zero, $\phi_{f} \neq 0$.

Suppose there is no $C P V$ in the decay nor in mixing, then:

$$
\left|\frac{q}{p}\right|=1 \quad \text { and } \quad\left|\frac{\bar{A}_{\bar{f}}}{A_{f}}\right|=1 .
$$

In this case, any difference between $\Gamma\left(P^{0}(t) \rightarrow f\right)$ and $\Gamma\left(\bar{P}^{0}(t) \rightarrow \bar{f}\right)$ is an evidence of $C P V$ in the interference between decays with and without mixing. Looking the Eqs. (1.50) and (1.53), under the hypotheses in (1.61), the terms in which these difference can appear are the ones depending on the argument of $\lambda_{f}$ and $\lambda_{\bar{f}}^{-1}$. Suppose there is only one amplitude contributing to the decay $P^{0} \rightarrow f$, that we parameterize as:

$$
A_{f}=a e^{i\left(\Delta_{f}+\Phi_{f}\right)}
$$

where $\Delta_{f}$ is the $C P$ conserving strong phase and $\Phi_{f}$ the $C P$-violating one. If we consider the $C P$-conjugate process $\bar{P}^{0} \rightarrow \bar{f}$ we can parameterize its amplitude as:

$$
\bar{A}_{\bar{f}}=a e^{i\left(\Delta_{f}-\Phi_{f}\right)} .
$$

Similarly for the processes $P^{0} \rightarrow \bar{f}$ and $\bar{P}^{0} \rightarrow f$, we can write, respectively:

$$
A_{\bar{f}}=r a e^{i\left(\Delta_{\bar{f}}+\Phi_{\bar{f}}\right)} \quad \text { and } \quad \bar{A}_{f}=r a e^{i\left(\Delta_{\bar{f}}-\Phi_{\bar{f}}\right)}
$$

where $r=\left|A_{\bar{f}} / A_{f}\right|$ and $\Delta_{\bar{f}} \neq \Delta_{f}$ and $\Phi_{\bar{f}} \neq \Phi_{f}$ and the $C P$ conserving and $C P$ violating phases, respectively, of the $P^{0} \rightarrow \bar{f}$ decay.

We can now compute $\lambda_{f}$ and $\lambda_{\bar{f}}^{-1}$ :

$$
\begin{aligned}
\lambda_{f} & =\frac{q}{p} \frac{\bar{A}_{f}}{A_{f}}=r e^{i\left[\left(\Delta_{\bar{f}}-\Delta_{f}\right)-\left(\Phi_{\bar{f}}-\Phi_{f}\right)+\Phi_{M}\right]} \\
\lambda_{\bar{f}}^{-1} & =\frac{p}{q} \frac{A_{\bar{f}}}{\bar{A}_{\bar{f}}}=r e^{i\left[\left(\Delta_{\bar{f}}-\Delta_{f}\right)+\left(\Phi_{\bar{f}}-\Phi_{f}\right)-\Phi_{M}\right]},
\end{aligned}
$$

with $\Phi_{M}=\arg (q / p)$. Therefore, $\lambda_{f}$ and $\lambda_{\bar{f}}^{-1}$ have the same magnitudes but different arguments if their weak phase $\phi_{f}=\Phi_{\bar{f}}+\Phi_{f}+\Phi_{M}$ is different from zero.

The weak phase $\phi_{f}$ has two independent contributions: $\Phi_{M}$ from mixing, independent of the final state; and $\Phi_{\bar{f}}+\Phi_{f}$ from the amplitudes ratio, that depends on final state. Therefore this type of $C P V$ may have both direct and indirect $C P V$ contributions. 
In case the final state is a $C P$ eigenstate $\left(|\bar{f}\rangle=C P|f\rangle=\eta_{f}|f\rangle\right)$ then $\Delta_{f}=\Delta_{\bar{f}}$, hence $\delta_{f}=0$, and the signature for $C P V$ becomes $\Im\left(\lambda_{f}\right) \neq 0$. If the weak phase is different for two different final states $f_{1}$ and $f_{2}, \Im\left(\lambda_{f_{1}}\right) \neq \Im\left(\lambda_{f_{2}}\right)$, then there is a contribution of direct $C P V$.

From an experimental point of view, a possibility to detect this type of $C P V$ is measuring the integrated asymmetry in $C P$ eigenstates, $\mathcal{A}_{f_{C P}}$ :

$$
\begin{aligned}
\mathcal{A}_{f_{C P}} & =\frac{\Gamma\left(\bar{P}^{0}(t) \rightarrow f_{C P}\right)-\Gamma\left(P^{0}(t) \rightarrow f_{C P}\right)}{\Gamma\left(\bar{P}^{0}(t) \rightarrow f_{C P}\right)+\Gamma\left(P^{0}(t) \rightarrow f_{C P}\right)}= \\
& =\frac{\left(1-\left|\lambda_{f}\right|^{2}\right) \cos (\Delta M t)-2 \Im \lambda_{f} \sin (\Delta M t)}{1+\left|\lambda_{f}\right|^{2}} .
\end{aligned}
$$

This type of $C P V$ is the one observed BABAR and Belle in the $B^{0} \rightarrow J / \psi K_{S}^{0}$ channel.

\subsubsection{Mixing Phenomenology in the Standard Model}

There are two types of contributions to the mixing amplitudes: the short-distance and the long-distance contributions. The length scale is defined by comparing the space-time distance traveled by the intermediate states $I$ to the typical scale of the strong interactions. The SM diagrams for the $D^{0}-\bar{D}^{0}$ mixing are reported in Fig. 1.4.
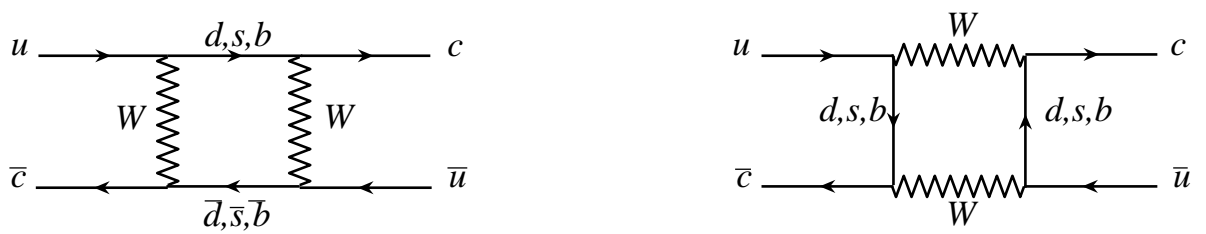

Figure 1.4: SM diagrams for the $D^{0}-\bar{D}^{0}$ mixing.

In the diagram on the right of Fig. 1.4 the intermediate states are the massive $W$ bosons that, given the mass of the mixing mesons, will always be off-shell: the intermediate state is virtual. The interaction can be written as a Fermi four-quark point-like interaction, the intermediate state doesn't travel in space-time and therefore these diagrams belong to the short-distance class, contributing mainly to $\Delta M$. Any contribution of physics beyond the SM belongs to this class. The diagram on the left of Fig. 1.4 is different since the intermediate state is made of light quarks that can travel far from the production point. When this happens, if the distance is comparable to the typical scale of the strong interactions, they can hadronize and form on-shell intermediate states, some examples for the $D^{0}-\bar{D}^{0}$ mixing are reported in Fig. 1.5. These contributions belong to the longdistance class and mainly contribute to $\Delta \Gamma$. The main difference between the long- and short-distance contributions is that in the former QCD becomes a key ingredient while in the latter it does not play a role. 

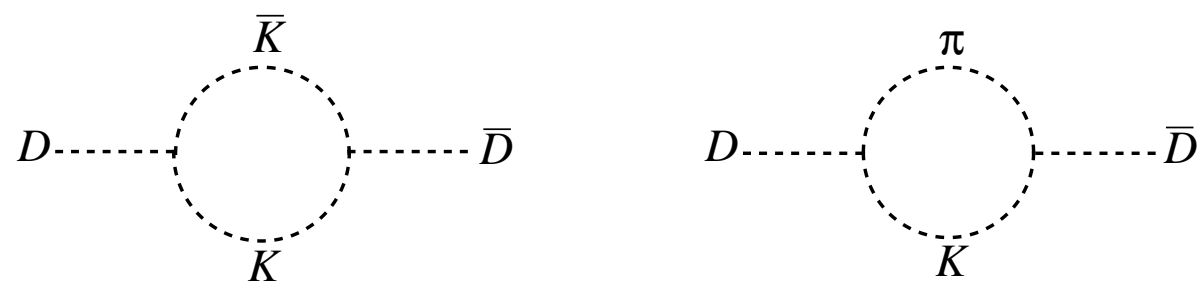

Figure 1.5: Some example of long-distance contributions to $D^{0}-\bar{D}^{0}$ mixing.

The short-distance contributions dominate in the $B^{0}-\bar{B}^{0}$ system, while they are highly suppressed in the $D^{0}-\bar{D}^{0}$ one. The $B^{0}$ system benefits from the presence of a virtual top quark in the loop while in the $D^{0}$ case the bottom quark is not only much lighter than the top, but it is also highly CKM suppressed. Without the contribution of the bottom quark, the GIM mechanism is more effective, hence the contributions of the two lighter quarks $(u, s)$ are also suppressed.

The size of the long-distance contributions is determined by the amount of phase space of the final states in common to the meson and the anti-meson. In the $K^{0}-\bar{K}^{0}$ system this contribution is almost maximal since there is a small number of possible final states for $K^{0}$ and almost all are accessible also to the $\bar{K}^{0}$. In the $B^{0}$ system the situation is the opposite, there is a large number of possible final states for the $B^{0}$ but just a small fraction of them are accessible also to the $\bar{B}^{0}$. In $D^{0}-\bar{D}^{0}$ mixing this is the dominant contribution as a consequence of the large suppression of the short-distance diagrams.

In Tab. 1.2 we report the approximate values for $\Delta M / \Gamma$ and $\Delta \Gamma / 2 \Gamma$ for the four mixing systems and in Fig. 1.6 we show the probability in units of the lifetime to change the flavor (from Eq. (1.45)) or not (from Eq. (1.46)).

\begin{tabular}{c|cc}
\hline \hline system & $\Delta M / \Gamma$ & $\Delta \Gamma / 2 \Gamma$ \\
\hline$K^{0}-\bar{K}^{0}$ & -0.95 & 0.99 \\
$D^{0}-\bar{D}^{0}$ & $5 \times 10^{-3}$ & $5 \times 10^{-3}$ \\
$B^{0}-\bar{B}^{0}$ & 0.77 & $5 \times 10^{-3}$ \\
$B_{s}-\bar{B}_{s}$ & 26 & 0.15 \\
\hline \hline
\end{tabular}

Table 1.2: Approximate values of $\Delta M / \Gamma$ and $\Delta \Gamma / 2 \Gamma$ for the four mixing systems.

For all the systems except the $D^{0}-\bar{D}^{0}$ one, the difference between the $e^{-\Gamma t}$ black line and the flavor-unchanged blue line is visible. In the $D^{0}-\bar{D}^{0}$ plot, on the other hand, the difference is not appreciable, and, in order to see the flavor-changed red line a logarithmic scale with eight orders of magnitude range is needed. This striking difference is the reason why a measurement of mixing in the $D^{0}-\bar{D}^{0}$ system is a high precision measurement, and only with the latest datasets of BABAR, Belle and CDF, it was possible to be sensitive to it.

Although the SM long-distance contributions are dominant, and the predictions are com- 

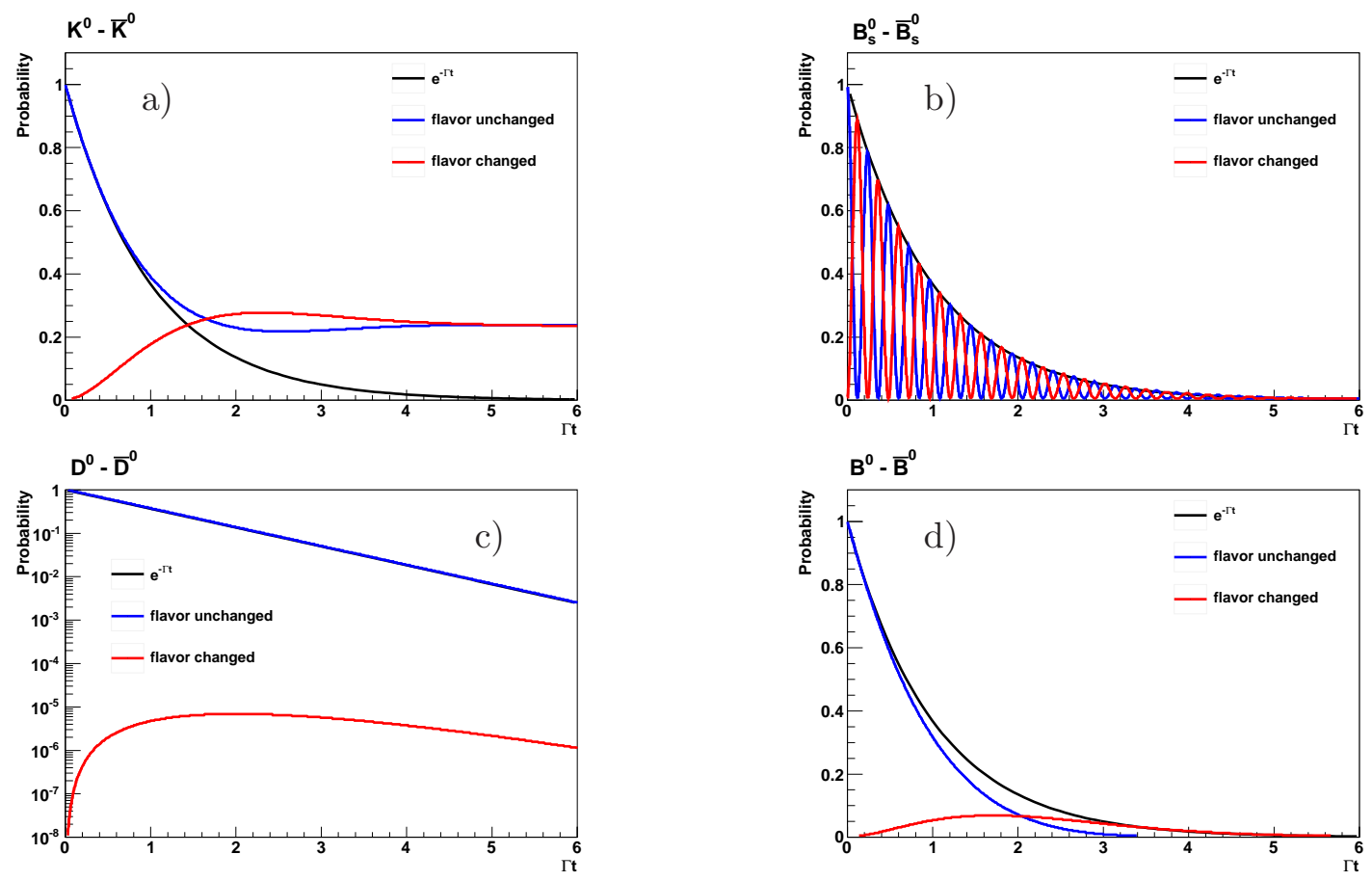

Figure 1.6: Probability of changing the flavor (red line), non changing it (blue) and the $e^{-\Gamma t}$ function (black) for the four SM mixing systems: a) $K^{0}-\bar{K}^{0}$, b) $B_{s}-\bar{B}_{s}$, c) $D^{0}-\bar{D}^{0}$ (note the logarithmic scale), d) $B^{0}-\bar{B}^{0}$.

plicated to compute, as discussed in the following, the $D^{0}$ is unique among the mixing systems, since it is the only one made of up-type quarks, hence the only one for which the virtual quarks are down-type. Therefore mixing in the $D^{0}-\bar{D}^{0}$ system brings complementary information and could be, in principle, sensitive to a different New Physics sector. This is true also for what concerns $C P$ violation.

\section{SM Predictions for the $D^{0}-\bar{D}^{0}$ System}

$D^{0}$ mixing is dominated by the long-distance contributions that are not perturbative in essence. There are two approaches to perform these kind of calculations [28, 29] but none of them provides accurate predictions on $\Delta M / \Gamma$ and $\Delta \Gamma / 2 \Gamma$.

The first approach is the "inclusive" one, based on the Operator Product Expansion (OPE). This approach is based on the assumption that the mass of the charm quark $m_{c}$ is sufficiently large compared to the typical scale of the strong interaction $\Lambda . \Delta M$ and $\Delta \Gamma$ are expanded in terms of the matrix elements of local operators in powers of $\Lambda / m_{c}$. Since the charm quark is relatively heavy, $\Lambda / m_{c}$ is not small enough and the truncation of the series causes a non-negligible error.

A second possible approach is the "exclusive" one, and it consists in summing over all the possible hadronic states. The problem with this approach is that the $D^{0}$ is not light 
enough to be dominated by a few number of final states. Moreover, in order to perform these calculations, the different contributions need to be calculated with high precision because there can be cancellations within a $S U(3)$ multiplet. The knowledge of the strong phases is also important.

As a consequence, the SM predictions for mixing (shown in Fig. 1.7) and for $C P V$ are affected by large theory uncertainties. The interpretation of the current measurements, in particular the evidence of $C P V$, is therefore not straightforward.

Standard Model mixing predictions

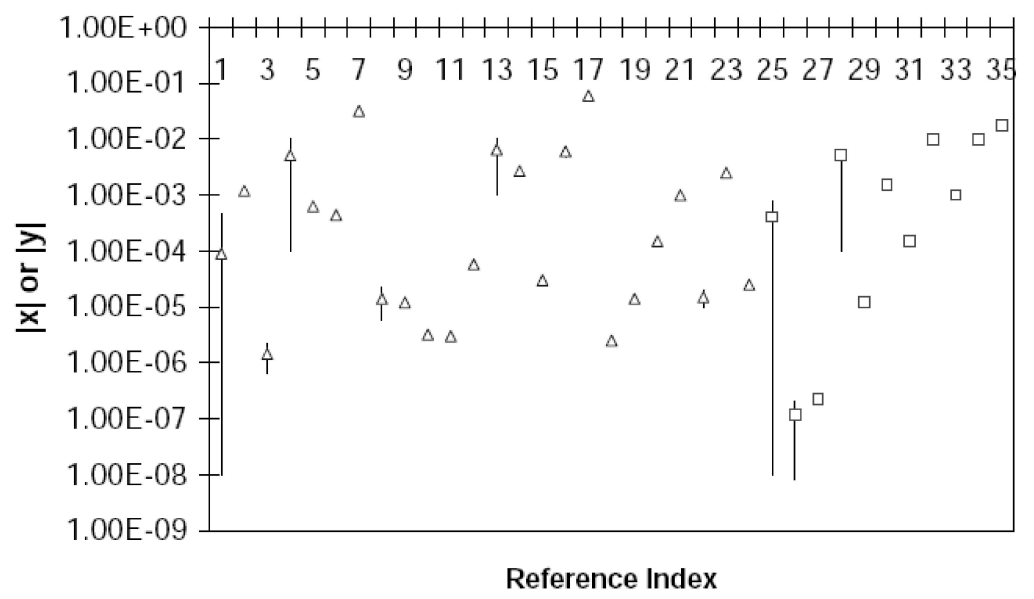

Figure 1.7: SM predictions for the mixing parameter $x=\Delta M / \Gamma$ and $y=\Delta \Gamma / 2 \Gamma$. For the index see [30].

\subsection{Mixing and $C P$ Violation with the Lifetime Ratio in $D^{0}$ Decays}

The $D^{0}$ and $\bar{D}^{0}$ flavor eigenstates are superpositions of the mass eigenstates $D_{1}$ and $D_{2}$, as in (1.32),

$$
\begin{aligned}
& \left|D_{1}\right\rangle=p\left|D^{0}\right\rangle+q\left|\bar{D}^{0}\right\rangle \\
& \left|D_{2}\right\rangle=p\left|D^{0}\right\rangle-q\left|\bar{D}^{0}\right\rangle,
\end{aligned}
$$

$D_{i}$ has a mass $M_{i}$ and a width $\Gamma_{i}$. We use the phase convention $C P\left|D^{0}\right\rangle=+\left|\bar{D}^{0}\right\rangle$, and in the case of no $C P V, D_{1}$ is the $C P$-even eigenstate. The mixing parameters $x_{\mathrm{D}}$ and $y_{\mathrm{D}}$ that describe the $D^{0}-\bar{D}^{0}$ oscillations are defined as:

$$
x_{\mathrm{D}}=\frac{M_{1}-M_{2}}{\Gamma} \quad, \quad y_{\mathrm{D}}=\frac{\Gamma_{1}-\Gamma_{2}}{2 \Gamma} .
$$


Let's consider $C P$-even final states, in particular $f=h^{+} h^{-}, h=K, \pi$. If we neglect second-order terms in $x_{\mathrm{D}} \Gamma t$ and $y_{\mathrm{D}} \Gamma t$, the decay time distributions in (1.50) and (1.51) can be treated as exponentials with an effective lifetime [31]:

$$
\begin{array}{lll}
\Gamma\left(D^{0}(t) \rightarrow f\right) \propto e^{-t / \tau_{h h}^{+}} \quad \text { with } & \left(\tau_{h h}^{+}\right)^{-1}=\Gamma\left[1+y_{\mathrm{D}} \Re\left(\lambda_{h h}\right)-x_{\mathrm{D}} \Im\left(\lambda_{h h}\right)\right], \\
\Gamma\left(\bar{D}^{0}(t) \rightarrow f\right) \propto e^{-t / \bar{\tau}_{h h}^{+}} \quad \text { with } \quad\left(\bar{\tau}_{h h}^{+}\right)^{-1}=\Gamma\left[1+y_{\mathrm{D}} \Re\left(\lambda_{h h}^{-1}\right)-x_{\mathrm{D}} \Im\left(\lambda_{h h}^{-1}\right)\right] .
\end{array}
$$

The "+" sign associated with the lifetime parameters indicates that the $C P$ eigenstate is $C P$-even, while the bar recalls the flavor of the neutral $D$ meson. To better understand the effects of $C P$ violation we introduce two more parameters, one describing $C P V$ in decay $\left(A_{D}^{f}\right)$ and a second one in mixing $\left(A_{M}\right)$ :

$$
\begin{aligned}
A_{D}^{f} & =\frac{\left|A_{f} / \bar{A}_{f}\right|^{2}-\left|\bar{A}_{\bar{f}} / A_{\bar{f}}\right|^{2}}{\left|A_{f} / \bar{A}_{f}\right|^{2}+\left|\bar{A}_{\bar{f}} / A_{\bar{f}}\right|^{2}}, \\
A_{M} & =\frac{|q / p|^{2}-|p / q|^{2}}{|q / p|^{2}+|p / q|^{2}} .
\end{aligned}
$$

Since $f=h^{+} h^{-}$then $|\bar{f}\rangle=C P|f\rangle=+|f\rangle$. Noting that there is no strong phase in $\lambda_{h h}$ since the final state is a $C P$ eigenstate, we can express $\lambda_{h h}$ in terms of $A_{D}^{h h}, A_{M}$ and the $C P$-violating phase $\phi_{h h}$ :

$$
\lambda_{h h}=\left[\frac{1-A_{D}^{h h}}{1+A_{D}^{h h}} \frac{1+A_{M}}{1-A_{M}}\right]^{1 / 4} e^{i \phi_{h h}} .
$$

Expanding Eqs. (1.70) and (1.71) and retaining only terms up to the first order in $A_{D}^{h h}$ and $A_{M}$, we obtain

$$
\begin{aligned}
\left(\tau_{h h}^{+}\right)^{-1} \simeq & \Gamma\left[1+\left(y_{\mathrm{D}} \cos \phi_{h h}-x_{\mathrm{D}} \sin \phi_{h h}\right)+\frac{1}{2}\left(A_{M}-A_{D}^{h h}\right)\left(y_{\mathrm{D}} \cos \phi_{h h}-x_{\mathrm{D}} \sin \phi_{h h}\right)\right. \\
& \left.-\frac{1}{4} A_{M} A_{D}^{h h}\left(y_{\mathrm{D}} \cos \phi_{h h}-x_{\mathrm{D}} \sin \phi_{h h}\right)\right] \\
\left(\bar{\tau}_{h h}^{+}\right)^{-1} \simeq & \Gamma\left[1+\left(y_{\mathrm{D}} \cos \phi_{h h}+x_{\mathrm{D}} \sin \phi_{h h}\right)-\frac{1}{2}\left(A_{M}-A_{D}^{h h}\right)\left(y_{\mathrm{D}} \cos \phi_{h h}+x_{\mathrm{D}} \sin \phi_{h h}\right)\right. \\
& \left.-\frac{1}{4} A_{M} A_{D}^{h h}\left(y_{\mathrm{D}} \cos \phi_{h h}+x_{\mathrm{D}} \sin \phi_{h h}\right)\right]
\end{aligned}
$$

Combining the widths defined above we obtain the two observables $y_{C P}$ and $\Delta Y$ that, in general, depend on the final state because of the $C P V$ parameters $A_{D}^{h h}$ and $\phi_{h h}$ :

$$
\begin{aligned}
y_{C P}^{h h} & =\frac{1}{2 \Gamma}\left[\frac{1}{\tau_{h h}^{+}}+\frac{1}{\bar{\tau}_{h h}^{+}}\right]-1 \simeq \frac{\tau_{D^{0}}}{2}\left[\frac{1}{\tau_{h h}^{+}}+\frac{1}{\bar{\tau}_{h h}^{+}}\right]-1, \\
\Delta Y^{h h} & =\frac{1}{2 \Gamma}\left[\frac{1}{\tau_{h h}^{+}}-\frac{1}{\bar{\tau}_{h h}^{+}}\right] \simeq \frac{\tau_{D^{0}}}{2}\left[\frac{1}{\tau_{h h}^{+}}-\frac{1}{\bar{\tau}_{h h}^{+}}\right],
\end{aligned}
$$


the average width $\Gamma$ is proportional to the inverse of the $D^{0}$ lifetime, $\tau_{D^{0}}$, if $\Delta \Gamma \ll 1$ and neglecting $C P V$ in the mixing.

These quantities are directly related to the fundamental parameters that govern mixing and $C P V$ in the charm sector:

$$
\begin{aligned}
y_{C P}^{h h} & =y_{\mathrm{D}} \cos \phi_{h h}-\frac{1}{2}\left[A_{M}+A_{D}^{h h}\right] x_{\mathrm{D}} \sin \phi_{h h}-\frac{1}{4} A_{M} A_{D}^{h h} y_{\mathrm{D}} \cos \phi_{h h}, \\
\Delta Y^{h h} & =-x_{\mathrm{D}} \sin \phi_{h h}+\frac{1}{2}\left[A_{M}+A_{D}^{h h}\right] y_{\mathrm{D}} \cos \phi_{h h}+\frac{1}{4} A_{M} A_{D}^{h h} x_{\mathrm{D}} \sin \phi_{h h} .
\end{aligned}
$$

Both $y_{C P}$ and $\Delta Y$ are zero if there is no $D^{0}-\bar{D}^{0}$ mixing. Otherwise, a non-zero value of $y_{C P}$ determines mixing and a non-zero value of $\triangle Y$ determines $C P V$.

In the charm sector, the CKM elements involved belong to the Cabibbo submatrix, therefore we can assume that the weak phase $\phi_{h h}$ does not depend on the final state: $\phi_{h h}=\phi[31]$. As stated earlier, if direct $C P V$ has a significant effect, then the values of $y_{C P}$ and $\Delta Y$ depend on the final state. In this analysis we assume that the effect of direct $C P V$ is negligible in the decays to $C P$ eigenstates; therefore $\tau_{K K}^{+}=\tau_{\pi \pi}^{+}$(and $\bar{\tau}_{K K}^{+}=\bar{\tau}_{\pi \pi}^{+}$). In Eqs. (1.75) and (1.76) this means neglecting the linear terms in $A_{D}^{h h}$. Assuming that $A_{D}^{h h}$ and $y_{\mathrm{D}}$ are both $\mathcal{O}(1 \%)$ and $\phi_{h h}=0$, the neglected term is $\mathcal{O}\left(10^{-4}\right)$, beyond any current experimental sensitivity.

Under the above assumptions, Eqs. (1.75) and (1.76) simplify to:

$$
\begin{aligned}
& \left(\tau^{+}\right)^{-1} \simeq \Gamma\left[1+\left(y_{\mathrm{D}} \cos \phi-x_{\mathrm{D}} \sin \phi\right)+\frac{A_{M}}{2}\left(y_{\mathrm{D}} \cos \phi-x_{\mathrm{D}} \sin \phi\right)\right] \\
& \left(\bar{\tau}^{+}\right)^{-1} \simeq \Gamma\left[1+\left(y_{\mathrm{D}} \cos \phi+x_{\mathrm{D}} \sin \phi\right)-\frac{A_{M}}{2}\left(y_{\mathrm{D}} \cos \phi+x_{\mathrm{D}} \sin \phi\right)\right]
\end{aligned}
$$

while the observables become:

$$
\begin{aligned}
& y_{C P}=y_{\mathrm{D}} \cos \phi-\frac{A_{M}}{2} x_{\mathrm{D}} \sin \phi, \\
& \Delta Y=-x_{\mathrm{D}} \sin \phi+\frac{A_{M}}{2} y_{\mathrm{D}} \cos \phi .
\end{aligned}
$$

\subsection{Experimental Status of the Observables}

The B-Factories and the Tevatron experiments have accumulated sufficient statistics to observe mixing in the $D^{0}-\bar{D}^{0}$ system. The $\mathrm{LHCb}$ experiment has also produced its first measurement of mixing and $C P V$ in the charm sector. The world averages of the mixing and $C P$ violating parameters computed by the Heavy Flavor Averaging Group (HFAG) [32] are reported in Fig. 1.8.

The experimental observations of mixing are consistent with the upper range of predictions 

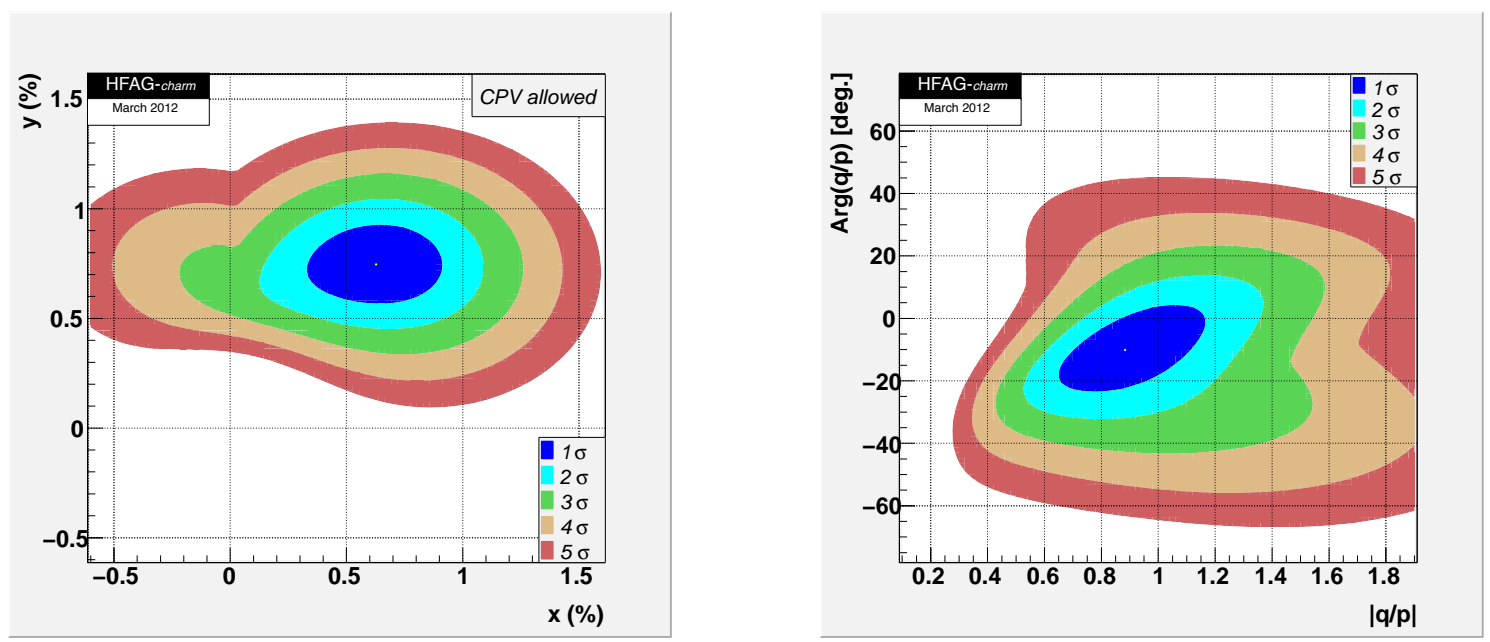

Figure 1.8: World Averages for the mixing parameters $x_{D}$ and $y_{D}$ (left) and the $C P V$ parameters $|q / p|$ and its phase, by the HFAG [32].

from the Standard Model,

$$
\begin{aligned}
& x_{\mathrm{HFAG}}=\left(0.89_{-0.27}^{+0.26}\right) \% \\
& y_{\mathrm{HFAG}}=\left(0.75_{-0.18}^{+0.17}\right) \%
\end{aligned}
$$

For what concerns our observables, $y_{C P}$ and $\Delta Y$, we should point out that HFAG and experiments different from $B A B A R$ (instead of $\Delta Y$ ) use the following parameter sensitive to $C P V A_{\Gamma}$

$$
A_{\Gamma}=\frac{\bar{\tau}_{h h}^{+}-\tau_{h h}^{+}}{\bar{\tau}_{h h}^{+}+\tau_{h h}^{+}}=\frac{\Delta Y}{1+y_{C P}} .
$$

Moreover HFAG neglects the factor $1 /\left(1+y_{C P}\right)$ when it relates the observable $A_{\Gamma}$ to the fundamental mixing and $C P V$ parameters $x_{\mathrm{D}}, y_{\mathrm{D}}$ and $\phi$. In Fig. 1.9 we summarize the measurements of $y_{C P}$ and $A_{\Gamma}$ from BABAR, BELLE and LHCb and the world averages computed by HFAG.

The combined values are:

$$
\begin{aligned}
y_{C P} & =(1.064 \pm 0.209) \% \\
A_{\Gamma} & =(0.026 \pm 0.231) \%
\end{aligned}
$$

No evidence of $C P V$ is found in the parameter $A_{\Gamma}$. Mixing is measured at the $1 \%$ level with the parameter $y_{C P}$.

Each $C P V$ observable has a contribution from direct $C P V$, i.e. from an irreducible phase in the $\Delta F=1$ decay amplitude, and indirect $C P V$, i.e. from an irreducible phase in the 

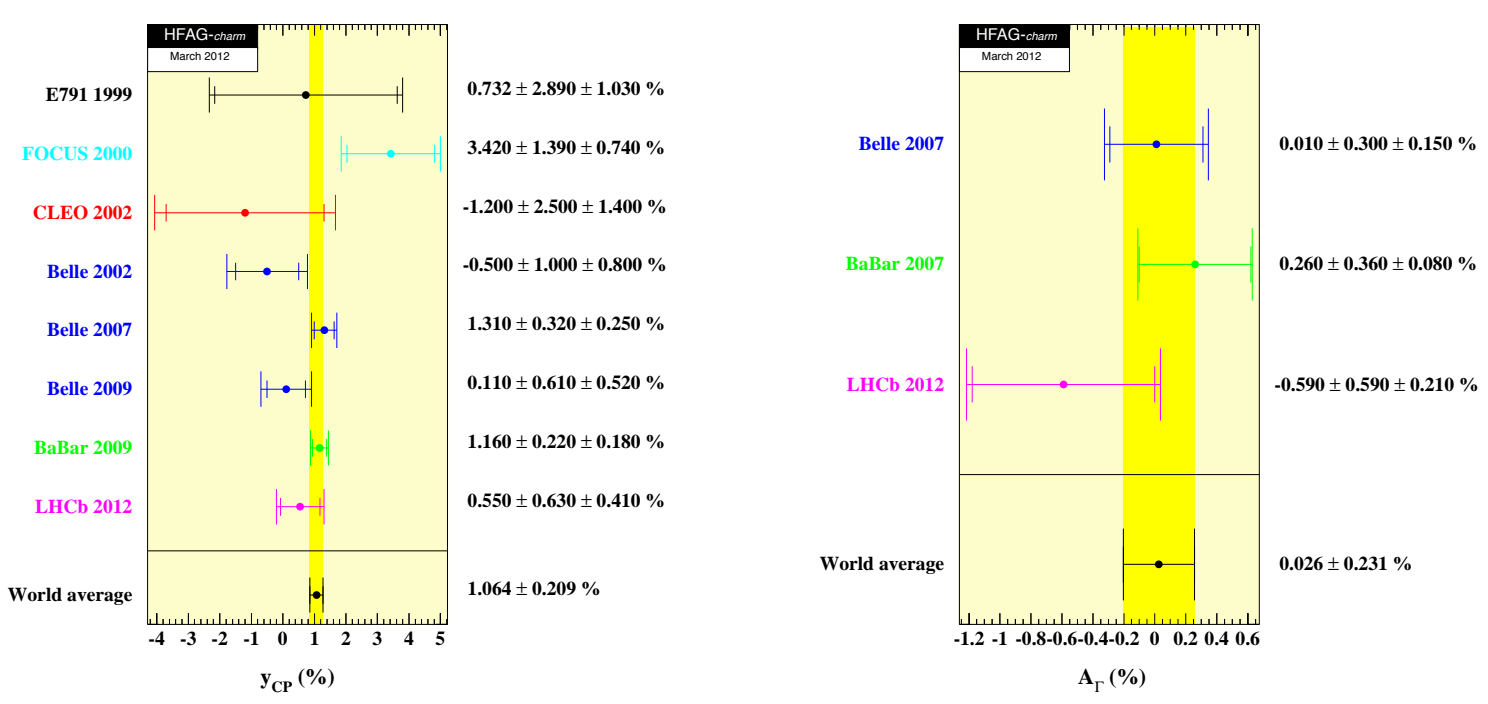

Figure 1.9: Combination of the measurements for $y_{C P}$ and $A_{\Gamma}$ by the HFAG [32].

$\Delta F=2$ mixing transitions. In Fig. 1.10 the direct and indirect $C P V$ contributions have been disentangled by the HFAG.

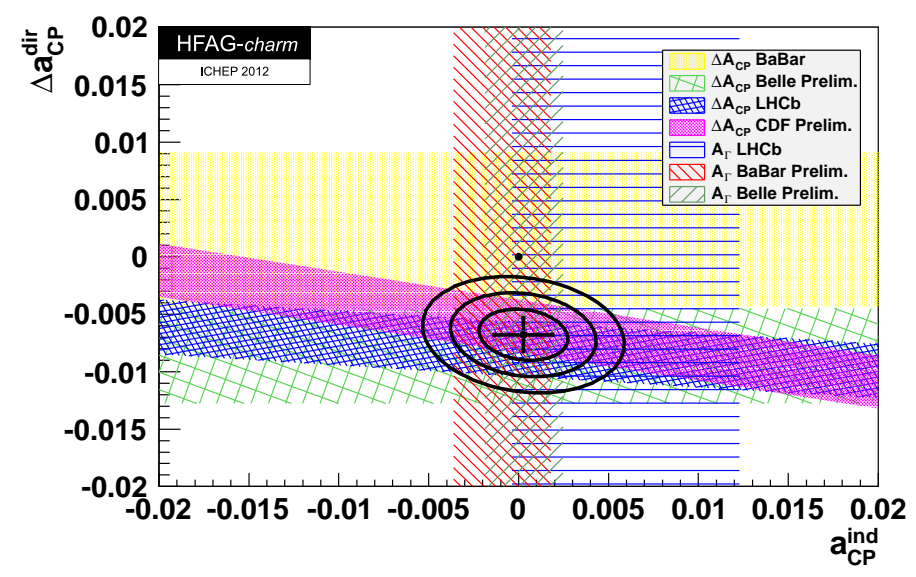

Figure 1.10: Combination of the measurements for direct and indirect $C P V$ in the charm sector by the HFAG [32].

$C P V$ in the charm sector is excluded at a confidence level of $2 \times 10^{-5}$, determined primarily from the direct $C P V$ contribution, that is at the level of $0.7 \%$. 



\section{Chapter 2}

\section{The BABAR Experiment at the B-Factory PEP-II}

The BABAR experiment was installed at the Stanford Linear Accelerator Center (SLAC), California. It was designed and built by a large international team of scientists and engineers in the 90s, with a comprehensive physics program consisting of the systematic measurement of $C P$ violation in the $B$ meson system, precision measurements of decays of bottom and charm mesons, and of the $\tau$ lepton, and search for rare processes. The $B A B A R$ experiment consists of a detector [33] built around the interaction region of the high luminosity $e^{+} e^{-}$collider PEP-II [34]. BABAR started taking data in May 1999 and finished in April 2008. In this chapter the main features of the final design and performance of PEP-II and BABAR are described.

\subsection{The PEP-II B Factory}

The PEP-II $B$ Factory is an asymmetric-energy $e^{+} e^{-}$collider designed to operate at a center-of-mass energy of $10.58 \mathrm{GeV}$, corresponding to the mass of the $\Upsilon(4 S)$ vector meson resonance (see Fig. 2.1). The luminosity $\mathcal{L}$ of the machine depends on the careful tuning of several parameters. This dependence is expressed as:

$$
\mathcal{L}=\frac{n f N_{1} N_{2}}{A}
$$

where $n$ is the number of bunches in a ring, $f$ is the bunch crossing frequency, $N_{1}$ and $N_{2}$ are the total number of particles in each bunch, and $A$ is their overlap cross sectional area $\left(A=4 \pi \sigma_{x} \sigma_{y}\right)$. The design peak luminosity was foreseen to be $\mathcal{L}=3 \times 10^{33} \mathrm{~cm}^{-2} \mathrm{~s}^{-1}$ and was reached in 2001, the record is $1.2 \times 10^{34} \mathrm{~cm}^{-2} \mathrm{~s}^{-1}$ achieved in 2006 .

The effective cross sections of the main physics processes in PEP-II are listed in Tab. 2.1 ([35]), and for the production of the $\Upsilon(4 S)$ at $\sqrt{s}=10.58 \mathrm{GeV}$ it is about $1.1 \mathrm{nb}$, where 


\begin{tabular}{cc}
\hline \hline Process & Cross-section (nb) \\
\hline$e^{+} e^{-} \rightarrow b \bar{b}$ & 1.099 \\
$e^{+} e^{-} \rightarrow c \bar{c}$ & 1.30 \\
$e^{+} e^{-} \rightarrow s \bar{s}$ & 0.35 \\
$e^{+} e^{-} \rightarrow u \bar{u}$ & 1.39 \\
$e^{+} e^{-} \rightarrow d \bar{d}$ & 0.35 \\
$e^{+} e^{-} \rightarrow e^{+} e^{-}$ & $\sim 53$ \\
$e^{+} e^{-} \rightarrow \mu^{+} \mu^{-}$ & 1.16 \\
$e^{+} e^{-} \rightarrow \tau^{+} \tau^{-}$ & 0.94 \\
\hline \hline
\end{tabular}

Table 2.1: Cross sections of the main physics processes at the $\Upsilon(4 S)$. The cross section for $e^{+} e^{-}$refers to the volume of BABAR electromagnetic calorimeter, which is used to trigger these events. The $b \bar{b}$ cross-section is the ratio of the number of produced $B$ events to luminosity in the On-peak Run 1-5 data sample. The other $q \bar{q}$ cross sections are computed by Jetset7.4 and include radiative corrections.

the $\Upsilon(4 S)$ decays almost exclusively into $B^{0} \bar{B}^{0}$ and $B^{+} B^{-}$pairs.

As shown in Fig. 2.1, at the peak of $\Upsilon(4 S)$ there is a non-negligible amount of $e^{+} e^{-} \rightarrow q \bar{q}$ $(q=u, d, s, c)$ and $e^{+} e^{-} \rightarrow l^{+} l^{-}(l=e, \mu, \tau)$ events. In addition, part of the data is collected at the center-of-mass $(\mathrm{CM})$ energy $40 \mathrm{MeV}$ below the $\Upsilon(4 S)$ peak, where $B \bar{B}$ production is not allowed. This data sample corresponds to about 1/10 of the sample taken at the $\Upsilon(4 S)$ peak and, in the "conventional" BABAR analysis, is used to study non- $B \bar{B}$ background. In this analysis it is included in the signal sample.

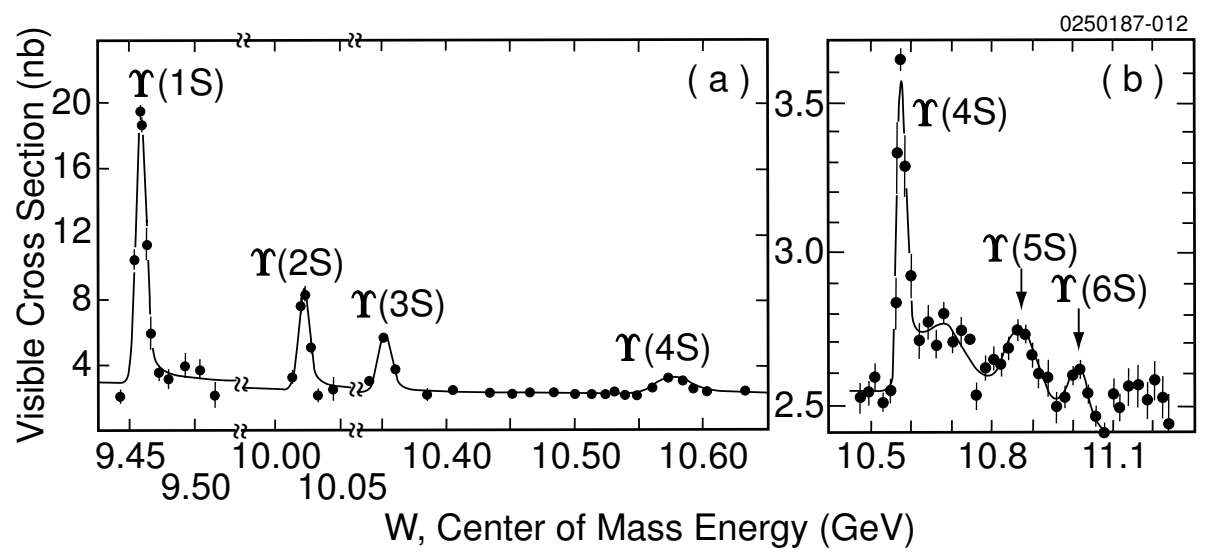

Figure 2.1: (a) The first four S-wave $\Upsilon$ resonances shown with the hadronic cross section versus center-of-mass energy $/ c^{2}$ in the $\Upsilon$ mass region. (b) a magnified region showing the $\Upsilon(4,5,6 S)$ resonances. The $\Upsilon(4 S)$ is the third radial excitation of the ground state. Its larger width is a consequence of the fact that the $\Upsilon(4 S)$ is just above threshold for strongly decaying to $B^{0} \bar{B}^{0}$ and $B^{+} B^{-}$pairs. 


\begin{tabular}{lccc}
\hline \hline Parameters & Units & Design & $\Upsilon(4 S)$ Typical \\
\hline Energy $(E)$ HER/LER & $\mathrm{GeV}$ & $9.0 / 3.1$ & $9.0 / 3.1$ \\
Current $(I)$ HER/LER & $\mathrm{A}$ & $0.75 / 2.15$ & $0.9-1.9 / 1.3-2.6$ \\
number of bunches & - & 1658 & 1732 \\
$\sigma_{x}$ & $\mu \mathrm{m}$ & 110 & 120 \\
$\sigma_{y}$ & $\mu \mathrm{m}$ & 3.3 & 4.1 \\
$\sigma_{z}$ & $\mu \mathrm{m}$ & 11 & $11-12$ \\
$\beta_{y}^{*}$ & $\mathrm{~mm}$ & $15-25$ & $9-10$ \\
$\beta_{x}^{*}$ & $\mathrm{~cm}$ & 50 & $40-105$ \\
$\xi_{y}$ HER/LER & - & $0.03 / 0.03$ & $0.062 / 0.047$ \\
$\xi_{x}$ HER/LER & - & $0.03 / 0.03$ & $0.113 / 0.027$ \\
Bunch Spacing & $\mathrm{ns}$ & 4.2 & 4.2 \\
Luminosity & $10^{33} \mathrm{~cm}^{-2} \mathrm{~s}^{-1}$ & 3 & $4.4-10.4$ \\
\hline \hline
\end{tabular}

Table 2.2: PEP-II beam parameters [36]. HER and LER refer to the high energy $e^{-}$ and low $e^{+}$ring respectively. $\sigma_{x}, \sigma_{y}$, and $\sigma_{z}$ refer to the R.M.S. horizontal, vertical, and longitudinal bunch size at the IP. $\beta_{x, y}^{*}$ is the horizontal and vertical envelope function at the collision point and $\xi_{x, y}$ the tune shift. The peak luminosity is proportional to $E I \xi_{y} / \beta_{y}^{*}$, assuming the product $E \cdot I$ roughly equal for the two beams.

\subsubsection{PEP-II Layout}

In PEP-II, the electron beam of $9.0 \mathrm{GeV}$ collides almost head-on ${ }^{1}$ with the positron beam of $3.1 \mathrm{GeV}$ resulting in a boost for the $\mathrm{CM}$ system of $\beta \gamma \approx 0.56$ in the laboratory reference frame (LAB). This boost is crucial to study the $B$-meson system: it allows one to reconstruct the decay vertex of the two $B$ mesons and to determine their relative decay times, since the average separation between the two $B$ vertexes is $\beta \gamma c \tau \approx 250 \mu \mathrm{m}$. One, can therefore measure the time-dependent decay rates and $C P$-asymmetries.

The different beam energies require a two-ring configuration, as shown in Fig. 2.2. The parameters of PEP-II rings are summarized in Tab. 2.2

Electron and positron are accelerated along the $3 \mathrm{~km}$ long SLAC linear accelerator (LINAC) and accumulated into the two $2.2 \mathrm{~km}$ long storage rings, called HER (High-Energy Ring) and LER (Low-Energy Ring), respectively. A fraction of electrons instead of being delivered to the HER is further accelerated to an energy of $30 \mathrm{GeV}$ and sent to a target where positrons are produced.

In the proximity of the interaction region, the beams are focused by a series of offset quadrupoles (indicated with Q in Fig. 2.5 ) and bent by a pair of samarium-cobalt dipole magnets (B1), which allow the bunches to collide head-on. The B1 dipoles, located at $\pm 21 \mathrm{~cm}$ on each side of the interaction point (IP), and the Q1 quadrupoles, are permanent magnets which operate inside the field of the BABAR superconducting solenoid, while Q2, Q4, and Q5, are located outside or in the fringe field of the solenoid. This configuration is the best compromise between physics and engineering requirements.

\footnotetext{
${ }^{1}$ The crossing angle is $20 \mathrm{mrad}$.
} 


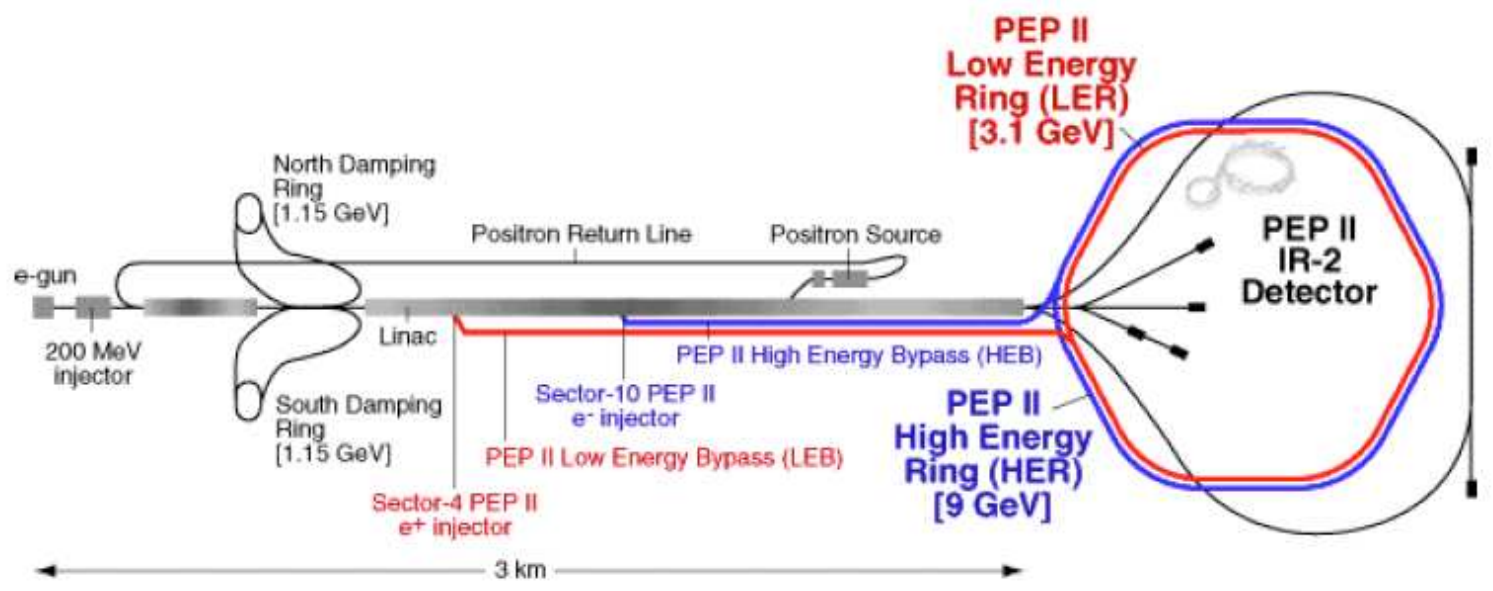

Figure 2.2: The PEP-II asymmetric storage ring and the SLAC linear accelerator. The SLAC LINAC is the injector for PEP-II. The interaction point of PEP-II is at IR-2, where $B A B A R$ is situated.

The interaction region is enclosed in a water-cooled beam pipe consisting of two thin layers of beryllium ( $0.83 \mathrm{~mm}$ and $0.53 \mathrm{~mm}$ ) with a $1.48 \mathrm{~mm}$ water channel in between.

To attenuate synchrotron radiation, the inner surface of the beam pipe is gold-plated (about $4 \mu \mathrm{m}$ ). Beam pipe, permanent magnets, and Silicon Vertex Tracker (SVT) are assembled, aligned, and then enclosed in a $4.4 \mathrm{~m}$ long support tube, which is inserted into the $B A B A R$ detector.

\subsubsection{PEP-II Performances}

PEP-II has delivered luminosity starting from May 1999 till April 2008, and BABAR has recorded a total integrated luminosity of $531 \mathrm{fb}^{-1}$, mostly at the $\Upsilon(4 S)$ resonance peak (also called the on-peak sample) plus small samples around $\Upsilon(2 S)$ and $\Upsilon(3 S)$ ones, as shown in Fig. 2.3.

Some off-peak luminosity has been collected $40 \mathrm{MeV}$ below each resonance peak, and finally an energy scan from $\Upsilon(4 S)$ till $11.2 \mathrm{GeV}$ in steps of $5 \mathrm{MeV}$ was performed, totaling about $54 \mathrm{fb}^{-1}$ of data. PEP-II has largely surpassed its design performance, with a record peak-luminosity of $1.2 \cdot 10^{34} \mathrm{~cm}^{-2} \mathrm{~s}^{-1}$, and a monthly integrated luminosity of 20 $\mathrm{fb}^{-1}$, that is, respectively, about a factor four and six with respect to the expectations. The progress in the instantaneous luminosity is mainly due to the increase of the beam currents and improved focusing and beam orbits. A significant improvement of the order of $50 \%$ of the integrated luminosity has been achieved at the begin of year 2004 with the implementation of a novel mode of operation of PEP-II called trickle injection (see Fig. 2.4). Until the end of 2003, PEP-II typically operated in a series of 40 minute fills during which the colliding beam coasted: at the end of each fill, it took about three to five minutes to replenish the beams for the next fill, and during this period the BABAR data acquisition system had to be turned off because of the high background conditions present 


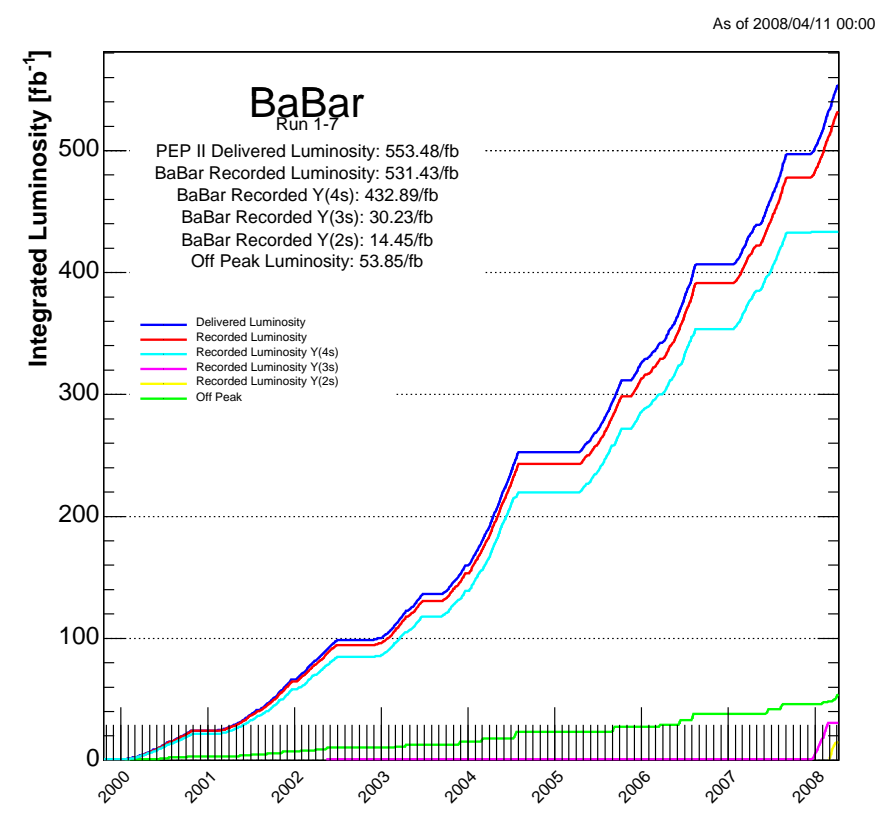

Figure 2.3: Total luminosity delivered by PEP-II from October 1999 to April 2008. The luminosities integrated by $B A B A R$ at different resonances is also shown.

during a fill, affecting detector safety and introducing data acquisition dead-time. With trickle injection there is no "fill" concept and beams are replenished with a small amount of charge to keep the current constant. With the new technique, the BABAR detector could take data uninterrupted resulting in much higher integrated luminosity.

Trickle injection was introduced first in December 2003 in the low energy ring, and in March 2004 it was implemented in the high energy ring. The advantages of this novel mode of operation went beyond just the increase in luminosity. Continuous injection made the storage of particles more stable, so that PEP-II rings were easier to operate and beam losses were far less frequent than with the previous operational mode.

\subsubsection{PEP-II Background}

Beam-generated background causes high single-counting rates, data acquisition dead time, high currents and radiation damage of the BABAR detector and electronics. This results in low data quality and can affect the lifetime of the apparatus. For this reason the background generated by PEP-II was studied in detail and the interaction region was carefully designed. The primary sources of the machine-generated background are:

- synchrotron radiation in the proximity of the interaction region. A strong source of background is due to beam deflections in the interaction region. This component is limited by channeling the radiation out of the BABAR acceptance with a proper 

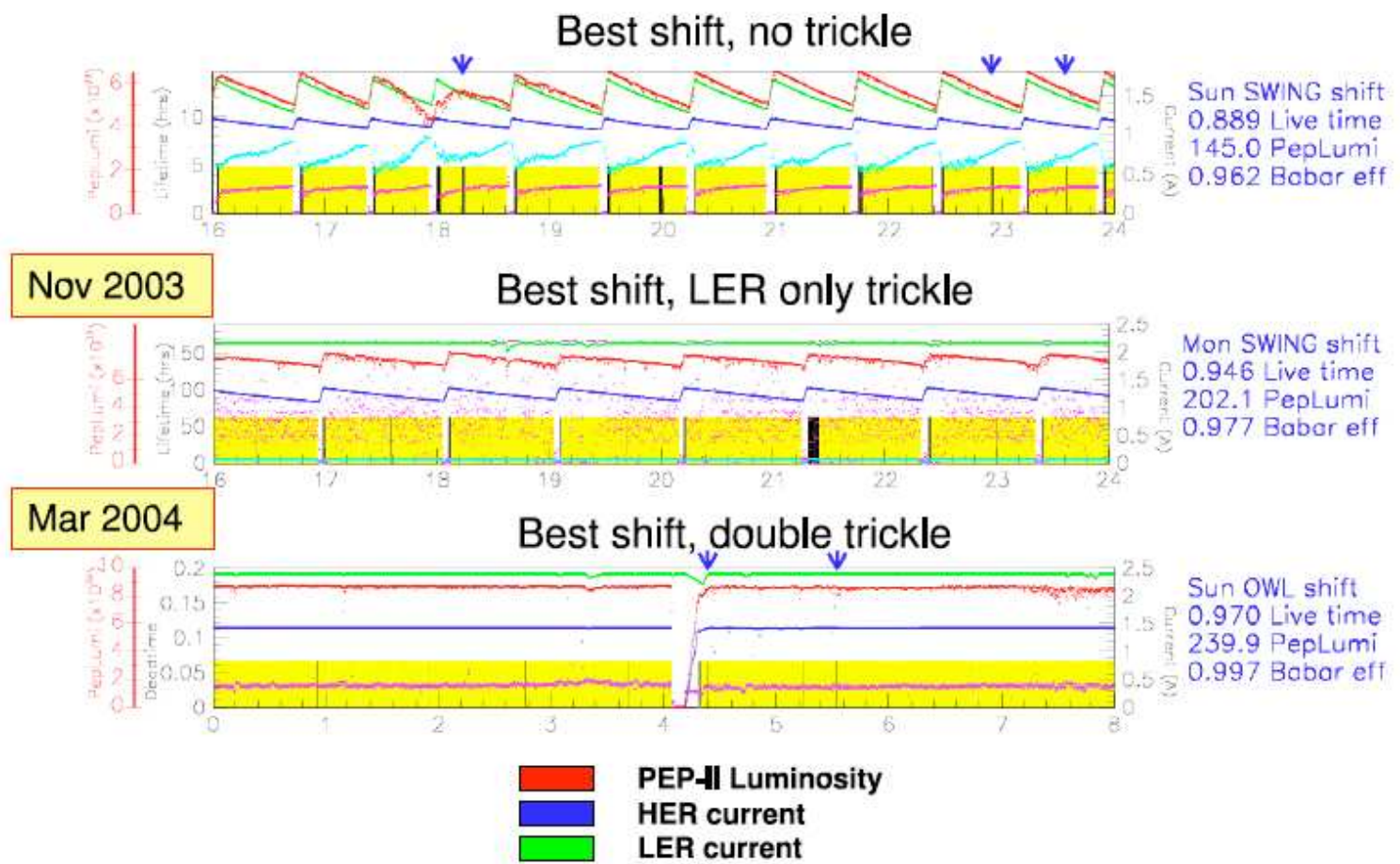

Figure 2.4: Comparison of the best 8-hour periods of data taking for three different modes of operation of PEP-II: no trickle injection (top), trickle injection of the low energy ring (middle), and trickle injection of both beams (bottom).

design of the interaction region and beam orbits, and placing absorbing masks before the detector components;

- interaction between beam particles and residual gas in either ring, originating from beam gas bremsstrahlung and Coulomb scattering. Both types of interaction cause an escape of beam particles from their orbit. This background represents the primary source of radiation damage for the inner vertex detector and the principal background source for the other components. The intrinsic rate of these processes is proportional to the product of the beam current and the residual pressure;

- Electromagnetic shower generated by beam-beam collision. These showers are due to $e^{+} e^{-}$pairs produced by radiative Bhabha scattering and hitting the beam pipe close to the interaction point. This background is proportional to the machine luminosity and it is always monitored.

\subsection{Overview of the BABAR Detector}

The design of the BABAR detector has been optimized for $C P$ violation studies, but it is also well suited for searches of rare decays of $\mathrm{B}, \tau$, and charm physics. To achieve the goal of performing accurate event reconstruction there are many requirements: 


\begin{tabular}{|c|c|c|c|c|c|}
\hline System & $\begin{array}{l}\text { Polar Angle } \\
\text { coverage }(\theta)\end{array}$ & Channels & Layers & Segmentation & Performance \\
\hline SVT & {$[20.1,150.2]^{\circ}$} & $150 \mathrm{~K}$ & 5 & $\begin{array}{l}50-100 \mu \mathrm{m} \text { in } r-\phi \\
100-200 \mu \mathrm{m} \text { in } z\end{array}$ & \multirow{2}{*}{$\begin{array}{l}\sigma_{d 0}=55 \mu \mathrm{m} \\
\sigma_{z 0}=65 \mu \mathrm{m} \\
\sigma_{\phi}=1 \mathrm{mrad} \\
\sigma_{\tan \lambda}=0.001 \\
\sigma_{p_{T}} / p_{T}=0.47 \% \\
\sigma(\mathrm{d} E / \mathrm{d} x)=7.5 \%\end{array}$} \\
\hline $\mathrm{DCH}$ & {$[17.2,152.6]^{\circ}$} & 7,104 & 40 & $\begin{array}{l}6-8 \mathrm{~mm} \\
\text { drift distance }\end{array}$ & \\
\hline DIRC & {$[25.5,141.4]^{\circ}$} & 10,752 & 1 & $\begin{array}{l}35 \times 17 \mathrm{~mm}^{2} \\
(r \Delta \phi \times \Delta r) \\
144 \text { bars }\end{array}$ & $\begin{array}{l}\sigma_{\theta_{C}}=2.5 \mathrm{mrad} \\
\text { per track }\end{array}$ \\
\hline EMC (C) & {$[27.1,140.8]^{\circ}$} & $2 \times 5760$ & 1 & $\begin{array}{l}47 \times 47 \mathrm{~mm}^{2} \\
5760 \text { crystals }\end{array}$ & $\begin{array}{l}\sigma_{E} / E=3.0 \% \\
\sigma_{\phi}=3.9 \mathrm{mrad}\end{array}$ \\
\hline $\operatorname{EMC}(\mathrm{F})$ & {$[15.8,27.1]^{\circ}$} & $2 \times 820$ & & 820 crystals & $\sigma_{\theta}=3.9 \mathrm{mrad}$ \\
\hline$\overline{\mathrm{IFR}(\mathrm{C})}$ & {$[47,123]^{\circ}$} & $22 \mathrm{~K}+2 \mathrm{~K}$ & $19+2$ & $20-38 \mathrm{~mm}$ & $\begin{array}{l}90 \% \mu^{ \pm} \text {eff. } \\
6-8 \% \pi^{ \pm} \text {mis-id }\end{array}$ \\
\hline $\operatorname{IFR}(\mathrm{F})$ & {$[20,47]^{\circ}$} & $14.5 \mathrm{~K}$ & 18 & $28-38 \mathrm{~mm}$ & $\begin{array}{l}\text { (loose selection, } \\
1.5-3.0 \mathrm{GeV} / c \text { ) }\end{array}$ \\
\hline IFR (B) & {$[123,154]^{\circ}$} & $14.5 \mathrm{~K}$ & 18 & $28-38 \mathrm{~mm}$ & \\
\hline
\end{tabular}

Table 2.3: Overview of the coverage, segmentation, and performance of the BABAR detector system. The notation $(\mathrm{C}),(\mathrm{F})$, and (B) refers to the central barrel, forward and backward components of the system, respectively. The detector coverage in the laboratory frame is specified in terms of the polar angle $\theta$. Performance numbers are quoted for $1 \mathrm{GeV} / \mathrm{c}$ particles, except where noted.

- a large acceptance and uniform efficiency, in particular down to small polar angles relative to the boost direction, to avoid particle losses;

- excellent detection efficiency for charged particles down to $60 \mathrm{MeV} / \mathrm{c}$ and for photons down to $25 \mathrm{MeV}$;

- good momentum resolution to kinematically separate signal from background;

- excellent energy and angular resolution for the detection of photons from $\pi^{0}$ and $\eta_{0}$ decays, and from radiative decays in the range from $25 \mathrm{MeV}$ to $4 \mathrm{GeV}$;

- very good vertex resolution, both transverse and parallel to the beam;

- identification of electron and muon over a wide range of momentum, primarily for the detection of semi-leptonic decays used to tag the $B$-meson flavor and for the study of semi-leptonic and rare decays;

- identification of hadrons over a wide range of momentum;

- a highly efficient, selective trigger system with redundancy so as to avoid significant signal losses and systematic uncertainties. 
The BABAR detector (see Fig. 2.5), designed and fabricated by a collaboration of 600 physicists of 75 institutions from 9 countries, meets all these requirements, as will be described in the next sections on this chapter. To take into account the boost of PEP-II and maximize the geometric acceptance, the whole detector is displaced in the forward direction (the direction of the highest energy beam) with respect to the interaction point by $37 \mathrm{~cm}$. An overview of the polar angle $(\theta)$ coverage, the segmentation and the performance of the BABAR detector system is summarized in Tab. 2.3. All the detectors have full acceptance in azimuth $(\phi)$.

The BABAR superconducting solenoid, which produces a $1.5 \mathrm{~T}$ axial magnetic field, contains a set of nested detectors, from inside to outside:

- a five layer (double sided) of silicon vertex detector (SVT),

- a central drift chamber (DCH) for charged particle detection and momentum measurement,

- a ring-imaging Cherenkov radiation detector (DIRC) for charged particles identification,

- a $\mathrm{CsI}(\mathrm{Tl})$ crystal electromagnetic calorimeter (EMC) for the detection of photons and electrons.

Outside the solenoid, the flux return of the is filled with 18 layers of steel, with gaps instrumented initially with resistive plate chambers (RPC) and successively replaced with limited streamer tubes (LST). The instrumented flux return (IFR) allows the separation of muons and charged hadrons, and also detects penetrating neutral hadrons.

As indicated in Fig. 2.5, the right-handed coordinate system is anchored to the main tracking system, the drift chamber, with the $z$-axis coinciding with its principal axis. This axis is offset relative to the beam axis by about $20 \mathrm{mrad}$ in the horizontal plane. The positive $y$-axis points upward and the positive $x$-axis points away from the center of the PEP-II storage rings.

In the following section there is a brief description of each sub-detector and their performance. Further details can be found in [33]. 


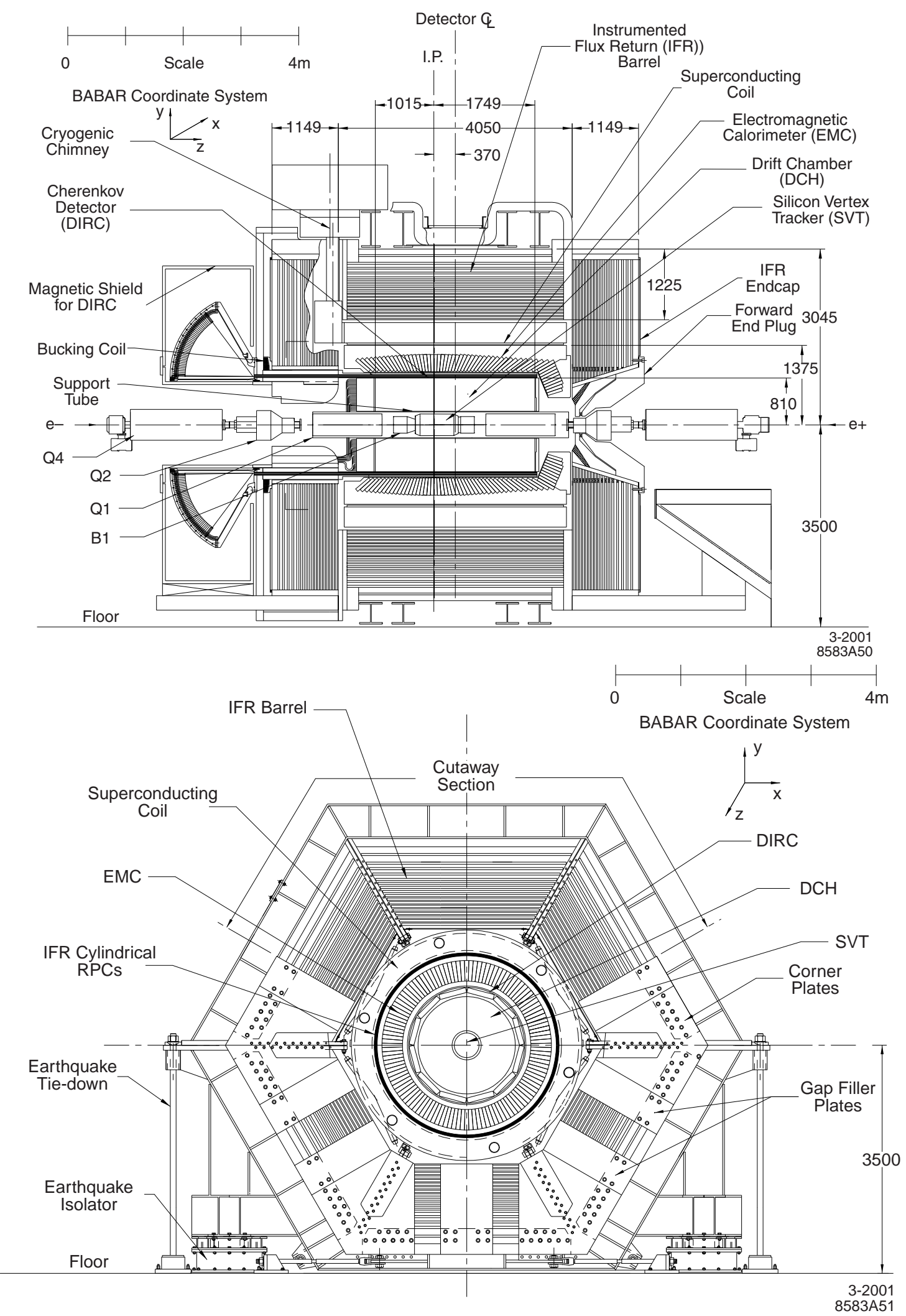

Figure 2.5: Layout of the BABAR detector projected along the beam axis (top) and projected on the plane orthogonal to the beam axis (bottom). 


\subsection{The Silicon Vertex Tracker (SVT)}

The SVT sub-detector provides a precise measurement of the decay vertices and of the charged particle trajectories near the interaction region. The mean vertex resolution along the $z$-axis must be better than $80 \mu \mathrm{m}$ in order to avoid a significant impact on the timedependent $C P$ asymmetry measurements, and a $100 \mu \mathrm{m}$ resolution in the $x-y$ transverse plane is necessary in reconstructing decays of bottom and charm mesons, as well as $\tau$ leptons ${ }^{2}$.

The SVT also provides standalone tracking for particles with transverse momentum too low to reach the outer tracker, like soft pions from $D^{*}$ decays and many charged particles produced in multi-body $B$-meson decays. The choice of a vertex tracker made of five layers of double-sided silicon strip sensors allows a complete track reconstruction even in the absence of drift chamber information.

Finally, the SVT supplies PID information both for low and high momentum tracks. For low-momentum tracks the SVT $\mathrm{d} E / \mathrm{d} x$ is the only PID information available, for high momentum tracks the SVT provides the best measurement of the track angles, required to achieve the designed resolution on the Cherenkov angle measured by the DIRC.

\subsubsection{Detector Layout}

The Silicon Vertex Tracker is composed of five layers of $300 \mu \mathrm{m}$ thick, double-sided microstrip detectors [37]. The total active silicon area is $0.96 \mathrm{~m}^{2}$ and the material traversed by particles at normal incidence is $4 \% X_{0}$. The geometrical acceptance is about $90 \%$ of the solid angle in the CM system.

The silicon detectors and the associated readout electronics are assembled into mechanical units called modules. The inner three layers have six detector modules and are a traditional barrel-style structure. They are placed next to the interaction region, at radii 3.3, 4.0, and $5.9 \mathrm{~cm}$ from the beam axis (see Fig. 2.6 and Fig. 2.7), and provide an accurate measurement of the track impact parameters along $z$ and in the $x-y$ plane.

The outer two layers, composed of 16 and 18 modules, have a peculiar arch structure to reduce the incident angles of particles going in the forward and backward directions, and their barrel parts are placed at radii between 12.7 and $14.6 \mathrm{~cm}$ from the beam axis. They allow an accurate polar angle measurement and, along with the inner three layers, enable standalone tracking for particles with low transverse momentum. Full azimuthal coverage is obtained by partially overlapping adjacent modules, which is also advantageous for alignment. The polar angle coverage in the laboratory frame is $20.1^{\circ}<\theta_{L A B}<150.2^{\circ}$. Each silicon detector consists of a high-resistivity $n^{-}$bulk implanted with $p^{+}$strips on

\footnotetext{
${ }^{2}$ For example, in decays of the type $B^{0} \rightarrow D^{+} D^{-}$, the separation of the two $D$ vertices is important. The distances between the two $D$ 's in the $x-y$ plane for this decay is typically $\sim 275 \mu \mathrm{m}$. Hence, the SVT needs to provide a resolution of about $\sim 100 \mu \mathrm{m}$ in the plane perpendicular to the beam line.
} 


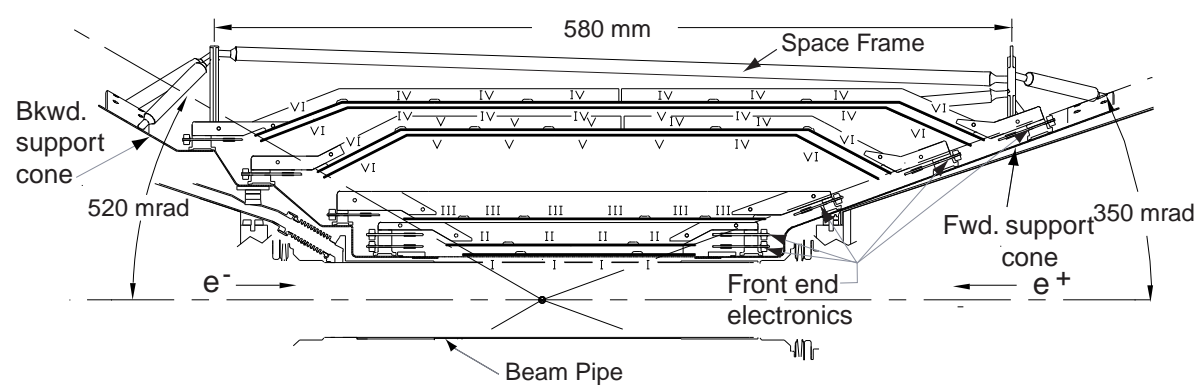

Figure 2.6: Longitudinal section of the SVT detector.

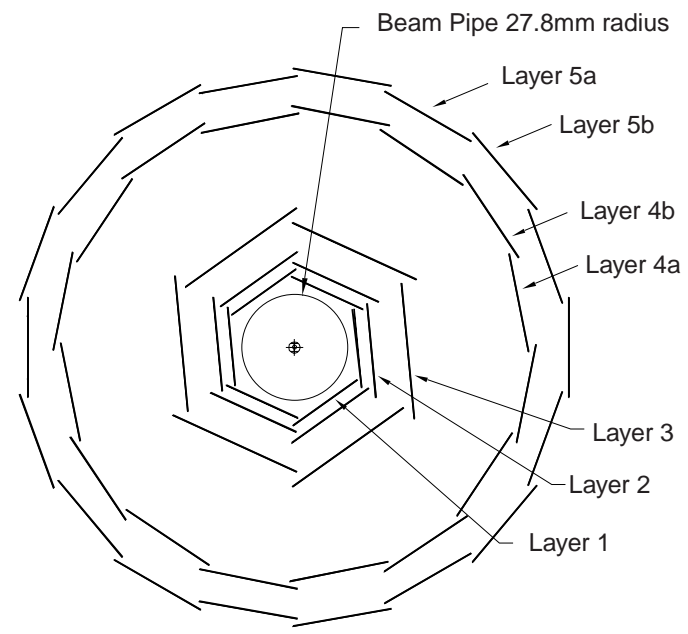

Figure 2.7: Transverse section of the SVT detector.

one side and orthogonally-orientated $n^{+}$strips on the other side. The strips are ACcoupled to the electronics via integrated decoupled capacitors, and the strip pitch varies from 50 to $210 \mu \mathrm{m}$ depending on the layer. The detector is operated in reverse mode at full depletion, with bias voltage $\left(V_{\text {bias }}\right)$ typically $10 \mathrm{~V}$ higher than the depletion voltage $\left(V_{\text {depl }}\right)$, which ranges between $25-35 \mathrm{~V}$. The strips are biased through polysilicon resistors $(4-20 \mathrm{M} \Omega$ ) and the detector active area is surrounded by an implanted guard ring that collects the edge currents and shapes the electric field in the active region. Strips are connected through fanout circuits to the low noise front-end electronics which are located outside the acceptance of the detectors. Each module is electrically divided in four readout sections.

The SVT is located inside a $\sim 4.5$ m-long carbon-fiber support tube. Since the SVT is the inner detector, it is very important that the total amount of material is kept at a minimum, to minimize the multiple scattering. The support tube is made of a carbon-fiber epoxy composite with a thickness of $0.79 \%$ of a radiation length. 


\subsubsection{Detector Performance}

\section{Hit Efficiency and Resolution}

The SVT hit efficiency is determined by comparing the number of hits found by a halfmodule and assigned to a reconstructed track to the number of tracks crossing the active area of the module. Excluding the readout sections which were defective, the combined hardware and software efficiency is measured to be about $97 \%$.

Fig. 2.8 shows the measured SVT spatial hit resolution in $z$ and $r-\phi$ for the five layers, as a function of the track incident angle with respect to the silicon wafer plane. The spatial resolution of SVT hits is determined by measuring the distance between the track trajectory and the hit, using high-momentum tracks in two-prong events. The uncertainties due to the track trajectory subtracted to obtain the hit resolution, which varies between 15 and $50 \mu \mathrm{m}$.
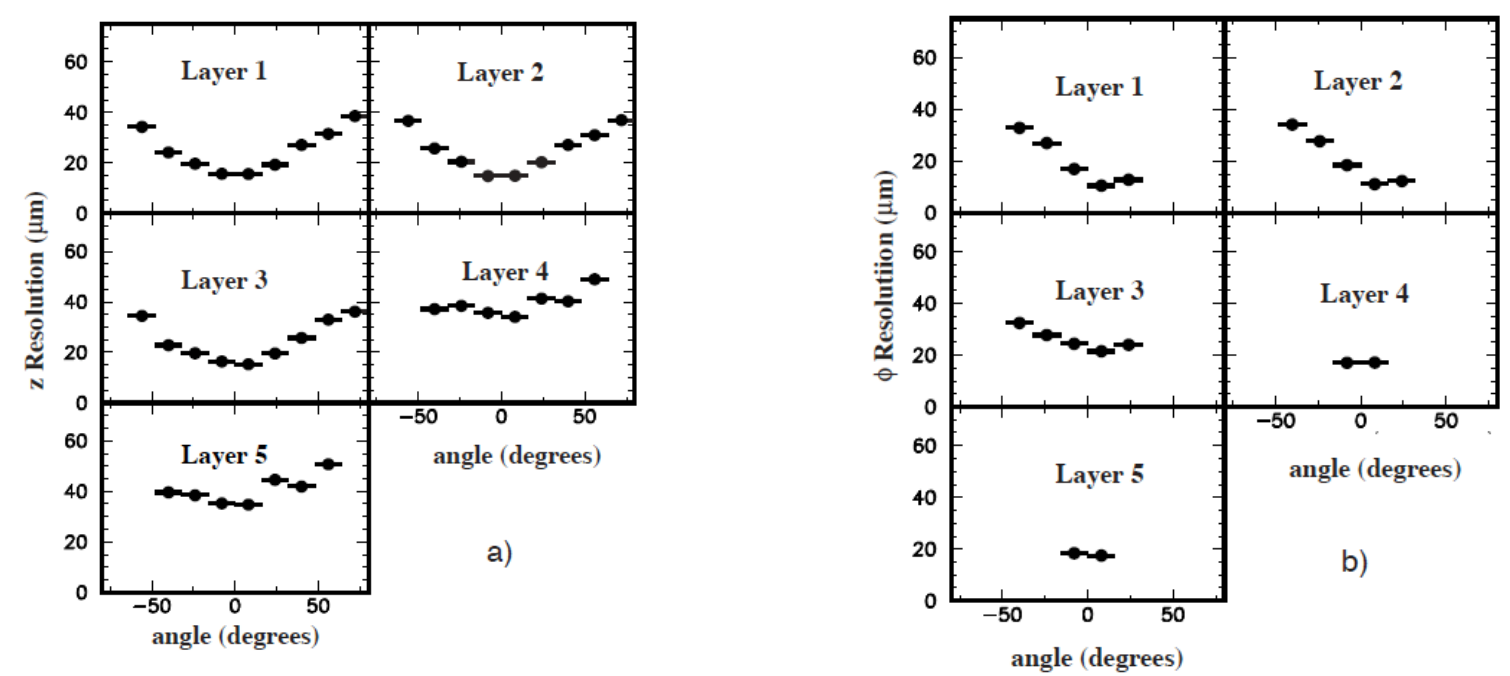

Figure 2.8: SVT hit resolutions in the $z$ (a) and $\phi(\mathrm{b})$ coordinates in $\mu \mathrm{m}$, plotted as a function of track incident angle in degrees. Each plot shows a different layer of the SVT. The plots in $\phi$ coordinate for layers 1-3 are asymmetric around $\phi=0$ because of the "pinwheel" design of the inner layers. There are fewer points in the $\phi$ resolution plots for the outer layers as they subtend smaller angles than the inner layers.

\section{$\mathrm{d} E / \mathrm{d} x$ Resolution}

Limited particle identification (PID) for low-momentum particles that do not reach the drift chamber and the Cherenkov detector is provided by the SVT through the measurement of the specific ionization loss, $\mathrm{d} E / \mathrm{d} x$, as derived from the total charge deposited in each silicon layer. It is computed as a truncated mean from the lowest $60 \%$ of the individual $\mathrm{d} E / \mathrm{d} x$ measurements for tracks with at least 4 associated SVT hits. The resulting SVT $\mathrm{d} E / \mathrm{d} x$ distribution as a function of momentum is shown in Fig. 2.9 [38]. The 
superimposed Bethe-Bloch curves for the individual particle species have been determined using various particle control samples, and a $2 \sigma$ separation between kaons and pions can be achieved up to momenta of $500 \mathrm{MeV} / \mathrm{c}$.

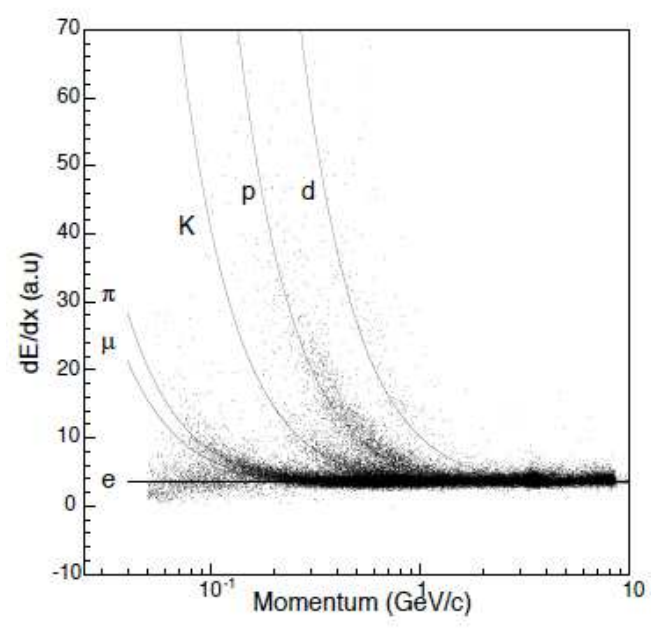

Figure 2.9: Energy loss per unit length $(\mathrm{d} E / \mathrm{d} x)$ as measured in the SVT as a function of momentum. The enhancement of protons is due to beam-gas interactions. The vertical scale is arbitrary.

\subsection{The Drift CHamber (DCH)}

The DCH sub-detector is the main tracking device for charged particles with transverse momenta $p_{T}$ above $120 \mathrm{MeV} / \mathrm{c}$, providing the measurement of $p_{T}$ from the curvature of the particle trajectory inside the solenoid. The DCH also allows the reconstruction of secondary vertices outside the SVT volume, such as those from $K_{S}^{0} \rightarrow \pi^{+} \pi^{-}$decays. For this purpose, the chamber should be able to measure not only the transverse momenta and position, but also the longitudinal position of the tracks $(z)$, with a resolution of $\sim 1 \mathrm{~mm}$. Good $z$ resolution also aids in matching DCH and SVT tracks and in projecting tracks to the DIRC and the calorimeter.

For low-momentum particles, the DCH provides PID by measurement of the $\mathrm{d} E / \mathrm{d} x$, thus allowing the $\mathrm{K} / \pi$ separation up to $\sim 700 \mathrm{MeV} / c$. This capability is complementary to that of the DIRC in the barrel region, while in the extreme backward and forward directions, the $\mathrm{DCH}$ is the only device to discriminate between different particle hypotheses.

\subsubsection{Detector Layout}

The design adopted for the DCH is illustrated in Fig. 2.10. It consists of a $280 \mathrm{~cm}$ long cylinder located within the volume inside the DIRC and outside the PEP-II support tube [39]. The active volume provides charged particle tracking over the polar angle range $17.2^{\circ}<\theta_{L A B}<152.6^{\circ}$. 


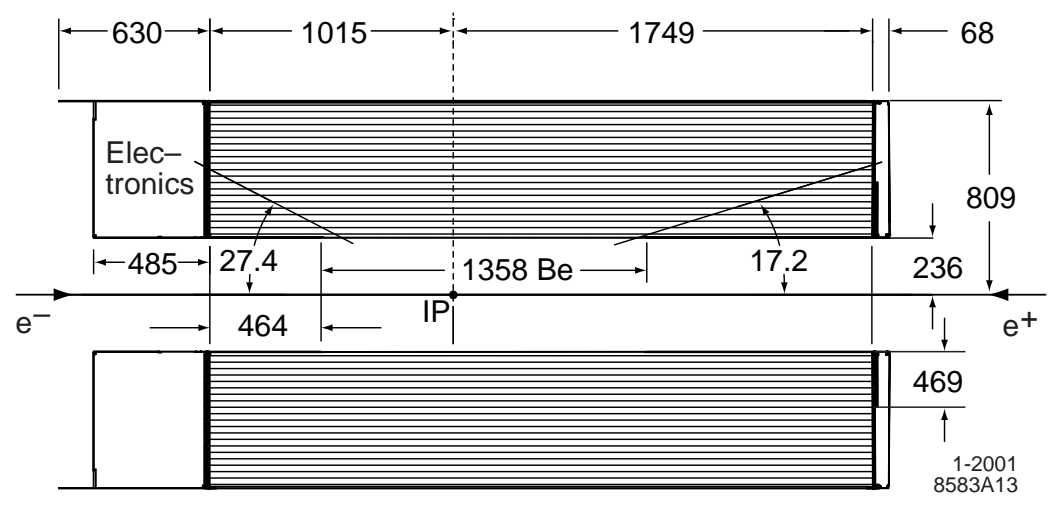

Figure 2.10: Longitudinal section of the drift chamber. Lengths are in $\mathrm{mm}$, angles in degrees.

The drift system consists of 7104 hexagonal cells, approximately $1.8 \mathrm{~cm}$ wide by $1.2 \mathrm{~cm}$ high, arranged in 40 concentric layers. Each hexagonal cell consists of one sense wire surrounded by six field-shaping wires, as shown in Fig. 2.11. In such a configuration, an approximately circular symmetry of equipotential contours is reached over a large portion of the cell. A positive high voltage is applied to the sense wires, while the field wires are at ground potential.

The 40 concentric layers are grouped by 4 into super layers, as shown in Fig. 2.11 for the four innermost super-layers. This arrangement enables local segment finding and leftright ambiguity resolution, even if one out of four signals is missing. For this reason two different wire types are used: the axial type wires (A), parallel to the $z$-axis, provide position measurements in the $x-y$ plane, while longitudinal position information is obtained with wires placed at small angles with respect to the $z$-axis (stereo wires, $\mathrm{U}$ or $\mathrm{V}$ type). Sense and field wires have the same orientation in each super-layer and are alternating following the scheme AUVAUVAUVA, as shown in Fig. 2.11.

The 40 layers provide up to 40 spatial and ionization loss measurements for charged particles with $p_{T}$ greater than $180 \mathrm{MeV} / c$. In order to reduce the impact of multiple scattering on $p_{T}$ resolution, material within the chamber volume has been minimized $\left(0.2 \% X_{0}\right)$ using low-mass aluminum field-wires and a helium-isobutane gas mixture.

The inner cylindrical wall of the DCH is kept thin to facilitate the matching of the SVT and DCH tracks, to improve the track resolution for high momentum tracks, and minimize the background from photon conversion and interaction. In addition, the high voltage (HV) distribution and all the readout electronics are mounted on the backward endplate of the chamber, in order to minimize the material in the forward direction, so as not to degrade the DIRC and EMC performance. 


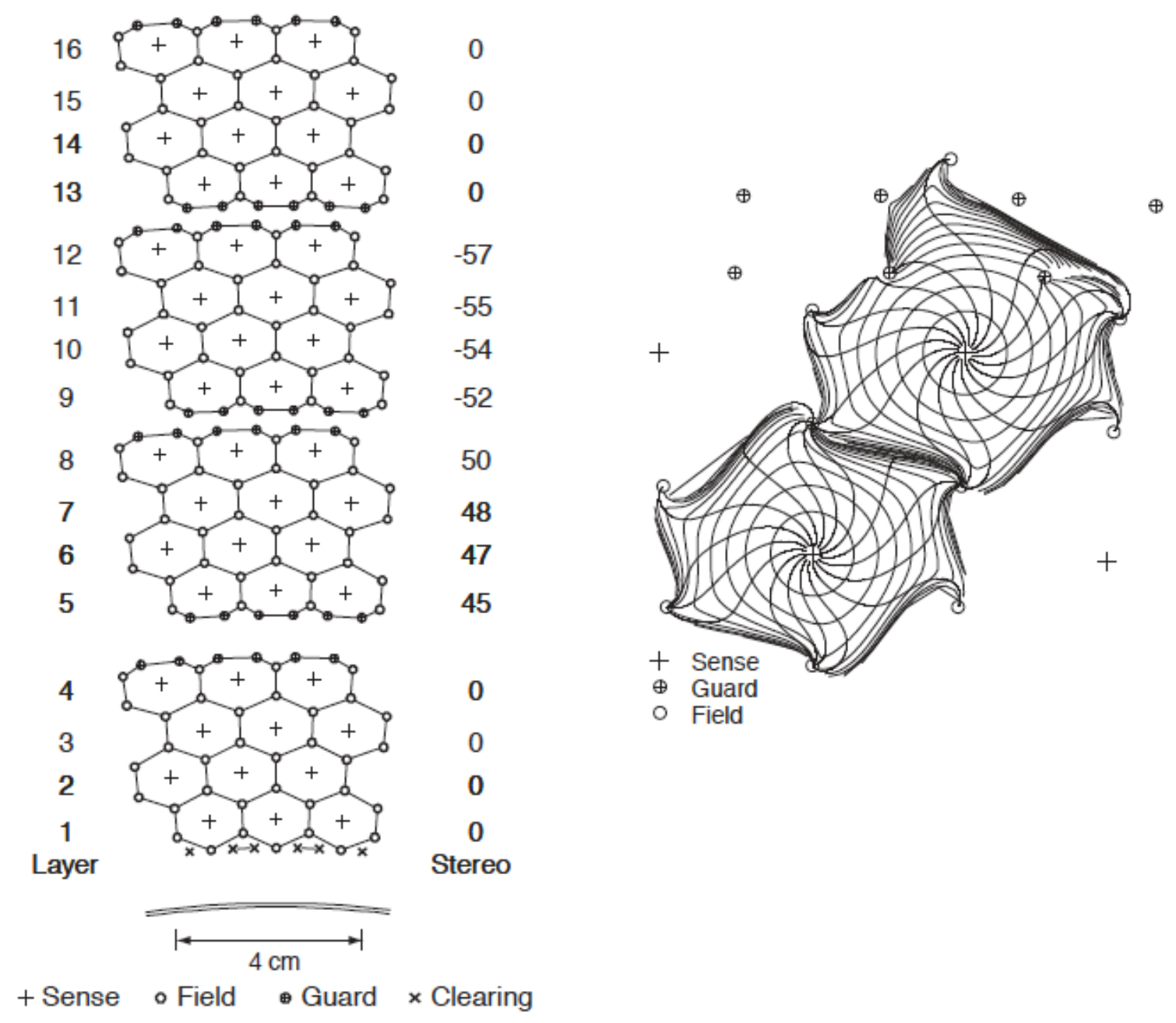

Figure 2.11: BABAR DCH cell configuration. In the left plot, lines have been added between field wires to aid in visualization of the cells, and the number on its right side give the stereo angles (mrad) of sense wires in each layer. The $1 \mathrm{~mm}$-thick beryllium inner cylinder is also shown inside the first layer. In the right plot, the cell structure is shown. The plus sign, open circles, filled circles, and crosses denote sense wire, field wires, guard wires and clearing wires, respectively.

\subsubsection{Detector Performance}

\section{Tracking Efficiency and Resolution}

The drift chamber reconstruction efficiency has been measured on data in selected samples of multi-hadron events by exploiting the fact that the tracks can be reconstructed independently in the SVT and in the DCH. The absolute DCH tracking efficiency is determined as the fraction of all the tracks detected in the SVT which are also reconstructed by the DCH when they fall within its acceptance. In Fig. 2.12 [33] the dependency on the transverse momentum and polar angle is shown. At the design voltage of $1960 \mathrm{~V}$ the DCH efficiency averages to $(98 \pm 1) \%$ for tracks above $200 \mathrm{MeV} / c$ and polar angle $\theta>500$ 
$\operatorname{mrad}\left(29^{\circ}\right)$. At the typical operating voltage of $1930 \mathrm{~V}$ it decreases by about $2 \%$.

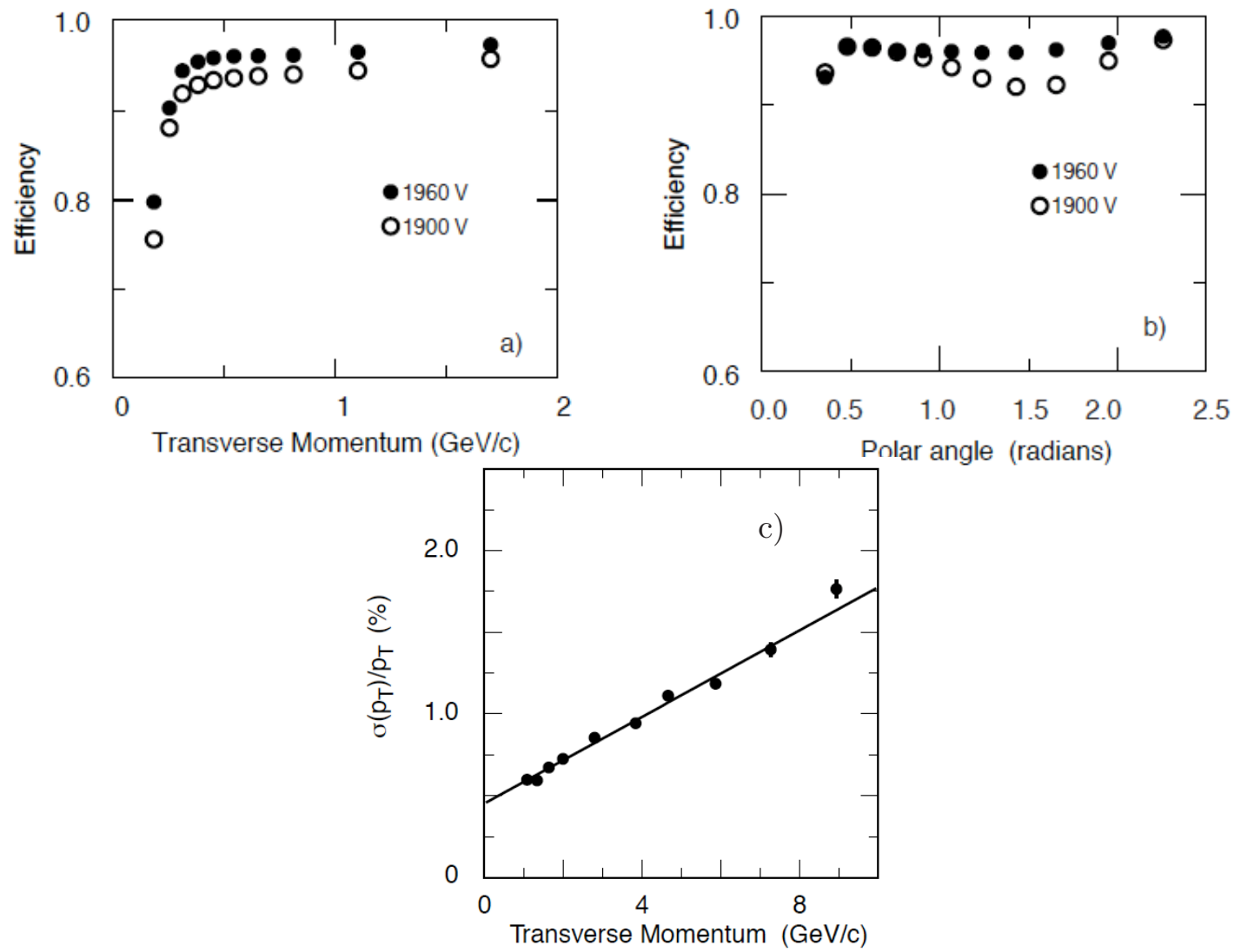

Figure 2.12: Track reconstruction efficiency in the DCH at operating voltages of $1900 \mathrm{~V}$ and $1960 \mathrm{~V}$, as a function of (a) transverse momentum, and (b) polar angle. In plot c) the $p_{T}$ resolution determined from cosmic ray muons is shown.

The $p_{T}$ resolution, directly related to the curvature resolution, is measured as a function of $p_{T}$ in cosmic ray studies (see Fig. 2.12c). The data are well represented by a linear function:

$$
\frac{\sigma p_{T}}{p_{T}}=(0.13 \pm 0.01) \% \cdot p_{T}+(0.45 \pm 0.03) \%
$$

where $p_{T}$ is measured in $\mathrm{GeV} / c$. The first contribution (dominating at high transverse momentum) comes from the curvature error due to the finite spatial measurement resolution. The second term (dominating at low momenta) is due to multiple Coulomb scattering.

\section{$\mathrm{d} E / \mathrm{d} x$ Resolution}

The specific energy loss, $\mathrm{d} E / \mathrm{d} x$, for charged particles traversing the $\mathrm{DCH}$ is derived from measurement of the total charge deposited in each drift cell. It is computed as a truncated mean from the lowest $80 \%$ of the individual $\mathrm{d} E / \mathrm{d} x$ measurements. Various corrections are applied to remove sources of bias (such as changes in the gas gain due to temperature and pressure variations, differences in cell geometry) that would degrade the accuracy of the primary ionization measurement. 
The left plot (a) of Fig. 2.13 shows the distribution of the reconstructed and corrected $\mathrm{d} E / \mathrm{d} x$ from the $\mathrm{DCH}$ as a function of the track momenta. The superimposed BetheBloch prediction for different masses have been determined using various particle control samples. The achieved resolution is typically 7.5\% (as shown in the right plot (b) of Fig. 2.13 for $e^{ \pm}$from Bhabha scattering), limited by the number of samples and Landau fluctuations. A $3 \sigma$ separation between kaons and pions can be achieved up to momenta of about $700 \mathrm{MeV} / c[40]$.
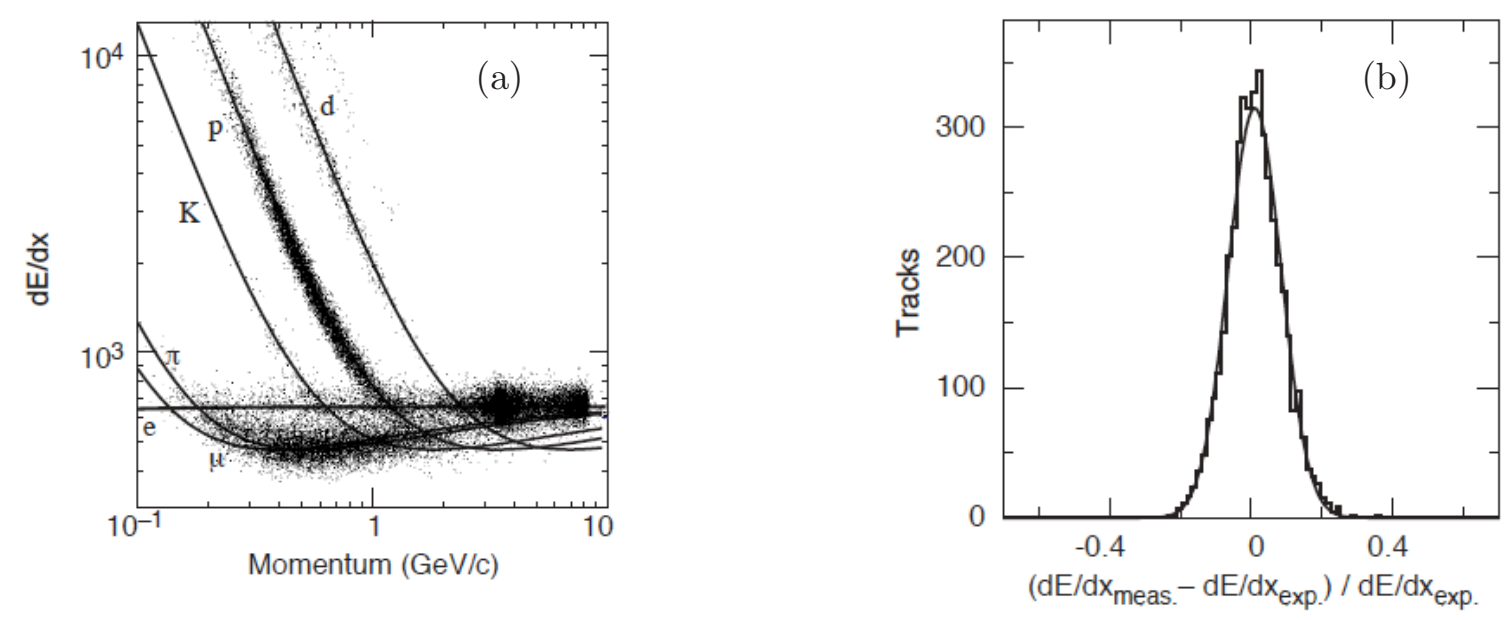

Figure 2.13: (a) $\mathrm{d} E / \mathrm{d} x$ as a function of track momentum. The data include large samples of beam background triggers, as evident from the high rate of protons. The unit of the $\mathrm{d} E / \mathrm{d} x$ curve is arbitrary due to the corrections for the deposited charge in individual DCH cells. (b) Difference between the measured and expected $\mathrm{d} E / \mathrm{d} x$ for $e^{ \pm}$from Bhabha scattering. The curve is the result of the fit to the data described in the text.

\subsection{The Cherenkov Light Detector}

The PID at low momenta exploits primarily the $\mathrm{d} E / \mathrm{d} x$ measurements in the DCH and SVT. For momenta above $700 \mathrm{MeV} / c$, the $\mathrm{d} E / \mathrm{d} x$ information does not allow one to separate pions and kaons and, therefore, a dedicated PID sub-detector is needed.

The Detector of Internally Reflecting Cherenkov Radiation (DIRC), has been designed to provide $K / \pi$ separation of $3 \sigma$, for all tracks with momenta from the pion Cherenkov threshold up to more than $4 \mathrm{GeV} / c$.

\subsubsection{Detector Layout}

The DIRC [41] is a novel type of ring-imaging Cherenkov detector, based on the principle that the magnitude of angles are maintained upon reflection from a flat surface.

Fig. 2.14 illustrates the principles of light production, transport, and imaging.

The radiator material of the DIRC is synthetic fused silica in the form of long, thin bars with a rectangular cross section. The bars, which are $17 \mathrm{~mm}$-thick, $35 \mathrm{~mm}$-wide, and 4.9 


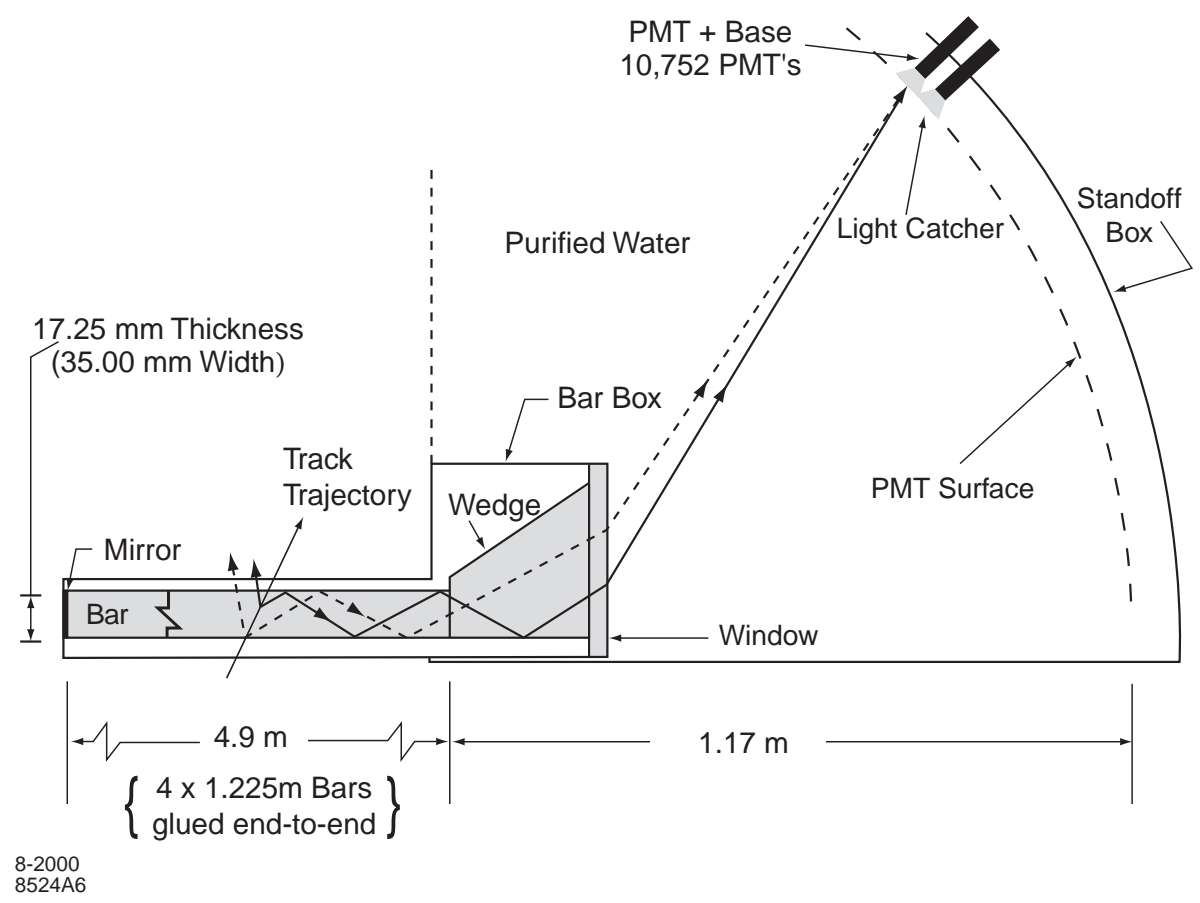

Figure 2.14: Schematic of the DIRC fused silica radiator bar and imaging region.

m-long, are placed into 12 hermetically sealed containers, called bar boxes, made of very thin aluminum-hexcel panels. Each bar box contains 12 bars, for a total of 144 bars.

The solid angle subtended by the radiator bars corresponds to $94 \%$ of the azimuth and $83 \%$ of the cosine of the polar angle in the CM system. The DIRC is the only subdetector not fully covering the forward region, with $25.5^{\circ}<\theta<141.4^{\circ}$. The total thickness of the DIRC material (bars and support structure) at normal incidence is only $8 \mathrm{~cm}$, corresponding to $17 \% X_{0}$. Such a thin Cherenkov detector allows a larger inner tracking volume, which is needed to achieve the desired momentum resolution, and a compact outer electromagnetic calorimeter with improved angular and energy resolution and limited costs.

The bars serve both as radiators and as light pipes for the portion of the light trapped in the radiator by total internal reflection, where the internal reflection coefficient of the bar surfaces is greater than 0.9992 per bounce.

A charged particle with velocity $v>c / n$, traversing the fused silica bar (refraction index $n=1.473)$, generates a cone of Cherenkov photons of half-angle $\theta_{c}$ with respect to the particle direction, where $\theta_{c}$ is the Cherenkov angle and $\cos \theta_{c}=1 / \beta n(\beta=v / c, v$ is the velocity of the particle). For particles with $\beta Z 1 / n$, photons will lie within the total internal reflection limit, and will be transported to either one or both ends of the bar, depending on the particle incident angle. To avoid instrumenting both ends of the bars with photon detectors, a mirror is placed at the forward end, perpendicular to the bar axis, to reflect incident photons to the backward, instrumented end.

Once photons arrive at the instrumented end, most of them emerge into a water-filled 
expansion region, called standoff box, containing 6000 liters of purified water $(n=1.346)$. A fused silica wedge at the exit of the bar reflect photons at large angles relative to the bar axis, reducing the size of the required detection surface. The photons are detected by an array of densely packed photon-multiplayer tubes (PMTs), each surrounded by reflecting "light catcher" cones to capture light that would otherwise miss the active area of the PMT. The PMTs are placed at a distance of about $1.2 \mathrm{~m}$ from the bar end. The expected Cherenkov light pattern at this surface is essentially a conic section, where the opening angle is the Cherenkov production angle modified by refraction at the exit from the fused silica window.

\subsubsection{Detector Performance}

In the absence of correlated systematic errors, the resolution $\left(\sigma_{C, \text { track }}\right)$ on the track Cherenkov angle should scale as

$$
\sigma_{C, \text { track }}=\frac{\sigma_{C, \gamma}}{\sqrt{N_{\gamma}}}
$$

where $\sigma_{C, \gamma}$ is the single photon Cherenkov angle resolution, and $N_{\gamma}$ is the number of detected photons.

The single photon Cherenkov resolution has been measured to be $10.2 \mathrm{mrad}$ in di-muon events (see Fig. 2.15(a) [33]). The main contributions to it come from the geometry of the detector (the size of the bars, the diameter of the PMTs, and the distance between the bars and the PMTs give a 7 mrad contribution) and from the spread of the photon production angle, dominated by a $5.4 \mathrm{mrad}$ chromatic term. The measured time resolution (Fig. 2.15(b)) is $1.7 \mathrm{~ns}$, close to the intrinsic $1.5 \mathrm{~ns}$ transit time spread of the PMTs.

In Fig. 2.16 the number of detected photons is shown as a function of the polar angle. It increases from a minimum of about 16 at the center of the barrel $\left(\theta \approx 90^{\circ}\right)$ to well over 50 at large polar angles (in the forward and backward directions), corresponding to the fact that the path-length in the radiator is longer for tracks emitted at large dip angles (greater number of Cherenkov photons produced in the bars) and the fraction of photons trapped by total internal reflection rises. This feature is very useful in the BABAR environment, where the particles are emitted preferentially in the forward direction as a consequence of the boost of the CM. The bump at $\cos \theta=0$ is a result of the fact that for tracks at small angles internal reflection of the Cherenkov photons occurs in both the forward and backward directions. The small decrease of the number of photons from the backward direction to the forward one is a consequence of the photon absorption along the bar before reaching the stand-off box in the backward end.

The combination of the single photon Cherenkov angle resolution, the distribution of the number of detected photons versus the polar angle, and the polar angle distribution of the charged tracks yield a typical track Cherenkov angle resolution which is about $2.5 \mathrm{mrad}$ 

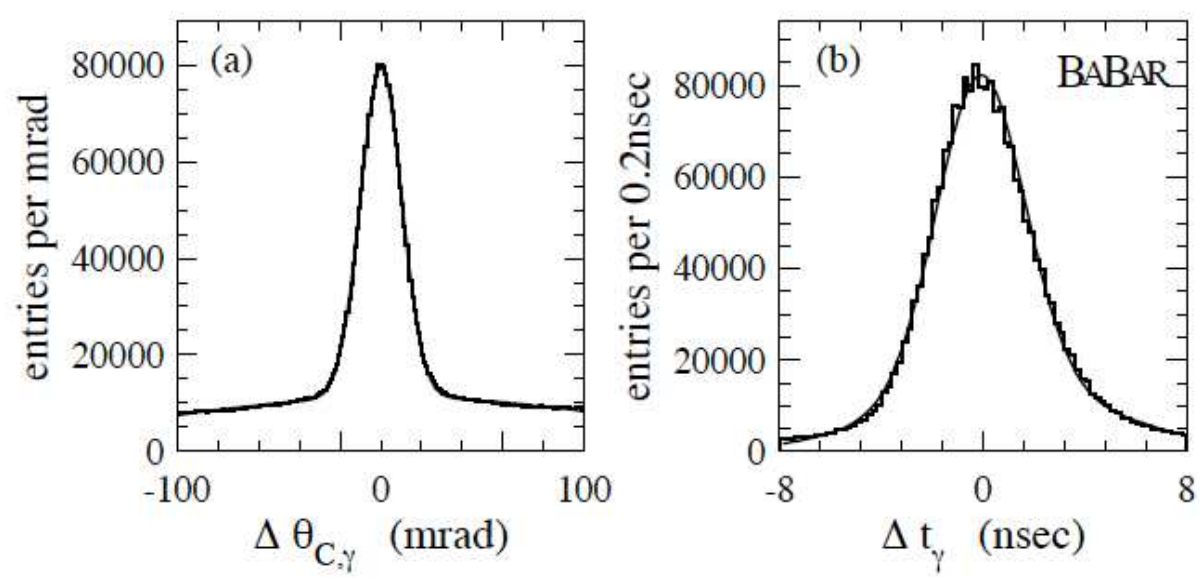

Figure 2.15: The difference between the measured and expected values of the Cherenkov angle for single photons, $\Delta \theta_{C, \gamma}$ (a), and the measured and expected photon arrival time, for single muons in $\mu^{+} \mu^{-}$events (b).

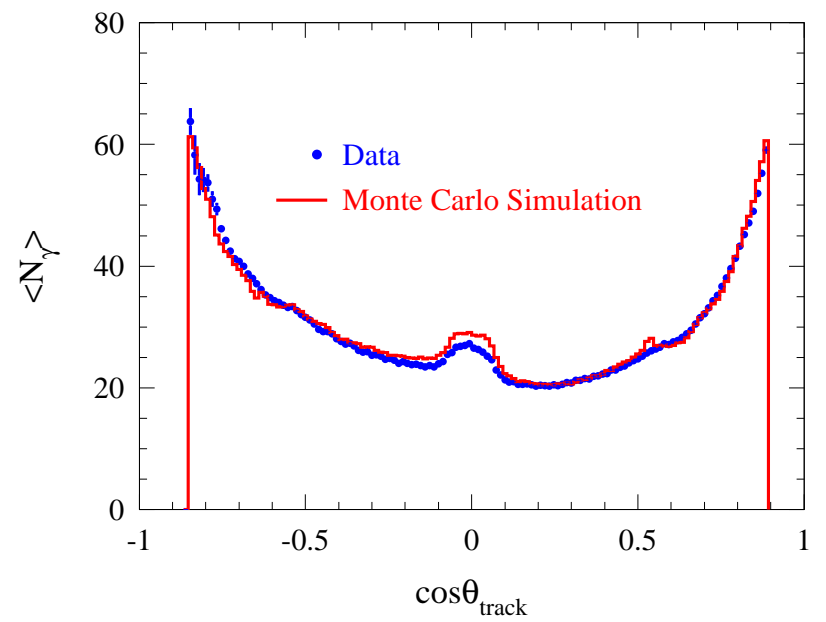

Figure 2.16: Number of detected photons versus track polar angle for reconstructed tracks in di-muon events compared to the Monte Carlo simulation.

for muons in di-muon events.

The efficiency for correctly identifying a charged kaon that traverses a radiator bar, and the probability to wrongly identify a pion as a kaon, are determined using $D^{* \pm} \rightarrow D^{0} \pi^{ \pm}\left(D^{0} \rightarrow\right.$ $K^{\mp} \pi^{ \pm}$) decays reconstructed in data, where $K^{\mp} / \pi^{ \pm}$tracks are identified through the charge correlation with the $\pi^{ \pm}$from the $D^{* \pm}$ decay.

The distribution of the Cherenkov angle for pions and kaons as a function of the momentum is shown in Fig. 2.17(a), while Fig. 2.17(b) reports the separation between kaons and pions, which is about 4.3 standard deviations at $3 \mathrm{GeV} / c$.

The DIRC is intrinsically a three-dimensional imaging device, using position and arrival time of the PMT signals. In order to associate the photon signals with the track traversing a bar, the vector pointing from the center of the bar end to the center of each PMT is 

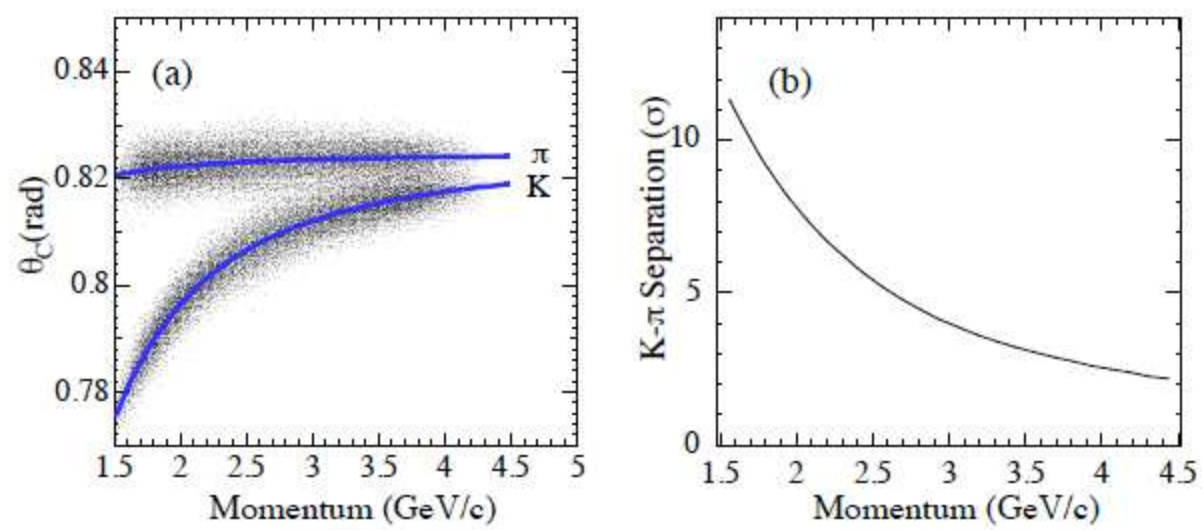

Figure 2.17: (a) The measured Cherenkov angle for pions (upper band) and kaons (lower band) from $D^{* \pm} \rightarrow D^{0} \pi^{ \pm}, D^{0} \rightarrow K^{\mp} \pi^{ \pm}$decays reconstructed in data. The curves show the expected angle $\theta_{C}$ as a function of LAB momentum, for $K$ and $\pi$ mass hypotheses. (b) The average difference between the expected value of $\theta_{C}$ for pions and kaons divided by the uncertainties $\left(\left|\theta_{C}^{K}-\theta_{C}^{\pi}\right| / \sigma_{\theta_{C}}\right)$, as a function of momentum.

taken as a measurement of the photon propagation angles $\left(\alpha_{x}, \alpha_{y}, \alpha_{z}\right)$. As the track position and angles are known from the tracking system, these three angles can be used to determine the two Cherenkov angles $\left(\theta_{C}, \phi_{C}\right)$. This constraint on $\theta_{C}$ and $\phi_{C}$ is particularly useful in suppressing hits from beam-generated background and from other tracks in the same event and also in resolving some ambiguities in the association between the PMT hits and the track (for instances, the forward-backward ambiguity between photons that have or have not been reflected by the mirror at the forward end of the bars).

The observable used to distinguish between signal and background photons is the difference between the measured and the expected photon time $\left(\Delta t_{\gamma}\right.$, shown in Fig. 2.15). The expected photon arrival time is calculated for each photon using the track time-of-flight assuming it to be a charged pion, and the photon propagation time within the bar, the wedge, and the water filled standoff box. The measured photon arrival time is obtained from the recorded time of the candidate signal in the PMT, after calibration. The effect of applying the PMT time information in a di-muon event [33] is shown in Fig. 2.18. The background hits, principally due to the low energy photons from the accelerator, are reduced by about a factor 40 . 


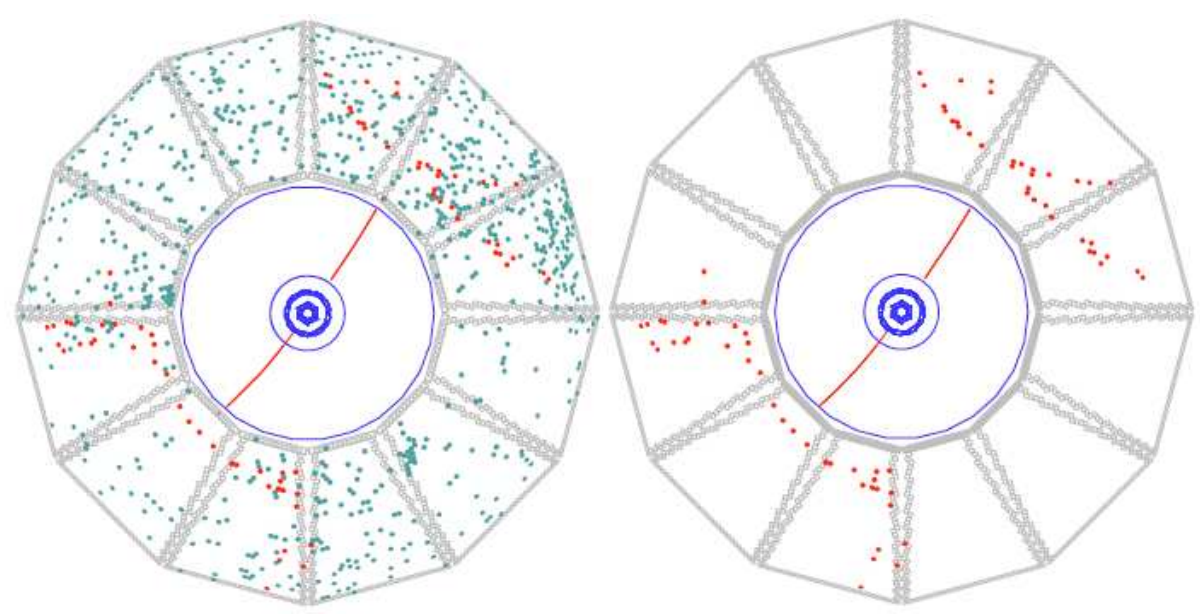

Figure 2.18: Display of an $e^{+} e^{-} \rightarrow \mu^{+} \mu^{-}$event reconstructed with two different time cuts. On the left, all DIRC PMTs with signal within $300 \mathrm{~ns}$ of the trigger time window are shown. On the right, only DIRC PMTs with signal within 8 ns of the expected Cherenkov photon arrival time are displayed.

\subsection{The ElectroMagnetic Calorimeter (EMC)}

The BABAR electromagnetic calorimeter (EMC) is designed to measure electromagnetic showers with high efficiency, and excellent energy and angular resolution over an energy range between $20 \mathrm{MeV}$ (low photons from $\pi^{0}$ mesons from $B$-meson decays) and $9 \mathrm{GeV}$ (electrons from Bhabha scattering). It is also the primary sub-detector providing electronhadron separation.

Energy deposit clusters in the EMC with a lateral shape consistent with the expected pattern from an electromagnetic shower are identified as photons if they are not associated with any charged tracks extrapolated from the tracking devices (DCH and SVT). Otherwise, they are identified as electrons if they are matched to a charged track and the ratio between the energy $E$ measured in the EMC and the momentum $p$ measured by the tracking system is $E / p \approx 1$.

The measurement of extremely rare decays of $B$ mesons containing $\pi^{0}$ s (e.g., $B^{0} \rightarrow \pi^{0} \pi^{0}$ ) poses the most stringent requirements on energy resolution, namely of order $1-2 \%$. The $\pi^{0}$ mass resolution is dominated by the energy resolution for $\pi^{0}$ of energy less than $2 \mathrm{GeV}$, and by the angular resolution at higher energies. Therefore, the angular resolution is required to be a few mrad in order to maintain good $m_{\pi}^{0}$ resolution at all energies $\left(\sigma_{m_{\pi}^{0}} \sim 6.5 \mathrm{MeV}\right)$.

In addition, excellent photon identification at low energy $(\sim 20 \mathrm{MeV})$ is required for efficient reconstruction of decays containing multiple $\pi^{0}$ and $\eta$. Similar precision is required for efficient separation of electrons and hadrons, with purity required at the $0.1 \%$ level for momentum as low as $500 \mathrm{MeV} / \mathrm{c}$.

The need for high efficiency requires hermetic coverage of the acceptance region, while excellent resolution is achieved by minimizing the material in front of, and between, the 
active detector elements.

\subsubsection{Detector Layout}

The BABAR EMC [42] contains a cylindrical barrel and a conical endcap containing a total of 6580 CsI crystals doped with thallium (Tl) at $1 \%$ level. The main proprieties of CsI(Tl) are summarized in Tab. 2.4. The high light yield and the the small Molière radius give the excellent energy and angular resolution required, while the short radiation length allows shower containment at the BABAR energies with a relatively compact design. Furthermore, the high light yield and the emission spectrum permit efficient use of a silicon photodiodes readout. The transverse size of the crystals is chosen to be comparable to the Moliére radius in order to achieve the required angular resolution at low energies. This choice is the best compromise, since the electromagnetic shower has a natural lateral spread of the Moliére radius and the energy resolution would degrade if the transverse crystal size were chosen smaller than this radius, due to the summing of the electronic noise from several crystals.

\begin{tabular}{ll}
\hline \hline Parameter & Values \\
\hline Radiation Length & $1.85 \mathrm{~cm}$ \\
Molière Radius & $3.8 \mathrm{~cm}$ \\
Density & $4.53 \mathrm{~g} / \mathrm{cm}^{3}$ \\
Light Yield & $50000 \mathrm{\gamma} / \mathrm{MeV}$ \\
Light Yield Temp. Coeff. & $0.28 \% /{ }^{\circ} \mathrm{C}$ \\
Peak Emission $\lambda_{\max }$ & $565 \mathrm{~nm}$ \\
Refractive Index $\left(\lambda_{\max }\right)$ & 1.80 \\
Signal Decay Time & $680 \mathrm{~ns}(64 \%)$ \\
& $3.3 \mu \mathrm{s}(36 \%)$ \\
\hline \hline
\end{tabular}

Table 2.4: Proprieties of $\mathrm{CsI}(\mathrm{Tl})$.

Each crystal is a truncated trapezoidal pyramid, whose length increases from $29.6 \mathrm{~cm}$ (16 $\left.X_{0}\right)$ in the backward to $32.4 \mathrm{~cm}\left(17.5 X_{0}\right)$ in the forward direction to limit the effects of shower leakage from increasingly higher energy particles (see Fig. 2.19a). To minimize the material in front of the calorimeter, the support structure of the crystals (made in carbon fiber) and the front-end electronics are located at the outer radius of the EMC. To recover the small fraction of light that is not internally reflected, each crystal is wrapped with a white diffuse reflector (TYVEK), $25 \mu \mathrm{m}$ aluminum foil and $13 \mu \mathrm{m}$ Mylar foil for insulation. The scintillation light generated inside each crystal is detected by two independent silicon PIN diodes.

The barrel contains 5760 crystals arranged in 48 distinct rows containing 120 identical crystals, with an inner radius of $90 \mathrm{~cm}$, as shown in Fig. 2.19b. The forward end is closed by a separable endcap holding nine additional rows (1080 crystals). This geometry provides full azimuthal coverage, while the polar angle coverage is $15.8^{\circ}<\theta_{L A B}<140.8^{\circ}$. 

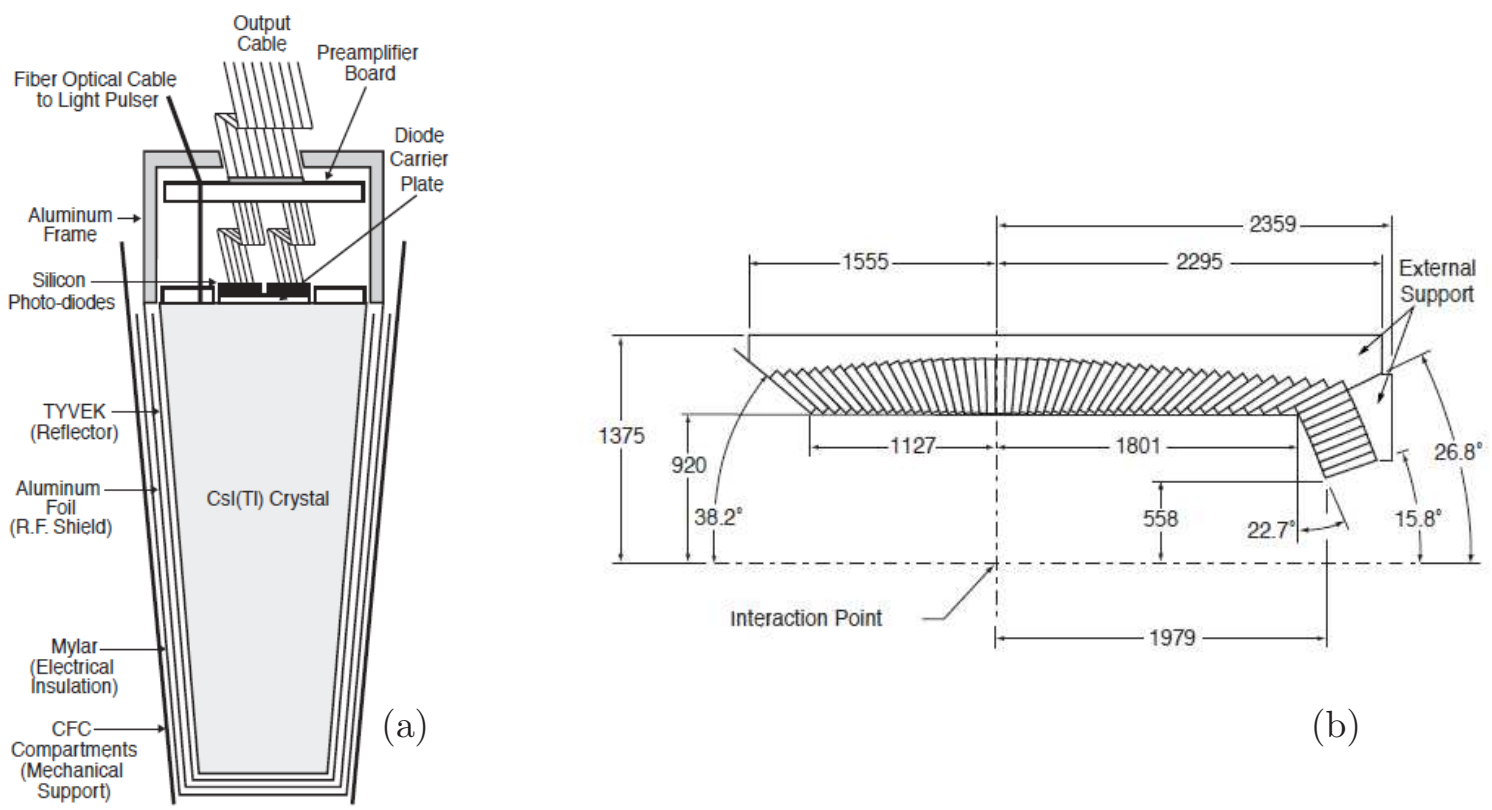

(b)

Figure 2.19: (a) Schematic view of one EMC crystal module. (b) Longitudinal cross section of the EMC (only the top half is shown). The detector is axially symmetric around the $z$-axis. All dimensions are given in $\mathrm{mm}$.

\subsubsection{Detector Performance}

The reconstruction of energy deposits in the calorimeter, is based on the concept of EMC cluster: it is defined as a contiguous array of crystals, all with energy above $0.5 \mathrm{MeV}$, whose total energy exceeds the threshold of $20 \mathrm{MeV}$ in order to suppress background processes.

\section{Energy Resolution}

The energy resolution of a homogeneous calorimeter is determined by fluctuations in the electromagnetic shower propagation and, for the BABAR EMC detector, is empirically described as a quadratic sum of a stochastic term $\sigma_{1}$ and a constant term $\sigma_{2}$ :

$$
\frac{\sigma_{E}}{E}=\frac{\sigma_{1}}{4 \sqrt{E(\mathrm{GeV})}} \oplus \sigma_{2},
$$

where $E$ and $\sigma_{E}$ refer to the energy of a photon and to its RMS error.

The stochastic term $\sigma_{1} / 4 \sqrt{E}$, which is dominant at low energies, arises primarily from the fluctuation in photon statistics, but it also depends on electron noise of the photon detector and electronics. The constant term $\sigma_{2}$ is dominant at higher energies $(>1 \mathrm{GeV})$. It arises from non uniformity in light collection, leakage or absorption in the material between and in front of the crystals, and uncertainties in the calibrations.

In $B A B A R$, the energy resolution of the EMC is measured on data selected control samples, 
including electrons and positrons from Bhabha scattering (energies between 3 and $9 \mathrm{GeV}$ ), photons from $\pi^{0}$ and $\eta$ decays (energies below $2 \mathrm{GeV}$ ), and from the decay $\chi_{c 1} \rightarrow J \phi \gamma$ $(E \sim 500 \mathrm{MeV})$. At low energies the resolution is determined through weekly calibrations performed with a radioactive source $\left({ }^{16} \mathrm{O}^{*}\right)$ of $6.13 \mathrm{MeV}$ photons.

A fit to the resolution dependence on the energy with the empirical parametrization of Eq. (2.4), shown in Fig. 2.20(a), yields:

$$
\frac{\sigma_{E}}{E}=\frac{(2.32 \pm 0.30) \%}{\sqrt[4]{E(\mathrm{GeV})}} \oplus(1.85 \pm 0.12) \%
$$

which is in reasonable agreement with the Monte Carlo studies of the expected resolution.
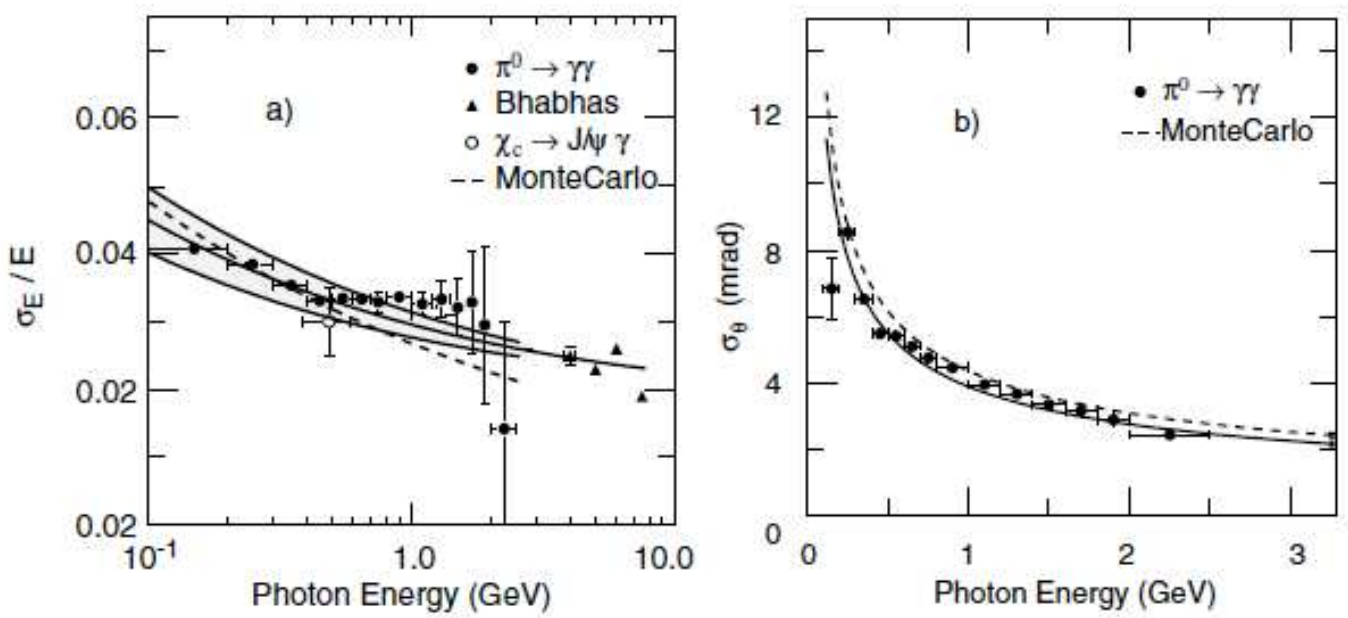

Figure 2.20: (a) Energy resolution for the EMC measured for photons and electrons from various processes. The solid curve is a fit to Eqn. (2.4) and the shaded area denotes the one sigma error of the fit. (b) EMC angular resolution measured using photon candidates from $\pi^{0}$ decays. The solid curve is a fit to Eqn. (2.6).

\section{Angular Resolution}

The angular resolution is determined by the transverse crystal size and the distance from the interaction point, and improves as the transverse size of the crystal decreases.

The measurement of the angular resolution is based on the analysis of $\pi^{0}$ and $\eta$ decays to two photons of approximately equal energy. The result is reported in Fig. 2.20. The resolution varies between about $12 \mathrm{mrad}$ at low energies and $3 \mathrm{mrad}$ at high energies. The data fits the empirical parameterization:

$$
\sigma_{\theta}=\sigma_{\phi}=\left(\frac{3.87 \pm 0.07}{\sqrt{E(\mathrm{GeV})}}+(0.00 \pm 0.04)\right) \operatorname{mrad}
$$




\section{Electron-Hadron Separation}

Electrons are separated from charged hadrons primarily on the basis of the shower energy, lateral shower moments, and track momentum. The most important variable for the discrimination of hadrons is the ratio of the shower energy to the track momentum $(E / p)$. Fig. 2.21 shows the efficiency for electron identification and the pion misidentification probability as a function of the track momentum and polar angle. The electron efficiency is measured using electrons from radiative Bhabha and $e^{+} e^{-} \rightarrow e^{+} e^{-} e^{+} e^{-}$events. The pion misidentification probability is measured using charged pions from $K_{S}^{0}$ decays and three-prong $\tau$ decay. For momenta above $1 \mathrm{GeV} / c$ the electron identification is about $91 \%$ with an average pion misidentification of $0.2 \%$.
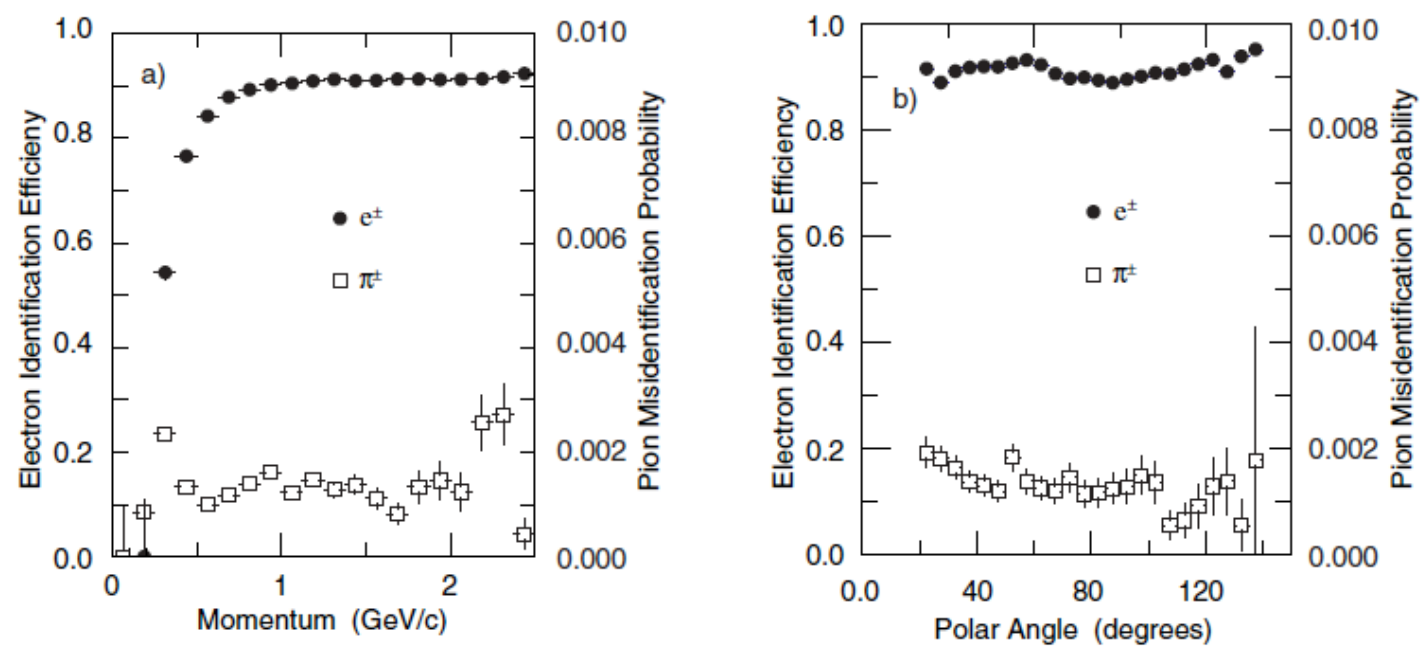

Figure 2.21: Electron efficiency and pion misidentification probability as a function of a) the particle momentum and b) the polar angle, measured in the laboratory system.

\subsection{The Instrumented Flux Return (IFR)}

The Instrumented Flux Return (IFR) is designed to identify muons and detect neutral hadrons (primarily $K_{L}^{0}$ ) over a wide range of momenta and angles. The principal requirements for the IFR are large solid angle coverage, good efficiency, and high background rejection for muons down to momenta below $1 \mathrm{GeV} / c$. For neutral hadrons, high efficiency and good angular resolution are crucial.

\subsubsection{Detector Layout}

The IFR uses the steel flux return of the magnet as a muon filter and hadron absorber. Single gap Resistive Plate Chambers [43] (RPCs) with two-coordinate readout have been initially chosen as detector. RPCs detect streamers from ionizing particles via capacitive readout strips. They offer the advantage of simple, low cost construction and the possibility 
of covering odd shapes with minimal dead space. Further benefits are large signals and fast response allowing for simple and robust front end electronics and good time resolution, typically 1-2 ns. The position resolution, of the order of few $\mathrm{mm}$, depends primarily on the segmentation of the readout strips. A cross section of an RPC is shown schematically in Fig. 2.22 .

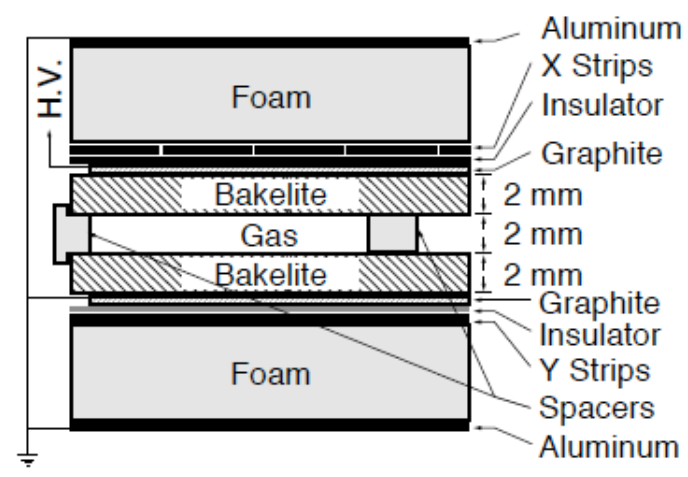

Figure 2.22: Cross section of a planar RPC with the schematics of the HV connection.

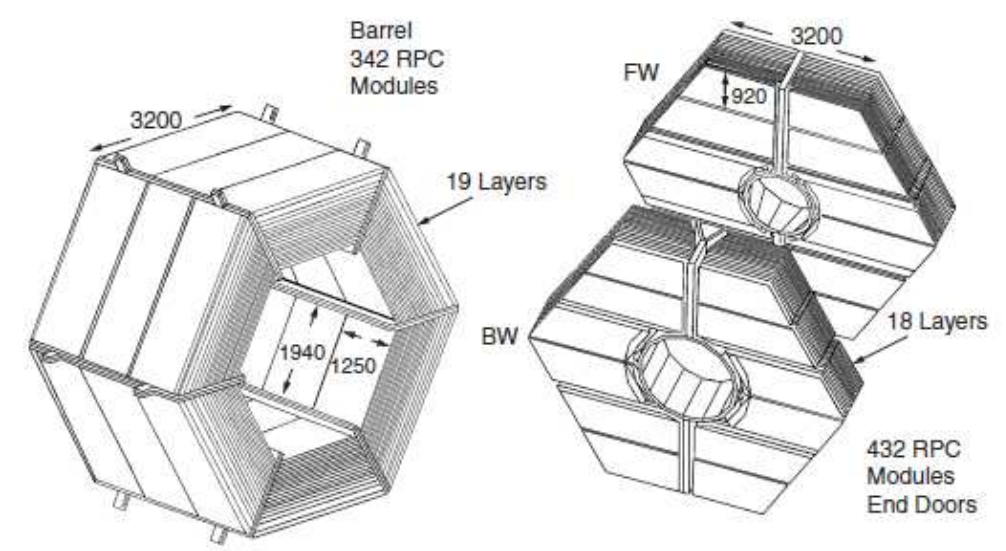

Figure 2.23: Overview of the IFR. On the left, the barrel sectors are shown and, on the right, the forward and backward end doors. The shape of the RPC modules and their dimensions are indicated.

The planar RPC consists of two $2 \mathrm{~mm}$-thick bakelite sheets, separated by a gap of $2 \mathrm{~mm}$. The bulk resistivity of the bakelite sheets has been especially tuned to $10^{11}-10^{12} \Omega \mathrm{cm}$, and the external surfaces are coated with graphite to achieve a surface resistivity of $\sim 100$ $\mathrm{k} \Omega /$ square. The two graphite surfaces are connected to high voltage $(\sim 8 \mathrm{kV})$ and protected by an insulating Mylar film. The bakelite surfaces facing the gap are treated with linseed oil.

Signals are read out capacitively on both sides of the gap, by external electrodes made of aluminum strips on a Mylar substrate.

The RPCs are installed in the gaps of the finely segmented steel of the barrel and the two end doors of the iron for the magnetic flux return [44], as illustrated in Fig. 2.23. The steel is segmented into 18 plates, increasing in thickness from $2 \mathrm{~cm}$ of the inner 9 plates to 10 $\mathrm{cm}$ of outermost plates, for a total of $65 \mathrm{~cm}$ (60 $\mathrm{cm}$ in the endcap), which corresponds to 
about 4 interaction lengths. The configuration has been optimized on the basis of Monte Carlo studies of muon penetration and charged and neutral hadron interaction.

Soon after the installation (Summer 1999), the efficiency of a large number of chambers (initially greater than $90 \%$ ) had started to deteriorate at a rate of $0.5-1 \%$ per month. In order to solve this loss of efficiency, an extensive improvement program has been developed with multiple solutions. The RPCs in the forward end-cap region have been replaced in Summer 2002 with new ones based on the same base concept but with improved fabrication technique. The RPCs in the barrel region have been replaced with Limited Streamer Tube (LST) detectors [45]. The research and design phase started in 2002 with the first installation phase in Summer 2004 and the second phase in Autumn 2006. In particular, in the first installation phase, the RPCs from the inner 18 layers of the top and bottom sextant were removed. In 12 of these layers LSTs were installed. In the remaining 6 layers of brass absorber were installed to increase the total interaction length and to compensate the loss in absorption material due to the inaccessibility, for mechanical reasons, of the last RPC layer. During the second installation phase (2006), the remaining 4 sextants of RPCs were replaced.

The base detector for a limited streamer tube [46] consist of a $100 \mu \mathrm{m}$ silver-plated wire, located at the center of a squared cell filled with gas. For the BABAR LSTs, the cell configuration is $17 \mathrm{~mm}$ wide, $15 \mathrm{~mm}$ high, and $3.8 \mathrm{~m}$ long. The anode wire is $100 \mu \mathrm{m}$ diameter gold-plated tungsten, and six wire holders are equally distributed over the length of a cell to prevent the wire from sagging and touching the cell walls, and to provide electrostatic stability. Three sides of the cell are painted with a water-based graphite paint and kept at ground potential.

A tube is made of a plastic extruded structure (see Fig. 2.24) consisting of 7 or 8 cells open on the top side and covered with a plastic plane. On the bottom side of this plane conductive strips are installed perpendicular to the wire direction. The extruded structure and the plane are inserted in plastic tubes, called sleeves, of matching dimensions for gas containment. Between the cell and the wire a high voltage is applied (a typical working point is $5.5 \mathrm{kV}$ ) and $\mathrm{HV}$ connectors are hosted on one endcap.

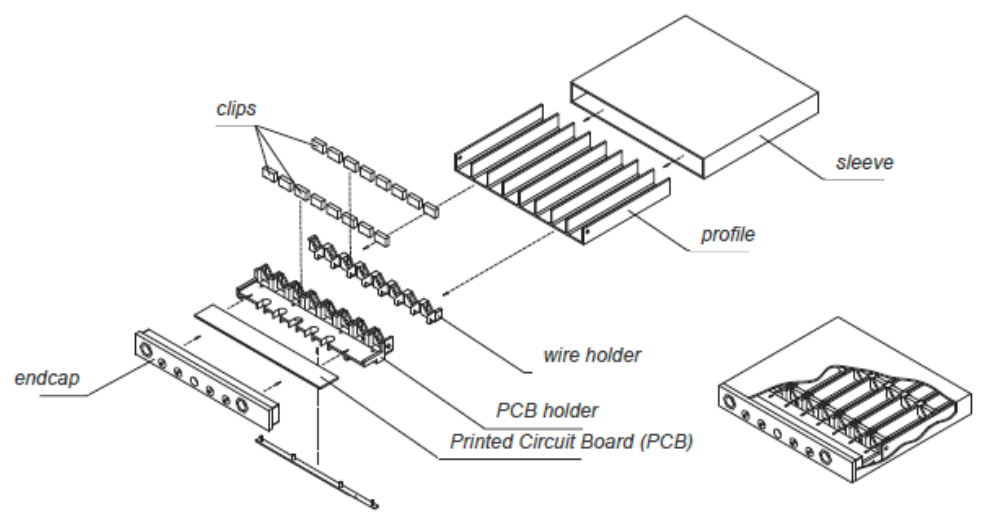

Figure 2.24: Schematic view of a standard Limited Streamer Tube configuration. 
If a charged particle passes through the cell, the gas is ionized and a streamer builds up, which can be readout from the wire. Simultaneously, a signal will be induced on the strip above. The charge on the wire is used for measuring the azimuthal coordinate $(\phi)$, and the induced charge on the strip for the $z$ coordinate, along the beam direction. Finally, the $r$ coordinate is taken from the layer position in the segmented steel, allowing a 3D information of the hit.

More than one year of studies was done before choosing the final LST design. In particular, several critical issues have been taken into consideration like selection of safe gas mixture, rate capability, wire surface quality and uniformity. Final results led to the configuration detailed above and a ternary gas mixture of $\mathrm{Ar} / \mathrm{C}_{4} \mathrm{H}_{1} 0 / \mathrm{CO}_{2}(3 / 8 / 89) \%$ was chosen.

\subsubsection{Detector Performance}

\section{Muon Efficiency}

The efficiency of RPCs and LSTs is evaluated using di-muon events collected both in normal condition data $\left(e^{+} e^{-} \rightarrow \mu^{+} \mu^{-}\right)$and monthly dedicated cosmic ray runs. The efficiency is found by counting the number of times a hit is found in a certain chamber when a charged track is expected to traverse it, based on information from the other tracking systems. The absolute efficiency at the nominal working voltage (typically 7.6 $\mathrm{kV}$ for RPC and 5.5 for LST) is stored in the BABAR condition database (see Sec. 2.9) for use in the event reconstruction software.

As previously said, soon after the installation, a progressive efficiency deterioration has been observed in a significant fraction of the RPC chambers, as shown in Fig. 2.25. Several tests were performed in order to understand the causes of the loss of efficiency, and it was found that a number of prototype RPCs developed similar efficiency problems after being operated above a temperature of $36^{\circ} \mathrm{C}$ for a period of two weeks ${ }^{3}$. In some of these modules evidence was found that the linseed oil had accumulated at various spots under the influence of the electric field [47].

After the installation of the LST detector, a stable muon efficiency was recovered, as shown in Fig. 2.26. The overall average efficiency at the end of the BABAR data-taking was about $88 \%$, slightly below the design efficiency. Beside the geometrical effect, the main sources of inefficiency are broken strips and wires which have been disconnected or kept to a lower voltage.

\footnotetext{
${ }^{3}$ Similar temperatures had been reached inside the iron during the first summer of operation due to the temperature in the experimental hall and the absence of a water cooling system.
} 


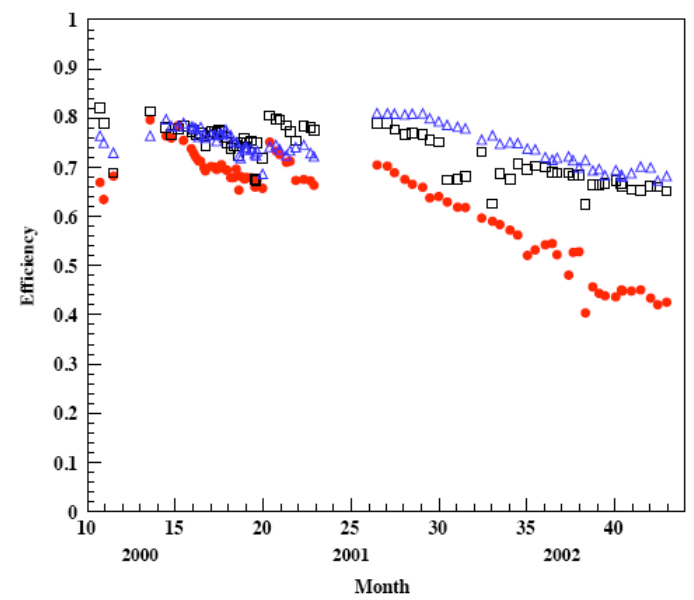

Figure 2.25: The average RPC efficiency in the barrel (red circles), forward end cap (blue triangles), and backward end cap (black squares) are shown as a function of time until summer 2002. The efficiency is evaluated using $\mu^{+} \mu^{-}$pairs from collision data.

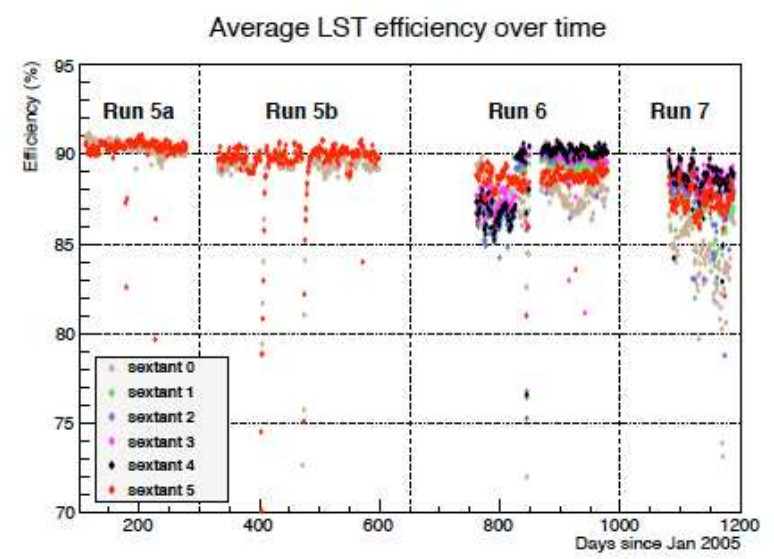

Figure 2.26: Time evolution of the average sextant detection efficiency of the LST detector. The inefficiency is localized in particular in the two innermost layers; for this reason it didn't affect the muon identification quality.

\section{Muon Identification}

While muon identification relies entirely on the IFR, other detector systems provide complementary information. Charged particles are reconstructed in the SVT and DCH and muon candidates are required to meet the criteria for minimum ionizing particles in the EMC. Charged tracks that are reconstructed in the tracking system are extrapolated to the IFR taking into account the non-uniform magnetic field, multiple scattering, and the average energy loss. The projected intersections of a track with the RPC and LST planes are computed and, for each readout plane, all clusters (groups of adjacent hits) detected within a predefined distance from the predicted intersection are associated with the track. Quantities used for $\pi / \mu$ discrimination, in addition to the penetration depth in the iron of the track, are the average number and r.m.s. of the distribution of the RPC and LST hits per layer. The hit multiplicity per layer is expected to be larger for pions, producing a hadronic interaction, than for muons. 
The performance of muon identification has been tested on samples of muons from $\mu \mu e e$ and $\mu \mu \gamma$ final state and pions from three-prong $\tau$ decays and $K_{S} \rightarrow \pi^{+} \pi^{-}$decays. The typical muon identification efficiency and the pion misidentification probability as a function of the track momentum and polar angle are shown in Fig. 2.27, while Fig. 2.28 displays the performance of a muon selector based on a neural network in the forward and barrel region, for different years of data taking. Due to the problems and replacement described above, the efficiency of the IFR detector shows large fluctuation through the years.
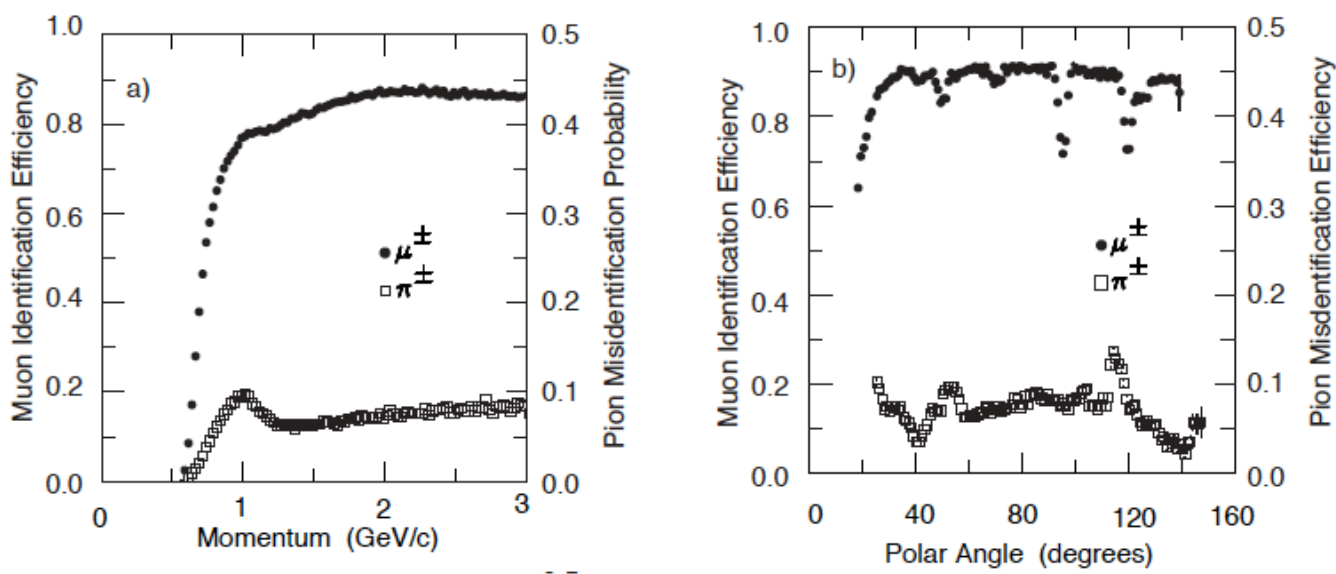

Figure 2.27: Muon efficiency (left scale) and pion misidentification probability (right scale) as a function of a) the laboratory track momentum, and b) the polar angle (for $1.5<<$ $3.0 \mathrm{GeV} / c$ momentum).
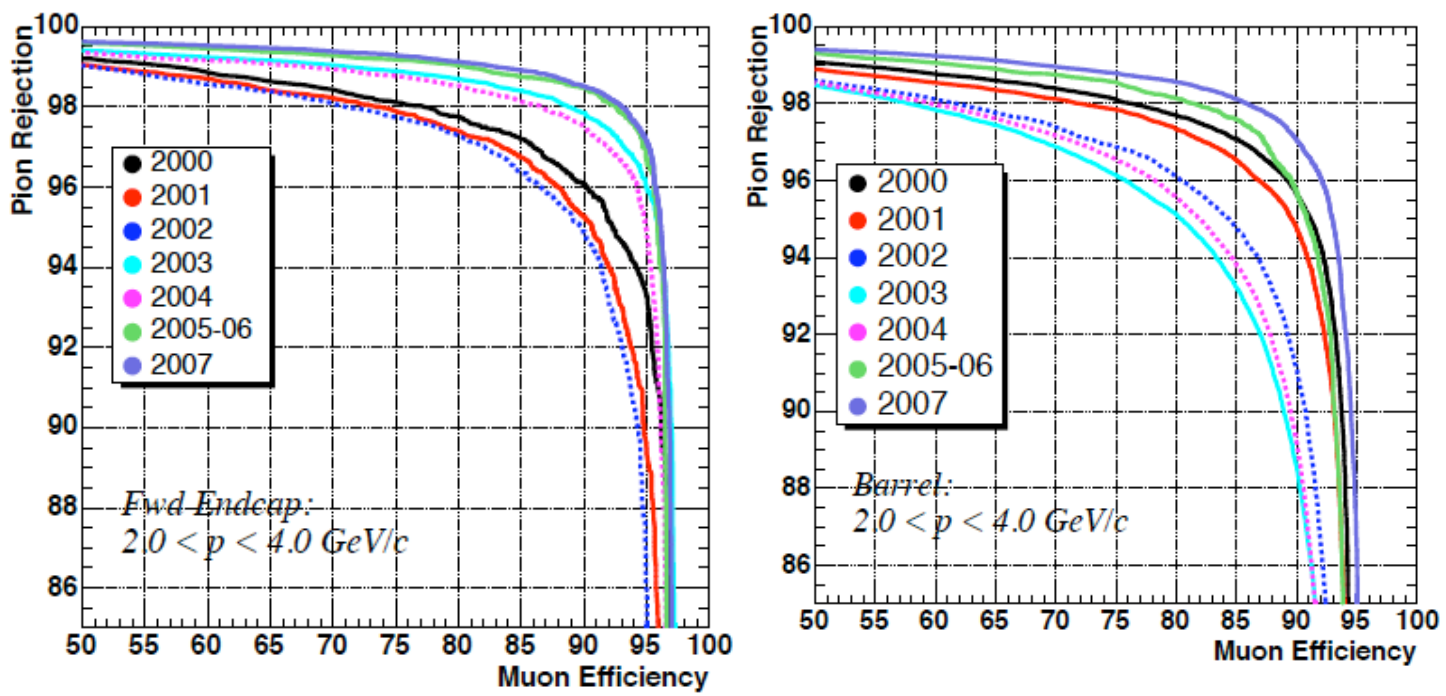

Figure 2.28: Pion misidentification vs. muon efficiency rate of neural network algorithm for different period of BABAR data taking.

\section{Neutral Hadron Detection}

Neutral hadrons interacting in the steel of the IFR are identified as clusters that are not associated with a charged track. Since a significant fraction of neutral hadrons interact 
before reaching the IFR, information from the EMC and the IFR is combined: neutral showers in the EMC are associated with the neutral hadrons detected in the IFR if their production angles, taken from the first interaction point in the detector, are consistent with each other. The $K_{L}^{0}$ detection efficiency and angular resolution are measured on a control sample of $K_{L}^{0}$ produced in $e^{+} e^{-} \rightarrow \phi \gamma \rightarrow K_{L}^{0} K_{S}^{0} \gamma$ processes, where the $K_{L}^{0}$ direction is inferred from the missing momentum calculated from the particles that are reconstructed in the final state $\left(\gamma\right.$ and $\left.K_{S}^{0}\right)$. The $K_{L}^{0}$ reconstruction efficiency increases roughly linearly with momentum between $20 \%$ at $1 \mathrm{GeV} / c$ and $40 \%$ at $4 \mathrm{GeV} / c(\mathrm{EMC}$ and IFR combined), and the angular resolution is of the order of $50 \mathrm{mrad}$.

\subsection{The BABAR Trigger}

The BABAR trigger is designed to select a large variety of physics processes rejecting background events and keeping a total event rate around $300 \mathrm{~Hz}$ so as not to overload the downstream processing. The trigger must select the physics events of interest with very high and/or well understood efficiency, depending on the particular mode.

The trigger system is implemented as a two-level hierarchy, a hardware based Level 1 (L1) followed by a software based Level $3(\mathrm{~L} 3)^{4}$. The goal of the L1 hardware trigger is to reduce the rate to a level acceptable for the L3 software trigger, which runs on a farm of commercial processors. The L1 trigger is optimized for simplicity and speed, and is designed to provide an output trigger rate of the order of $2 \mathrm{kHz}$ or less. The L1 trigger selection is based on charged tracks in the DCH, showers in the EMC, and tracks detected in the IFR. Its fixed response latency for a given collision is $11 \mu \mathrm{s}$, with a jitter of about $1 \mu \mathrm{s}$.

Based on both the complete event and L1 trigger information, the L3 software algorithms select events of interest allowing them to be transferred to mass storage for further analysis. Dedicated L1 trigger processors receive data which are continuously clocked in from the $\mathrm{DCH}, \mathrm{EMC}$, and IFR detector subsystem. The L1 trigger processor produces a $30 \mathrm{MHz}$ clocked output to the Fast Control and Timing System (FCTS) that can optimally mask or prescale input triggers. Tab. 2.5 summarizes the cross section, production rates and L1 trigger rates for the main physical processes at the $\Upsilon(4 S)$ resonance for the design luminosity $\mathcal{L}=3 \cdot 10^{33} \mathrm{~cm}^{-2} \mathrm{~s}^{-1}$.

The L3 trigger is implemented as a software-based trigger that makes use of the complete event information for taking its decision, including the output of the L1 trigger processors and of the FCTS. The selection decision is primarily taken by two set of orthogonal filters, one exclusively based on the DCH information, the other based on the EMC data only. The DCH filters select events containing at least one high $p_{T}$ track $\left(p_{T}>600 \mathrm{MeV} / c\right)$ or

\footnotetext{
${ }^{4}$ An intermediate Level 2 (L2) software trigger was originally foreseen in the very early stages of $B A B A R$ design, but it was soon merged in the L3 trigger.
} 


\begin{tabular}{cccc}
\hline \hline Event type & $\begin{array}{c}\text { Cross section } \\
\mathrm{nb}\end{array}$ & $\begin{array}{c}\text { Production Rate } \\
\mathrm{Hz}\end{array}$ & $\begin{array}{c}\text { L1 Trigger Rate } \\
\mathrm{Hz}\end{array}$ \\
\hline$b \bar{b}$ & 1.1 & 3.2 & 3.2 \\
other $q \bar{q}$ & 3.4 & 10.2 & 10.1 \\
$e^{+} e^{-}$ & $\sim 53$ & 159 & 156 \\
$\mu^{+} \mu^{-}$ & 1.2 & 3.5 & 3.1 \\
$\tau^{+} \tau^{-}$ & 0.9 & 2.8 & 2.4 \\
\hline \hline
\end{tabular}

Table 2.5: Effective cross section, production rates, and trigger rates for the principal physics processes at the $\Upsilon(4 S)$ for a luminosity of $\mathcal{L}=3 \cdot 10^{33} \mathrm{~cm}^{-2} \mathrm{~s}^{-1}$.

two low $p_{T}$ tracks, originating from the interaction point. The all-neutral trigger for L3 is based on information from the EMC. The EMC filter identifies energy clusters with a sensitivity sufficient for finding minimum ionizing particles. To filter out noise, individual crystal signals below an energy threshold $20 \mathrm{MeV}$ or which lie outside a $1.3 \mu$ s time window around the event are rejected. Clusters with a total energy above $100 \mathrm{MeV}$ are retained, and the energy weighted centroid and average time, the number of crystals, and a lateral moment describing the shower shape for the particle identification are calculated. The L3 trigger efficiency for Monte Carlo simulated events are shown in Tab. 2.6 for events that passed L1.

\begin{tabular}{lcccccc}
\hline \hline L3 trigger & $\epsilon_{b \bar{b}}$ & $\epsilon_{B \rightarrow \pi^{0} \pi^{0}}$ & $\epsilon_{B \rightarrow \tau \nu}$ & $\epsilon_{c \bar{c}}$ & $\epsilon_{u d s}$ & $\epsilon_{\tau \tau}$ \\
\hline 1 track filter & 89.9 & 69.9 & 85.5 & 89.2 & 88.2 & 94.1 \\
2 track filter & 98.9 & 84.1 & 94.5 & 96.1 & 93.2 & 87.6 \\
Combined DCH filters & 99.4 & 89.1 & 96.6 & 97.1 & 95.4 & 95.5 \\
2 cluster filter & 25.8 & 91.2 & 14.2 & 39.2 & 48.7 & 34.3 \\
4 cluster filter & 93.5 & 95.2 & 62.3 & 87.4 & 85.5 & 37.8 \\
Combined EMC filters & 93.5 & 95.7 & 62.3 & 87.4 & 85.6 & 46.3 \\
Combined DCH+EMC filters & $>99.9$ & 99.3 & 98.1 & 99.0 & 97.6 & 97.3 \\
Combined L1+L3 & $>99.9$ & 99.1 & 97.8 & 98.9 & 95.8 & 92.0 \\
\hline \hline
\end{tabular}

Table 2.6: L3 trigger efficiency (\%) for various physics processes, derived from Monte Carlo simulation.

\subsection{Monte Carlo Production and Data Flow}

A modern high-energy experiment can not prescind from a generator of simulated events that are as similar as possible as real data. The samples of simulated events, called Monte Carlo (MC) samples, can be used in physics analysis, for example, to test the procedures, estimate systematic errors, extract background shapes or expected number of events.

The production of the MC samples is divided in three main steps. The first one is the generation of the simulated $e^{+} e^{-}$final states in terms of a set of four-vectors, one for each 
particle. There are different types of generators, depending on the physical process that should be simulated, e.g. for $B-\bar{B}$ events the Jetset7 .4 [48] generator is used. The simulation of typical background events, as Bhabha scattering, bremsstralung, and also machine backgrounds and cosmic rays, is also included. The second step of MC production is the propagation of the generated particles through the detector, including the possible decays and all the processes of interactions with the material as energy loss and multiple scattering. The information relative to all the generated particles are referred to as "MC truth" and are available to the analysts. During the analysis this information can be used to study the selection, or a particular decay chain, matching the reconstructed tracks with the MC truth (truth matching). The third step consists of simulating the detector responses. The last two steps of the simulation are performed with the GEANT 4 [49] package, that allows a very detailed description of the detector. At this point the signals of the simulated events follow exactly the same path as real data, briefly described in the following.

When data events are collected (or simulated in case of MC) the first thing to do is the reconstruction of the event. This operation is performed in two steps and completed within twelve hours after data collection. The raw data obtained from the detector Data Acquisition System (DAQ) that passed Data Quality Manager (DQM) control, are stored in XTC (extended tagged container) files, one for each run. The first step of reconstruction is the Prompt Calibration that reconstructs only a subset of data and extracts the calibration constants that are stored in Condition Database (CDB), that also contains the detector systems conditions recorded during data taking. The second step is the full Event Reconstruction (ER) of all the XTC files, performed using the information in CDB. The output of the ER is written to data collections. During the years of operation of the detector, the detector calibrations and the algorithms for data reconstruction are constantly improved. At $B A B A R$ all the raw data were reprocessed with the improved algorithms about once a year.

The huge amount of collected data and simulated events makes it inefficient for all analysts to read the full sample. At BABAR there is a third production step, the skimming, where events passing different sets of physics-motivated criteria are written to separate streams, the skims. 


\section{Chapter 3}

\section{Lifetime Ratio Analysis Overview}

In this chapter we give an overview of the analysis: we present the reconstructed channels, the backgrounds, the fit strategy and the method to extract $y_{C P}$ and $\Delta Y$. We also provide a brief description of the previous published BABAR lifetime ratio analyses, and introduce the improvements ad optimizations to increase the precision of the measurement. Some of the details, not fundamental for the understanding of this overview, will become clear in the next chapters.

\subsection{General Outlook}

In order to measure $y_{C P}$ and $\Delta Y$ we need to determine the effective lifetimes of the $D^{0}$ and the $\bar{D}^{0}$ reconstructed in $C P$-even and $C P$-mixed final states. We reconstruct the $D^{0}$ and the $\bar{D}^{0}$ in the $C P$-even $K^{+} K^{-}$and $\pi^{+} \pi^{-}$, and in the $C P$-mixed $K^{ \pm} \pi^{\mp}$ final states.

The mixing parameter $y_{C P}$ is sensitive to the difference between the lifetime of the flavor eigenstates $\left(D^{0}\right.$ or $\left.\bar{D}^{0}\right)$ and the effective lifetime of the $D$ when it decays in a $C P$ eigenstates. In order to understand how this can be an evidence of mixing, let's suppose that $C P$ is conserved. In this case the free Hamiltonian eigenstates $D_{1}$ and $D_{2}$ are also $C P$ eigenstates. When, for instance, we reconstruct the $D$ in a $C P$-even final state, we select the $D_{1}$ component of the flavor eigenstate and therefore we measure the $D_{1}$ lifetime. When we reconstruct the $D$ in the $C P$-mixed final state we select a flavor eigenstate, and measure the $D^{0}$ (and $\bar{D}^{0}$ ) lifetime. In the case of mixing, the $D^{0}$ lifetime differs from the $D_{1}$ lifetime: the flavor eigenstate is a superposition of $D_{1}$ and $D_{2}$ and therefore its lifetime will be different from both the $D_{1}$ and $D_{2}$ lifetimes. In the case of no mixing, $D_{1}$ and $D_{2}$ are the same state, and therefore the $D^{0}$ lifetime will be the same as the $D_{1}$. In the more general case of $C P V, D_{1}$ and $D_{2}$ will differ from the $C P$ eigenstates but the $D^{0}$ lifetime will still be different from its effective lifetime when it is reconstructed in a $C P$ eigenstate. In principle we could use $C P$-odd final states as well (e.g. $\left.K_{S}^{0} \pi^{0}, K_{S}^{0} \omega, K_{S}^{0} \eta\right)$ and still be sensitive to mixing, but the $C P$-even modes are preferable from an experimental point 
of view since the reconstruction efficiency is higher. Another possible complication is the presence of $K_{S}^{0}$ in the final states: $C P V$ in the $K^{0}-\bar{K}^{0}$ system should then be disentangle from the $C P V$ in charm.

In this analysis we make no distinction between the Cabibbo-Favored (CF) $D^{0} \rightarrow K^{-} \pi^{+}$ and the doubly-Cabibbo-suppressed (DCS) $D^{0} \rightarrow K^{+} \pi^{-}$decays since they are both $C P$ mixed states. We also assume that we are not sensitive to $C P V$ in the $K^{ \pm} \pi^{\mp}$ decays. The SM predictions for $C P V$ in the CF $D^{0} \rightarrow K^{-} \pi^{+}$decay are greatly suppressed, since at first order it proceeds through a tree diagram. The ratio between the DCS and the CF decays is $\sim 410^{-3}$, and therefore the possible effects of $C P V$ in the DCS channel are also highly suppressed. We use the $K^{ \pm} \pi^{\mp}$ final states to measure the $D^{0}$ lifetime that is equal to the $\bar{D}^{0}$ lifetime in case of no $C P V$, therefore we do not distinguish $D^{0}$ from $\bar{D}^{0}$ decays to $K^{ \pm} \pi^{\mp}$. Hence we include the following decays in a single sample: $D^{0} \rightarrow K^{-} \pi^{+}$, $D^{0} \rightarrow K^{+} \pi^{-}, \bar{D}^{0} \rightarrow K^{+} \pi^{-}, \bar{D}^{0} \rightarrow K^{-} \pi^{+}$.

The measurement of $C P V$ consists in detecting different lifetimes of the $D^{0}$ decaying to $C P$-even eigenstates and the $\bar{D}^{0}$ decaying to the same final states. A different behavior of matter and antimatter in this context is an evidence of $C P$ violation. The reported evidence of $C P V$ by LHCb and CDF has been observed in the $D^{0} \rightarrow K^{+} K^{-}$and $D^{0} \rightarrow \pi^{+} \pi^{-}$ decays. They measure a difference of time-integrated asymmetries $\Delta A_{C P}$, mostly sensitive to direct $C P V$. Under our experimental assumptions the parameter $\Delta Y$, on the other hand, is sensitive to indirect $C P V$, therefore this measurement is mostly complementary to $\Delta A_{C P}$.

From data we extract the following three effective lifetimes:

- $\tau^{+}$for the $D^{0} \rightarrow h^{+} h^{-}$decays,

- $\bar{\tau}^{+}$for the $\bar{D}^{0} \rightarrow h^{+} h^{-}$decays,

- $\tau_{K \pi}$ for the $D^{0}$ and $\bar{D}^{0} \rightarrow K^{ \pm} \pi^{\mp}$ decays,

and use them to compute $y_{C P}$ and $\Delta Y$ :

$$
\begin{aligned}
y_{C P} & =\frac{\tau_{K \pi}}{2}\left[\frac{1}{\tau^{+}}+\frac{1}{\bar{\tau}^{+}}\right]-1, \\
\Delta Y & =\frac{\tau_{K \pi}}{2}\left[\frac{1}{\tau^{+}}-\frac{1}{\bar{\tau}^{+}}\right] .
\end{aligned}
$$

As indicated in Sec. 1.3, we assume that $K^{+} K^{-}$and $\pi^{+} \pi^{-}$candidates can share the effective lifetimes, $\tau_{K K}^{+}=\tau_{\pi \pi}^{+} \equiv \tau^{+}$and $\bar{\tau}_{K K}^{+}=\bar{\tau}_{\pi \pi}^{+} \equiv \bar{\tau}^{+}$. Another important experimental assumption is that the signal proper time distribution for all channels, deconvolved from the resolution function, is exponential. In other words, since the mixing rate is small $(\leq 1 \%)$, we neglect the effect of mixing on the decay rate (see Fig. $1.6 \mathrm{c}$ )). At BABAR, the oscillations due to mixing have been measured in the DCS $D^{0} \rightarrow K^{+} \pi^{-}$decays [17]. 
These events represent a very small fraction of the $K^{ \pm} \pi^{\mp}$ sample, and in addition the fraction of mixed decays is at most $1 \%$ of the DCS candidates.

\subsubsection{Reconstructed Signal Channels}

We analyze a data sample of $e^{+} e^{-}$collisions at the center of mass energy corresponding to the mass of the $\Upsilon(4 S)$, and select $e^{+} e^{-} \rightarrow c \bar{c}$ events. We use tagged candidates in which the $D^{0}$ comes from a $D^{*+} \rightarrow D^{0} \pi_{s}^{+}$decay, where the charge of the soft pion $\left(\pi_{s}^{+}\right)$indicates the flavor of the $D^{0}$ at production. In these decays the backgrounds are highly suppressed applying a cut on the variable $\Delta m$, defined as the difference between the reconstructed $D^{*+}$ and $D^{0}$ masses, $\Delta m=m_{D^{*}}-m_{D^{0}}$. The $m_{D^{0}}$ and $\Delta m$ distributions for the tagged $K^{ \pm} \pi^{\mp}$ channel are reported in Fig. 3.1. The resolution in $\Delta m$ is small, around $250 \mathrm{keV} / c^{2}$, and therefore is very effective in reducing the background.
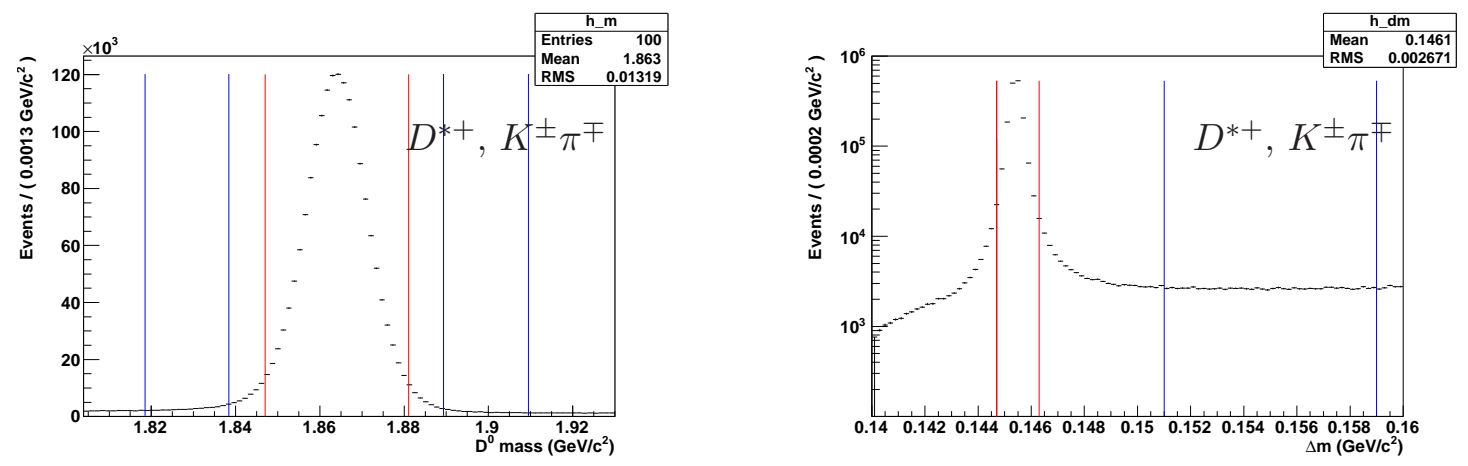

Figure 3.1: Distribution of mass (left) and $\Delta m$ (right) for the tagged $K^{ \pm} \pi^{\mp}$ channel. The red lines represent the signal region, the blue lines the sideband regions. Note the vertical logarithmic scale in the $\Delta m$ plot.

We use also untagged candidates in which the $D^{0}$ comes directly from the hadronization of the charm quark. These channels have $\sim 4$ times higher branching ratio than the corresponding tagged ones but do no benefit from the $\Delta m$ cut, therefore there is significantly more background. In Fig. 3.2 we report the reconstructed invariant mass distribution for the tagged and untagged $K^{+} K^{-}$final states: in the untagged channel there is clearly more background than in the tagged one. For this reason we do not use untagged $D^{0} \rightarrow \pi^{+} \pi^{-}$ decays. In summary, we reconstruct three tagged $\left(D^{*+} \rightarrow D^{0} \pi_{s}^{+}\right.$, with $D^{0} \rightarrow K^{ \pm} \pi^{\mp}$, $\left.K^{+} K^{-}, \pi^{+} \pi^{-}\right)$and two untagged $\left(D^{0} \rightarrow K^{+} K^{-}, K^{ \pm} \pi^{\mp}\right)$ decays.

In the untagged channels it is impossible to distinguish $D^{0}$ from $\bar{D}^{0}$ decays. As mentioned before, this is not a problem for the $K^{ \pm} \pi^{\mp}$ final state. For the untagged $K^{+} K^{-}$channel, on the other hand, we have to make an assumption on the number of $D^{0}$ and $\bar{D}^{0}$ decays present in the sample. We assume that half the candidates are $D^{0}$. Strictly speaking, for a mixing measurement, it is not crucial to distinguish $D^{0}$ from $\bar{D}^{0}$ since $y_{C P}$ is basically sensitive to the difference between the $D^{0}$ and the $D_{1}$ lifetimes. However in this analysis we are also measuring a $C P V$ parameter, $\triangle Y$, and therefore we need to distinguish the 

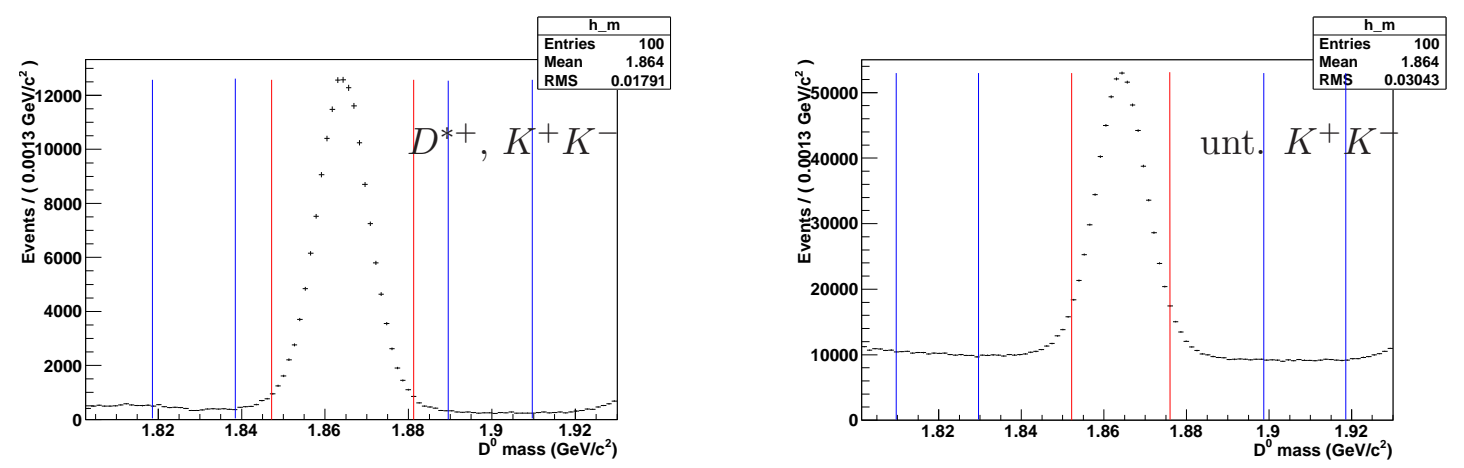

Figure 3.2: Distribution of reconstructed mass for the tagged (left) and untagged (right) $K^{+} K^{-}$channels. The red lines represent the signal region, the blue lines the low and high sidebands.

$D^{0}$ decays to $C P$-even eigenstates from the $\bar{D}^{0}$ decays to the same final states.

The determination of the $D^{0}$ final state depends on the identification of the tracks. At $B A B A R$ the particle identification is performed using the $\mathrm{d} E / \mathrm{d} x$ measurements in the SVT and the DCH for tracks below $700 \mathrm{MeV} / c$. The high momentum tracks are identified with a dedicated detector, the DIRC, that measures the Cherenkov angle associated to the track. The SVT and DCH also provide the measurement of the track trajectory that allow one to associate the track with its Cherenkov angle.

\subsubsection{Proper Time Measurement}

The three effective lifetimes are simultaneously extracted from an extended unbinned maximum likelihood (ML) fit to the measured proper time $t$ and its uncertainty $\sigma_{t}$ of the $D^{0}$ candidates reconstructed in the five channels.

Once the $D^{0}$ candidate decay tree is reconstructed, the proper time and its error are determined using the measured decay length, $\ell$, and boost, $\beta \gamma$, since:

$$
\ell=\beta \gamma t .
$$

In particular, the decay length is determined with the tracking system that allows one to reconstruct the $D^{0}$ production and decay vertices, while the $\beta \gamma$ is computed from the resulting momentum of the $D^{0}$ daughter tracks, measured in the $1.5 \mathrm{~T}$ magnetic field mainly with the DCH.

\subsubsection{Mass and Proper Time Correlation of the Signal Candidates}

There is a correlation between the reconstructed proper time and the reconstructed mass for the $D^{0}$ signal candidates. This is illustrated in Fig. 3.3 which shows the average of the proper time as a function of the mass for the tagged $K^{ \pm} \pi^{\mp}$ events. 


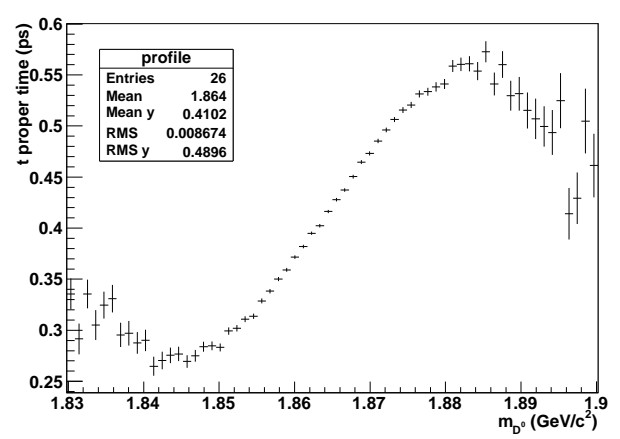

Figure 3.3: Average of the reconstructed proper time in bins of reconstructed mass for the tagged $K^{ \pm} \pi^{\mp}$ channel.

The almost linear correlation between $m_{D^{0}}=1.85 \mathrm{GeV} / c^{2}$ and $m_{D^{0}}=1.88 \mathrm{GeV} / c^{2}$ can be easily understood. Decays reconstructed with too high proper time will have a vertex further away from the interaction point than the true vertex, this results in a too large reconstructed opening angle, $\theta$, and thus mass, since:

$$
m_{D^{0}}^{2}=m_{1}^{2}+m_{2}^{2}+2\left(E_{1} E_{2}-p_{1} p_{2} \cos \theta\right)
$$

where $m_{i}, E_{i}$ and $p_{i}$ are the mass, energy and momentum of the the $D^{0}$ daughter tracks. Similar events with too low proper time have smaller opening angles and therefore too low mass. The correlation breaks down further away from the true $D^{0}$ mass because those decays typically are poorly reconstructed for other reasons.

In the range $1.85<m_{D^{0}}<1.88 \mathrm{GeV} / c^{2}$ the correlation is almost linear. MC studies show that, for a given final state, the correlation in the tagged channel is compatible with the correlation in the untagged channel. We study these correlation in data, in the lowbackground tagged channels, and find that they are well described by an odd function, a third order polynomial centered in the middle of the $m_{D^{0}}$ range. We fit data and find that the coefficient of the first order polynomial is $(8.6 \pm 0.6) \mathrm{fs} /\left(\mathrm{MeV} / \mathrm{c}^{2}\right)$ for $\pi^{+} \pi^{-}$, $(10.8 \pm 0.1) \mathrm{fs} /\left(\mathrm{MeV} / \mathrm{c}^{2}\right)$ for $K^{ \pm} \pi^{\mp}$ and $(14.4 \pm 0.4) \mathrm{fs} /\left(\mathrm{MeV} / \mathrm{c}^{2}\right)$ for $K^{+} K^{-}$. In Fig. 3.4 we report the average of the reconstructed proper time in bins of reconstructed $D^{0}$ mass for the three tagged and the two untagged channels evaluated on signal truth-matched MC events, in different colors for the different final states.

To suppress the background we apply a cut on the reconstructed mass, and define a signal region. This correlation is potentially dangerous because the cut on the reconstructed mass affects also the proper time. Since the correlations are different for different final states, this can have an impact also on our observables, in particular on $y_{C P}$. In order to minimize the effects of this correlation on $y_{C P}$ and $\Delta Y$ we perform a data-driven optimization of the signal region in mass, described in Sec. 4.3. 

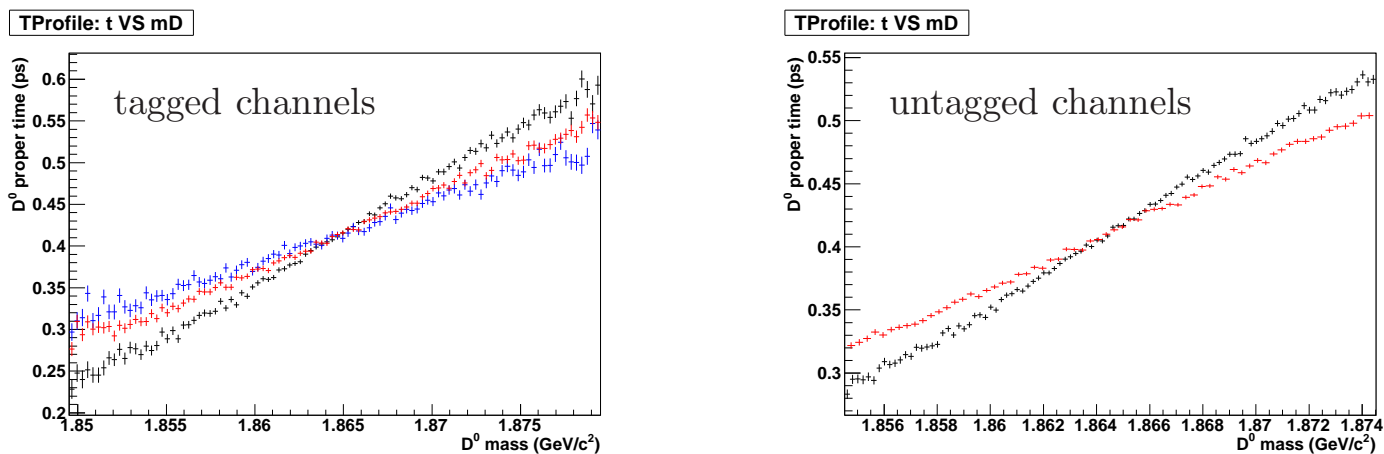

Figure 3.4: Average of the reconstructed proper time in bins of reconstructed $D^{0}$ mass for truth-matched signal MC events. In the left plot we report the tagged channels and in the right plot the untagged channels: $K^{ \pm} \pi^{\mp}$ in red, $K^{+} K^{-}$in black and $\pi^{+} \pi^{-}$in blue.

\subsubsection{Backgrounds}

The background candidates that pass the selection are classified into two major categories:

- misreconstructed-charm background:

this category includes $D^{0}$ candidates whose daughter tracks share as a common ancestor a long-living charm meson. This background is very small $(\leq 1 \%)$ compared to the combinatorial background, but pernicious since it is a signal-like component.

- combinatorial background:

this category includes all the background candidates that do not belong to the misreconstructed-charm category, mainly random combination of tracks.

The wrongly tagged signal candidates, the ones in which the soft pion has been misreconstructed, are considered as signal events, and the signal probability density function (PDF) takes them into account.

The misreconstructed-charm events are not distinguishable from signal events in a fit to the proper time or the mass, and therefore we describe them using the simulated events. We extract the shape and the expected number of events from MC datasets. A systematic error is associated to this assumption. On the other hand, the shape and the expected number of events for the combinatorial background are estimated from data. A more detailed description of the compositions of these backgrounds and the functional forms used to fit their reconstructed proper time distributions is discussed in Chapter 5 .

\subsubsection{Simultaneous Fit}

Since the proper time resolution is in principle different for the five channels, and since we need to distinguish between the $D^{0}$ and $\bar{D}^{0}$ candidates for the tagged $C P$-even modes, the five channels are divided into seven modes: the two untagged channels, the tagged $K^{ \pm} \pi^{\mp}$ channel, the tagged $D^{0} \rightarrow K^{+} K^{-}, \bar{D}^{0} \rightarrow K^{+} K^{-}, D^{0} \rightarrow \pi^{+} \pi^{-}, \bar{D}^{0} \rightarrow \pi^{+} \pi^{-}$channels. 
We then perform an extended unbinned ML simultaneous fit to the $\left(t, \sigma_{t}\right)$ distributions of the seven modes in order to extract the three lifetimes needed to compute $y_{C P}$ and $\Delta Y$.

\section{Fit Regions}

As mentioned before, we fit the $D^{0}$ candidates in a selected region of the reconstructed mass $m_{D^{0}}$, or $\left(m_{D^{0}}, \Delta m\right)$ for the tagged channels. These regions are shown in Fig. 3.1 and Fig. 3.2 with vertical red lines. The signal region has been optimized in order to minimize the effects of the proper time - mass correlation, but also the statistical error on the lifetimes, as explained in Sec. 4.3.

In the analysis we also make use of sidebands in order to obtain the shape for the combinatorial events. The sidebands are shown in Fig. 3.1 and Fig. 3.2 with vertical blue lines. In Fig. 3.5 we report the two-dimensional $\left(m_{D^{0}}, \Delta m\right)$ distribution with the indication of the signal and sideband region for the tagged $K^{ \pm} \pi^{\mp}$ data.

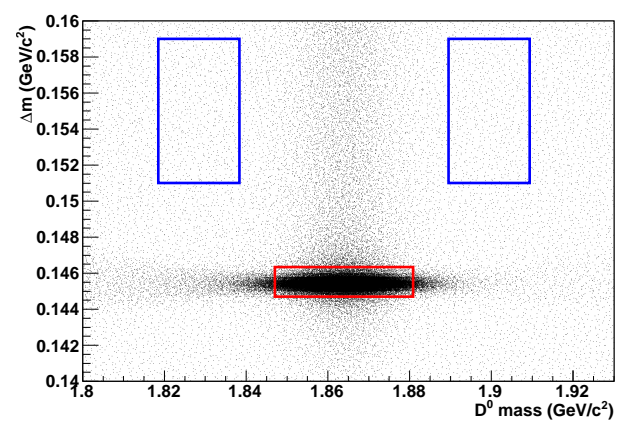

Figure 3.5: Two-dimensional plot of the $\left(m_{D^{0}}, \Delta m\right)$ distribution of the tagged $K^{ \pm} \pi^{\mp}$ events. The red box represents the signal region, the blue ones the sidebands.

\section{Mass Fits}

A preliminary step before the final fit to $\left(t, \sigma_{t}\right)$ is fitting the mass distributions. These fits allow one to extract the expected number of background events, this is information that is needed in the final fit. The untagged $K^{+} K^{-}$channel represents an exception: the mass fit prediction was not accurate enough and we decided to extract the number of combinatorial events directly from the final fit to $\left(t, \sigma_{t}\right)$, instead of fixing it as in all the other channels.

\section{Final Fit}

In the final fit the shape and the expected number of events for the two background categories of each channel are fixed, except the expected combinatorial yields in the untagged $K^{+} K^{-}$channel. The signal PDF is a simultaneous PDF with seven components, one 
for each mode. Most of the resolution function parameters are shared among the seven modes. Differences in reconstruction among the modes are described by few additional parameters.

The three lifetimes, along with the signal resolution function parameters and the expected number of signal events in each mode and for the untagged $K^{+} K^{-}$combinatorial events are extracted from an extended unbinned ML fit. The fit is performed in a signal region in $D^{0}$ mass for the untagged and $\left(m_{D^{0}}, \Delta m\right)$ for the tagged samples.

The values of the extracted lifetimes are used to compute $y_{C P}$ and $\Delta Y$, and their statistical errors are computed using the covariance matrix returned from the fit.

To avoid potential bias, we finalize the selection criteria, the fitting procedures and the determination of the systematic uncertainties prior to examining the extracted lifetimes. During the analysis we therefore blind the values of the three lifetimes by the addition of an unknown quantity, different for each lifetime parameter and associated with a blinding string. This way we blind both $y_{C P}$ and $\Delta Y$.

\subsection{Previous BABAR Analyses}

The BABAR experiment has carried out an important and comprehensive physics program, with the main goal of studying $C P$ violation in the $B$ meson system. A considerable part of the program was also devoted to charm physics, and the search for $D^{0}-\bar{D}^{0}$ mixing and $C P V$ in the charm sector had become an important item for the experiment since the accumulated statistics became relevant. In the past years $B A B A R$ published three analyses reporting evidence of $D^{0}$ mixing and a search for $C P V$ in the $D^{0}$ decays.

The first evidence of mixing [17] in the DCS channel $D^{0} \rightarrow K^{+} \pi^{-}$was published in 2007 . In this analysis the modifications to the decay rate due to mixing were analyzed and measured. One year later the first sensitive $y_{C P}$ analysis [50] was published, confirming the evidence of mixing and reporting no evidence of $C P V$. This analysis was performed using tagged channels only and the total error was dominated by the statistical one. In 2009 a new $y_{C P}$ analysis was published [51], this time only untagged channels were used, and no $C P V$ measurement was possible. The value from the untagged measurement was combined with the one from the tagged analysis yielding the most significant mixing measurement at that time.

The previous tagged and untagged analyses are very similar in terms of the event selection, the general procedure and the fit. In the following we give a brief review of the two measurements, performed on $384 \mathrm{fb}^{-1}$ which corresponds to around $480 \mathrm{M}$ of $e^{+} e^{-} \rightarrow c \bar{c}$ events. 


\section{Event Selection}

A standard selection was applied, besides selection criteria improving the quality of the tracks and removing $D^{0}$ decays from $B$ mesons, a cut on the helicity angle of the $D^{0}$ was applied. The helicity angle is the angle between the $D^{0}$ direction in the laboratory frame and the positive daughter in the $D^{0}$ rest frame. Its distributions peaks at -1 and +1 for the combinatorial events. This cut was effective in reducing the combinatorial background but it also removed a non-negligible fraction of signal events.

Another important point of the selection was the choice of the mass window. As explained in Sec. 3.1.3 there is a correlation between the reconstructed invariant mass and the reconstructed proper time. The choice of the mass window can therefore have an impact on the extracted lifetimes. Since the values of the correlations depend on the a final state, this can also have an impact on the measured $y_{C P}$. In the previous analyses the mass window was chosen on the simulated events and no dedicated studies were performed to minimize the effects of this correlation on data.

\section{Characterization of the $D^{0}$ Candidates and Fit}

The lifetime values were extracted with an extended unbinned ML fit to the proper time and proper time error two-dimensional distribution. The misreconstructed-charm background PDF was extracted from simulated events, while the combinatorial PDF was extracted from data sidebands. In the tagged analysis only one sideband was used, while in the untagged one, a low and high mass sidebands were used. The combinatorial PDF did not contain a proper time error dependent part, hence the correlations between proper time and proper time error were neglected. In the final fit the backgrounds PDF shapes and expected yields were fixed, while the signal resolution functions and lifetimes were all floating.

\section{Results and Main Systematic Errors}

In Tab. 3.1 we report the results of both analyses and the combined value of $y_{C P}$, that excluded the no-mixing hypothesis with a significance of 4.1 standard deviation. No evidence of $C P$ violation was found while a value of $y_{C P}$ at the level of $1 \%$ was measured in both analysis.

The precision of both measurements was limited by the statistics of the data sample. The untagged analysis could exploit a larger number of $D^{0}$ candidates, hence the statistical error is smaller, but the presence of more backgrounds increased the systematic error.

In Tab. 3.2 we report the summary of the systematic errors for the tagged and untagged analysis. An important source of systematic error in both analysis was related to the choice of the mass window, the main contribution in the "Signal" row. Another important 


\begin{tabular}{l|cc}
\hline \hline Sample & $y_{C P}(\%)$ & $\Delta Y(\%)$ \\
\hline Tagged & $1.24 \pm 0.39 \pm 0.13$ & $-0.26 \pm 0.36 \pm 0.08$ \\
Untagged & $1.12 \pm 0.26 \pm 0.22$ & - \\
\hline Combined & $1.16 \pm 0.22 \pm 0.18$ & - \\
\hline \hline
\end{tabular}

Table 3.1: Summary of the previous 2007 and 2009 BABAR $y_{C P}$ and $\Delta Y$ results. The first error is statistical, the second systematic. The average is obtained by combining the tagged result with the untagged result using the BLUE method [52], assuming all the systematic errors from the tagged analysis are 100\% correlated with those from the untagged analysis.

\begin{tabular}{c|cc|c}
\hline \hline \multirow{2}{*}{ Systematic } & \multicolumn{2}{|c|}{$\begin{array}{c}\text { tagged } \\
\text { analysis }\end{array}$} & $\begin{array}{c}\text { untagged } \\
\text { analysis }\end{array}$ \\
& $\Delta\left[y_{C P}\right](\%)$ & $\Delta(\Delta Y)$ & $\Delta\left[y_{C P}\right](\%)$ \\
\hline Signal & 0.085 & 0.062 & 0.111 \\
Charm & 0.043 & 0.001 & 0.086 \\
Combinatorial & 0.045 & 0.002 & 0.115 \\
Selection & 0.046 & 0.011 & 0.071 \\
Detector & 0.064 & 0.054 & 0.093 \\
\hline Total & 0.132 & 0.083 & 0.216 \\
\hline \hline
\end{tabular}

Table 3.2: Summary of systematic errors on $y_{C P}$ and $\Delta Y$ in the tagged (left) and untagged (right) analyses. The individual systematic errors were added in quadrature to evaluate the total systematic error.

contribution in the untagged analysis was the one related to the combinatorial background. Although the misreconstructed-charm background was a very small fraction of the selected candidates, the systematic error associated with it was not negligible. No systematic error associated with the helicity cut was included.

\subsection{Analysis Improvements and Optimizations}

The analysis described in this thesis represents the final measurements of $y_{C P}$ and $\Delta Y$ with the BABAR dataset. The precision of the previous measurements were limited by the finite statistics of the data sample. We have performed an optimization of the selection aimed at increasing signal efficiency, but keeping the background levels under control, especially for the untagged channels.

We have loosened the selection criteria, described in detail in Sec. 4.2. In particular we have removed the cut on the helicity angle and chosen a looser track selector. The analysis also benefits from an integrated luminosity increase of $\sim 20 \%$ with respect to the data sample exploited in the previous analyses, and an improved official BABAR reconstruction code. These changes result in an increase of signal candidates of almost $90 \%$, projecting 
the statistical error down to around $0.18 \%$ for $y_{C P}$ and $0.29 \%$ for $\Delta Y$.

We have also performed a data-driven optimization of the signal region, described in Sec. 4.3, that allowed a reduction of the associated systematic error.

We have implemented a more detailed parameterization of the backgrounds. For what concerns the misreconstructed-charm background, we could exploit a much larger sample of simulated events, corresponding to ten times the data sample. We have included the proper time error in the combinatorial description and used two mass sidebands both for the tagged and the untagged channels.

Finally, we have performed a simultaneous fit to the seven modes instead of an a posteriori combination of the results coming from two independent fits. The statistical precision of the measurement actually benefits from the simultaneous fit only if we use the same resolution function parameters to describe all the seven modes, in that case the number of parameters extracted from the fit is reduced and the statistical error is reduced. Tagged candidates are therefore reconstructed using the same information of the untagged candidates, i.e. not considering the soft pion information; this allows one to share the signal resolution function among the five channels. 



\section{Chapter 4}

\section{Event Selection}

In this chapter we present the data and simulated events samples used to perform and validate the analysis. We describe the reconstruction and the selection of the signal candidates, in particular the optimization of the reconstructed-mass window. Finally we present the expected number of signal and background events in the signal region obtained from fits to the invariant-mass distributions.

\subsection{Data and Monte Carlo Samples}

The data sample used in this analysis corresponds to an integrated luminosity of $468.2 \mathrm{fb}^{-1}$ collected by the BABAR experiment at, and slightly below, the $\Upsilon(4 S)$ resonance. The onand off-resonance luminosities for each running period (Run1-6) are shown in Tab. 4.1. We have not used data sample collected at either the $\Upsilon(2 S)$ or $\Upsilon(3 S)$ because preliminary studies on those samples have shown different background levels and signal efficiencies with respect to the $\Upsilon(4 S)$ sample, and the gain in total luminosity was judged not worth the systematic difference among the samples.

\begin{tabular}{cccc}
\hline \hline Period & Off-peak $\mathcal{L}\left[\mathrm{fb}^{-1}\right]$ & On-peak $\mathcal{L}\left[\mathrm{fb}^{-1}\right]$ & Total $\mathcal{L}\left[\mathrm{fb}^{-1}\right]$ \\
\hline Run 1 & 2.56 & 20.37 & 22.93 \\
Run 2 & 6.87 & 61.32 & 68.19 \\
Run 3 & 2.44 & 32.28 & 34.72 \\
Run 4 & 10.02 & 99.61 & 109.63 \\
Run 5 & 14.28 & 132.36 & 146.64 \\
Run 6 & 7.75 & 78.31 & 86.06 \\
\hline Total & 43.92 & 424.25 & 468.17 \\
\hline \hline
\end{tabular}

Table 4.1: Luminosities of Run1 to Run6 data samples.

In order to extract the values of $y_{C P}$ and $\Delta Y$ from data we need information from the $B A B A R$ simulated events. The MC samples are used to determine the shape of some 
background probability density functions (PDF), as well as to validate the fit and the analysis procedure. The sizes of the generic Monte Carlo samples used in this analysis are listed in Tab. 4.2.

\begin{tabular}{lrr}
\hline \hline Channel & Generated & $\mathcal{L}\left[\mathrm{fb}^{-1}\right]$ \\
\hline$e^{+} e^{-} \rightarrow c \bar{c}$ & 6060721000 & 4654.4 \\
$e^{+} e^{-} \rightarrow u \bar{u}, d \bar{d}, s \bar{s}$ & 3958668000 & 1894.1 \\
$e^{+} e^{-} \rightarrow B^{+} B^{-}$ & 2331948000 & 4243.8 \\
$e^{+} e^{-} \rightarrow B^{0} \bar{B}^{0}$ & 2366003000 & 4305.7 \\
$e^{+} e^{-} \rightarrow \tau^{+} \tau^{-}$ & 831508000 & 884.6 \\
\hline \hline
\end{tabular}

Table 4.2: Summary of the generic Monte Carlo samples used in this analysis. The luminosities are calculated using the cross sections given in Tab. 2.1. The equivalent luminosities for the $e^{+} e^{-} \rightarrow B^{+} B^{-}$and $B^{0} \bar{B}^{0}$ samples are computed assuming that the branching ratios for the two processes are equal.

As a preliminary operation we randomly split each sample corresponding to a particular process $\left(e^{+} e^{-} \rightarrow c \bar{c}, B^{+} B^{-}, \ldots\right)$ in $476 \mathrm{fb}^{-1}$ units $^{1}$. The units are then merged together to form the datasets used through all the analysis for the various kinds of fits, as described in the following.

\section{Datasets for the Data Fits}

In the final fit to data we use some information from simulated events to determine the background PDFs. We build one MC dataset for each of the three categories (signal, misreconstructed charm and combinatorial), containing all the relevant units at our disposal. Signal and misreconstructed-charm candidates only come from $e^{+} e^{-} \rightarrow c \bar{c}$ simulated events while combinatorial candidates have contribution from all types of simulated events, we therefore built the following MC datasets:

- bigSignal $\left(4.65 \mathrm{ab}^{-1}\right)$ : contains truth-matched (see Sec. 2.9) signal candidates (from all $e^{+} e^{-} \rightarrow c \bar{c}$ simulated events);

- bigCharm $\left(4.65 \mathrm{ab}^{-1}\right)$ : contains truth-matched misreconstructed-charm candidates (from all $e^{+} e^{-} \rightarrow c \bar{c}$ simulated events);

- bigCombin $\left(1.89 \mathrm{ab}^{-1}\right)$ : contains truth-matched combinatorial candidates (limited by the smallest class of simulated events $\left.{ }^{2}, e^{+} e^{-} \rightarrow u \bar{u}, d \bar{d}, s \bar{s}\right)$;

\footnotetext{
${ }^{1}$ the luminosity used is slightly higher than data luminosity $(+1.7 \%)$ because this operation was done at an early stage of the analysis, when the luminosity of the data sample was not correctly computed.

${ }^{2}$ the $e^{+} e^{-} \rightarrow \tau^{+} \tau^{-}$contribution after selection is negligible.
} 


\section{Datasets for the MC Fits}

The available amount of MC allows us to build 4 independent mixed datasets (cocktails) to test the fit, each equivalent to the data luminosity. In order to repeat the exact procedure that will be performed on data, we also need three MC datasets (one for each category) that are independent of the corresponding cocktail. For each MC cocktail used for the fit, cocktailX $\left(476 \mathrm{fb}^{-1}\right)$, we build the correspondent and independent bigSignalX $\left(4.18 \mathrm{ab}^{-1}\right)$, bigCharmX $\left(4.18 \mathrm{ab}^{-1}\right)$ and bigCombinX $\left(1.42 \mathrm{ab}^{-1}\right)$, with $\mathrm{X}=1,2,3,4$.

\section{Datasets for the Signal-Only Fits}

In order to test the signal description and the feasibility of the simultaneous fit, we also have signal-only MC datasets. Since we have a large amount of $e^{+} e^{-} \rightarrow c \bar{c}$ simulated events we have the possibility to build 9 independent datasets, oneSignalX (with $\mathrm{X}=1, \ldots, 9$ ), $476 \mathrm{fb}^{-1}$ each.

In Fig. 4.1 we report the comparison of data and $\mathrm{MC}$ mass distributions for the tagged $K^{ \pm} \pi^{\mp}$ and the untagged $K^{+} K^{-}$channels, the other plots can be found in Appendix B. There is a good agreement between data and the simulated events.
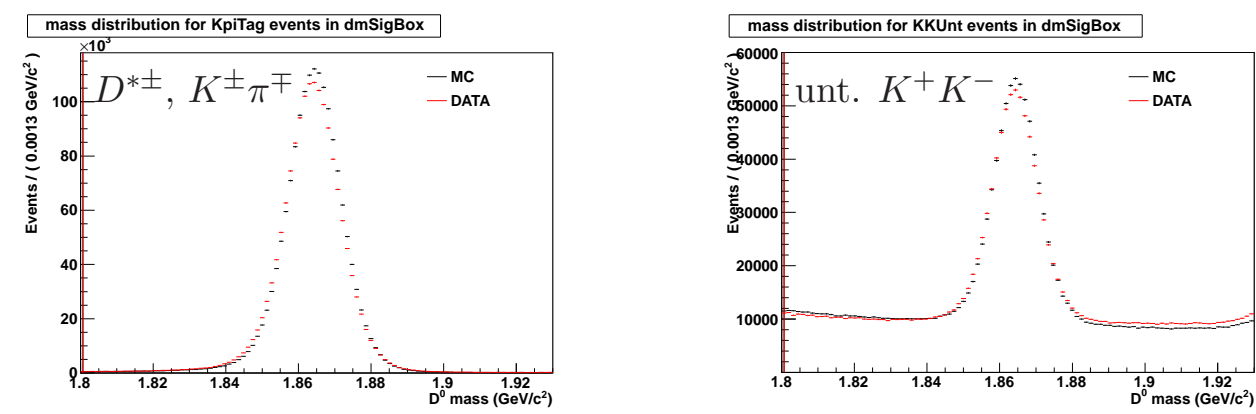

Figure 4.1: Mass distribution for data (red) and MC (black) for the tagged $K^{ \pm} \pi^{\mp}$ (left) and the untagged $K^{+} K^{-}$channels (right). The MC distribution is scaled to data luminosity.

\subsection{Reconstruction and Selection Criteria}

To perform an optimal measurement of $y_{C P}$ and $\Delta Y$, we reconstruct the $D^{0}$ two-body decays in the following seven modes, five tagged (4.1 to 4.5) and two untagged (4.6 and 
4.7):

$$
\begin{array}{rll}
D^{*+} \rightarrow \pi_{s}^{+} D^{0} \quad, \quad D^{0} \rightarrow K^{+} K^{-} \\
D^{*-} \rightarrow \pi_{s}^{-} \bar{D}^{0} \quad, \quad \bar{D}^{0} \rightarrow K^{+} K^{-} \\
D^{*+} \rightarrow \pi_{s}^{+} D^{0} \quad, \quad D^{0} \rightarrow \pi^{+} \pi^{-} \\
D^{*-} \rightarrow \pi_{s}^{-} \bar{D}^{0} \quad, \quad \bar{D}^{0} \rightarrow \pi^{+} \pi^{-} \\
D^{*+} \rightarrow \pi_{s}^{+} D^{0} \text { and } D^{*-} \rightarrow \pi_{s}^{-} \bar{D}^{0} \quad, \quad D^{0} \text { and } \bar{D}^{0} \rightarrow K^{-} \pi^{+} \text {and } K^{+} \pi^{-} \\
\text {no tag } \quad, \quad D^{0} \text { and } \bar{D}^{0} \rightarrow K^{+} K^{-} \\
\text {notag } \quad, \quad D^{0} \text { and } \bar{D}^{0} \rightarrow K^{-} \pi^{+} \text {and } K^{+} \pi^{-}
\end{array}
$$

The decays (4.1) to (4.4) and (4.6), are to $C P$-even final states. We use these $D^{0}$ candidates to obtain the effective lifetimes $\tau^{+}$and $\bar{\tau}^{+}$.

The decays (4.5) and (4.7) are to $C P$-mixed final states and involve $\mathrm{CF}\left(D^{0} \rightarrow K^{-} \pi^{+}\right)$ and DCS $\left(D^{0} \rightarrow K^{+} \pi^{-}\right)$decays. For the purpose of the analysis, we do not need to distinguish between the two. We collect both the DCS and the CF candidates in same dataset and describe them with a single PDF. We use these modes to obtain the $D^{0}$ lifetime $\tau_{K \pi}$.

There may be more than one candidate decay in a given event. All candidates not sharing daughter tracks between them are retained. If an event contains a tagged decay, all candidates from that event are eliminated from the untagged sample. The adjudication procedure is described in detail in Section 4.2.2.

Since the majority of the events in the skim do not contain a tagging $D^{*}$, we reskim the data, writing the tagged events into one collection and the untagged events into another. If an event has both a tagged and an untagged candidate, it is written to both collections. Removal of tagged candidates from the untagged sample is done after the final selection. The skimming and reskimming selection criteria are described in Section 4.2.1.

We next read the reskimmed collections containing a $D^{*}$ tag or a untagged $D^{0}$ and write a "primary" ntuple containing information about the decays of each. One of the important operations performed at this stage is truth matching the Monte Carlo simulated events. No additional selection criteria are imposed at this stage.

In the final stage of event selection we apply a final set of selection criteria, including the adjudication of multiple candidates with shared daughter tracks. These criteria are useful to suppress the background and improve the quality of the signal candidates, and are described in Section 4.2.2.

\subsubsection{Skim and Reskim Selections}

The skim is a collection of reconstructed events obtained applying physics-motivated loose selection criteria to the sample of all the reconstructed events. The selection criteria of 
the skim used for this analysis are summarized in the following.

We select oppositely charged pion and kaon candidates satisfying very loose requirements on particle identification (PID) and fit them to a common vertex with a $\chi^{2}$ probability from the $D^{0}$ vertex fit, $P\left(\chi_{D^{0}}^{2}\right)$, satisfying $0.005<P\left(\chi_{D^{0}}^{2}\right)<1.0$. In order to remove the $D^{0}$ coming from $B$ decays, we retain $D^{0}$ candidates with a center-of-mass momentum $2.4 \mathrm{GeV} / c \leq p_{D^{0}}^{*}<10 \mathrm{GeV} / c$. In case of the tagged channels, we combine the $D^{0}$ candidate with a slow pion candidate, imposing a constraint on the position of the vertex, that should lie within the $e^{+} e^{-}$interaction region (beam constraint). This requirement improves the measurement of the slow pion track parameters. We retain the resulting $D^{*}$ candidates that satisfy $0.13 \mathrm{GeV} / c^{2}<\Delta m<0.16 \mathrm{GeV} / c^{2}$.

Next we reskim the events to further reduce the backgrounds and improve the efficiency of the $D^{*}$-tagged signal candidates. We require the invariant mass of the $D^{0}$ daughter tracks combination calculated prior to the vertex fit, $m_{K \pi}^{\text {pre }}$, to satisfy $1.7745 \mathrm{GeV} / c^{2}<$ $m_{K \pi}^{\text {pre }}<1.9545 \mathrm{GeV} / c^{2}$. We fit each of the candidates in the pair to a common vertex, with $0.0005<P\left(\chi_{D^{0}}^{2}\right)<1.0$. We require the reconstructed $D^{0}$ mass satisfy $1.7745<$ $m_{D^{0}}<1.9545 \mathrm{GeV} / c^{2}$, and the $D^{0}$ center-of-mass momentum $p_{D^{0}}^{*} \geq 2.4 \mathrm{GeV} / c$. In case of the tagged channels, we select a slow pion candidate which does not overlap with the $D^{0}$ daughter tracks and with a center-of-mass momentum $p_{\pi_{s}}^{*}<0.45 \mathrm{GeV} / c$. We invalidate the previous $D^{0}$ vertex fit and refit each $D^{0} \quad$ (and $D^{0}$ with a slow pion in the case of $D^{*}$ candidates) to a common vertex, imposing a beam constraint. This requirement also improves the measurement of the slow pion track parameters. Finally we require that $P\left(\chi^{2}\right)$ from the vertex fit satisfy $0.0005<P\left(\chi^{2}\right)<1.0$ and retain all $D^{0}$ candidates and the resulting $D^{*}$ candidates with $0.13 \mathrm{GeV} / c^{2}<\Delta m<0.16 \mathrm{GeV} / c^{2}$.

\subsubsection{Final Selection}

When making the final ntuple we apply further selection criteria on the $D^{0}$ candidates. The reconstructed $D^{0}$ invariant mass is required to satisfy $1.80 \mathrm{GeV} / c^{2} \leq m_{D^{0}}<1.93 \mathrm{GeV} / c^{2}$, and the $D^{0}$ center-of-mass momentum $p_{D^{0}}^{*} \geq 2.5 \mathrm{GeV} / c$. We retain $D^{0}$ candidates with $\chi^{2} D^{0}$-vertex probability satisfy $0.001 \leq P\left(\chi^{2}\right) \leq 1$. In order to improve the quality of the $D^{0}$ daughter tracks, we require the number of their $\mathrm{DCH}$ hits $N_{K, \pi}^{\mathrm{DCH}} \geq 12$, and that they satisfy loose PID selector requirements. Additional requirements are imposed for $D^{*}$ candidates. We reject poorly measured slow pion tracks requiring the momentum in the laboratory reference frame $p_{\pi_{\mathrm{s}}}>0.1 \mathrm{GeV} / c$, and the number of SVT and DCH hits of the slow pion track, $N_{\pi_{\mathrm{s}}}^{\mathrm{SVT}}$ and $N_{\pi_{\mathrm{s}}}^{\mathrm{DCH}}$, satisfy $N_{\pi_{\mathrm{s}}}^{\mathrm{SVT}} \geq 6$ and $N_{\pi_{\mathrm{s}}}^{\mathrm{DCH}}>0$, respectively. We also require slow pion to be inconsistent with electron. Only candidates satisfying $0.14 \mathrm{GeV} / c^{2} \leq \Delta m<0.16 \mathrm{GeV} / c^{2}$ are retained. 


\section{Creating Independent Tagged and Untagged Datasets}

We construct an untagged sample which is totally disjoint from the tagged sample. This is done by recording the timestamps of all events containing one or more tagged $D^{0}$ candidate decays passing the selection criteria. An event's timestamp is recorded only if $0.1447<\Delta m<0.1463 \mathrm{GeV} / c^{2}$ (corresponding to the signal region cut, Eq. (4.8)). If the timestamp of the event containing the current untagged $D^{0}$ candidate appears in the list of tagged candidate event timestamps, then that $D^{0}$ is rejected. We don't simply remove the $D^{0}$ coming from one of the tagged decays, we remove all the $D^{0}$ candidates in an event containing a tagged candidate.

\section{Overlapping $D^{*}$ and $D^{0}$ Candidates}

Two $D^{0}$ candidates (or $D^{*}$ candidates in the case of the tagged channels) are overlapping if one or more of the daughter tracks of the first candidate are also daughters of the second candidate. The number of events with one or more $D^{0}\left(D^{*}\right)$ candidate pairs is around $0.5 \%$ in all channels except in the untagged $K^{+} K^{-}$case, for which it is $0.04 \%$. The adjudication criterion is to retain the candidate with the highest overall $\chi^{2}$ probability of the vertex fit and reject all the other overlapping candidates.

\subsection{Signal and Sidebands Regions Definition}

The main systematic error in the previous analyses was related to the choice of the mass window, as mentioned in Sec. 3.2. The two effects mainly responsible for it are:

- the proper time - mass correlation (explained in Sec. 3.1.3);

- the different amount of background events when selecting different signal region widths.

The choice of the signal region width has also an impact on the statistical error.

In this analysis we perform a dedicated study in order to minimize the total error on the lifetimes, in particular the effect of the correlation between the mass and the proper time. The procedure and main results of the study are reported in the next sections.

For what concerns the tagged events, the cut on $\Delta m$ for the signal region and the sidebands is taken from the previous tagged analysis and not investigated since it has no direct impact in the systematic error:

$$
\begin{array}{rr}
\text { signal region: } & 0.1447 \mathrm{GeV} / c^{2}<\Delta m<0.1463 \mathrm{GeV} / c^{2} \\
\text { sideband region: } & 0.151 \mathrm{GeV} / c^{2}<\Delta m<0.159 \mathrm{GeV} / c^{2} \\
\text { full region: } & 0.14 \mathrm{GeV} / c^{2}<\Delta m<0.16 \mathrm{GeV} / c^{2} .
\end{array}
$$


Also the sidebands definition had a negligible impact on the systematic error in both the previous analyses. We therefore keep the same definition as in the previous analyses, $20 \mathrm{MeV} / c^{2}$ wide regions, $44.5(35.5) \mathrm{MeV} / \mathrm{c}^{2}$ away from the signal region center for the untagged (tagged) channels. The sideband definitions for the five channels are reported at the end of this section.

The first step of the optimization is a study of the reconstructed-mass distribution of the five channels. Since data distributions differ from the MC ones, as shown in Appendix B, we carry on the optimization separately for data and MC events. For the MC optimization study we have used another cocktail, none of the ones listed in Sec. 4.1 and not independent from them. This choice was made in order to optimize the signal region definition for MC independently of the cocktails that we will use to test the simultaneous fit. In this way we have optimized for all the 4 cocktails instead of only one.

\subsubsection{Mass Fits}

The fits are performed in order to determine the $D^{0}$ mass peak positions and shapes for the five channels. We also use the extracted PDF to estimate the number of background candidates in the signal region. In these fits it is not possible distinguish the misreconstructed-charm events from the combinatorial ones, therefore we divide the events in only two categories: signal and background. The fit range is mode dependent in order to eliminate the reflection backgrounds and they are given in the caption of the Figures showing the fit results. We performed fits to data and to simulated events. Since in some cases the line shapes are different in data and MC (see Appendix B) different PDF forms and fit ranges are used in the MC fit. In the following we only report the results of the data fits. 
The basic functions used to perform the fits are:

- Gaussian Function

$$
G(x ; \mu, \sigma)=\frac{1}{\sqrt{2 \pi \sigma}} \exp \left[-\frac{(x-\mu)^{2}}{2 \sigma^{2}}\right]
$$

\section{- Crystal Ball Function}

$$
\begin{aligned}
& C B(x ; \bar{x}, \sigma, \alpha, n)= \\
& C_{\mathrm{CB}} \begin{cases}\exp \left(-\frac{(x-\bar{x})^{2}}{2 \sigma^{2}}\right) & \text { if } \frac{x-\bar{x}}{\sigma}>-\alpha \\
\left(\frac{n}{|\alpha|}\right)^{n} \frac{\exp \left(-\frac{1}{2} \alpha^{2}\right)}{\left(\frac{n}{|\alpha|}-|\alpha| \pm \frac{x-\bar{x}}{\sigma}\right)^{n}} & \text { otherwise, }\end{cases}
\end{aligned}
$$

and where the $+\operatorname{sign}$ is chosen for $\alpha \geq 0$, the - sign otherwise.

\section{- Chebyshev Polynomials}

The Chebyshev Polynomials are centered in the middle, and normalized over the fit range. We use the first order $\left(T_{1}(x)\right)$ and the second order $\left(T_{2}(x)\right)$ polynomials, defined as:

$$
\begin{aligned}
T_{1}\left(x ; c_{1}\right) & =C\left[1+c_{1} x\right] \\
T_{2}\left(x ; c_{1}, c_{2}\right) & =C\left[1+c_{1} x+c_{2}\left(2 x^{2}-1\right)\right],
\end{aligned}
$$

where $C$ is determined by the normalization requirement.

In case the events in the bin are Poisson distributed, the $\chi^{2}$ of the fit is given by [53]:

$$
\chi^{2}=\sum_{i}\left|\delta_{\mathrm{HF}}^{i}\right|^{2}=\sum_{i}\left[2\left(N_{i}^{\mathrm{pdf}}-N_{i}^{\mathrm{obs}}\right)+2 N_{i}^{\mathrm{obs}} \log \frac{N_{i}^{\mathrm{obs}}}{N_{i}^{\mathrm{pdf}}}\right]
$$

where the sum runs over the bins, $N_{i}^{\text {obs }}$ is the number of events in the $i-$ th bin and $N_{i}^{\text {pdf }}$ is the value of the PDF in that bin. In order to test the agreement between the PDF and the distribution we report below each plot the value of the $\delta_{\mathrm{HF}}^{i}$, positive if $N_{i}^{\text {obs }} \geq N_{i}^{\text {pdf }}$, negative otherwise.

\section{Tagged Channels Mass Fits}

For both the tagged $C P$-even modes we perform a simultaneous fits to the $D^{0}$ and $\bar{D}^{0}$ samples sharing the PDF parameters but allowing for different signal and background yields. The background is parameterized as a first order Chebyshev polynomial for both 
channels. The signal PDF for the $\pi^{+} \pi^{-}$channel consists of the sum of two gaussians $(G)$ while for the $K^{+} K^{-}$channel we add also a Crystal Ball function $(C B)$. For the tagged $K^{ \pm} \pi^{\mp}$ channel we parameterize the signal PDF with the sum of three Gaussian functions $(G)$ and the background PDF is a second order Chebyshev polynomial. Unlike the other two tagged channels, $D^{0}$ and $\bar{D}^{0}$ candidates are together in a unique sample. The results of the three fits are reported in Fig. 4.2 and the values of the extracted parameters in Appendix C.

\section{Untagged Channels Mass Fits}

For both the untagged channels the signal PDF consists of a sum of three Gaussian functions $(G)$ and the background $\mathrm{PDF}$ as a second order Chebyshev polynomial. In Figure 4.3 we report the result of the fits to data for both channels. The value of the extracted parameters can be found in Appendix C.

\subsubsection{Signal Region Optimization}

The most general parameterization of the signal region for a given channel is

$$
\left[m_{C}-\frac{w}{2}(1-\alpha), m_{C}+\frac{w}{2}(1+\alpha)\right]
$$

where $m_{C}$ is the signal-region center, $w$ is the total width, and $\alpha$ describes the asymmetry of the interval with respect to $m_{C}$. The signal region should be centered at the peak of the mass distribution, corresponding to the most probable value of the PDF. The other two free parameters allow one to define a signal region such that:

1. the number of events contained on the right side of the interval is the same as the number of events contained on the left side;

2. the error on the lifetime that depends on the number of signal and background candidates is minimized.

Under the hypothesis of a symmetric mass PDF and a linear correlation between the reconstructed mass and the proper time, the first constraint allows one to cancel the effect of the $t-m_{D^{0}}$ correlation since we integrate all the events over the mass range. This requirement sets the value of $\alpha$. The second constraint sets the width of the signal region, $w$.

From the extracted mass PDF we have computed the most probable value (MPV) and the right and left half-width-half-maximum (HWHM) values for the five channels. These properties are reported Tab. 4.3 for data and in Tab. 4.4 for MC.

In the MC sample the distance between the two more distant MPV is quite small, $65 \mathrm{keV} / \mathrm{c}^{2}$ while in data it is much larger, $340 \mathrm{keV} / c^{2}$. The $\mathrm{MC}$ distributions are also less asymmetric 

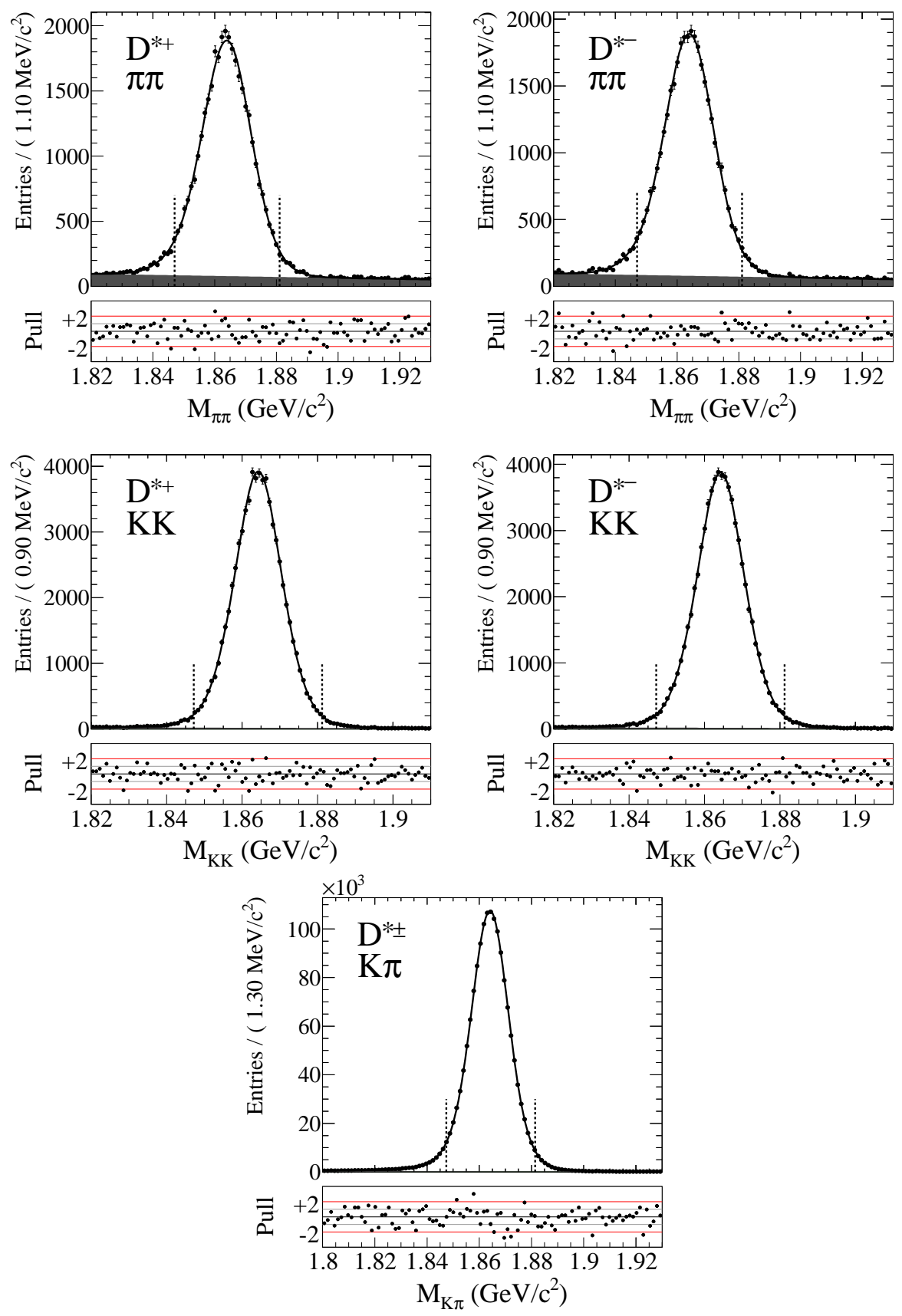

Figure 4.2: Fits to the tagged $\pi^{+} \pi^{-}$(top), $K^{+} K^{-}$(middle) and $K^{ \pm} \pi^{\mp}$ (bottom) mass distributions for data. The left (right) plots of the first two rows are the projection on $D^{0}\left(\bar{D}^{0}\right)$ events. The shaded regions are the background contributions. The vertical lines show the signal regions, defined in Sec. 4.3. The fit ranges are $1.82<m_{D^{0}}\left(\mathrm{GeV} / c^{2}\right)<1.93$ for the $\pi^{+} \pi^{-}$channel, $1.82<m_{D^{0}}\left(\mathrm{GeV} / c^{2}\right)<1.91$ for the $K^{+} K^{-}$channel, and $1.80<$ $m_{D^{0}}\left(\mathrm{GeV} / c^{2}\right)<1.93$ for the $K^{ \pm} \pi^{\mp}$ one. A cut of $0.1447<\Delta m\left(\mathrm{GeV} / c^{2}\right)<0.1463$ is applied. 

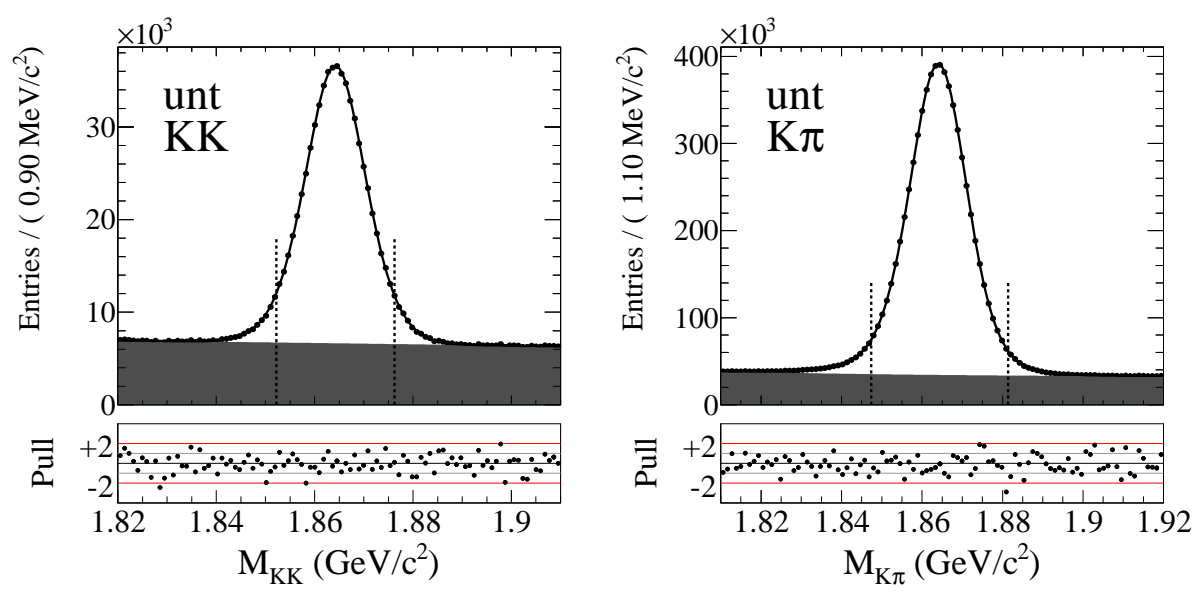

Figure 4.3: Fits to the untagged $K^{+} K^{-}$(left) and $K^{ \pm} \pi^{\mp}$ (right) mass distribution for data. The shaded regions are the background contributions. The vertical lines show the signal regions, defined in Sec. 4.3. The fit ranges are $1.82<m_{D^{0}}\left(\mathrm{GeV} / c^{2}\right)<1.91$ for the untagged $K^{+} K^{-}$channel and $1.8<m_{D^{0}}\left(\mathrm{GeV} / c^{2}\right)<1.93$ for the untagged $K^{ \pm} \pi^{\mp}$ channel.

and have a slightly better resolution than data. Focusing on data, we observe that the untagged channels have slightly better resolutions than the corresponding tagged ones. The difference in resolutions is dominated by the types of particles in the final states: the presence of pions degrades the resolution and also increase the asymmetry.

In the following we present the studies that allow one to define the signal region, namely the values of $m_{C}, \alpha$ and $w$ for the five channels.

\section{Determination of Signal Region Centers $m_{C}$}

The distance between the two more distant MPVs in data is considerable, $340 \mathrm{keV} / \mathrm{c}^{2}$, and we decided to subdivide the five channels in groups with similar MPVs. Observing Tab. 4.3 we note that the tagged and untagged $K^{+} K^{-}$channels have very similar MPV, as well as the tagged and untagged $K^{ \pm} \pi^{\mp}$ channels, while the $\pi^{+} \pi^{-}$is shifted to a slight lower value. Since the $\pi^{+} \pi^{-}$sample is the least important in terms of statistics, we decided to have two distinct centers, one for the $K^{+} K^{-}$channels (tagged and untagged) and another for the other three channels $\left(\pi^{+} \pi^{-}\right.$and tagged and untagged $\left.K^{ \pm} \pi^{\mp}\right)$. The values of the two centers are computed as weighted averages of the MPV of Tab. 4.3 using as weights the number of fitted signal events. We obtain:

- tagged and untagged $K^{+} K^{-}$channels: $m_{C}^{\mathrm{KK}}=1864.215 \mathrm{MeV} / c^{2}$

- tagged $\pi^{+} \pi^{-}$and tagged and untagged $K^{ \pm} \pi^{\mp}$ channels: $m_{C}^{\text {nonKK }}=1864.042 \mathrm{MeV} / c^{2}$

The choice of two rather than a common center is enforced by the fact that when choosing a center that is quite distant from the channel peak (greater than $\sim 150 \mathrm{keV} / c^{2}$ ) the 


\begin{tabular}{c|ccccc}
\hline \hline \multirow{2}{*}{ property $\left(\mathrm{MeV} / c^{2}\right)$} & \multicolumn{3}{|c}{ Tagged } & \multicolumn{2}{c}{ Untagged } \\
& $\pi^{+} \pi^{-}$ & $K^{+} K^{-}$ & $K^{ \pm} \pi^{\mp}$ & $K^{+} K^{-}$ & $K^{ \pm} \pi^{\mp}$ \\
\hline MPV & 1863.931 & 1864.272 & 1864.073 & 1864.200 & 1864.035 \\
left HWHM & 9.548 & 7.328 & 8.536 & 7.238 & 8.494 \\
right HWHM & 9.262 & 7.277 & 8.401 & 7.238 & 8.345 \\
\hline \hline
\end{tabular}

Table 4.3: Mass PDF properties extracted from the fit to data reported in Fig. 4.2 and Fig. 4.3.

\begin{tabular}{c|ccccc}
\hline \hline \multirow{2}{*}{ property $\left(\mathrm{MeV} / c^{2}\right)$} & \multicolumn{3}{|c}{ Tagged } & \multicolumn{2}{c}{ Untagged } \\
& $\pi^{+} \pi^{-}$ & $K^{+} K^{-}$ & $K^{ \pm} \pi^{\mp}$ & $K^{+} K^{-}$ & $K^{ \pm} \pi^{\mp}$ \\
\hline MPV & 1864.542 & 1864.502 & 1864.507 & 1864.477 & 1864.518 \\
left HWHM & 9.493 & 7.092 & 8.330 & 7.132 & 8.343 \\
right HWHM & 9.229 & 7.097 & 8.258 & 7.104 & 8.232 \\
\hline \hline
\end{tabular}

Table 4.4: Mass PDF properties extracted from the fit to MC events.

asymmetry changes significantly. On the other hand having many different centers complicates significantly the evaluation of the systematic error. Two centers seems a reasonable compromise.

For what concerns the MC events, the distance between the two more distant MPV is much smaller than data, $65 \mathrm{keV} / c^{2}$, therefore we decided to have a single value for $m_{C}$ for all the five channels for MC. The weighted average yields:

- all channels (in MC): $m_{C}^{\mathrm{KK}}=1864.514 \mathrm{MeV} / c^{2}$

The value obtained here agrees with the generated mass in MC and it is the same used in the previous analyses.

\section{Determination of Signal Region Asymmetry $\alpha$}

Requiring that the signal region contains an equal number of signal events on the right and on the left side of the center $m_{C}$ we found that the value of the asymmetry parameter $\alpha$ is smaller than $7 \%$ for all the channels for widths from $0 \mathrm{MeV} / c^{2}$ to $35 \mathrm{MeV} / c^{2}$ (see Appendix D.1). We therefore decide to fix $\alpha=0$ for all the channels, for data and MC.

\section{Determination of Signal Region Width $w$}

In the previous analyses the statistical error was the dominant one. For this reason we have decided to optimize the signal region width minimizing the statistical error on the lifetimes for the five channels, but taking into account the systematic impact of the background. Therefore, as first step we minimize the statistical error, but then we evaluate 
the systematic error related to the background in the untagged $K^{+} K^{-}$channel, the one with more background.

In order to choose the width of the signal region minimizing the statistical error on the lifetime, we have performed Toy MC studies: we have generated events from the signalchannel proper time PDF (presented later in Sec. 5.1.1), varying the number of signal and background events according to the integral of the PDFs and evaluating the error on the lifetime. We have repeated this procedure for the five channels separately. The results are reported in Appendix D.2. This study shows that, from the point of view of the statistical error, the signal region width can be the same for all the channels because the relative difference between the statistical errors at different signal region widths is smaller than $10 \%$. The study suggests to use large signal regions, $34 \mathrm{MeV} / c^{2}$ wide for the tagged but also for the untagged events. The only concern about having such large signal regions for the untagged samples is that the background levels becomes higher than the ones registered in the previous analyses where the width was $20 \mathrm{MeV} / \mathrm{c}^{2}$. The systematic impact of the background in the previous untagged analysis was basically dominated by the untagged $K^{+} K^{-}$channel, as expected. We therefore decided to evaluate the impact of the background for this channel and minimize the total, statistical plus systematic, error. The evaluation of the systematic error was done applying the systematic variations used in the previous analyses to data in a blind fit and reporting the variations of the lifetimes as a function of the signal region width. The results of the study are reported in Appendix D.2 and indicate that the signal region width that yields the smallest total error for the untagged $K^{+} K^{-}$lifetime is $24 \mathrm{MeV} / c^{2}$.

The signal regions are therefore $24 \mathrm{MeV} / c^{2}$ wide for the untagged $K^{+} K^{-}$channel and $34 \mathrm{MeV} / c^{2}$ wide for the other channels. The wider signal region applies also to the untagged $K^{ \pm} \pi^{\mp}$ channel since in the previous untagged analysis the systematic errors associated with the background in this channel were one order of magnitude smaller than the same errors evaluated on the $K^{+} K^{-}$.

Repeating the same studies on the MC sample we found that the optimal widths are $20 \mathrm{MeV} / c^{2}$ wide for the untagged $K^{+} K^{-}$channel and $30 \mathrm{MeV} / c^{2}$ wide for the other channels.

\section{Final Results}

The definition of the signal regions is reported in Tab. 4.5 (Tab. 4.6) for data (MC).

The low and high sidebands are defined in terms of their distance from the center of the 


\begin{tabular}{c|ccccc}
\hline \hline \multirow{2}{*}{ parameters } & \multicolumn{3}{|c}{ Tagged } & \multicolumn{2}{c}{ Untagged } \\
& $\pi^{+} \pi^{-}$ & $K^{+} K^{-}$ & $K^{ \pm} \pi^{\mp}$ & $K^{+} K^{-}$ & $K^{ \pm} \pi^{\mp}$ \\
\hline$m_{C}\left(\mathrm{MeV} / c^{2}\right)$ & 1864.042 & 1864.215 & 1864.042 & 1864.215 & 1864.042 \\
$w\left(\mathrm{MeV} / c^{2}\right)$ & 34 & 34 & 34 & 24 & 34 \\
$\alpha$ & 0 & 0 & 0 & 0 & 0 \\
\hline$m_{\min }\left(\mathrm{MeV} / c^{2}\right)$ & 1847.042 & 1847.215 & 1847.042 & 1852.215 & 1847.042 \\
$m_{\max }\left(\mathrm{MeV} / c^{2}\right)$ & 1881.042 & 1881.215 & 1881.042 & 1876.215 & 1881.042 \\
\hline \hline
\end{tabular}

Table 4.5: Signal region parameters and definition for data.

\begin{tabular}{c|ccccc}
\hline \hline \multirow{2}{*}{ parameters } & \multicolumn{3}{|c}{ Tagged } & \multicolumn{2}{c}{ Untagged } \\
& $\pi^{+} \pi^{-}$ & $K^{+} K^{-}$ & $K^{ \pm} \pi^{\mp}$ & $K^{+} K^{-}$ & $K^{ \pm} \pi^{\mp}$ \\
\hline$m_{C}\left(\mathrm{MeV} / c^{2}\right)$ & 1864.514 & 1864.514 & 1864.514 & 1864.514 & 1864.514 \\
$w\left(\mathrm{MeV} / c^{2}\right)$ & 30 & 30 & 30 & 20 & 30 \\
$\alpha$ & 0 & 0 & 0 & 0 & 0 \\
\hline$m_{\min }\left(\mathrm{MeV} / c^{2}\right)$ & 1849.514 & 1849.514 & 1849.514 & 1849.514 & 1849.514 \\
$m_{\max }\left(\mathrm{MeV} / c^{2}\right)$ & 1879.514 & 1879.514 & 1879.514 & 1874.514 & 1879.514 \\
\hline \hline
\end{tabular}

Table 4.6: Signal region parameters and definition for MC.

signal region, $d$, and the their width, $w S B$ :

$$
\begin{array}{crl}
\text { low sideband: } \quad m_{\min } & =m_{C}-d-w S B / 2 \\
& m_{\max }=m_{C}-d+w S B / 2 \\
\text { high sideband: } \quad m_{\min } & =m_{C}+d-w S B / 2 \\
m_{\max } & =m_{C}+d+w S B / 2 .
\end{array}
$$

The values of $d$ and $w S B$ are reported in Tab. 4.7 and are the same for data and MC.

\begin{tabular}{r|ccccc}
\hline \hline \multirow{2}{*}{ parameters } & \multicolumn{3}{|c}{ Tagged } & \multicolumn{3}{c}{ Untagged } \\
& $\pi^{+} \pi^{-}$ & $K^{+} K^{-}$ & $K^{ \pm} \pi^{\mp}$ & $K^{+} K^{-}$ & $K^{ \pm} \pi^{\mp}$ \\
\hline$w S B\left(\mathrm{MeV} / c^{2}\right)$ & 20 & 20 & 20 & 20 & 20 \\
$d\left(\mathrm{MeV} / c^{2}\right)$ & 35.5 & 35.5 & 35.5 & 44.5 & 44.5 \\
\hline \hline
\end{tabular}

Table 4.7: Sidebands parameters $d$ and $w S B$ for data and MC.

\subsection{Expected Number of Signal and Background Candi- dates}

In the final fit we fix the expected misreconstructed-charm and combinatorial events in the signal region. We need therefore to have a prediction for the yields of the background categories in the signal region. 
For what concerns the misreconstructed-charm category, we estimate the number of events belonging to this category using the MC dataset, namely bigCharm. The definition of the signal region is different in data and MC, we assume that the mass distribution in MC is shifted with respect to data, therefore we use the central value $\left(m_{C}\right)$ estimated on $\mathrm{MC}$ but the width $(w)$ estimated on data (see Tab. 4.5 and 4.6). This treatment of the signal region is also applied in the extraction of the combinatorial yields.

In order to reduce our dependence on MC, we extract the expected number of combinatorial events in the signal region from the mass fits. Looking at the comparison between the fitted and the true yields in the MC fits to the mass distribution (see Tab. 4.8), we extract a correction factor $\left(s_{m}\right)$ defined as "truth background yields/fitted background yields" and apply it to the background yields extracted from the data mass fits. Then we subtract the misreconstructed-charm yields obtained from MC and we obtain the combinatorial yields in the signal region. The systematic error associated with the choice of the value of the scale factor is discussed in Chapter 6 .

\begin{tabular}{c|ccccc}
\hline \hline \multirow{2}{*}{$\mathrm{MC}$} & \multicolumn{4}{|c}{ Tagged } & \multicolumn{3}{c}{ Untagged } \\
& $\pi^{+} \pi^{-}$ & $K^{+} K^{-}$ & $K^{ \pm} \pi^{\mp}$ & $K^{+} K^{-}$ & $K^{ \pm} \pi^{\mp}$ \\
\hline & \multicolumn{5}{|c}{ Truth Yields } \\
\hline Signal & 64583 & 149035 & 1488310 & 481907 & 5428393 \\
Background & 2954 & 581 & 2312 & 140721 & 814080 \\
\hline \hline & \multicolumn{5}{c}{ Fit Yields } \\
\hline Signal & 62618 & 151203 & 1505833 & 489554 & 5543697 \\
Background & 3821 & 967 & 5061 & 137497 & 825378 \\
\hline \hline & \multicolumn{5}{|c}{ Background Scale Factor } \\
\hline$s_{m}$ & 0.773 & 0.601 & 0.457 & - & 0.986 \\
\hline \hline
\end{tabular}

Table 4.8: MC Truth and fitted yields of signal and background in the signal region for the five channels in the MC cocktail (the one used in the optimization of the signal region). The scale factor used to scale the background yields in the signal region estimated with $\mathrm{MC}$ is also reported. The scale factor for the untagged $K^{+} K^{-}$channel has not been used, as explained in the text.

In Tab. 4.9 we report the result of this procedure that has been demonstrated to work for all channels except the untagged $K^{+} K^{-}$one.

While performing fits to the $\left(t, \sigma_{t}\right)$ distribution in data, we realized that the prediction of the expected number of background events for the untagged $K^{+} K^{-}$channel obtained with the mass fits was not sufficiently well determined and caused the offset of the signal resolution function for this channel to not be compatible with the offsets of the other channels. We therefore adopted a different strategy for this channel: we extract the number of combinatorial events directly in the simultaneous fit and not make use of the background events extracted from the mass fits.

The estimated purity, defined as the ratio of the number of signal events divided by the 


\begin{tabular}{c|ccccc}
\hline \hline data & \multicolumn{4}{|c}{ Tagged } & \multicolumn{3}{c}{ Untagged } \\
& $\pi^{+} \pi^{-}$ & $K^{+} K^{-}$ & $K^{ \pm} \pi^{\mp}$ & $K^{+} K^{-}$ & $K^{ \pm} \pi^{\mp}$ \\
\hline & \multicolumn{5}{c}{ Fit Yields } \\
\hline Signal & 63279 & 138882 & 1430695 & 485633 & 5602261 \\
Background & 4860 & 1435 & 7638 & 177018 & 1064094 \\
\hline \hline & \multicolumn{5}{c}{ Rescaled Yields } \\
\hline Background & 3760 & 862 & 3491 & - & 1049528 \\
\hline \hline & \multicolumn{5}{c}{ Background Composition } \\
\hline misrec.-charm & 97 & 310 & 642 & 5478 & 4645 \\
combinatorial & 3663 & 553 & 2849 & 164970 & 1044552 \\
\hline \hline
\end{tabular}

Table 4.9: Fitted yields of signal and background in the signal region for the five channels in data, corresponding to the fits shown in Fig. 4.2 and Fig. 4.3. The rescaled background yields are obtained using the scale factors reported in Tab. 4.8. The scale factor for the untagged $K^{+} K^{-}$channel has not been used, as explained in the text, here we anticipate the extracted value from the final mixing fit.

total number of events, of each of the five channels for the events used in the fit are reported in Tab. 4.10 for data and MC. The estimated purities in data are slightly lower

\begin{tabular}{c|ccccc}
\hline \hline \multirow{2}{*}{ purity } & \multicolumn{3}{|c}{ Tagged } & \multicolumn{3}{c}{ Untagged } \\
& $\pi^{+} \pi^{-}$ & $K^{+} K^{-}$ & $K^{ \pm} \pi^{\mp}$ & $K^{+} K^{-}$ & $K^{ \pm} \pi^{\mp}$ \\
\hline MC & $95.6 \%$ & $99.6 \%$ & $99.8 \%$ & $77 \%$ & $87 \%$ \\
data & $92.9 \%$ & $99.0 \%$ & $99.5 \%$ & $73 \%$ & $84 \%$ \\
\hline \hline
\end{tabular}

Table 4.10: Purities of the five channels for the events used in the fit, estimated in $\mathrm{MC}$ and in data. Note that the definition of the signal region in $\mathrm{MC}$ differ from the data definition (see Sec. 4.3.2).

than in MC; this is a consequence of the wider signal region in data. However, the number of signal events in data estimated from the mass fits, although in a wider signal region, is not higher than the MC estimation. 


\section{Chapter 5}

\section{Measurement of $y_{C P}$ and $\Delta Y$}

In this chapter we provide a description of the events in the signal region, classified in the three categories: signal, misreconstructed-charm and combinatorial. We show the studies on the proper time resolution of the signal candidates, and the studies of the background events, in particular the composition of the misreconstructed-charm background, and the relevant distributions of the events in the mass sidebands. We present the signal and background PDFs for the five channels. Finally we report the validations of the fit on simulated events and the results of the nominal fit to data.

\subsection{Characterization of the Signal Events}

Signal candidates are defined as correctly reconstructed $D^{0}$ decays. This category includes events with radiative photons or decays in flight of kaons or pions. Concerning the tagged channels, the $D^{0}$ candidates combined with an incorrectly reconstructed slow pion are also included, with a dedicated component of the PDF.

In Fig. 5.1 we report the mass, $\Delta m$ and proper time error distributions for the truthmatched signal events for the tagged $K^{+} K^{-}$channel. The events in this category are
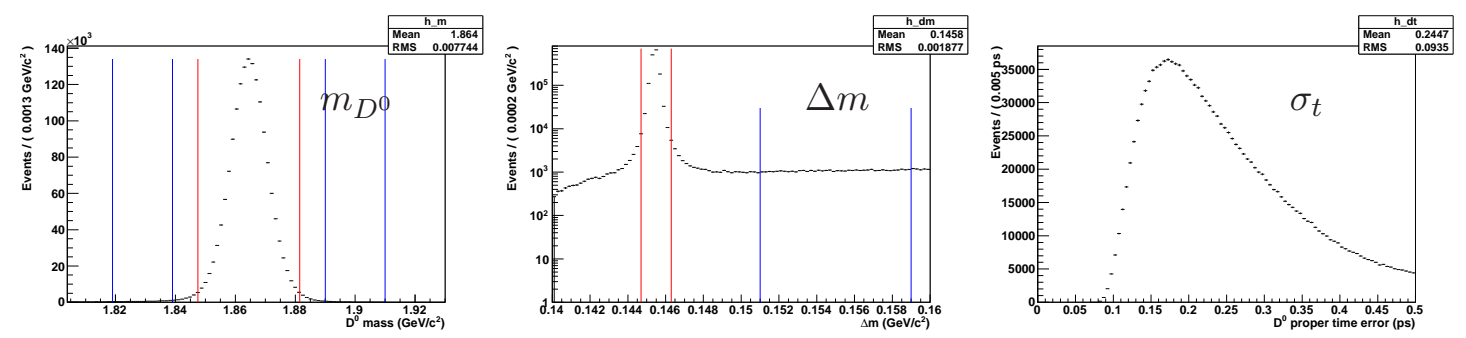

Figure 5.1: Signal truth-matched distributions for the tagged $K^{+} K^{-}$events in the signal region: reconstructed mass (left), $\Delta m$ (center), and proper time error (right). The red lines indicate the signal region, the blue lines indicate the sideband regions. The proper time error distribution include only events in the signal region. 
peaking in mass and, for the tagged channels, also in $\Delta m$. The mean proper time error estimated from simulated events is $\sim 0.24 \mathrm{ps}$, around $60 \%$ of the $D^{0}$ lifetime. In Appendix E.1 we report the relevant distributions for the five channels.

Before performing any fit to the reconstructed proper time, we check that the signal efficiency is independent of the proper time calculated using MC truth information, the "true proper time", $t_{\text {true }}$. We fit the true proper time distribution for each of the 9 independent signal MC datasets, splitting the $D^{0}$ from the $\bar{D}^{0}$ events for the tagged channels. In the fits we remove the cut on the reconstructed proper time. The fits to oneSignal1 are reported in Fig. 5.2 and Fig. 5.3, a summary of the fitted lifetimes is in Tab. 5.1 and Tab. 5.2.

\begin{tabular}{l|ccc}
\hline \hline & & Tagged & \\
& $\pi^{+} \pi^{-}$ & $K^{+} K^{-}$ & $K^{ \pm} \pi^{\mp}$ \\
\hline$D^{0}$ average lifetime (fs) & $411.41 \pm 0.74$ & $411.18 \pm 0.49$ & $411.72 \pm 0.16$ \\
$\bar{D}^{0}$ average lifetime (fs) & $413.00 \pm 0.75$ & $411.92 \pm 0.48$ & $411.75 \pm 0.16$ \\
\hline average (fs) & $412.20 \pm 0.53$ & $411.56 \pm 0.34$ & $411.73 \pm 0.11$ \\
\hline \hline
\end{tabular}

Table 5.1: Averages of the $D^{0}$ and $\bar{D}^{0}$ lifetimes extracted from the 9 independent signal $\mathrm{MC}$ datasets for the tagged channels. The lifetime input to the MC is $411.67 \mathrm{fs}$.

\begin{tabular}{l|cc}
\hline \hline & \multicolumn{2}{|c}{ Untagged } \\
& $K^{+} K^{-}$ & $K^{ \pm} \pi^{\mp}$ \\
\hline average lifetime (fs) & $412.03 \pm 0.19$ & $411.96 \pm 0.06$ \\
\hline \hline
\end{tabular}

Table 5.2: Averages of the $D$ lifetime extracted from the 9 independent signal MC datasets for the untagged channels. The lifetime input to the $\mathrm{MC}$ is $411.67 \mathrm{fs}$.

The tagged $D^{0}$ and $\bar{D}^{0}$ extracted lifetimes are compatible with the generated one within one standard deviation. The lifetimes extracted from the untagged channels are slightly higher than the generated one. However, the simultaneous fit to reconstructed proper time distribution, considering signal-only events, demonstrates that there is no bias on the observables $y_{C P}$ and $\Delta Y$, as shown later in Sec. 5.1.2.

\section{Proper Time Resolution}

The resolution is defined as $\left(t-t_{\text {true }}\right)$, where $t_{\text {true }}$ is the generated proper time. In Fig. 5.4 we report the resolution distributions for the five channels, and in Tab. 5.3 the mean and the RMS of the distributions, which are quite different for the different channels.

Since we want to perform a simultaneous fit of the five channels, sharing as much as possible of the resolution function, we consider the proper time error and study the resolution pull, defined as $\left(t-t_{\text {true }}\right) / \sigma_{t}$. In Fig. 5.5 we report the resolution-pull distributions for the five 

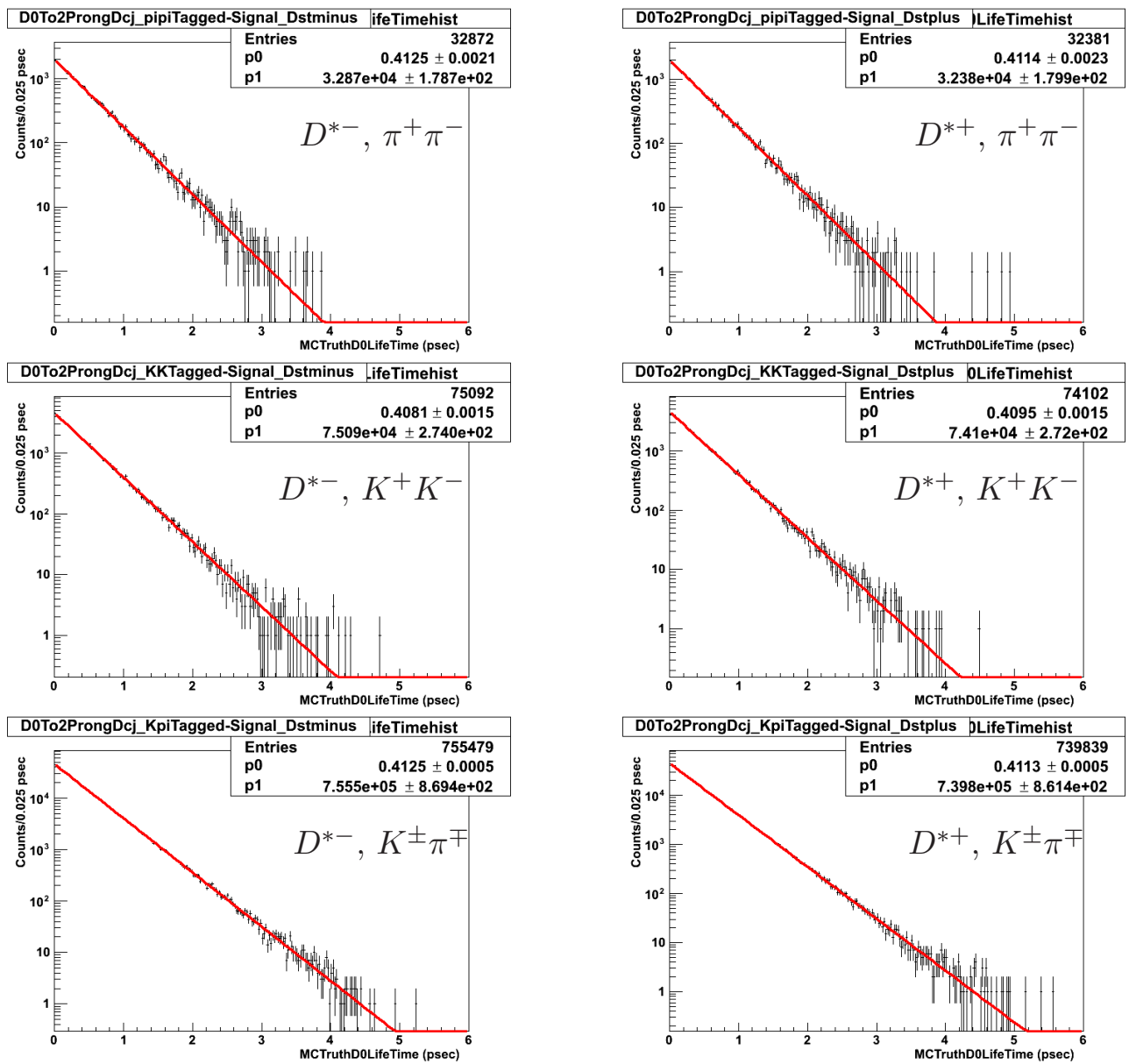

Figure 5.2: Binned fit to the true proper time $t_{\text {true }}$ for $D^{0}$ (right) and $\bar{D}^{0}$ (left) tagged candidates. These results are an example from the signal-only MC dataset oneSignal1. In this study the $D^{0}$ and $\bar{D}^{0}$ decays in the $K^{ \pm} \pi^{\mp}$ channels have been separated.
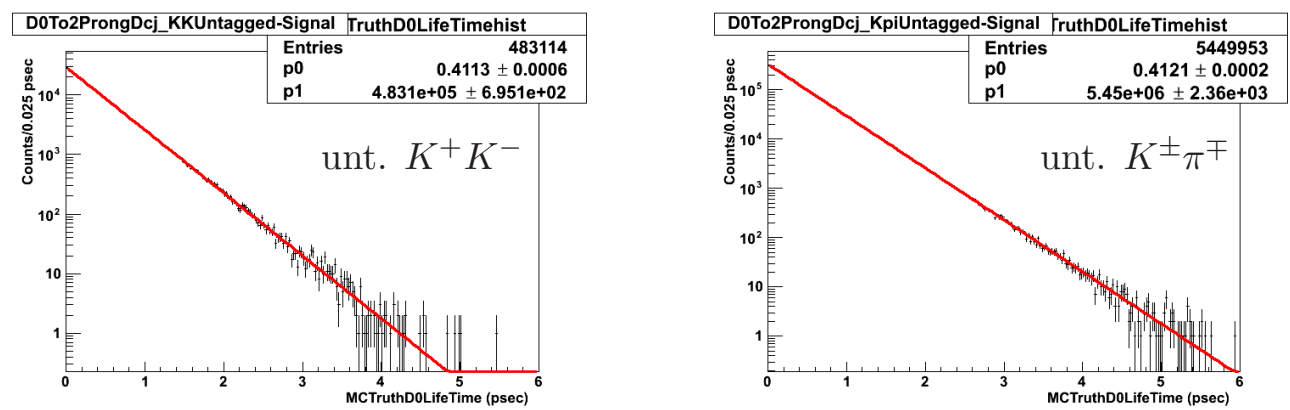

Figure 5.3: Binned fit to the true proper time $t_{\text {true }}$ for untagged channels. These results are an example from the signal-only MC dataset oneSignal1.

channels estimated on the simulated events, and in Tab. 5.4 the mean and the RMS of the distributions.

The RMS of the untagged $K^{+} K^{-}$channel is $\sim 10 \%$ lower than the other channels because the signal region is $30 \%$ narrower for that channel. There are also slight differences among 

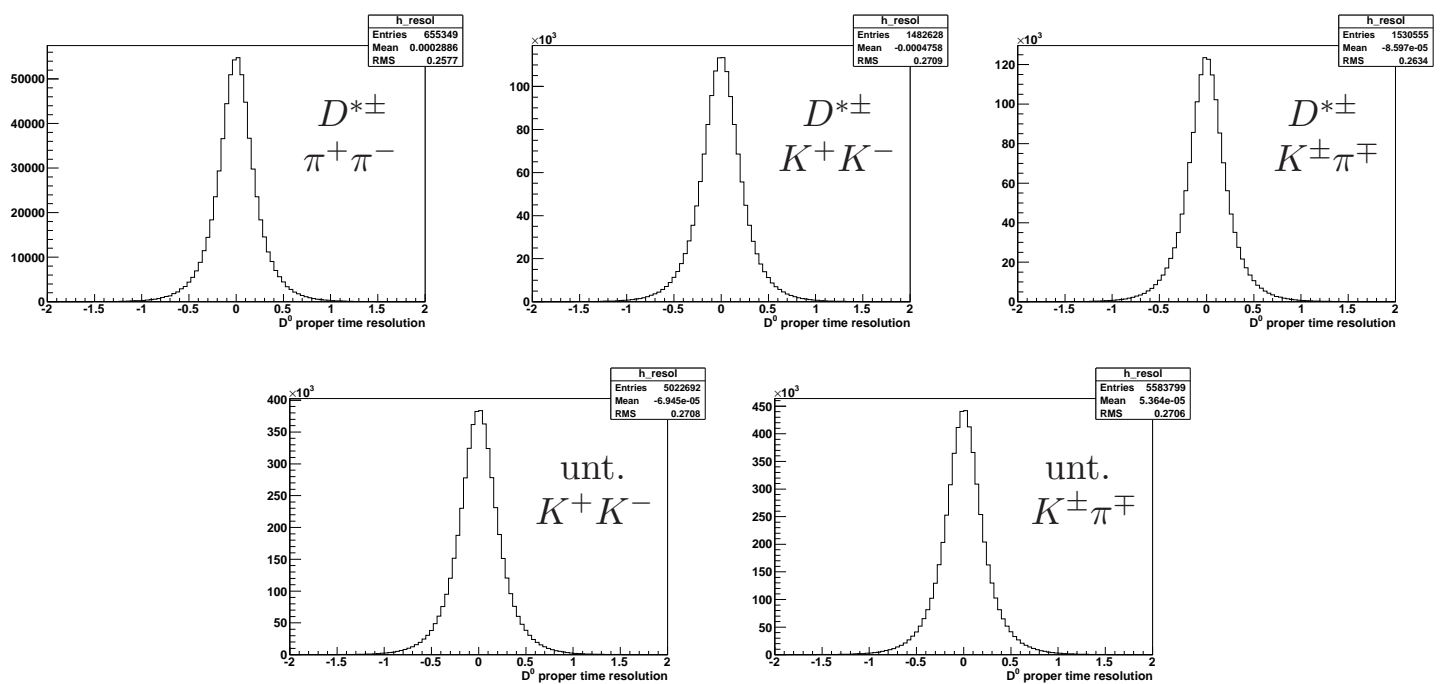

Figure 5.4: Distribution of the resolution $t-t_{\text {true }}$ (in ps) for signal events in the signal region for the five channels, estimated on the bigSignal MC dataset except for the $K^{ \pm} \pi^{\mp}$ channels, for which we use one cocktail.
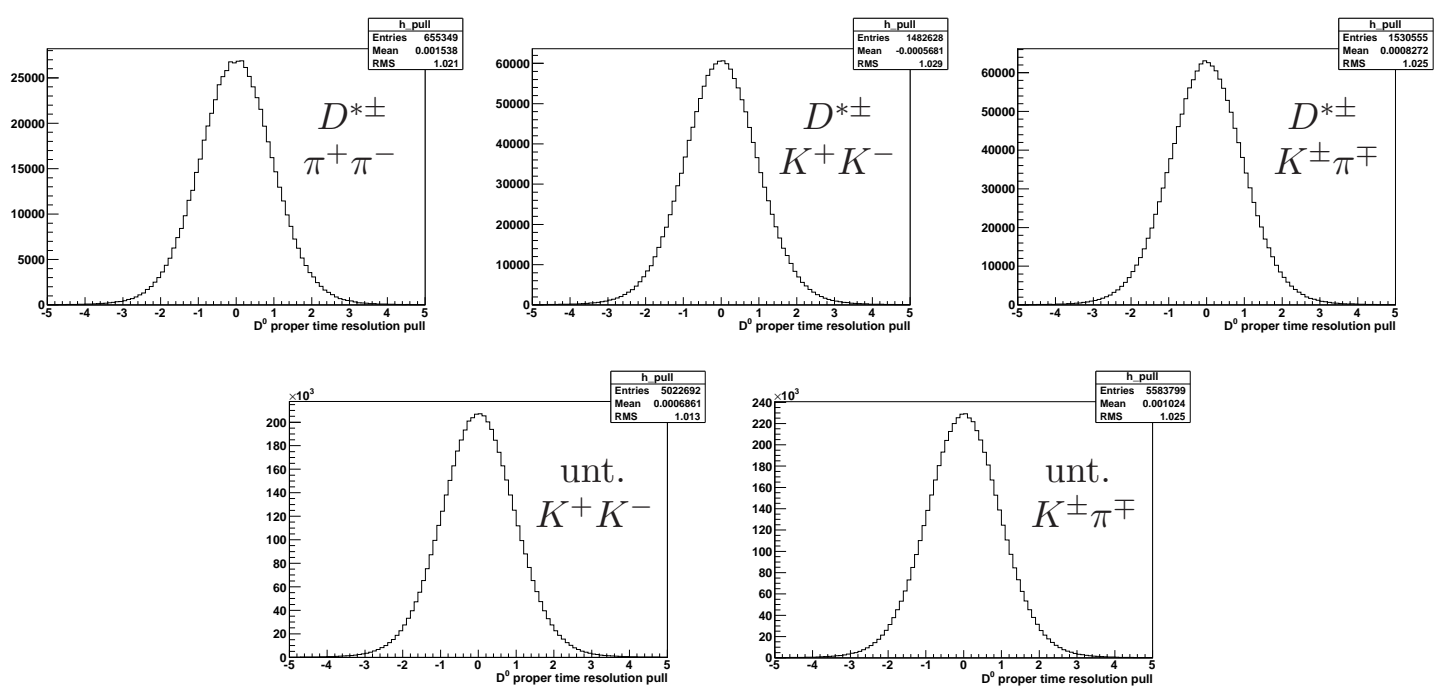

Figure 5.5: Distribution of the resolution pull $\left(t-t_{\text {true }}\right) / \sigma_{t}$ for signal events in the signal region for the five channels, estimated on the bigSignal MC dataset except for the $K^{ \pm} \pi^{\mp}$ channels, for which we use one cocktail. 


\begin{tabular}{l|ccccc}
\hline \hline & \multicolumn{3}{|c}{ Tagged } & \multicolumn{2}{c}{ Untagged } \\
& $\pi^{+} \pi^{-}$ & $K^{+} K^{-}$ & $K^{ \pm} \pi^{\mp}$ & $K^{+} K^{-}$ & $K^{ \pm} \pi^{\mp}$ \\
\hline mean (fs) & 0.29 & -0.48 & -0.09 & -0.07 & 0.05 \\
RMS (fs) & 258 & 271 & 263 & 271 & 271 \\
\hline \hline
\end{tabular}

Table 5.3: Mean and RMS of the resolution distributions reported in Fig. 5.4, estimated on the simulated signal events.

\begin{tabular}{l|ccccc}
\hline \hline & \multicolumn{3}{|c}{ Tagged } & \multicolumn{3}{c}{ Untagged } \\
& $\pi^{+} \pi^{-}$ & $K^{+} K^{-}$ & $K^{ \pm} \pi^{\mp}$ & $K^{+} K^{-}$ & $K^{ \pm} \pi^{\mp}$ \\
\hline mean $/ 10^{-3}$ & 1.54 & -0.57 & 0.83 & 0.69 & 1.02 \\
RMS & 1.021 & 1.029 & 1.025 & 1.013 & 1.025 \\
\hline \hline
\end{tabular}

Table 5.4: Mean and RMS of the resolution-pull distributions reported in Fig. 5.5, estimated on the simulated signal events.

the other channels, up to around $2 \%$. This difference among the channels is taken into account in the signal PDF introducing scale factors for the proper time error. The mean of the distributions are compatible with zero within $0.15 \%$. In the nominal fit we assume that the offset of the signal resolution function is the same for the five channels. This study demonstrates that the offset is expected to be near zero for the simulated events, while in data there are effects, much as the vertex detector misalignment, that can result in a non-zero offset.

Since we make use of the per-event error $\sigma_{t}$ in the signal PDF, in order to avoid biases [54] on the extracted lifetimes, we multiply each signal $\operatorname{PDF} \mathcal{P}\left(t, \sigma_{t}\right)$ by $\mathcal{H}\left(\sigma_{t}\right)$, the proper time error PDF of that mode, i.e. the probability for having a given error $\sigma_{t}$. The product $\mathcal{P}\left(t, \sigma_{t}\right) \cdot \mathcal{H}\left(\sigma_{t}\right)$ is a properly normalized two-dimensional PDF. The $\sigma_{t}$ PDFs are one dimensional histograms, and they are discussed later in Sec. 5.4. This is done not only for the signal PDFs but also for the background PDFs that include the per-event error $\sigma_{t}$.

\subsubsection{Parameterization}

The signal PDF is a simultaneous PDF with seven components, one for each of the decays reported in Eqs. (4.1) to (4.7). The basic PDF for each of the seven modes is described in the following.

In previous $B A B A R$ mixing analyses $[17,50,51]$ it was shown that the proper time resolution function can be described as a sum of three Gaussians. Each Gaussian uses per-event proper time errors, $\sigma_{t}$, obtained from the vertex fit of the $D^{0}$ decay tree ${ }^{1}$. Each Gaussian is assigned its own scaling factor $s_{i}$ for the proper time error. To describe the proper time

\footnotetext{
${ }^{1}$ this is also true for the tagged channels: we do not use the additional information from the slow pion in order to get similar resolutions as the untagged candidates.
} 
distribution the resolution function is convolved with an exponential. The PDF for an exponential convolved with a single Gaussian is:

$$
\mathcal{D}\left(t, \sigma_{t} ; s, t_{0}, \tau\right)=C_{\sigma_{t}} \int \exp \left(-t_{\text {true }} / \tau\right) \exp \left(-\frac{\left(t-t_{\text {true }}+t_{0}\right)^{2}}{2\left(s \cdot \sigma_{t}\right)^{2}}\right) d t_{\text {true }}
$$

where the normalization coefficient $C_{\sigma_{t}}$ is chosen such that

$$
\int \mathcal{D}\left(t, \sigma_{t} ; s, t_{0}, \tau\right) d t=1 \quad \text { for all } \sigma_{t}
$$

This is known as a conditional $\mathrm{PDF}^{2}$. With this definition, the product $\mathcal{D}\left(t, \sigma_{t} ; s, t_{0}, \tau\right) \cdot \mathcal{H}\left(\sigma_{t}\right)$ is a properly normalized two-dimensional PDF, where $\mathcal{H}\left(\sigma_{t}\right)$ is the the proper time error PDF, discussed later in Sec. 5.4.

The proper time signal PDF used for each of the seven modes is the sum of three such functions with a common offset $t_{0}$, but different scaling factors, $s_{1}, s_{2}, s_{3}$ :

$$
\begin{aligned}
\mathcal{R}\left(t, \sigma_{t} ; s_{i}, t_{0}, \tau\right) & =f_{t 1} \mathcal{D}\left(t, \sigma_{t} ; s_{1}, t_{0}, \tau\right) \\
& +\left(1-f_{t 1}\right)\left[f_{t 2} \mathcal{D}\left(t, \sigma_{t} ; s_{2}, t_{0}, \tau\right)\right. \\
& \left.+\left(1-f_{t 2}\right) \mathcal{D}\left(t, \sigma_{t} ; s_{3}, t_{0}, \tau\right)\right]
\end{aligned}
$$

where $s_{i}=\left(s_{1}, s_{2}, s_{3}\right)$ represents the three scaling factors. The scaling factors, the offset, the fractions $f_{t 1}, f_{t 2}$, along with the lifetime parameter are variable parameters in the fit. In the different signal channels $\tau$ will be identified with $\tau^{+}, \bar{\tau}^{+}$, or $\tau_{K \pi}$.

\section{Mistagged $D^{0}$ Candidates}

In the tagged channels we include the $D^{0}$ candidates combined with a misidentified slow pion in the signal category. Roughly half of the misidentified slow pions will have the wrong charge, and the $D^{0}$ candidates will be assigned the opposite flavor. Therefore, in the PDF of the tagged $C P$-even modes the mistagged signal events need to be described separately from the correctly tagged events. The difference between the correctly tagged and the mistagged $D^{0}$ candidates is either that the reconstructed slow pion track does not correspond to the true slow pion, or that the charge of the track is wrongly measured. The proper time resolution and lifetime for these events is therefore expected be the same as for correctly tagged $D^{0}$ candidates. This assumption has been tested in the previous tagged analysis [50].

Hence, for the tagged $K^{+} K^{-}$and $\pi^{+} \pi^{-}$modes we use a signal PDF that takes into account the fact that the mistagged signal events are described by the effective lifetime

\footnotetext{
${ }^{2}$ See Chapter 6 of Ref. [55] for additional discussion.
} 
associated with the opposite flavor:

$$
\begin{aligned}
& \mathcal{P}_{h h}^{D^{*+}}\left(t, \sigma_{t} ; \tau^{+}, \bar{\tau}^{+}\right)=\left(1-f_{\text {tag }}^{+}\right) \mathcal{R}\left(t, \sigma_{t} ; s_{i}, t_{0}, \tau^{+}\right)+f_{\text {tag }}^{+} \mathcal{R}\left(t, \sigma_{t} ; s_{i}, t_{0}, \bar{\tau}^{+}\right) \\
& \mathcal{P}_{h h}^{D^{*-}}\left(t, \sigma_{t} ; \bar{\tau}^{+}, \tau^{+}\right)=\left(1-f_{\text {tag }}^{-}\right) \mathcal{R}\left(t, \sigma_{t} ; s_{i}, t_{0}, \bar{\tau}^{+}\right)+f_{\text {tag }}^{-} \mathcal{R}\left(t, \sigma_{t} ; s_{i}, t_{0}, \tau^{+}\right)
\end{aligned}
$$

with $h=K, \pi$, and $f_{\text {tag }}^{ \pm}$representing the fraction of mistagged signal events. The fraction of mistagged events has been evaluated using bigSignal MC dataset, as half of the fraction of events with a misidentified slow pion. It is fixed to $f_{\text {tag }}^{ \pm}=0.2 \%$ in the nominal fit and varied as part of the systematics.

\section{$D^{0}$ Content of the Untagged $K^{+} K^{-}$Channel}

The signal candidates of the untagged $K^{+} K^{-}$channel have unknown flavor, each candidate could be a $D^{0}$ or a $\bar{D}^{0}$. To be able to describe the proper time distribution of the candidates in this channel with the effective lifetimes $\tau^{+}$and $\bar{\tau}^{+}$, we need to assume a certain fraction of $D^{0}$ over the total number of candidates. We assume that the sample is composed half of $D^{0}$ and half of $\bar{D}^{0}$, defining the fraction of $D^{0}, f_{D^{0}}=0.5$. We associate a systematic error to this assumption. The PDF for this mode is therefore:

$$
\mathcal{P}_{K K}^{\text {unt }}\left(t, \sigma_{t} ; \bar{\tau}^{+}, \tau^{+}\right)=\left(1-f_{D^{0}}\right) \mathcal{R}\left(t, \sigma_{t} ; s_{i}, t_{0}, \bar{\tau}^{+}\right)+f_{D^{0}} \mathcal{R}\left(t, \sigma_{t} ; s_{i}, t_{0}, \tau^{+}\right)
$$

\section{Sharing the Proper Time Resolution Model Parameters}

In order to reduce the uncertainty on the measured lifetimes we use a common resolution function for all the tagged and untagged modes, which is allowed if the proper time resolution pulls for the five channels are basically the same. In case they differ, additional parameters can be used in order to describe the differences. To obtain resolutions as similar as possible for the tagged and untagged channels, we compute the tagged $D^{0}$ candidates proper time and uncertainty using the same information available for the untagged $D^{0}$ candidates. In particular, in the vertex fit, we use the same constraints as for the untagged case: a $D^{0}$ vertex constraint and a beam constraint on the $D^{0}$ momentum. The additional information from the slow pion is not employed.

Although $t$ and $\sigma_{t}$ are computed using the same information for all decays, there may be residual differences in the resolution among the channels, for instance due to differences in reconstruction between kaons and pions. We therefore do not set the resolution function for $K^{+} K^{-}$and $\pi^{+} \pi^{-}$to be exactly the same as in the $K^{ \pm} \pi^{\mp}$ final state. Instead we introduce the final-state-dependent scale factors $S_{X}=\left\{S_{K K}, S_{\pi \pi}, S_{K \pi}=1\right\}$, used to re-scale the shared Gaussian scaling factors $s_{i}$. We also have observed differences in the spectra of the $D^{0}$ momentum in the center of mass between the tagged and the untagged channels for a given final state. This difference may result in slightly different resolutions. 
Therefore we introduce an additional scale factor, $S_{Y}^{\prime}=\left\{S_{\mathrm{tag}}^{\prime}, S_{\mathrm{unt}}^{\prime}=1\right\}$ to take into account this effect.

The single-mode proper time signal PDF of Eq. (5.3) therefore becomes ${ }^{3}$ :

$$
\begin{aligned}
\mathcal{R}_{X}^{Y}\left(t, \sigma_{t} ; S_{X} S_{Y}^{\prime} s_{i}, t_{0}, \tau\right) & =f_{t 1} \mathcal{D}\left(t, \sigma_{t} ; S_{Y}^{\prime} S_{X} s_{1}, t_{0}, \tau\right) \\
& +\left(1-f_{t 1}\right)\left[f_{t 2} \mathcal{D}\left(t, \sigma_{t} ; S_{Y}^{\prime} S_{X} s_{2}, t_{0}, \tau\right)\right. \\
& \left.+\left(1-f_{t 2}\right) \mathcal{D}\left(t, \sigma_{t} ; S_{Y}^{\prime} S_{X} s_{3}, t_{0}, \tau\right)\right]
\end{aligned}
$$

where $s_{i}, f_{t i}$ and $t_{0}$ are the same for all modes.

\section{Simultaneous Signal PDF}

The explicit form of the signal lifetime PDFs based on the prototype PDF presented above are given below:

$$
\begin{aligned}
\mathcal{P}_{\pi \pi}^{D^{*+}}\left(t, \sigma_{t} ; \tau^{+}, \bar{\tau}^{+}\right) & =\left(1-f_{\mathrm{tag}}^{+}\right) \mathcal{R}\left(t, \sigma_{t} ; S_{\pi \pi} S_{\mathrm{tag}}^{\prime} s_{i}, t_{0}, \tau^{+}\right)+f_{\mathrm{tag}}^{+} \mathcal{R}\left(t, \sigma_{t} ; S_{\pi \pi} S_{\mathrm{tag}}^{\prime} s_{i}, t_{0}, \bar{\tau}^{+}\right), \\
\mathcal{P}_{\pi \pi}^{D^{*-}}\left(t, \sigma_{t} ; \tau^{+}, \bar{\tau}^{+}\right) & =\left(1-f_{\mathrm{tag}}^{-}\right) \mathcal{R}\left(t, \sigma_{t} ; S_{\pi \pi} S_{\mathrm{tag}}^{\prime} s_{i}, t_{0}, \bar{\tau}^{+}\right)+f_{\mathrm{tag}}^{-} \mathcal{R}\left(t, \sigma_{t} ; S_{\pi \pi} S_{\mathrm{tag}}^{\prime} s_{i}, t_{0}, \tau^{+}\right), \\
\mathcal{P}_{K K}^{D^{*+}}\left(t, \sigma_{t} ; \tau^{+}, \bar{\tau}^{+}\right) & =\left(1-f_{\mathrm{tag}}^{+}\right) \mathcal{R}\left(t, \sigma_{t} ; S_{K K} S_{\mathrm{tag}}^{\prime} s_{i}, t_{0}, \tau^{+}\right)+f_{\mathrm{tag}}^{+} \mathcal{R}\left(t, \sigma_{t} ; S_{K K} S_{\mathrm{tag}}^{\prime} s_{i}, t_{0}, \bar{\tau}^{+}\right), \\
\mathcal{P}_{K K}^{D^{*-}}\left(t, \sigma_{t} ; \tau^{+}, \bar{\tau}^{+}\right) & =\left(1-f_{\mathrm{tag}}^{-}\right) \mathcal{R}\left(t, \sigma_{t} ; S_{K K} S_{\mathrm{tag}}^{\prime} s_{i}, t_{0}, \bar{\tau}^{+}\right)+f_{\mathrm{tag}}^{-} \mathcal{R}\left(t, \sigma_{t} ; S_{K K} S_{\mathrm{tag}}^{\prime} s_{i}, t_{0}, \tau^{+}\right), \\
\mathcal{P}_{K \pi}^{D^{*} \pm}\left(t, \sigma_{t} ; \tau_{K \pi}\right) & =\mathcal{R}\left(t, \sigma_{t} ; S_{K \pi} S_{\mathrm{tag}}^{\prime} s_{i}, t_{0}, \tau_{K \pi}\right), \\
\mathcal{P}_{K K}^{\mathrm{unt}}\left(t, \sigma_{t} ; \tau^{+}, \bar{\tau}^{+}\right) & =\left(1-f_{D^{0}}\right) \mathcal{R}\left(t, \sigma_{t} ; S_{K K} S_{\mathrm{unt}}^{\prime} s_{i}, t_{0}, \bar{\tau}^{+}\right)+f_{D^{0}} \mathcal{R}\left(t, \sigma_{t} ; S_{K K} S_{\mathrm{unt}}^{\prime} s_{i}, t_{0}, \tau^{+}\right), \\
\mathcal{P}_{K \pi}^{\mathrm{unt}}\left(t, \sigma_{t} ; \tau_{K \pi}\right) & =\mathcal{R}\left(t, \sigma_{t} ; S_{K \pi} S_{\mathrm{unt}}^{\prime} s_{i}, t_{0}, \tau_{K \pi}\right),
\end{aligned}
$$

where $f_{\operatorname{tag}}^{ \pm}=0.2 \%, f_{D^{0}}=0.5$ and $S_{K \pi}=S_{\text {unt }}^{\prime}=1$ are fixed in the nominal fit. The offset $t_{0}$, the fractions $f_{t 1}, f_{t 2}$, the scaling factors $s_{1}, s_{2}, s_{3}, S_{\pi^{+} \pi^{-}}, S_{K^{+} K^{-}}, S_{\mathrm{tag}}^{\prime}$, along with the lifetime parameters $\tau^{+}, \bar{\tau}^{+}, \tau_{K \pi}$ are floating in the fit.

\subsubsection{Simultaneous Fit to the Signal-Only MC Datasets}

In order to test the signal PDF described above, we perform the seven-modes simultaneous fit to the 9 independent signal-only MC datasets (see Sec. 4.1). The proper time projections

\footnotetext{
${ }^{3} X$ indicates the final state and $Y$ the $D^{*}$ tag: $X=\{K \pi, K K, \pi \pi\}, Y=\{$ tag, unt $\}$.
} 
for the fit to one of these MC datasets, oneSignal1, are reported in Fig. 5.6. The signal PDF correctly describes the simulated events, as demonstrated by the Poisson pulls [53] reported below each projection.
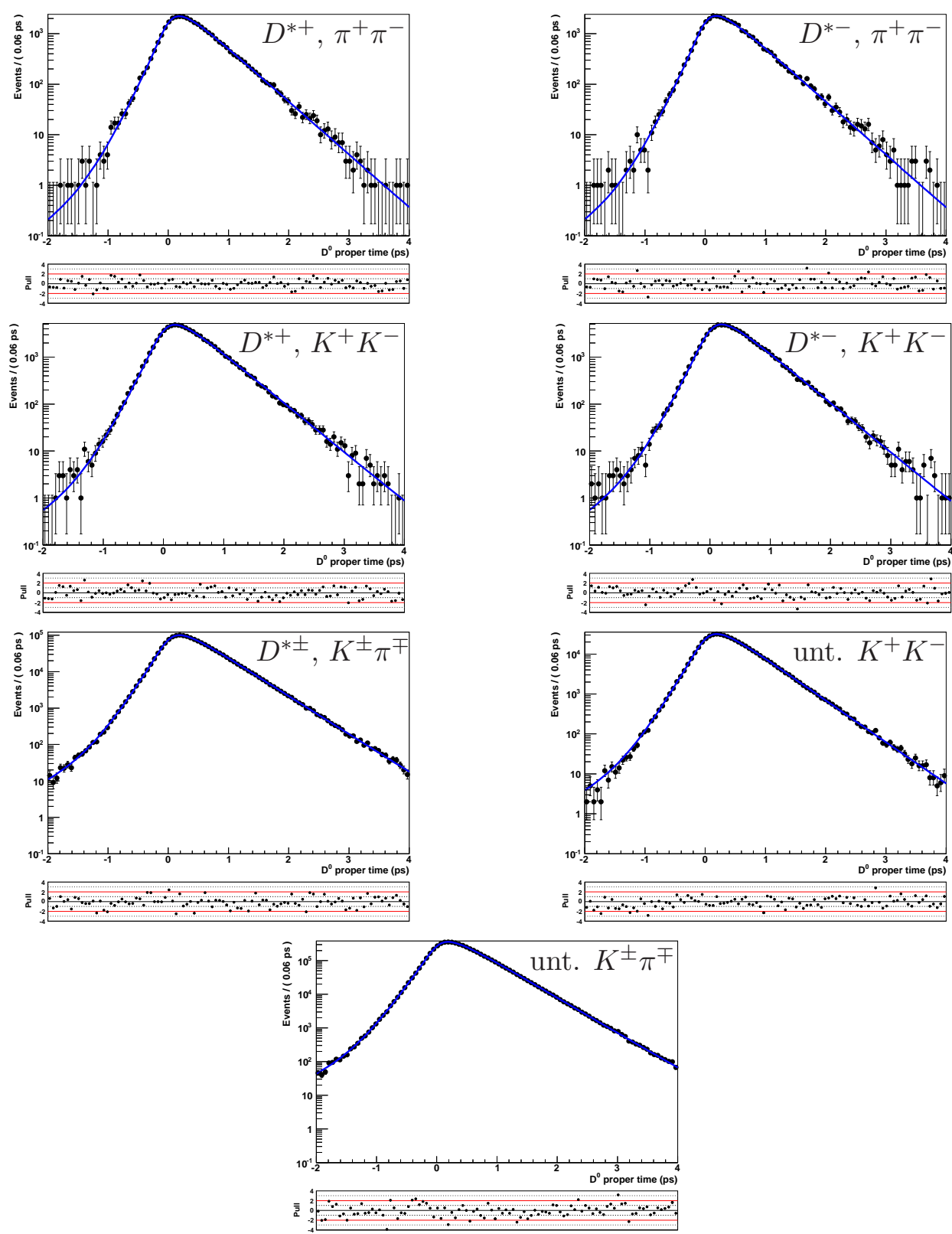

Figure 5.6: Proper time projections of the simultaneous fit to signal-only MC dataset oneSignal1.

The summary of the extracted values of $y_{C P}$ and $\Delta Y$ from the 9 independent $\mathrm{MC}$ datasets are reported in Fig. 5.7. In Tab. 5.5 we report the $y_{C P}$ and $\Delta Y$ weighted averages and their $\chi^{2}$ probability to be compatible with zero. The extracted values of $y_{C P}$ and $\Delta Y$ are compatible with no mixing and no $C P$ violation, as expected for simulated events. 

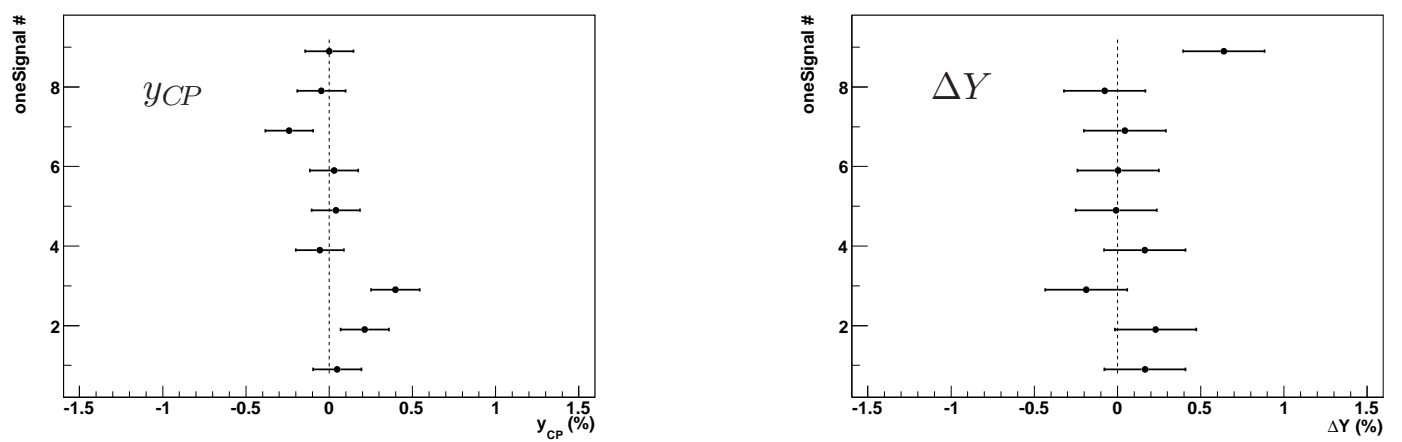

Figure 5.7: Summary of the extracted values of $y_{C P}$ (left) and $\Delta Y$ (right) in the simultaneous fit to the seven modes, signal-only events.

\begin{tabular}{c|cc}
\hline \hline observable & weighted average & $P\left(\chi^{2}\right)$ \\
\hline$y_{C P}$ & $0.005 \pm 0.048$ & $12 \%$ \\
$\Delta Y$ & $0.004 \pm 0.082$ & $33 \%$ \\
\hline \hline
\end{tabular}

Table 5.5: Weighted average and $\chi^{2}$ probability for being compatible with zero of the average values of $y_{C P}$ and $\Delta Y$ extracted from the simultaneous signal-only fit to the 9 independent MC datasets.

\subsection{Characterization of the Misreconstructed-Charm Events}

If the common ancestor of the $D^{0}$ decay products is a long-living charm meson, e.g. $D^{0}$, $D^{+}, \Lambda_{C}$, then the $D^{0}$ candidate belongs to the misreconstructed-charm background category. In the tagged channels, also the slow pion can be misidentified, but most of the misreconstructed-charm background is from real $D^{*+} \rightarrow D^{0} \pi^{+}$decays with a misreconstructed $D^{0}$ decay.

There are several sources contributing to this background, and they are highly non-uniform across the $m_{D^{0}}$ distribution and the in the $\left(m_{D^{0}}, \Delta m\right)$ plane, as can be seen for the tagged $K^{+} K^{-}$truth-matched events in the left plot of Fig. 5.8.
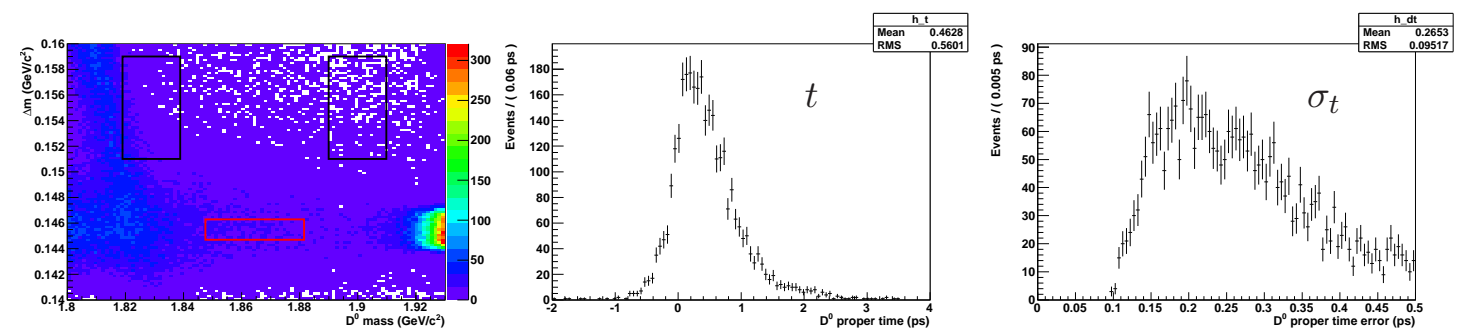

Figure 5.8: Misreconstructed-charm truth-matched distributions for the tagged $K^{+} K^{-}$ events: $\left(m_{D^{0}}, \Delta m\right)$ (left), proper time (center), and proper time error (right). The red lines indicate the signal region, the black lines indicate the sideband regions. The proper time and the proper time error distributions include only events in the signal region. 
In Appendix E.2 we report the relevant distributions for all the channels. In Tab. 5.6 we list the main components of this background in the signal region as estimated from the generic $c \bar{c}$ MC sample, bigCharm. The misreconstructed-charm is a long-lived background: the mean of all the proper time distributions (shown in Appendix E.2) is higher than the generated $D^{0}$ lifetime in MC, 411.67 fs.

\begin{tabular}{l|ccccc}
\hline \hline \multirow{2}{*}{ channel } & \multicolumn{3}{|c}{ Tagged } & \multicolumn{3}{c}{ Untagged } \\
& $\pi^{+} \pi^{-}$ & $K^{+} K^{-}$ & $K^{ \pm} \pi^{\mp}$ & $K^{+} K^{-}$ & $K^{ \pm} \pi^{\mp}$ \\
\hline$D^{0} \rightarrow \nu \ell X$ & $15.4 \%$ & $10.3 \%$ & $29.9 \%$ & $7.2 \%$ & $\leq 2 \%$ \\
$D^{0} \rightarrow K^{ \pm} \pi^{\mp}$ & $80.8 \%$ & $14.9 \%$ & $57.1 \%$ & $8.8 \%$ & $35.8 \%$ \\
$D^{0} \rightarrow \pi^{0} \pi^{+} K^{-}$ & $1.1 \%$ & $70.3 \%$ & $1.7 \%$ & $63.3 \%$ & $6.9 \%$ \\
$D^{+} \rightarrow \pi^{+} \pi^{+} K^{-}$ & $\leq 1 \%$ & $2.9 \%$ & $\leq 1 \%$ & $11.8 \%$ & $\leq 2 \%$ \\
$D^{0} \rightarrow K^{+} K^{-}$ & $\leq 1 \%$ & $\leq 1 \%$ & $1.3 \%$ & $\leq 1 \%$ & $3.5 \%$ \\
$D^{0} \rightarrow \pi^{+} \pi^{-}$ & $1.8 \%$ & $\leq 1 \%$ & $2.2 \%$ & $\leq 1 \%$ & $3.1 \%$ \\
$D^{0} \rightarrow \pi^{+} \pi^{-} \pi^{0}$ & $\leq 1 \%$ & $\leq 1 \%$ & $7.0 \%$ & $\leq 1 \%$ & $17.3 \%$ \\
$\Lambda$ decays & $\leq 1 \%$ & $\leq 1 \%$ & $\leq 1 \%$ & $4.9 \%$ & $2.6 \%$ \\
\hline total & 840 & 2720 & 5537 & 45280 & 40365 \\
\hline \hline
\end{tabular}

Table 5.6: Breakdown of the most important misreconstructed-charm background decay channels rates evaluated from the bigCharm MC dataset, and therefore not scaled to data luminosity. The $D^{0} \rightarrow K^{ \pm} \pi^{\mp}$ background channel in the tagged and untagged $K^{ \pm} \pi^{\mp}$ modes is due to doubly misidentified $K \pi$ decays.

In the tagged channels we do not observe any difference between the $D^{0}$ and the $\bar{D}^{0}$ misreconstructed-charm backgrounds. Therefore we do use the same shape to describe the events of this background category for the $D^{0} \rightarrow h^{+} h^{-}$and $\bar{D}^{0} \rightarrow h^{+} h^{-}$, with $h=K, \pi$.

In Tab. 5.7 we report the expected number of misreconstructed-charm events in the signal region and in the sidebands as estimated on the bigCharm MC dataset, rescaled to the data integrated luminosity.

\begin{tabular}{c|ccccc}
\hline \hline \multirow{2}{*}{ region } & \multicolumn{3}{|c}{ Tagged } & \multicolumn{2}{c}{ Untagged } \\
& $\pi^{+} \pi^{-}$ & $K^{+} K^{-}$ & $K^{ \pm} \pi^{\mp}$ & $K^{+} K^{-}$ & $K^{ \pm} \pi^{\mp}$ \\
\hline low sideband & 39 & 515 & 144 & 12347 & 10280 \\
signal region & 97 & 309 & 642 & 5477 & 4645 \\
high sideband & 5 & 97 & 19 & 4195 & 2206 \\
\hline \hline
\end{tabular}

Table 5.7: Expected number of misreconstructed-charm events in the signal region and the sidebands estimated from the bigCharm MC dataset and scaled to data integrated luminosity.

The events belonging to this category represent only the $\sim 0.1 \%$ of the events in the signal region ( $0.8 \%$ in the untagged $K^{+} K^{-}$channel) but because of their signal-like long lifetime we describe them separately from the rest of the background. 


\subsubsection{Parameterization}

The charm background is a signal-like long-lived background. We therefore describe it in a similar way as signal, using convolutions of an exponential with a Gaussian with per-event errors, $\mathcal{D}\left(t, \sigma_{t} ; s, t_{0}, \tau\right)$. The resolution is not expected to be the same as for signal events, since the $D^{0}$ is incorrectly reconstructed. In principle we should use a different lifetime and resolution for each of the different decay channels. However, given the low statistics of this background we have decided to use a sum of two convolutions, with different lifetimes, offsets and per-event error scaling factors. The misreconstructed-charm background PDF is therefore given by:

$$
\mathcal{P}_{X}^{\mathrm{crm}}\left(t, \sigma_{t}\right)=f_{\mathrm{crm}} \mathcal{D}\left(t, \sigma_{t} ; s_{1 \mathrm{crm}}, t_{0}^{1 \mathrm{crm}}, \tau_{1 \mathrm{crm}}\right)+\left(1-f_{\mathrm{crm}}\right) \mathcal{D}\left(t, \sigma_{t} ; s_{2 \mathrm{crm}}, t_{0}^{2 \mathrm{crm}}, \tau_{2 \mathrm{crm}}\right) .
$$

The proper time projections of the unbinned maximum likelihood fits to the misreconstructedcharm events in the signal region of the bigCharm MC dataset are reported in Fig. 5.9.

In Tab. 5.8 we report the values of the extracted lifetimes and fractions of the misreconstructedcharm PDF evaluated from the fit, for each of the five channels.

\begin{tabular}{l|ccccc}
\hline \hline \multirow{2}{*}{ parameter } & \multicolumn{3}{|c}{ Tagged } & \multicolumn{2}{c}{ Untagged } \\
& $\pi^{+} \pi^{-}$ & $K^{+} K^{-}$ & $K^{ \pm} \pi^{\mp}$ & $K^{+} K^{-}$ & $K^{ \pm} \pi^{\mp}$ \\
\hline$f_{\text {crm }}(\%)$ & 1 fixed & 1 fixed & $2.9 \pm 1.6$ & $7.9 \pm 1.1$ & $8.2 \pm 1.1$ \\
$\tau_{1 \text { crm }}(\mathrm{ps})$ & $0.46 \pm 0.03$ & $0.44 \pm 0.01$ & $0.82 \pm 0.35$ & $1.4 \pm 0.1$ & $0.99 \pm 0.07$ \\
$\tau_{2 \text { crm }}(\mathrm{ps})$ & - & - & $0.42 \pm 0.01$ & $0.476 \pm 0.008$ & $0.428 \pm 0.006$ \\
\hline \hline
\end{tabular}

Table 5.8: Extracted lifetimes of the misreconstructed-charm backgrounds in the signal region.

The PDFs extracted in these fits are used to characterize the misreconstructed-charm events in the signal region in the final fit. 

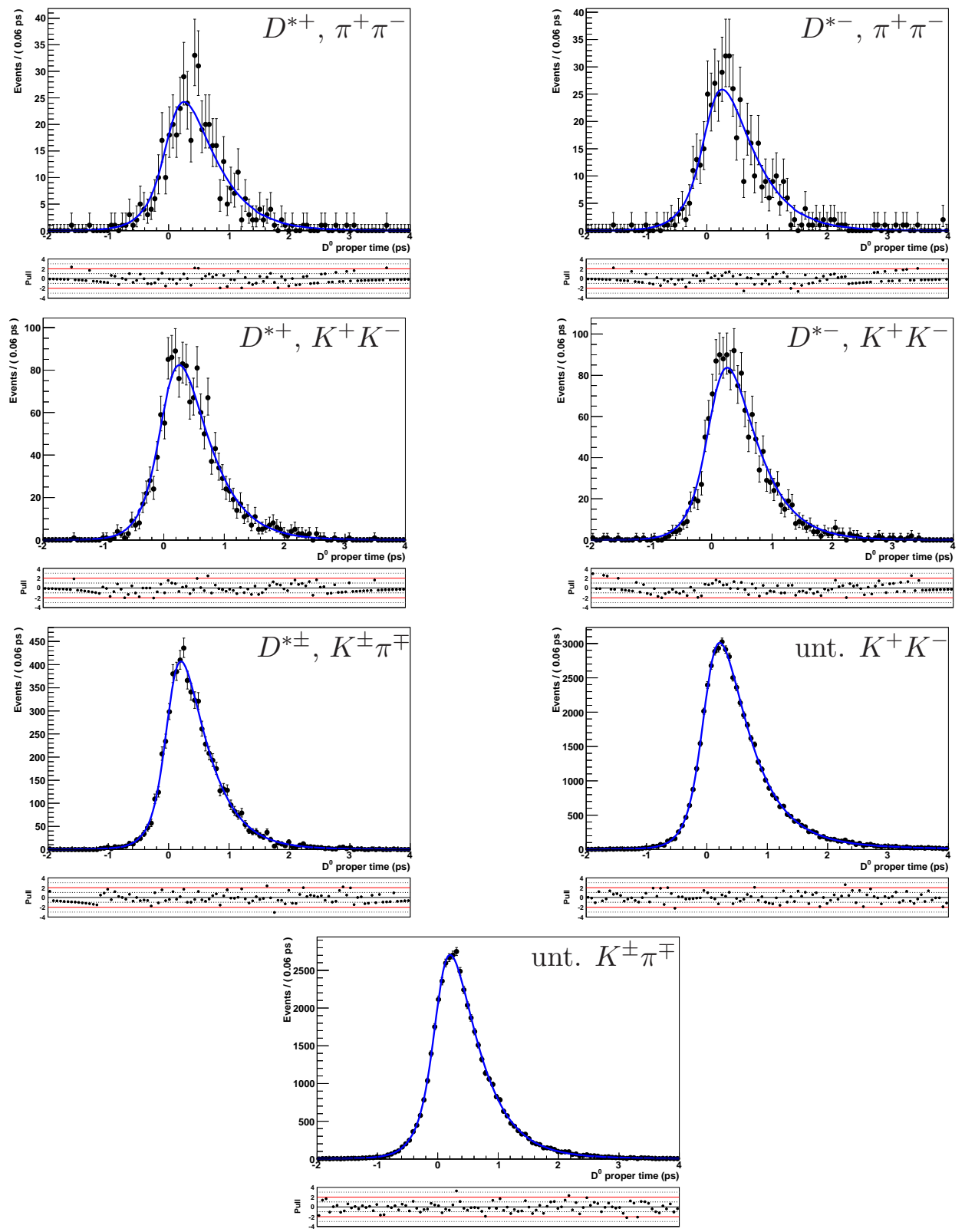

Figure 5.9: Proper time projections of the fits to the misreconstructed-charm events in the signal region of bigCharm MC dataset. For the tagged $\pi^{+} \pi^{-}$and $K^{+} K^{-}$channels one convolution was sufficient to describe the distribution. 


\subsection{Characterization of the Combinatorial Events}

The combinatorial-background category contains the events that are not classified as signal nor as misreconstructed-charm. Most of the combinatorial background are random combinations of tracks, with no effective lifetime.

In Fig. 5.10 we report the two dimensional distribution $\left(m_{D^{0}}, \Delta m\right)$ for the tagged $K^{+} K^{-}$ events, along with the proper time and proper time error distributions of the events in the signal region for the same channel.
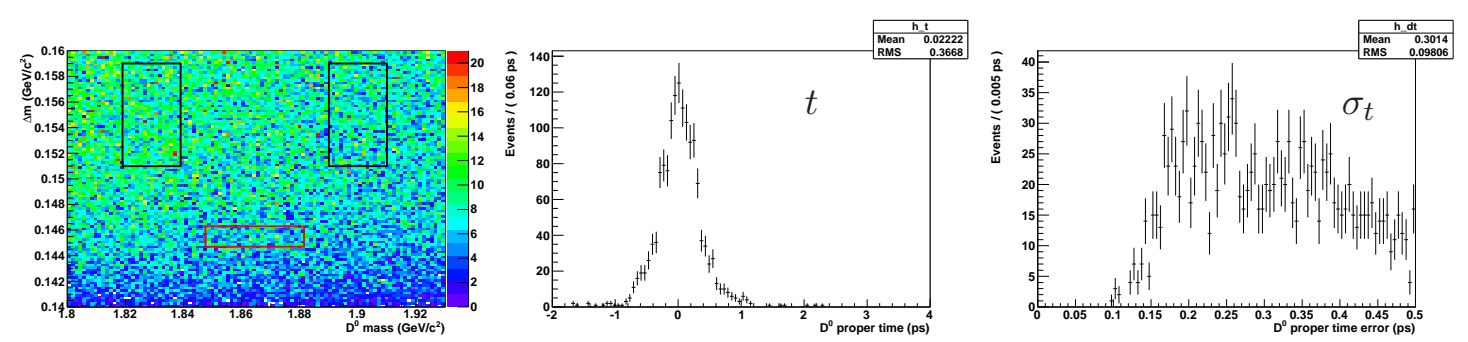

Figure 5.10: Combinatorial truth-matched distributions for the tagged $K^{+} K^{-}$events: $\left(m_{D^{0}}, \Delta m\right)$ (left), proper time (center), and proper time error (right). The red lines indicate the signal region, the black lines indicate the sideband regions. The proper time and the proper time error distributions include only events in the signal region.

In Appendix E.3 we report the relevant distributions from the simulated events for all the channels, as for the other two categories.

As for the misreconstructed-charm category, in the tagged channels, there is no significant difference between the $D^{0}$ and the $\bar{D}^{0}$ combinatorial backgrounds. Therefore we do use the same shape to describe the events of this background category for the $D^{0} \rightarrow h^{+} h^{-}$ and $\bar{D}^{0} \rightarrow h^{+} h^{-}$, with $h=K, \pi$.

The two-dimensional $\left(t, \sigma_{t}\right)$ combinatorial PDF is obtained from the data mass sidebands. In Fig. 5.11 and 5.12 we report the comparison between the combinatorial events in the sidebands and the ones in the signal region for the proper time and the proper time error distributions. There are small discrepancies between the sideband distributions and the signal region ones. We build the combinatorial PDF as a weighted average of the combinatorial PDFs extracted in the two sidebands, as described in the following section.

\subsubsection{Parameterization}

In all five decay channels, the main background is composed of combinatorial candidates. Hence we want to obtain a PDF that appropriately describes the combinatorial background in the signal region that, possibly, does not rely on simulated events.

We obtain the combinatorial PDF for each of the five channels from the sidebands by forming a weighted average of the PDFs extracted from the low $\left(\mathcal{P}_{X}^{1 S B}\right)$ and the high 

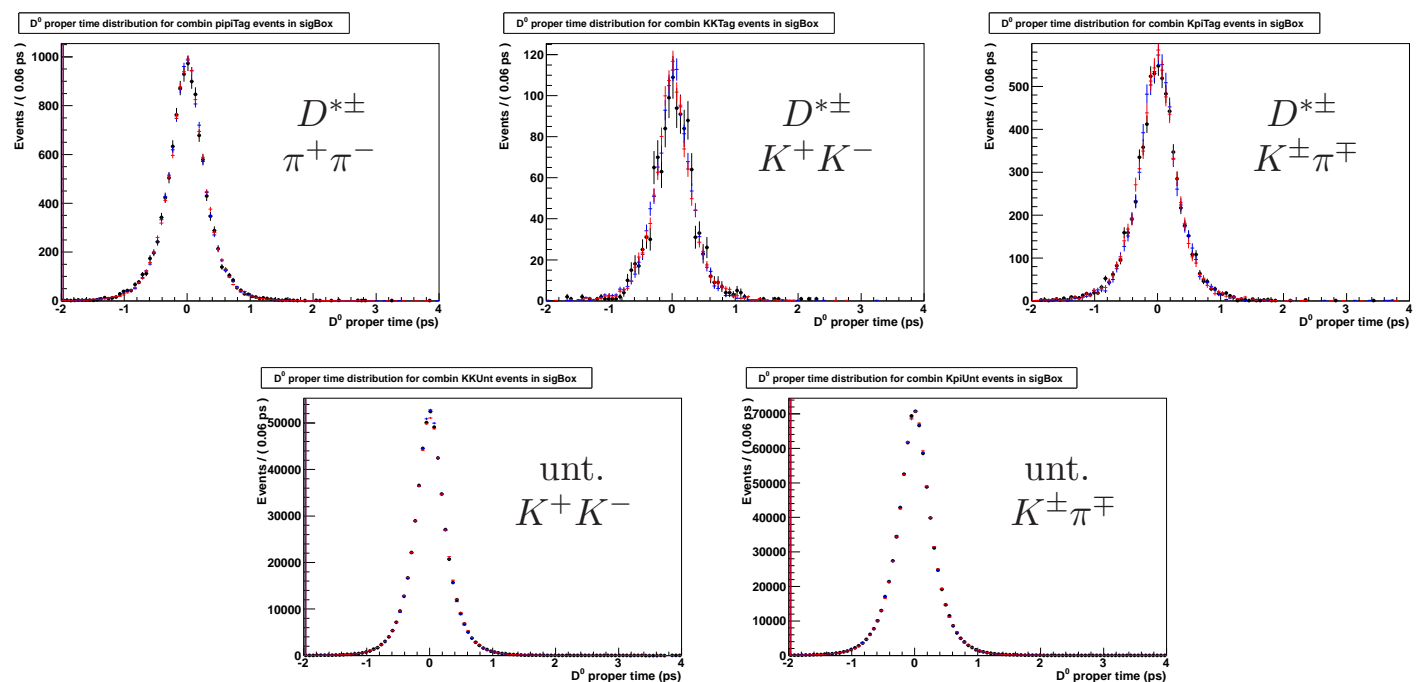

Figure 5.11: Comparison of the proper time distribution in the signal region (black), low (red) and high (blue) sidebands for the combinatorial events of the bigCombin MC dataset.
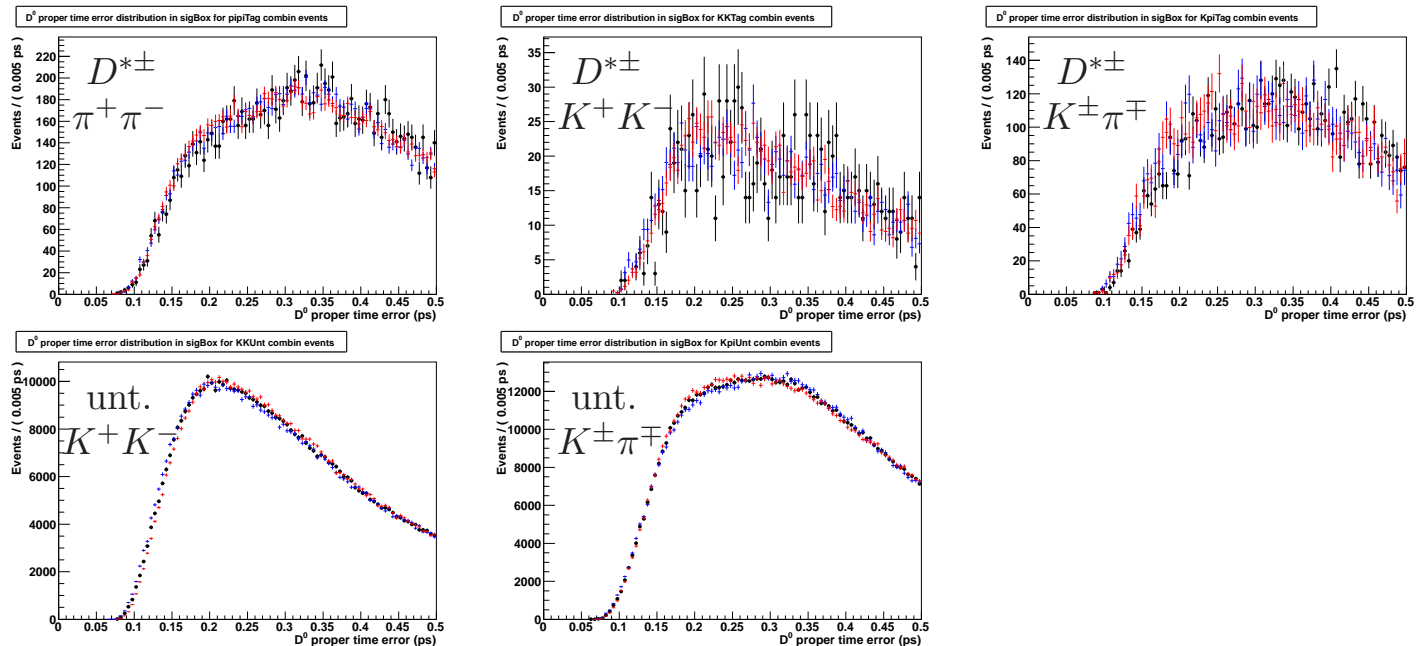

Figure 5.12: Comparison of the proper time error distribution in the signal region (black), low (red) and high (blue) sidebands for the combinatorial events of the bigCombin MC dataset. 
$\left(\mathcal{P}_{X}^{\mathrm{hSB}}\right)$ sideband:

$$
\mathcal{P}_{X}^{\mathrm{comb}}=h \mathcal{P}_{X}^{\mathrm{lSB}}+(1-h) \mathcal{P}_{X}^{\mathrm{hSB}}
$$

The form of the sideband PDFs $\mathcal{P}_{X}^{1 \mathrm{ISB}}$ and $\mathcal{P}_{X}^{\mathrm{hSB}}$ depends on the decay channel and is given in Tab. 5.9. Their extraction in each of the five channels is discussed below.

\begin{tabular}{c|l}
\hline \hline channels & form of the combinatorial PDFs $\mathcal{P}_{X}^{1 \mathrm{ISB}}$ and $\mathcal{P}_{X}^{\mathrm{hSB}}$ \\
\hline tagged & 2d histogram with fixed bin-width, 100 bins in $t$ and 100 in $\sigma_{t}$ \\
untagged $K^{+} K^{-}$ & sum of 3 convolutions of an exponential with a Gaussian (see Eq. 5.13) \\
untagged $K^{ \pm} \pi^{\mp}$ & 2d histogram with adaptive binning (see Fig. 5.14) \\
\hline \hline
\end{tabular}

Table 5.9: Forms of the combinatorial PDFs describing the events in the sidebands for the different channels. The PDFs are described in more detail in the following. " $2 \mathrm{~d}$ " stands for two-dimensional.

The weighting parameter $h$ is extracted from the bigCombin MC dataset and assumed to correctly describe data. A systematic error on this assumption is discussed in Sec. 6.2.4. The values of the extracted weighting parameters that are used in the fit to data are all near 0.5 and are reported in Tab. 5.10.

\begin{tabular}{c|ccccc}
\hline \hline & \multicolumn{3}{|c}{ Tagged } & \multicolumn{2}{c}{ Untagged } \\
& $\pi^{+} \pi^{-}$ & $K^{+} K^{-}$ & $K^{ \pm} \pi^{\mp}$ & $K^{+} K^{-}$ & $K^{ \pm} \pi^{\mp}$ \\
\hline$h$ & $0.496 \pm 0.023$ & $0.518 \pm 0.030$ & $0.542 \pm 0.024$ & $0.497 \pm 0.017$ & $0.520 \pm 0.004$ \\
\hline \hline
\end{tabular}

Table 5.10: Values of the weighting parameters used to build the combinatorial PDF for the signal region events from the ones obtained in the sidebands, extracted from the bigCombin MC dataset.

In the following we describe the details of the construction of the combinatorial PDF $\mathcal{P}_{X}^{\text {comb }}$, for each of the five channels $X$ : the form and the extraction of the sideband PDFs $\mathcal{P}_{X}^{\mathrm{lSB}}$ and $\mathcal{P}_{X}^{\mathrm{hSB}}$, and the evaluation of the weighting parameter. The low (high) sideband region also contains some signal and misreconstructed-charm events, as shown in Tab. 5.11 (5.12). Therefore we first present the PDFs for these two categories for the sideband events.

\section{Signal and Misreconstructed-Charm PDFs in the Sidebands}

The signal and misreconstructed-charm events in the sidebands are estimated directly from simulated events. A systematic error will be assigned for this assumption.

In the untagged $K^{ \pm} \pi^{\mp}$ and the three tagged channels the combinatorial PDF in the sidebands is a $2 \mathrm{~d}$ histogram (see Tab 5.9), we therefore use a $2 \mathrm{~d}$ histogram with the same binning for the signal and misreconstructed-charm PDF in the sidebands. We use the bigSignal and bigCharm MC datasets to build these histogram, and rescale them to data luminosity. 


\begin{tabular}{c|ccccc}
\hline \hline low & \multicolumn{3}{|c}{ Tagged } & \multicolumn{2}{c}{ Untagged } \\
sideband & $\pi^{+} \pi^{-}$ & $K^{+} K^{-}$ & $K^{ \pm} \pi^{\mp}$ & $K^{+} K^{-}$ & $K^{ \pm} \pi^{\mp}$ \\
\hline signal & 40 & 23 & 555 & 2107 & 45697 \\
misrec. charm & 39 & 515 & 144 & 12347 & 10280 \\
combinatorial & 12626 & 1380 & 7328 & 147548 & 587985 \\
\hline \hline
\end{tabular}

Table 5.11: Estimation of the number of signal and misreconstructed-charm events in the low mass sideband. These numbers are estimated using the bigSignal and bigCharm MC datasets and rescaled to match data integrated luminosity. We also report the number of combinatorial events, estimated and rescaled from the bigCombin MC dataset.

\begin{tabular}{c|ccccc}
\hline \hline high & \multicolumn{3}{|c}{ Tagged } & \multicolumn{2}{c}{ Untagged } \\
sideband & $\pi^{+} \pi^{-}$ & $K^{+} K^{-}$ & $K^{ \pm} \pi^{\mp}$ & $K^{+} K^{-}$ & $K^{ \pm} \pi^{\mp}$ \\
\hline signal & 11 & 7 & 156 & 509 & 8362 \\
misrec. charm & 5 & 97 & 19 & 4195 & 2206 \\
combinatorial & 11726 & 1201 & 6729 & 123153 & 511580 \\
\hline \hline
\end{tabular}

Table 5.12: Estimation of the number of signal and misreconstructed-charm events in the high mass sideband. These numbers are estimated using the bigSignal and bigCharm $\mathrm{MC}$ datasets and rescaled to match data integrated luminosity. We also report the number of combinatorial events, estimated and rescaled from the bigCombin MC dataset.

In the untagged $K^{+} K^{-}$channel we proceed in a different way: since we use an analytic function to describe the combinatorial component, as described in the next section, we also use an analytic function for the signal and misreconstructed-charm events. The analytic functions are fitted to MC datasets and fixed in the fit to the sidebands. We use the same analytic function for the signal and misreconstructed-charm components, the sum of two convolutions of an exponential with a Gaussian:

$$
\mathcal{P}_{h h}^{\text {side }}\left(t, \sigma_{t}\right)=f_{\text {side }} \mathcal{D}\left(t, \sigma_{t} ; s_{1 \text { side }}, t_{0}^{\text {side }}, \tau_{\text {side }}\right)+\left(1-f_{\text {side }}\right) \mathcal{D}\left(t, \sigma_{t} ; s_{2 \text { side }}, t_{0}^{\text {side }}, \tau_{\text {side }}\right) .
$$

As usual we multiply the above PDF by the proper time error PDF, $\mathcal{H}\left(\sigma_{t}\right)$, so that $\mathcal{P}^{\text {side }}\left(t, \sigma_{t}\right) \cdot \mathcal{H}\left(\sigma_{t}\right)$ is a properly normalized two-dimensional PDF. The $\sigma_{t} \mathrm{PDF}$ is taken to be the one-dimensional histogram, built with simulated events.

For what concerns the signal component, we apply a correction to the extracted lifetimes due to the fact that the generated $D^{0}$ lifetime in $\mathrm{MC}$ is $411.67 \mathrm{fs}$ while in data we have measured shorter lifetimes. To estimate the correction we computed the weighted average of the $D \rightarrow K \pi$ and the $D^{0}, \bar{D}^{0} \rightarrow h^{+} h^{-}$lifetimes extracted in the previous analyses [50, 51], obtaining:

$$
\begin{aligned}
\left\langle\tau_{K \pi}\right\rangle & =(410.16 \pm 0.33) \mathrm{fs} \\
\left\langle\tau_{h h}\right\rangle & =(404.95 \pm 0.97) \mathrm{fs} .
\end{aligned}
$$

We apply a shift of $411.67-410.16=1.51 \mathrm{fs}$ to the extracted signal lifetimes of the tagged 
and untagged $K^{ \pm} \pi^{\mp}$ channels in the sidebands, and a shift of $411.67-404.95=6.72$ fs to the other channels. We will assign a systematic error varying the shift by the statistical error reported in the equations above. The correction is applied only to the signal lifetime in the sidebands.

\section{Combinatorial PDF for the Tagged Channels}

In both sidebands the combinatorial PDF consists of a two-dimensional histogram in $(t$, $\sigma_{t}$ ), with 100 bins for each variable. The sideband PDFs $\mathcal{P}_{X}^{1 \mathrm{SB}}$ and $\mathcal{P}_{X}^{\mathrm{hSB}}$ are extracted from the sideband histograms after subtracting the signal and the misreconstructed-charm components. The signal and misreconstructed-charm histograms are built using the bigSignal and bigCharm MC datasets. The subtraction operation can be tricky, especially in the low-statistics bins: it can happen that in one or more bins the result of the subtraction is negative, in this case we assign 0 to the content of the bin. If this happens in too many bins, then the subtracted histogram is not a good description of the combinatorial events in the sidebands: every time we assign 0 we are neglecting a signal (or misreconstructedcharm) contribution. Studies on the simulated events have demonstrated that this is not a problem for the tagged channels.

The weighting parameter $h$ is extracted from the simulated events. First we obtain the low and high sideband PDFs from the truth-matched combinatorial events of the bigCombin $\mathrm{MC}$ dataset. Then we fit their weighted average to the combinatorial events in the signal region and extract the value of $h$. In Fig. 5.13 we report the results of these fits that yields the values of the weighting parameters $h$ reported in Tab. 5.10.

Once we have obtained $\mathcal{P}_{X}^{\mathrm{ISB}}$ and $\mathcal{P}_{X}^{\mathrm{hSB}}$ from the sidebands and $h$ from the simulated events, we build the weighted average of the PDFs as in Eq. (5.9) and obtain the PDF for the combinatorial events in the signal region for the tagged channel $X, \mathcal{P}_{X}^{\text {comb }}$.

\section{Combinatorial PDF for the Untagged $K^{ \pm} \pi^{\mp}$ Channel}

In the untagged $K^{ \pm} \pi^{\mp}$ channel $\mathrm{MC}$ studies have shown that the subtraction procedure suffers from the problem we have mentioned above in the low-statistics bins. In order to overcome this problem we have changed the binning of the $2 \mathrm{~d}$ histogram: we use an adaptive binning maintaining the same bin width in the regions where the distribution changes rapidly and merging two or more bins together when the distribution is less steep. This procedure solves the subtraction issue for this untagged channel. In Fig. 5.14 we report a picture illustrating the adaptive binning.

The weighting parameter $h$ is extracted with the same procedure used for the tagged channels. In Fig. 5.15 we report the results of the fit that yield the value of the weighting parameters $h$ reported in Tab. 5.10. 

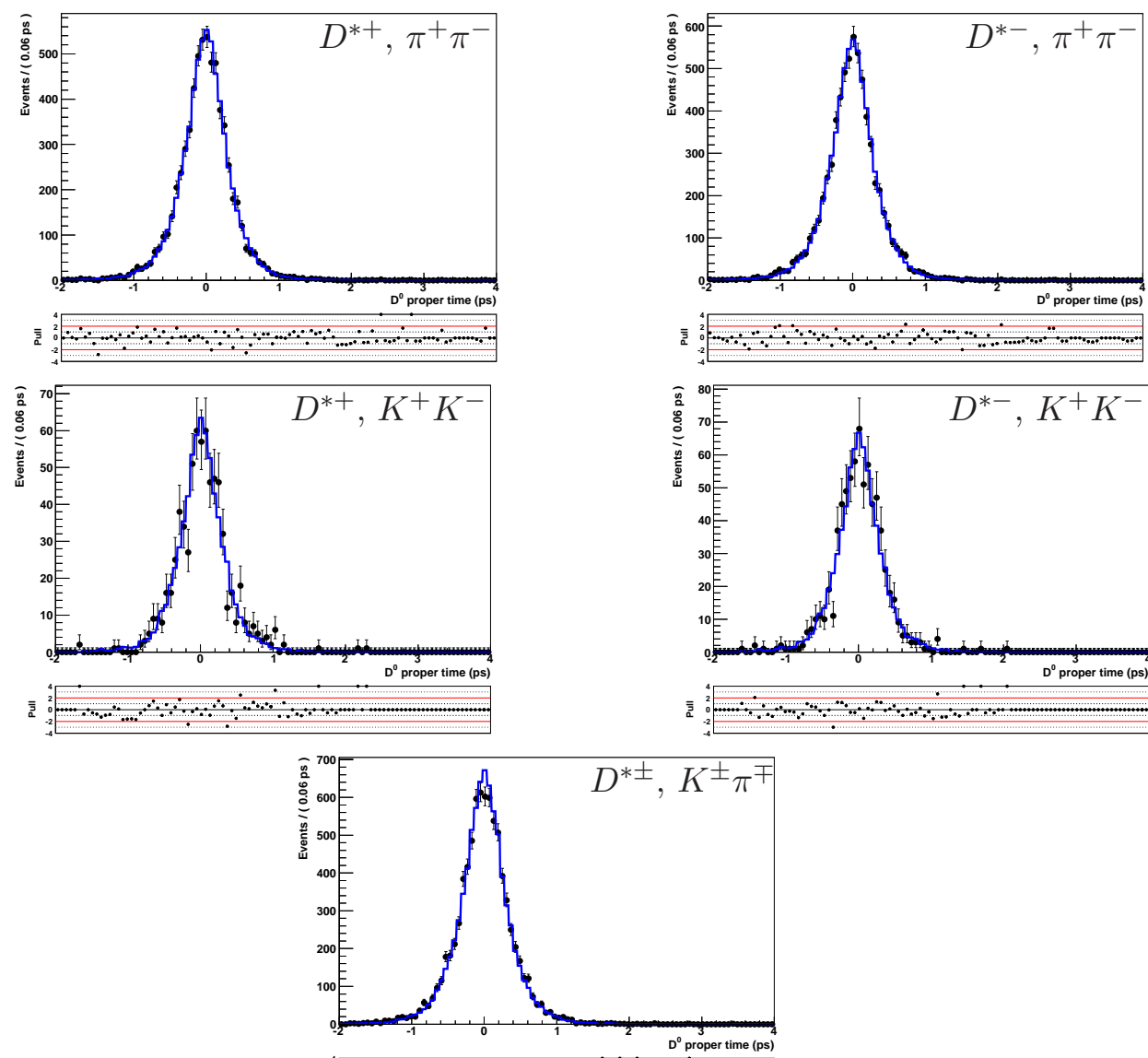

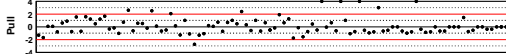

Figure 5.13: Results of the fits to the signal region events of bigCombin MC dataset of the weighted average of the low and high sideband PDF for tagged channels that allowed the determination of the weighting parameter $h$.

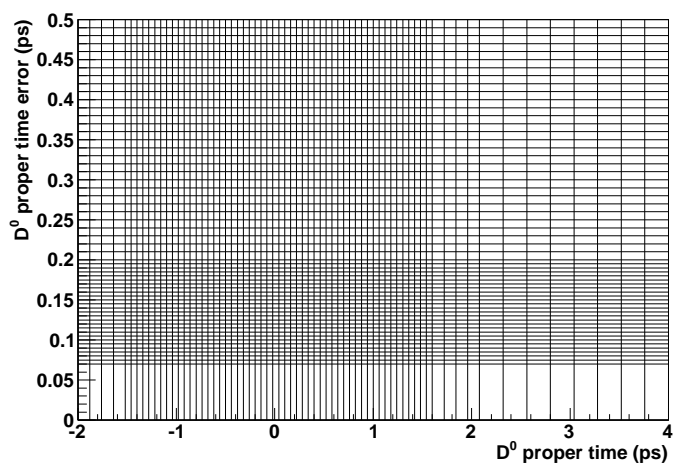

Figure 5.14: Picture of the adaptive binning adopted for the combinatorial PDF of the untagged $K^{ \pm} \pi^{\mp}$ channel for the validation fits to MC. The one adopted for the nominal fit to data is the same, except in the region from $-0.68 \mathrm{ps}$ to $0.7 \mathrm{ps}$, where the division is 10 times finer. 


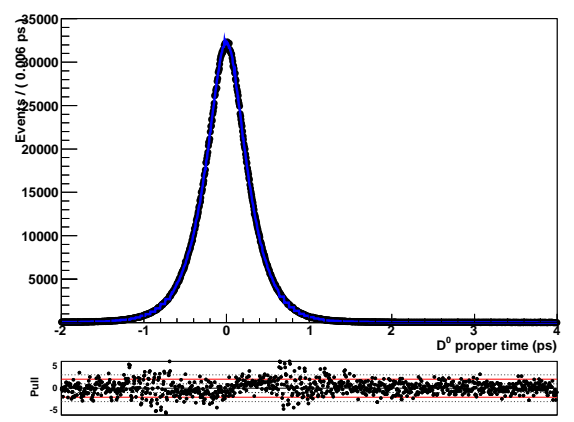

Figure 5.15: Result of the fit to the signal region events of bigCombin MC dataset of the weighted average of the low and high sideband PDF for the untagged $K^{ \pm} \pi^{\mp}$ channel. The binning of the Poisson pulls reported below the plot is chosen to match the binning of the histogram PDF.

\section{Combinatorial PDF for the Untagged $K^{+} K^{-}$Channel}

The untagged $K^{+} K^{-}$channel is the one with the highest fraction of combinatorial events in the signal region. MC studies have demonstrated that the subtraction issue was not completely solved using adaptive binning, even after trying several binnings. Moreover, choosing the binning that gave the best answer on the subtraction side and fitting the single channel, we observed that the offset of the signal resolution function was not compatible with zero, even if the lifetime was extracted correctly. Fitting the untagged channels together, since the offset is driven by the untagged $K^{ \pm} \pi^{\mp}$ channel, the untagged $K^{+} K^{-}$ lifetime was extracted $2 \mathrm{fs}$ below the input value. This study was performed on simulated events.

We therefore chose to use an analytic function in order to describe the combinatorial events in the untagged $K^{+} K^{-}$sidebands (and signal region). The function is basically the same as the signal PDF with the only difference that we allow for 3 different lifetimes and offsets, one for each component of the conditional PDF. The proper time PDF for untagged $K^{+} K^{-}$combinatorial background candidates is therefore given by:

$$
\begin{aligned}
\mathcal{P}_{\mathrm{unt} K K}^{\mathrm{comb}}\left(t, \sigma_{t}\right)= & f_{1 \mathrm{comb}} \mathcal{D}\left(t, \sigma_{t} ; s_{1 \mathrm{comb}}, t_{0}^{1 \mathrm{comb}}, \tau_{1 \mathrm{comb}}\right)+ \\
& \left(1-f_{1 \mathrm{comb}}\right)\left[f_{21 \mathrm{comb}} \mathcal{D}\left(t, \sigma_{t} ; s_{2 \mathrm{comb}}, t_{0}^{2 \mathrm{comb}}, \tau_{2 \mathrm{comb}}\right)+\right. \\
& \left.\left(1-f_{21 \mathrm{comb}}\right) \mathcal{D}\left(t, \sigma_{t} ; s_{3 \mathrm{comb}}, t_{0}^{3 \mathrm{comb}}, \tau_{3 \mathrm{comb}}\right)\right]
\end{aligned}
$$

where $\mathcal{D}\left(t, \sigma_{t} ; s, t_{0}, \tau\right)$ is defined in Eq. (5.1).

In Fig. 5.16 we report the fits to data sidebands that allow one to extract $\mathcal{P}_{X}^{1 \mathrm{SB}}$ and $\mathcal{P}_{X}^{\mathrm{hSB}}$. In these fits the signal and the misreconstructed-charm PDFs and their expected number of events are fixed.

The weighting parameter $h$ is extracted with the same procedure used for the other channels. In Fig. 5.17 we report the results of the fit that yields the value of the weighting 

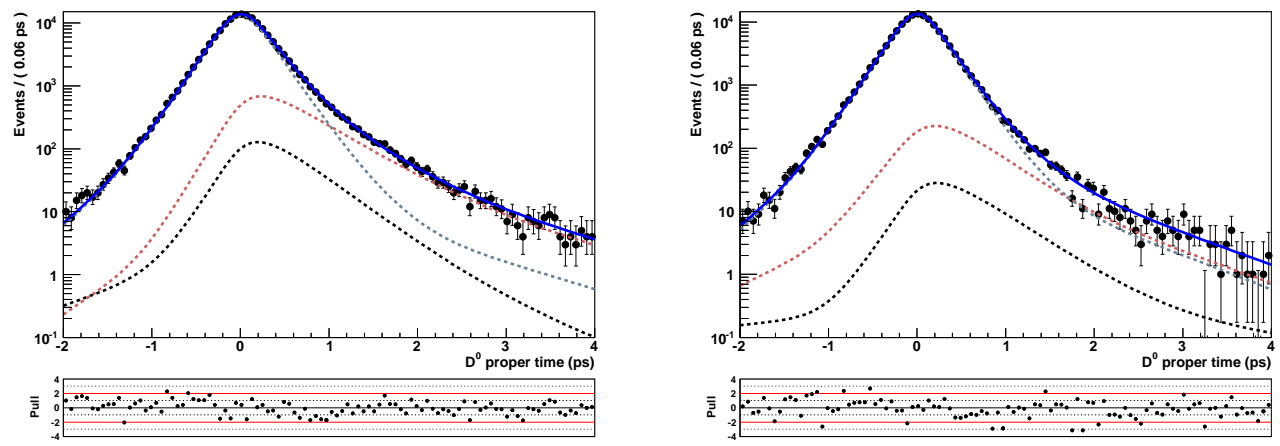

Figure 5.16: Results of the fits to the low (left) and high (right) data sidebands for the untagged $K^{+} K^{-}$channel. In black the signal and in red the misreconstructed-charm PDFs, both fixed in the fit; the dashed blue line represents the extracted combinatorial PDF.

parameters $h$ reported in Tab. 5.10.

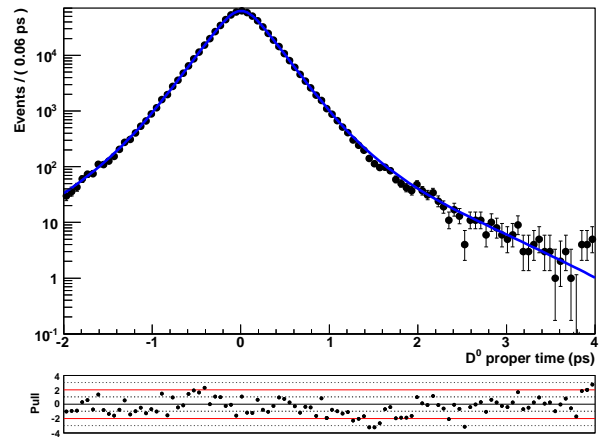

Figure 5.17: Results of the fits to the signal region events of bigCombin MC dataset of the weighted average of the low and high sideband PDF for the untagged $K^{+} K^{-}$channel.

\subsection{Proper Time Error PDF}

The $\sigma_{t} \mathrm{PDF}$ multiplies the proper time PDF since the latter also depends on the proper time error. In this analysis they are described by one-dimensional histograms, $\mathcal{H}\left(\sigma_{t}\right)$. The proper time error distributions are different for signal and background candidates across the different fitting regions. It is important to obtain a good description of these distributions for the different background and signal components in each of the fitting regions in order to avoid fitting biases.

The proper time error distribution for the signal component in the signal region is obtained by subtracting the combinatorial and the misreconstructed-charm background $\sigma_{t}$ distribution to the $\sigma_{t}$ distribution of all the $D^{0}$ candidates in the signal region. Therefore in the following we describe how we build the $\mathcal{H}\left(\sigma_{t}\right)$ for each of the three categories in each of the five channels. 


\section{Backgrounds Proper Time Error Distributions}

The proper time error PDF for misreconstructed-charm events is obtained directly from the simulated events, exploiting the full statistics of the bigCharm MC dataset. We build the one-dimensional $\sigma_{t}$ distribution and normalize it to unity and obtain $\mathcal{H}\left(\sigma_{t}\right)$ for the five channels.

The $\sigma_{t} \mathrm{PDF}$ for the combinatorial background is obtained from the mass sidebands with the same procedure exploited for the two-dimensional $\left(t, \sigma_{t}\right)$ PDF. First we obtain the combinatorial proper time error distribution in the sidebands subtracting the signal and misreconstructed-charm contributions (both evaluated on the simulated events) to the total $\sigma_{t}$ distribution. Then we combine the proper time error distributions obtained in the sidebands with the weighting parameter used to weight the two-dimensional PDF.

\section{Signal Proper Time Error Distributions}

As mentioned above, the signal $\sigma_{t} \mathrm{PDF}$ is build by subtraction once the background $\sigma_{t}$ PDFs and expected number of events in the signal region are know. As can be seen from Fig. 5.18 the agreement of the signal truth-matched distribution and the subtracted one is very good for simulated events, proving the fact that the combinatorial $\sigma_{t}$ distributions of the events in the mass sidebands describe well the combinatorial events in the signal region.
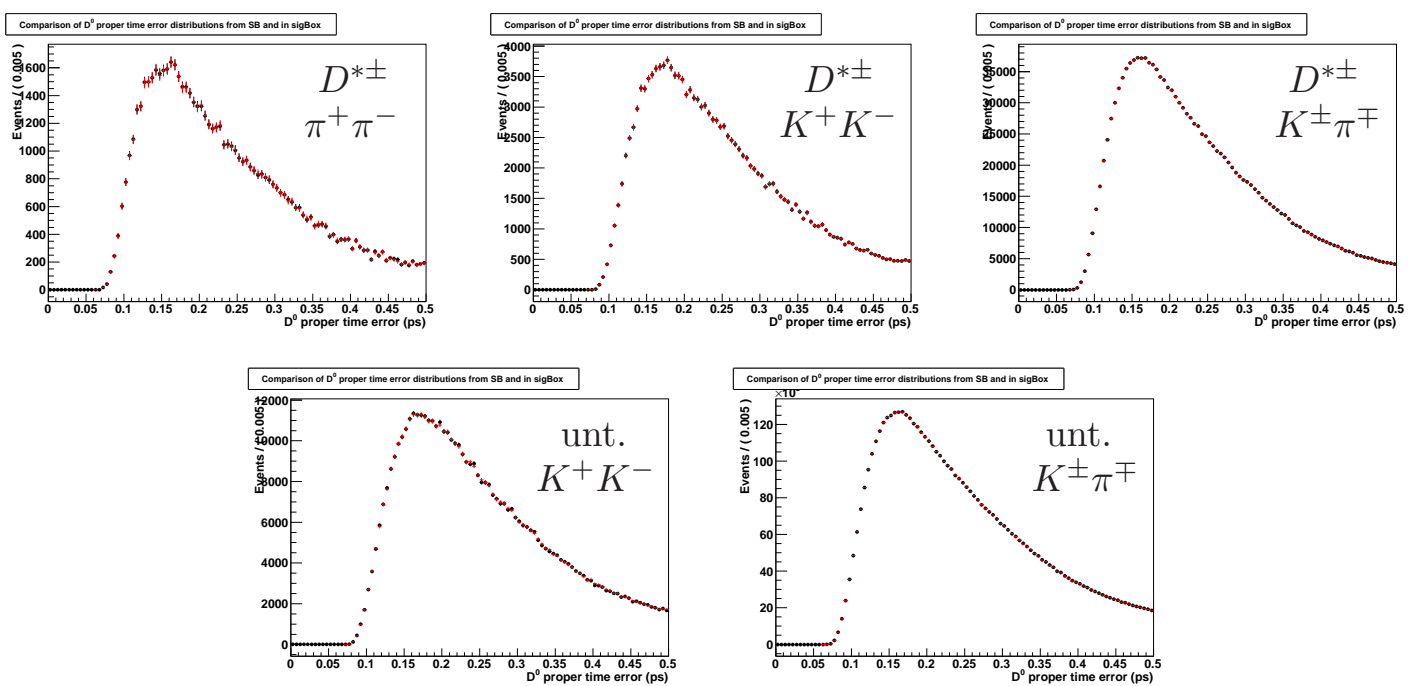

Figure 5.18: Comparison of the proper time error distribution of the truth matched signal events in the signal region (black) VS the proper time error distribution obtained from the subtraction of the misreconstructed-charm and combinatorial $\sigma_{t}$ distributions from the distribution of the events in the signal region of cocktail1 (red). The KolmogorovSmirnov tests for these distributions are all above $99.9 \%$.

This operation requires the knowledge of the expected number of background events in the signal region. As described in Sec. 4.4, the expected number of misreconstructed-charm 
events is evaluated from the simulated events, while the combinatorial yields are evaluated from the mass fits, except for the untagged $K^{+} K^{-}$channel. In this channel, the expected number of combinatorial events is a floated parameter in the fit, and it is therefore unknown prior to the fit. To overcome this problem we proceed in the following way: we perform the simultaneous fit a first time using the expected number of combinatorial untagged $K^{+} K^{-}$events as estimated from the mass fit. We then re-do the fit a second time using the number of combinatorial untagged $K^{+} K^{-}$events just extracted. We consider this one as the nominal fit. In principle we should iterate this operation until we converge on a stable solution. Since this would require a certain unknown number of iterations we have decided to perform it only once and assign a systematic error. In the validation fits to simulated events we have used the information of the MC truth.

\subsection{Simultaneous Fit}

The effective lifetimes $\tau^{+}$and $\bar{\tau}^{+}$and the $D^{0}$ lifetime $\tau_{K \pi}$ are determined with an unbinned extended maximum likelihood fit to the reconstructed proper time and proper time error of the candidates in the signal region. As in the previous $y_{C P}$ analyses, we only fit candidates with proper times $t$ and proper time errors $\sigma_{t}$ satisfying $-2 \mathrm{ps}<t<4$ ps and $\sigma_{t}<0.5 \mathrm{ps}$.

Before attempting to fit the simulated events, and then data, we check that generating a sample from the total PDF and fitting it, we obtain values of the parameters that are statistically compatible with the generated ones. We build the total PDF with reasonable values of the parameters as estimated from the studies presented up to now. We set the three lifetimes to the same value, therefore we expect no mixing and no $C P V$. We generate 100 samples using the total PDF and fit them. In 8 fits the covariance matrix was not positive-definite and those fits are excluded from the studies. In Fig. 5.19 we report the histograms of the pulls, defined as "(fitted value - generated value) / error", of the three lifetimes $\tau^{+}, \bar{\tau}^{+}$and $\tau_{K \pi}$. Using the covariance matrix returned from the fit we have also computed the values of $y_{C P}$ and $\Delta Y$, and we report the corresponding pulls for the observables. We fit the pulls distribution with a Gaussian, the fit results are reported on the plots.

The mean values of all the parameters are compatible with zero, this suggests that, given certain values of the PDF parameters, we are able to obtain them back from the fit. The width of the Gaussians are compatible with one, therefore the error from the fit is a good estimator of the statistical error.

In the following we briefly review the fit strategy and then we present the results of the fit to MC cocktails (in Sec. 5.5.2) and to data (in Sec. 5.5.3). 

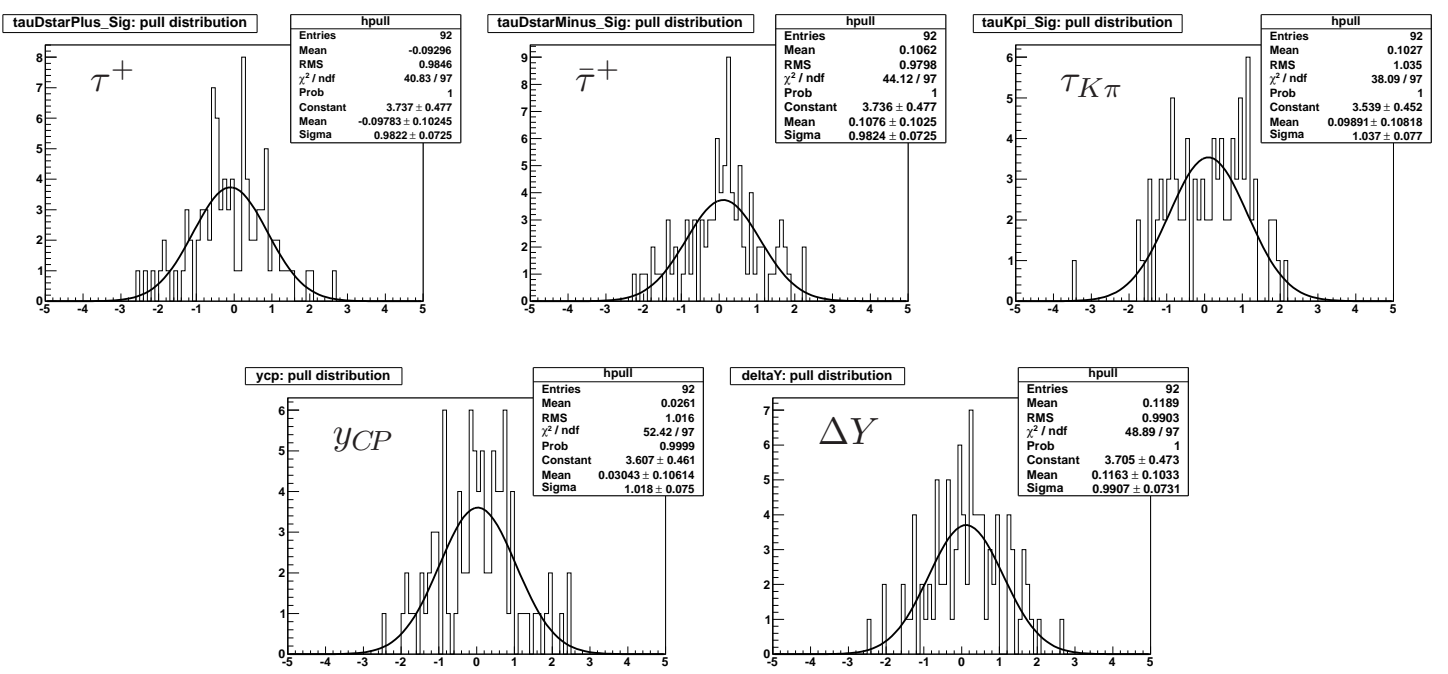

Figure 5.19: Histograms of the pulls of the three lifetimes and of $y_{C P}$ and $\Delta Y$, extracted from the 100 Toys MC. Results of the fit with a single Gaussian are reported on each plot.

\subsubsection{Fit Strategy}

The fit to the proper time and proper time error is performed in two steps. First we extract the shapes and the expected number of events of the background categories. The background shapes and yields are fixed for all the channels, except for the untagged $K^{+} K^{-}$ combinatorial yields, and a first fit to data is performed. As mentioned in Sec. 5.4 the estimation of the expected number of combinatorial events in the untagged $K^{+} K^{-}$channel from the mass fit is not accurate enough, therefore we extract a better estimation from the first fit and we repeat the fit to data a second time. The second fit is considered the nominal fit.

In the following we summarize the procedure to obtain the shape and yields of the events in the background categories.

\section{Misreconstructed-Charm Shape and Yields}

To evaluate the misreconstructed-charm shape and yields we use the bigCharm MC dataset. For each of the 5 channels we fit the truth-matched misreconstructed-charm events in the signal region using the PDF described in Sec. 5.2.1. The expected number of misreconstructedcharm events in the signal region is evaluated re-scaling the number of the truth-matched events of the MC dataset to data integrated luminosity. 


\section{Combinatorial Shape and Yields}

The combinatorial PDF is built as a weighted average of the PDFs of the combinatorial events in the sidebands. This is a 2 steps process:

- step 1: evaluation the weighting parameter using the $M C$ dataset bigCombin.

We perform this step using the truth-matched combinatorial candidates from the MC dataset: we extract the PDFs in sidebands (see 5.3.1) then we fit the weighted average of the two PDFs to the combinatorial events in the signal region. The extracted values of the weighting parameters for data are reported in Tab. 5.10.

- step 2: extraction the combinatorial PDFs in the two data sidebands.

In each sideband we first evaluate and fix the signal and misreconstructed-charm PDF using the bigCharm and bigSignal MC datasets, as explained in Sec. 5.3.1, then we extract the combinatorial PDFs.

The combinatorial yields are evaluated using the corrected mass fits results, after subtraction of the misreconstructed-charm component, as explained in Sec. 4.4. The number of combinatorial yields for each decay channel are reported in Tab. 4.9. The untagged $K^{+} K^{-}$channel is treated differently: the combinatorial yields are extracted directly from the simultaneous fit.

The fit procedure for the MC validation fits is very similar to the one for data. Since in $\mathrm{MC}$ we know the number of combinatorial events in the untagged $K^{+} K^{-}$channel, we perform the fit only one time. A second difference is that for each of the cocktails that we fit, cocktailX, we use the corresponding and independent bigSignalX, bigCharmX and bigCombinX MC datasets. A third, and last difference with the data fit, is that the fits in the cocktail sidebands for the untagged $K^{+} K^{-}$channel were unstable and did not converge in some cases, therefore we used the PDF extracted in step 1 to build the weighted average describing the events in the signal region.

\subsubsection{Validation of the Fit on the Simulated Events}

In this section we report the results of the procedure described above on one of our independent cocktails, cocktail1. We show the result of the simultaneous fit run in the nominal configuration, floating the untagged $K^{+} K^{-}$combinatorial yields.

The proper time projections for the fit to cocktail1 are reported in Fig. 5.20. 

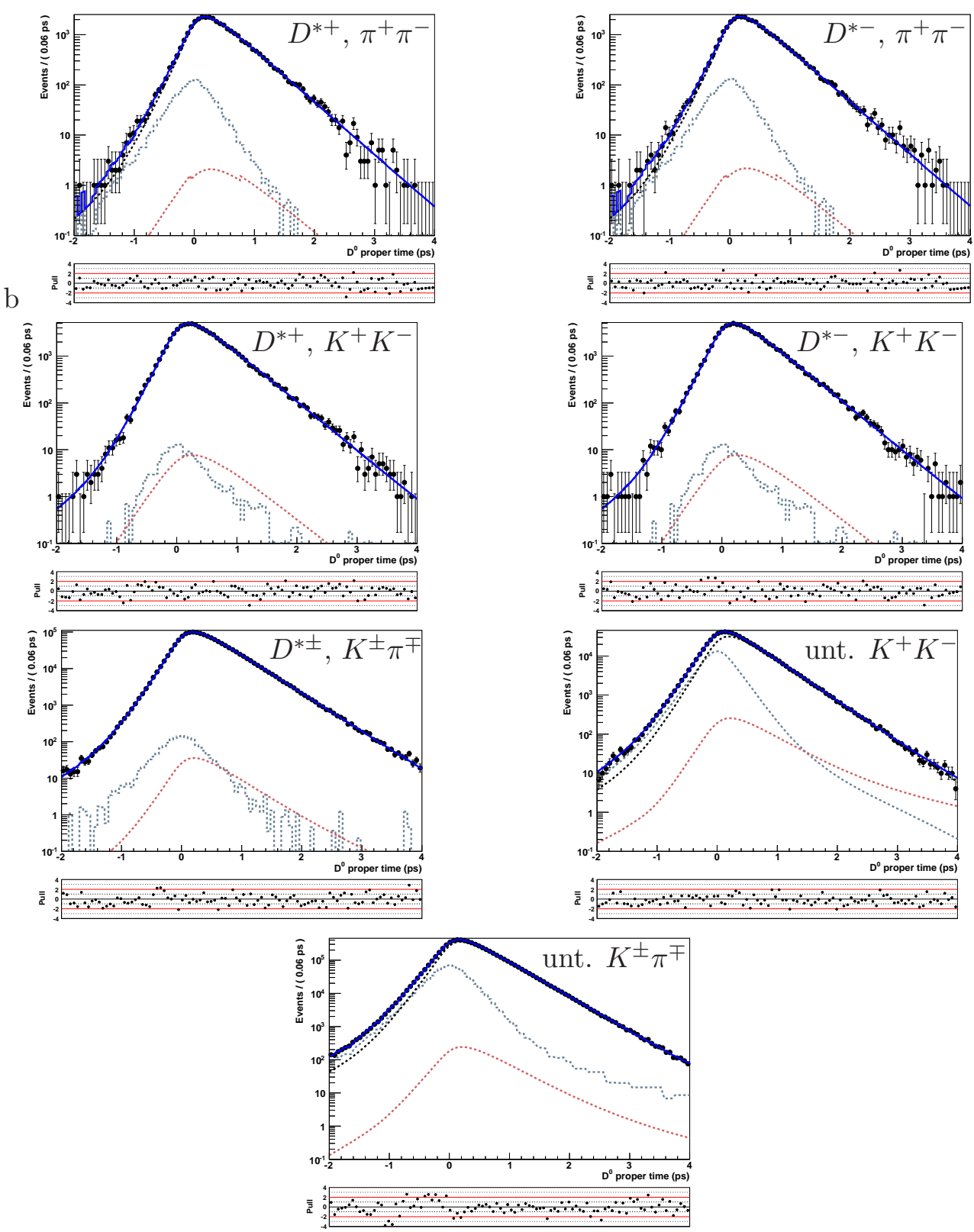

Figure 5.20: Proper time projections of the simultaneous fit to cocktail1 in the nominal configuration, with the untagged $K^{+} K^{-}$combinatorial yields floated. The red dashed line represents the misreconstructed-charm PDF, the blue dashed line the combinatorial one and the black dashed one the signal PDF. 
The extracted value of the resolution function offset is $t_{0}=(-0.34 \pm 0.19) \mathrm{fs}$, while the extracted lifetimes ${ }^{4}$ are:

$$
\begin{aligned}
\tau^{+} & =(411.28 \pm 1.23) \mathrm{fs} \\
\bar{\tau}^{+} & =(412.49 \pm 1.23) \mathrm{fs} \\
\tau_{K \pi} & =(411.71 \pm 0.25) \mathrm{fs},
\end{aligned}
$$

yielding the following values of $y_{C P}$ and $\Delta Y$ :

$$
\begin{aligned}
& y_{C P}=(-0.04 \pm 0.18) \% \\
& \Delta Y=(+0.15 \pm 0.25) \% .
\end{aligned}
$$

All the extracted parameters are compatible with the expectation values within one standard deviation and the total PDF describes well the events in the signal region.

\section{Summary of the Results on the 4 Cocktails}

We repeat the fit to the 4 independent cocktails (see Sec. 4.1), the summary plots on $y_{C P}$, $\Delta Y$, the offset and the lifetimes are reported in Fig. 5.21. We compare two configurations of the fit regarding the untagged $K^{+} K^{-}$combinatorial yields: in one case we fix the untagged $K^{+} K^{-}$combinatorial yields to the true value, in the other we leave it floating. The two configurations yield compatible results, proving that it is possible to extract the number of combinatorial untagged $K^{+} K^{-}$events from the simultaneous fit. The statistical error of $y_{C P}$ increases by $15 \%$ in the configuration where the untagged $K^{+} K^{-}$ combinatorial yields are floating: $0.176 \%$ versus $0.154 \%$. The statistical error on $\Delta Y$, on the other hand, does not change.

\subsubsection{Data Results}

All studies on simulated events do not show any evidence of bias on the observables and we proceed in fitting data. We apply the procedure described in Sec. 5.5.1 and extract the three lifetimes, $\tau^{+}, \bar{\tau}^{+}$and $\tau_{K \pi}$. The proper time projections of the fit to data are reported in Fig. 5.22.

The extracted value of the resolution function offset is $t_{0}=(-2.51 \pm 0.19) \mathrm{fs}$, while the extracted lifetimes are:

$$
\begin{aligned}
\tau^{+} & =(405.69 \pm 1.25) \mathrm{fs} \\
\bar{\tau}^{+} & =(406.40 \pm 1.25) \mathrm{fs} \\
\tau_{K \pi} & =(408.97 \pm 0.24) \mathrm{fs},
\end{aligned}
$$

\footnotetext{
${ }^{4}$ the lifetime input to the MC is $411.67 \mathrm{fs}$.
} 

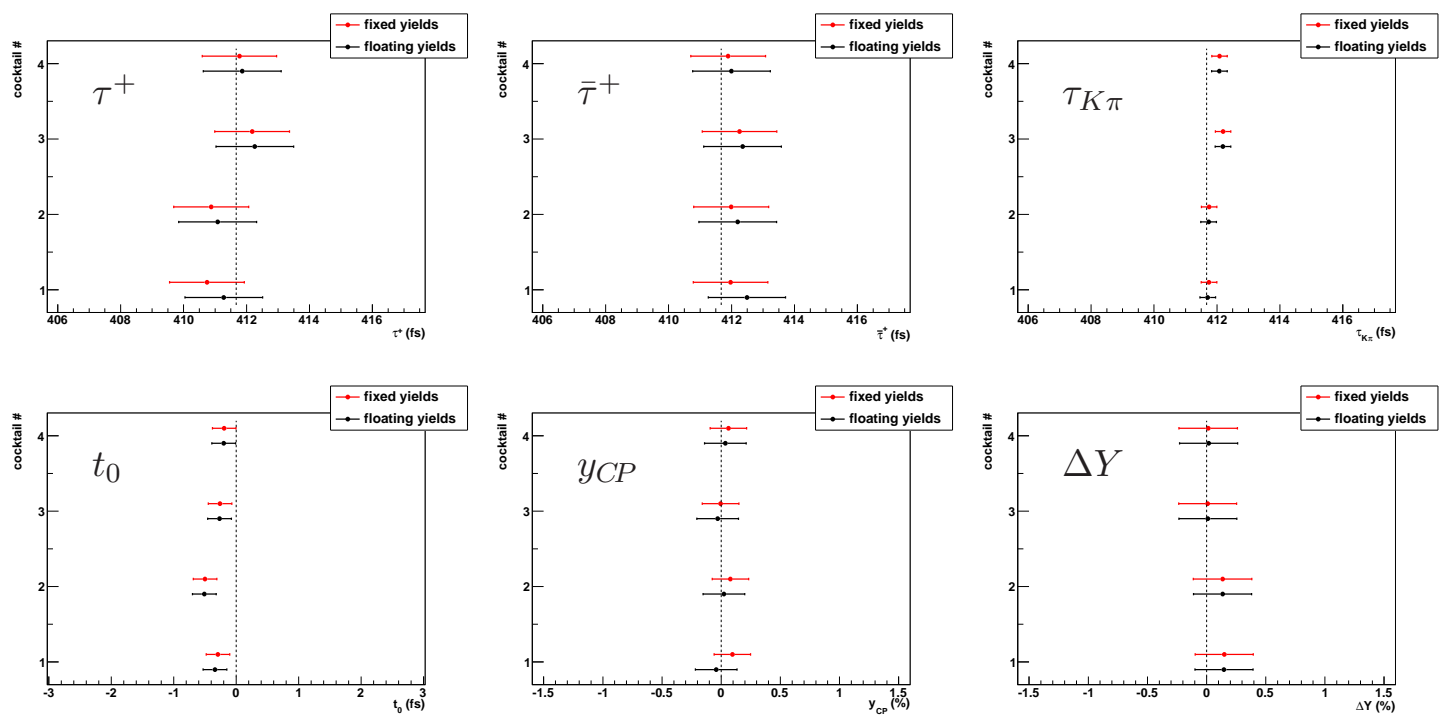

Figure 5.21: Summary of the extracted values of the lifetimes, the resolution function offset and $y_{C P}$ and $\Delta Y$ in the simultaneous fit to the five channels, of the 4 independent $\mathrm{MC}$ cocktails. In red we report the values extracted from the fit where the untagged $K^{+} K^{-}$combinatorial yields are fixed, while in black the fit results where it is floated (the nominal configuration).

yielding the following final values of $y_{C P}$ and $\Delta Y$ :

$$
\begin{aligned}
& y_{C P}=(0.720 \pm 0.180) \% \\
& \Delta Y=(0.088 \pm 0.255) \%,
\end{aligned}
$$

where the error is statistical only and it has been computed using the covariance matrix from the fit. We find no evidence of $C P V$, while the significance of mixing is 4 standard deviation considering the statistical error only.

Unlike the MC fits, the offset extracted from data is significantly different from zero. This is probably a consequence of the misalignment of the SVT, that is perfectly aligned in MC.

The complete list of floated and fixed parameters can be found in Appendix F. In Tab. 5.13 we report the correlations among the lifetimes and the offset. The correlations of the lifetimes with other resolution function parameters are much smaller.

\begin{tabular}{c|cccc}
\hline \hline & $t_{0}$ & $\tau^{+}$ & $\bar{\tau}^{+}$ & $\tau_{K \pi}$ \\
\hline$t_{0}$ & - & & & \\
$\tau^{+}$ & $7.7 \%$ & - & & \\
$\bar{\tau}^{+}$ & $7.7 \%$ & $-35.7 \%$ & - & \\
$\tau_{K \pi}$ & $65.4 \%$ & $5.2 \%$ & $5.2 \%$ & - \\
\hline \hline
\end{tabular}

Table 5.13: Correlation coefficients for the fitted lifetimes and the resolution function offset. 

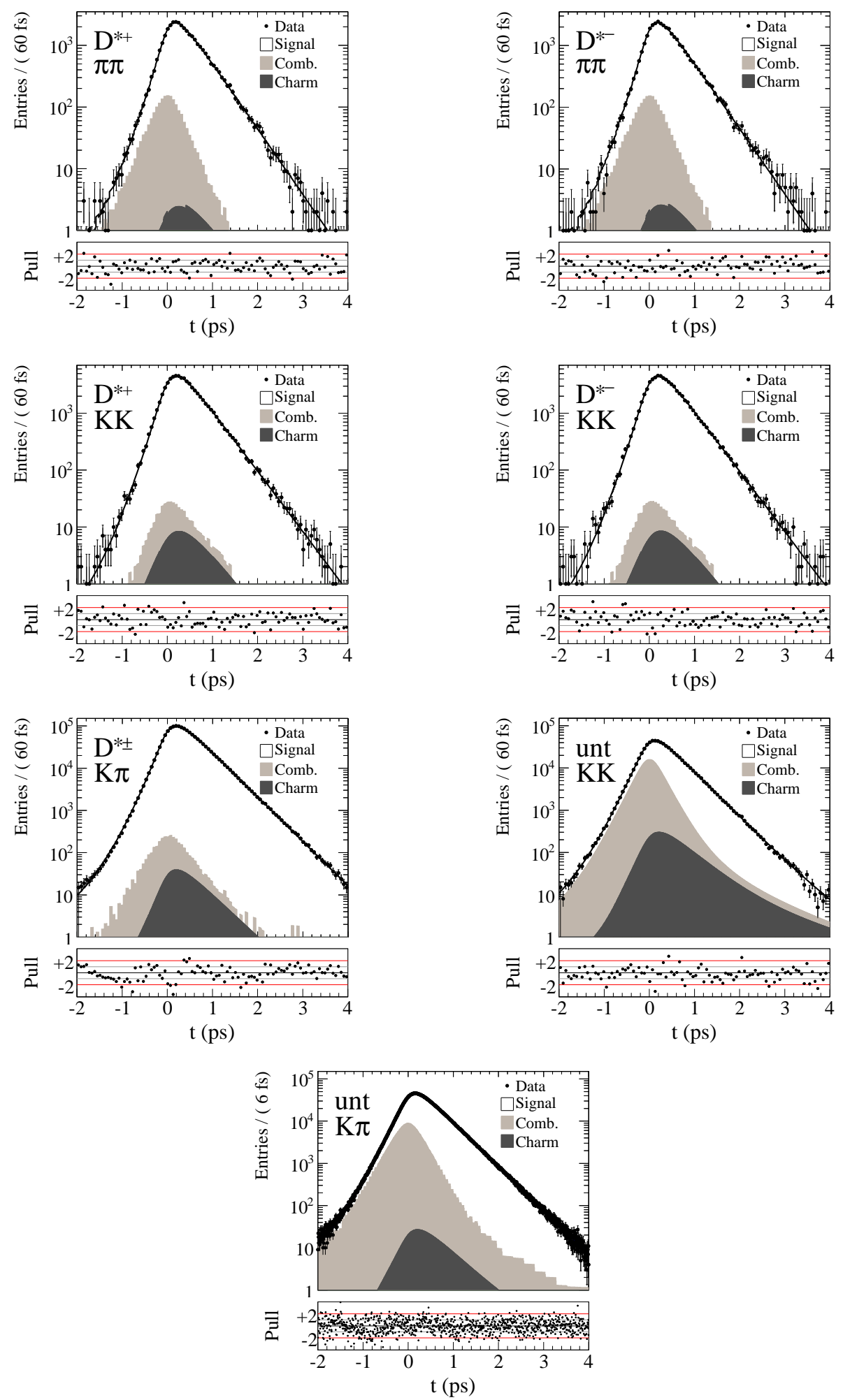

Figure 5.22: Proper time projections of the simultaneous fit to data in the nominal configuration. The combinatorial distribution (indicated as 'Comb.' in light gray) is stacked on top of the misreconstructed-charm distribution (indicated as 'Charm' in dark gray). The normalized Poisson pulls for each fit are shown under each plot; "unt" refers to the untagged datasets. The finer binning in the untagged $K^{ \pm} \pi^{\mp}$ projection was chosen to match the binning of the combinatorial PDF (see Sec. 5.3.1). 
We also check the assumption of symmetric errors, we run MINOS and obtained asymmetric errors, reported in Tab. 5.14, that are very similar the symmetric ones.

\begin{tabular}{c|c|cc}
\hline \hline & $\begin{array}{c}\text { parabolic } \\
\text { error }\end{array}$ & $\begin{array}{c}\text { lower } \\
\text { error }\end{array}$ & $\begin{array}{c}\text { higher } \\
\text { error }\end{array}$ \\
\hline$\tau^{+}(\mathrm{fs})$ & \pm 1.245 & -1.244 & +1.247 \\
$\bar{\tau}^{+}(\mathrm{fs})$ & \pm 1.248 & -1.245 & +1.251 \\
$\tau_{K \pi}(\mathrm{fs})$ & \pm 0.241 & -0.240 & +0.242 \\
\hline \hline
\end{tabular}

Table 5.14: Comparison between parabolic and asymmetric errors on the lifetimes. 


\section{Chapter 6}

\section{Crosschecks and Evaluation of the Systematic Uncertainty}

In this chapter we first present the results of the crosscheck fits performed on data that prove the soundness of the analysis. Then we present the evaluation of the systematic impact of the choice of the signal region, the parameterization of the signal and background events, and the selection criteria. Finally we compare our result with previous $B A B A R$ measurements and analyze their compatibility.

\subsection{Data Crosschecks}

As mentioned in Sec. 3.1.5, we access the values of the three lifetimes only after the validation of the analysis and the determination of the systematic errors, since during the analysis the lifetimes are blinded. Before unblinding we have performed data fits in different configurations to confirm the validity of the analysis. We report in Tab. 6.1 the blinded values of the lifetimes, and also the unblinded resolution function offset, extracted from the nominal blind fit. The three lifetimes have different blinding strings, referred to as BS1, BS2, BS3, that correspond to different blinding values. It is therefore only possible to compare lifetimes with the same blinding string.

\begin{tabular}{l|c|c}
\hline \hline & blinded value $(\mathrm{fs})$ & blinding string \\
\hline$t_{0}$ & $-2.51 \pm 0.19$ & - \\
$\tau^{+}$ & $396.69 \pm 1.25$ & BS1 \\
$\bar{\tau}^{+}$ & $379.07 \pm 1.25$ & BS2 \\
$\tau_{K \pi}$ & $432.23 \pm 0.24$ & BS3 \\
\hline \hline
\end{tabular}

Table 6.1: Blinded lifetime and unblinded offsets extracted from the nominal blind fit to data.

We also present some tests performed after unblinding, and therefore the extracted life- 
times are the real ones.

\subsubsection{Tagged-Only and Untagged-Only Fits}

The first test was to fit separately the tagged and the untagged channels to check whether the lifetimes extracted from the tagged channels are compatible with the ones extracted from the untagged channels.

We fit the three tagged channels alone, as reported in Tab. 6.2, and the two untagged channels alone, as shown in Tab. 6.3. In the untagged-only fit we describe the untagged $K^{+} K^{-}$channel with a single lifetime, as was done in the previous untagged analysis. We associate to that parameter the same blinding string as $\tau^{+}$in the tagged-only fit.

\begin{tabular}{l|c|c}
\hline \hline & blinded value (fs) & blinding string \\
\hline$t_{0}$ & $-3.61 \pm 0.38$ & - \\
tagged $\tau^{+}$ & $395.73 \pm 1.51$ & BS1 \\
tagged $\bar{\tau}^{+}$ & $378.12 \pm 1.51$ & BS2 \\
tagged $\tau_{K \pi}$ & $432.23 \pm 0.51$ & BS3 \\
\hline \hline
\end{tabular}

Table 6.2: Blinded lifetimes and unblinded offset extracted from the tagged-only blind fit to data.

\begin{tabular}{l|c|c}
\hline \hline & blinded value $(\mathrm{fs})$ & blinding string \\
\hline$t_{0}$ & $-2.19 \pm 0.22$ & - \\
untagged $\tau_{K K}$ & $397.95 \pm 0.97$ & $\mathrm{BS} 1$ \\
untagged $\tau_{K \pi}$ & $432.19 \pm 0.28$ & $\mathrm{BS} 3$ \\
\hline \hline
\end{tabular}

Table 6.3: Blinded lifetimes and unblinded offset extracted from the untagged-only blind fit to data.

The offset extracted in the tagged-only fit is $1.4 \mathrm{fs}$ larger in magnitude than the one extracted in the untagged-only fit. The value of the offset, as all the other resolution function parameters, is driven by the most populated mode, in both fits the $K^{ \pm} \pi^{\mp}$ channels. Although the tagged $K^{ \pm} \pi^{\mp}$ channel favors a slightly different offset with respect to the untagged $K^{ \pm} \pi^{\mp}$ channel, the lifetimes are absolutely compatible, and they are compatible also with the extracted lifetime in the nominal blind fit. The untagged $K^{+} K^{-}$lifetime is statistically compatible with $\tau^{+}$, extracted from the tagged-only fit. The extracted value of $\bar{\tau}^{+}$can not be compared to anything since, if we had used the same blinding string as $\tau^{+}$(BS1) we would have had access to the difference of $\tau^{+}$and $\bar{\tau}^{+}$, and therefore to $\Delta Y$.

\subsubsection{Signal PDF Configurations}

In this set of fits to the seven modes we check that the tagged and untagged lifetimes for a given channel, extracted in simultaneous fits with different configurations, are compatible 
within statistical errors.

\section{Channel-Dependent Lifetimes in the Simultaneous Fit}

In the first fit we associate a lifetime parameter to each channel. We fit the $K^{ \pm} \pi^{\mp}$ tagged and untagged samples with separate lifetimes applying the same blinding string. The same is done for the $K^{+} K^{-}$final state: we fit the tagged $D^{0}$ and $\bar{D}^{0}$ decays with a common lifetime (assuming $C P$ conservation), and use the same blinding string for the parameter that describes the untagged $K^{+} K^{-}$decays. The tagged $\pi^{+} \pi^{-} D^{0}$ and $\bar{D}^{0}$ decays are fitted with a common lifetime with different blinding strings. In Tab. 6.4 we report the results of this fit.

\begin{tabular}{l|c|c}
\hline \hline & blinded value $(\mathrm{fs})$ & blinding string \\
\hline$t_{0}(\mathrm{fs})$ & $-2.48 \pm 0.19$ & - \\
tagged $\tau_{\pi \pi}$ & $359.81 \pm 1.86$ & other \\
tagged $\tau_{K K}$ & $396.55 \pm 1.27$ & BS1 \\
untagged $\tau_{K K}$ & $396.62 \pm 0.92$ & BS1 \\
tagged $\tau_{K \pi}$ & $433.13 \pm 0.42$ & BS3 \\
untagged $\tau_{K \pi}$ & $431.99 \pm 0.26$ & BS3 \\
\hline \hline
\end{tabular}

Table 6.4: Blinded lifetimes and unblinded offset extracted from the blind fit to data where each channel is associated with a lifetime parameter.

The tagged and untagged $K^{+} K^{-}$lifetimes are compatible within statistical uncertainties. The $K^{ \pm} \pi^{\mp}$ tagged lifetime is $\sim 1$ fs higher than the untagged one. We explain this behavior with the fact that the tagged $K^{ \pm} \pi^{\mp}$ channel tends to have an offset larger in magnitude than the untagged one, as shown in Tab. 6.2 (tagged-only fit). This effect has no impact on the measurement since the $K^{ \pm} \pi^{\mp}$ lifetimes extracted in the tagged-only fit and in the nominal fit are almost identical.

\section{Tagged and Untagged Lifetimes in the Simultaneous Fit}

Another test configuration is similar to the previous one, except that we describe the tagged $C P$-even channels with the usual $\tau^{+}$and $\bar{\tau}^{+}$. This configuration of the signal PDF was also used to check that the fixed untagged $K^{ \pm} \pi^{\mp}$ combinatorial yields provide an accurate description. We therefore try two sub-configurations for what concerns the untagged $K^{ \pm} \pi^{\mp}$ combinatorial yields: in fit1 it is fixed, as in the nominal fit, in fit2 it is floated, as for the untagged $K^{+} K^{-}$channel. The results of the two fits are reported in Tab. 6.5.

In fit 1 we see that the untagged $K^{+} K^{-}$lifetime is compatible with $\tau^{+}$, and that the tagged $K^{ \pm} \pi^{\mp}$ lifetime is $\sim 1$ fs higher than the untagged one. Therefore fit 1 confirms the results of the previous test. Comparing fit1 with fit2, we observe that the difference of the 


\begin{tabular}{l|c|c|c}
\hline \hline & \multicolumn{2}{|c|}{ blinded value (fs) } & blinding string \\
& fit1 & fit2 & \\
\hline$t_{0}$ & $-2.48 \pm 0.19$ & $-2.40 \pm 0.20$ & - \\
tagged $\tau^{+}$ & $397.19 \pm 1.47$ & $397.27 \pm 1.48$ & BS1 \\
tagged $\bar{\tau}^{+}$ & $379.59 \pm 1.48$ & $379.67 \pm 1.48$ & BS2 \\
untagged $\tau_{K K}$ & $396.65 \pm 0.92$ & $396.65 \pm 0.92$ & BS1 \\
tagged $\tau_{K \pi}$ & $433.13 \pm 0.42$ & $433.21 \pm 0.43$ & BS3 \\
untagged $\tau_{K \pi}$ & $431.99 \pm 0.26$ & $431.93 \pm 0.27$ & BS3 \\
\hline \hline
\end{tabular}

Table 6.5: Blind lifetimes and unblinded offset extracted from the blind fit to data described in the text. In fit1 we fix the untagged $K^{ \pm} \pi^{\mp}$ combinatorial yields to the nominal value, while in fit2 we leave it floating.

extracted lifetimes in the two fits is very small. The extracted number of untagged $K^{ \pm} \pi^{\mp}$ combinatorial events in fit2 is compatible within one standard deviation with the number that we obtain from the mass fit, after the scaling factor correction and the subtraction of the misreconstructed-charm component. This number has been used to evaluate the systematic uncertainty related to the combinatorial yields for the untagged $K^{ \pm} \pi^{\mp}$ channel, as explained later in Sec. 6.2.4.

\section{Modified Signal Resolution Function}

In order to check the dependence of the observables on the resolution model we have performed the fit with a modified version of Eq. 5.3. We have allowed two of the three Gaussians to have a common offset $\left(t_{0}^{\text {add }}\right)$ and the third one (the narrowest one) to have an independent offset $\left(t_{0}\right)$ :

$$
\begin{aligned}
\mathcal{R}\left(t, \sigma_{t} ; s_{i}, t_{0}, \tau\right) & =f_{t 1} \mathcal{D}\left(t, \sigma_{t} ; s_{1}, t_{0}, \tau\right) \\
& +\left(1-f_{t 1}\right)\left[f_{t 2} \mathcal{D}\left(t, \sigma_{t} ; s_{2}, t_{0}^{\text {add }}, \tau\right)\right. \\
& \left.+\left(1-f_{t 2}\right) \mathcal{D}\left(t, \sigma_{t} ; s_{3}, t_{0}^{\text {add }}, \tau\right)\right]
\end{aligned}
$$

The additional offset can be justified by looking at the average value of the reconstructed proper time in bins of $\sigma_{t}$, reported in Fig. 6.1 for the two purest channels: the tagged $K^{ \pm} \pi^{\mp}$ and $K^{+} K^{-}$. Assuming that there is no effect from the background events, the average value of the proper time for $\sigma_{t} \geq 0.15 \mathrm{ps}$ is flat, while for values smaller than $0.15 \mathrm{ps}$ the average value of the proper time decreases when $\sigma_{t}$ increases. This suggests that events with $\sigma_{t}<0.15 \mathrm{ps}$ should have a different offset than the ones with $\sigma_{t}>0.15 \mathrm{ps}$.

This test was performed after unblinding and therefore the extracted lifetimes, reported in Tab. 6.6 with the offset, are unblinded. 

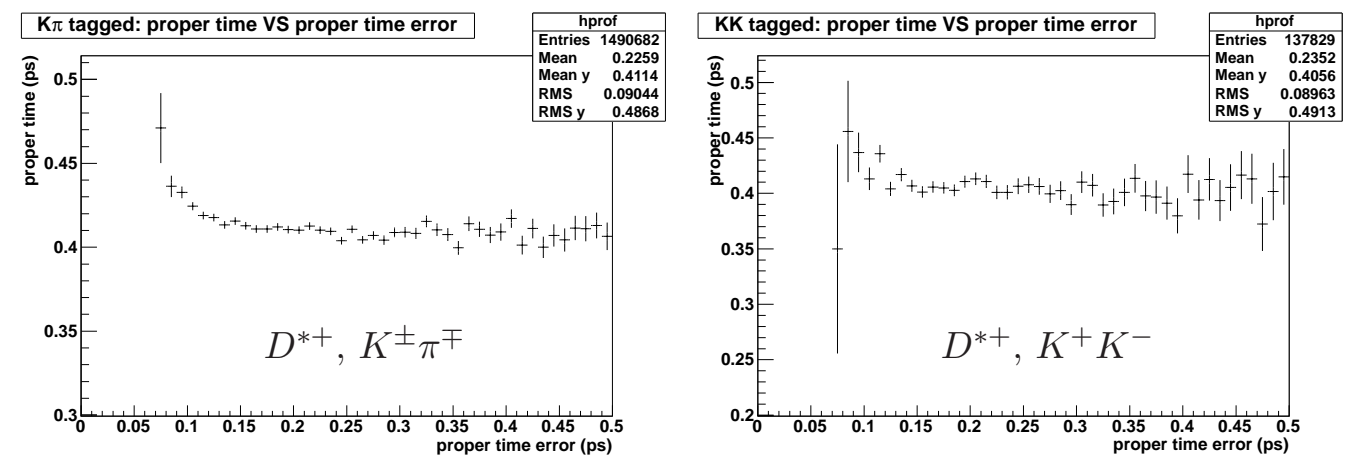

Figure 6.1: Average value of the reconstructed proper time in bins of the proper time error for data events in the signal region.

\begin{tabular}{l|c}
\hline \hline & value $(\mathrm{fs})$ \\
\hline$t_{0}$ & $-4.17 \pm 0.43$ \\
$t_{0}^{\text {add }}$ & $+8.5 \pm 2.5$ \\
$\tau^{+}$ & $406.51 \pm 1.25$ \\
$\bar{\tau}^{+}$ & $407.21 \pm 1.26$ \\
$\tau_{K \pi}$ & $409.77 \pm 0.29$ \\
\hline \hline
\end{tabular}

Table 6.6: Results of the unblind fit to data in the nominal configuration with a modified signal resolution function with two offsets. The fraction of the events fit by the narrowest Gaussian is $81 \%$.

We can also compute $y_{C P}$ and $\Delta Y$ :

$$
\begin{aligned}
& y_{C P}=(0.716 \pm 0.179) \% \\
& \Delta Y=(0.086 \pm 0.254) \% .
\end{aligned}
$$

Although the extracted lifetimes are all higher than the ones extracted in the nominal fit, reported in Eq. 5.18, the impact on $y_{C P}$ and $\Delta Y$ is negligible and we decide not to assign a systematic error.

\subsubsection{Effect of the Direct $C P$ Violation}

In our fit we assume no direct $C P V$ and we fit the tagged $\pi^{+} \pi^{-}$and the tagged $K^{+} K^{-}$ events with the same lifetimes, $\tau^{+}$and $\bar{\tau}^{+}$. As explained in Sec. 1.3 this is the equivalent of neglecting the linear terms in $A_{D}^{h h}$ in Eqs. (1.75) and (1.76).

We repeat the fit releasing this assumption and fitting the two final states with separate lifetimes: $\tau_{K K}^{+}, \bar{\tau}_{K K}^{+}, \tau_{\pi \pi}^{+}$and $\bar{\tau}_{\pi \pi}^{+}$. The untagged $K^{+} K^{-}$channel is fitted with a sum of exponentials, one characterized by $\tau_{K K}^{+}$and the other one by $\bar{\tau}_{K K}^{+}$. This fit was re-run after unblinding, we report the unblinded results in Tab. 6.7.

The results from $K^{+} K^{-}$and $\pi^{+} \pi^{-}$are statistically compatible. Considering the statistical 


\begin{tabular}{l|c|c}
\hline \hline & $\pi^{+} \pi^{-}$ & $K K$ \\
\hline$t_{0}(\mathrm{fs})$ & \multicolumn{2}{|c}{$-2.49 \pm 0.19$} \\
$\tau^{+}(\mathrm{fs})$ & $407.82 \pm 2.60$ & $405.50 \pm 1.46$ \\
$\bar{\tau}^{+}(\mathrm{fs})$ & $409.58 \pm 2.61$ & $405.72 \pm 1.46$ \\
$\tau_{K \pi}(\mathrm{fs})$ & \multicolumn{2}{|c}{$408.98 \pm 0.24$} \\
\hline$y_{C P}(\%)$ & $0.069 \pm 0.456$ & $0.832 \pm 0.194$ \\
$\Delta Y(\%)$ & $0.216 \pm 0.446$ & $0.027 \pm 0.310$ \\
\hline \hline
\end{tabular}

Table 6.7: Lifetimes and offset extracted from a fit where we have released the assumption of no direct $C P V$. The values of $y_{C P}$ and $\Delta Y$ are also reported.

errors on $y_{C P}$ (and $\Delta Y$ ) from $K^{+} K^{-}$and $\pi^{+} \pi^{-}$uncorrelated, we obtain:

$$
\begin{aligned}
& y_{C P}(K K)-y_{C P}(\pi \pi)=(+0.76 \pm 0.50) \% \\
& \Delta Y(K K)-\Delta Y(\pi \pi)=(-0.19 \pm 0.54) \%
\end{aligned}
$$

both compatible with zero.

\subsubsection{Detector Misalignment}

We know that the detector is not perfectly aligned, in particular the SVT sub-detector. In MC this effect is not included. Since we perform a lifetime ratio analysis, at first order we do not expect an effect due to misalignment, because it should cancel in the ratio. In order to check this hypothesis, we have performed the studies described in the following.

\section{Run Dependence}

The alignment of the detector changes over time. We therefore split the dataset in the six running periods, Run1 to Run6, and fit separately the data from each period.

In these fits we have assumed that the shapes of the background categories do not depend on the running period and used the same background PDFs as for the nominal fit. We have rescaled the background yields according to the luminosity of each Run. We could not reevaluate the combinatorial shape from the sidebands in each Run since we use histograms that are affected by large Poisson fluctuations if the bin content is not large enough. We could have changed the binning of the histograms but that would have required a second validation on $\mathrm{MC}$.

Figure 6.2 shows the fitted lifetimes and the values of $y_{C P}$ and $\Delta Y$ from the simultaneous fit to the data sample split into the six Run periods.

No significant variation between Run periods is observed on $y_{C P}$ and $\Delta Y$ and we therefore do not assign a systematic error due to this effect. The lifetimes, especially the $K^{ \pm} \pi^{\mp}$ one, show a variation as a function Run period but this doesn't seem to have an effect our 

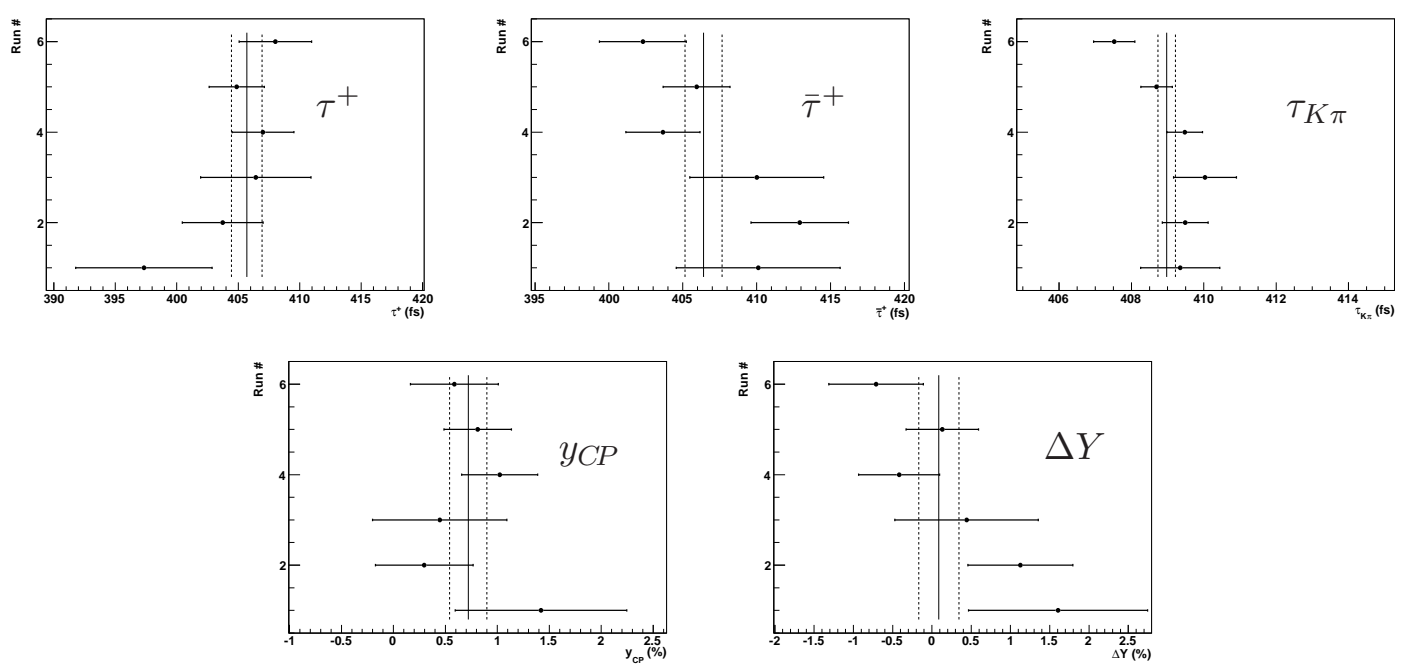

Figure 6.2: Extracted lifetimes values of $y_{C P}$ and $\Delta Y$ in each Run period. The solid line represent the value extracted in the nominal fit and the dotted lines represent the $\pm 1 \sigma$ region.

observables.

\section{Dependence on the $D^{0}$ Azimuthal Angle}

The SVT alignment also depends on the geometry. The first three layers of the SVT, the most important for tracking, consist in six modules parallel to the beam direction. Therefore the effect of the SVT misalignment can be checked by studying the azimuthal dependence of the lifetimes.

In order to qualitatively study the dependence on the $D^{0}$ azimuthal angle $\phi$ in the laboratory frame we have produced profile plots of the quantity $\left(t+t_{0}\right) / \tau$, where $t_{0}$ and $\tau$ are the fitted offset and lifetime from the nominal fit. The plots, reported in Fig. 6.3, have been produced for data (and signal MC) only for the purest tagged $K^{ \pm} \pi^{\mp}$ and $K^{+} K^{-}$ channels, where the background is almost negligible.
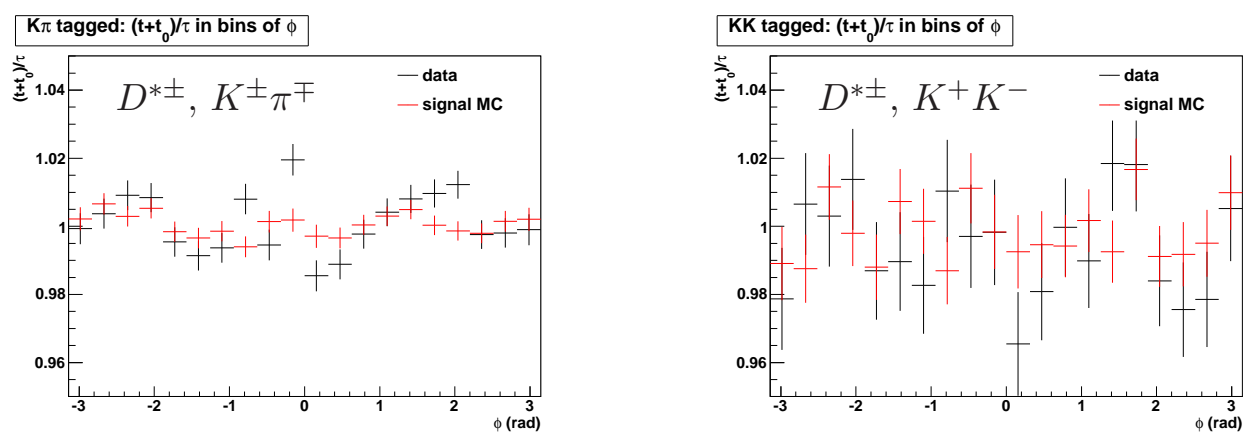

Figure 6.3: Value of the quantity $\left(t+t_{0}\right) / \tau$ in bins of the $D^{0}$ azimuthal angle in the laboratory frame. 
In the tagged $K^{+} K^{-}$plots we do no appreciate any systematic dependence, indeed, fitting the plots with a constant we find that the extracted value was compatible with 1 with probabilities above $30 \%$. On the other hand, in the tagged $K^{ \pm} \pi^{\mp}$ channel we do observe an oscillation that was also noted in the previous analyses. Performing the fit in bins of $\phi$, it was shown that the effect was absorbed in the offset and that there was not a residual effect on the lifetimes nor on $y_{C P}$ and $\Delta Y$.

Repeating the whole simultaneous fit in bins of $\phi$ would present the problems highlighted in the Run-split fits, related to the low statistics bins of the combinatorial PDFs. However, unlike that case, the dependence of the background shapes with the angles is more evident and assuming that we can use the nominal background PDFs to describe the background in bins of the angle is not allowed.

Although in the previous analysis no systematic effect was shown when performing the fit in bins of the angles, we study the tagged $K^{ \pm} \pi^{\mp}$ lifetime $\phi$-dependence. We perform extended unbinned ML fits to the proper time and proper time error distributions of the tagged $K^{ \pm} \pi^{\mp}$ decays in six bins of $\phi$. In this channel the background shapes in the different bins are compatible with the integrated shape, so we have used the same PDFs as in the nominal fit to describe the backgrounds. The background yields have been estimated from MC. The distribution of the proper time error for signal events has been re-evaluated in each bin. The fitted lifetime and offset are reported in Fig. 6.4 for the fits in bins of $\phi$.
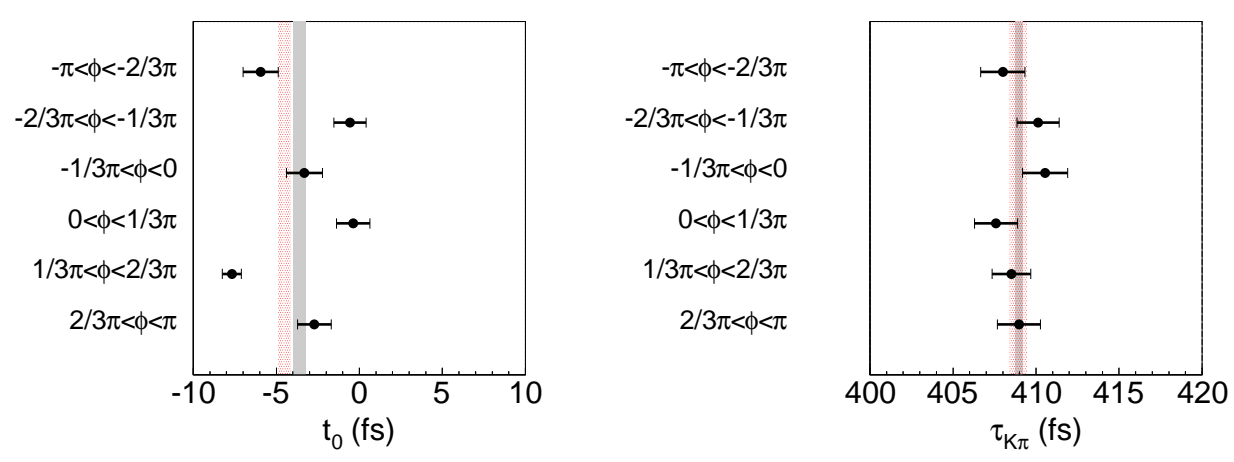

Figure 6.4: Fitted offset (left) and lifetime (right) in bins of $\phi$ for the tagged $K^{ \pm} \pi^{\mp}$ channel. The gray band represents the value from the nominal fit $\pm 1 \sigma$ while the red band shows the weighted average $\pm 1 \sigma$.

The offset is greatly dependent on the angle while the lifetime is much more stable, suggesting that the offset absorbs the $\phi$-dependent variations, that do not affect the lifetime. The weighted average of the 6 independent measurements in $\phi$ is $\tau=(408.93 \pm 0.52)$ fs and the $\chi^{2}$ is 4 for 5 degrees of freedom, yielding a probability of $55 \%$ for the 6 values to be compatible with the average. We therefore do not observe a systematic effect, the oscillations are compatible within the statistical error and the average is in good agreement with the value of the lifetime extracted in the nominal fit (see Eq. 5.18). 


\section{Dependence on the $D^{0}$ Polar Angle in the CM}

At first order we do not expect any impact of misalignment depending on the $D^{0}$ polar angle. At the CHARM2012 Conference [56] Belle presented its preliminary result for the measurement of $y_{C P}$ using the tagged channels only. They performed the measurement in bins of cosine of the polar angle in the $\mathrm{CM}, \cos \theta^{*}$. One reason was that Belle replaced its inner vertex detector after a few years of operation, with another one with more layers. A second reason is that their data-MC agreement of the profile plots of $\left(t+t_{0}\right) / \tau$ was very poor. The reason why they use the angle in the CM and not in the laboratory resides in the fact that they do not distinguish between data taken at the $\Upsilon(1 S), \Upsilon(2 S), \Upsilon(3 S)$, $\Upsilon(4 S)$ or $\Upsilon(5 S)$ resonances. To homogenize the procedure they perform the analysis in the $\mathrm{CM}$ so that the differences that would be evident in the laboratory disappear.
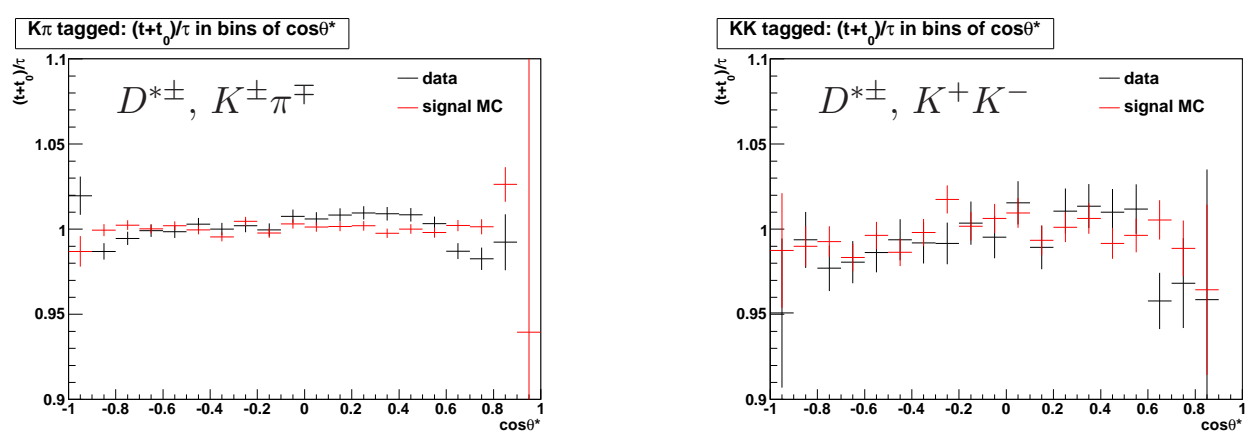

Figure 6.5: Profile plots of the quantity $\left(t+t_{0}\right) / \tau$ in bins of the cosine of the polar angle of the $D^{0}$ in the CM.

After Belle presented their results we studied the $\cos \theta^{*}$ dependence as we have done for the $\phi$-dependence. Looking at Fig. 6.5, as for the azimuthal angle, there seems to be a more evident systematic oscillation in the $K^{ \pm} \pi^{\mp}$ mode. This behavior was also observed and checked in the previous analysis, and no systematic impact on $y_{C P}$ and $\Delta Y$ was assigned. Nonetheless we repeated the fits to the $K \pi$ tagged channel only, shown in Fig. 6.6.
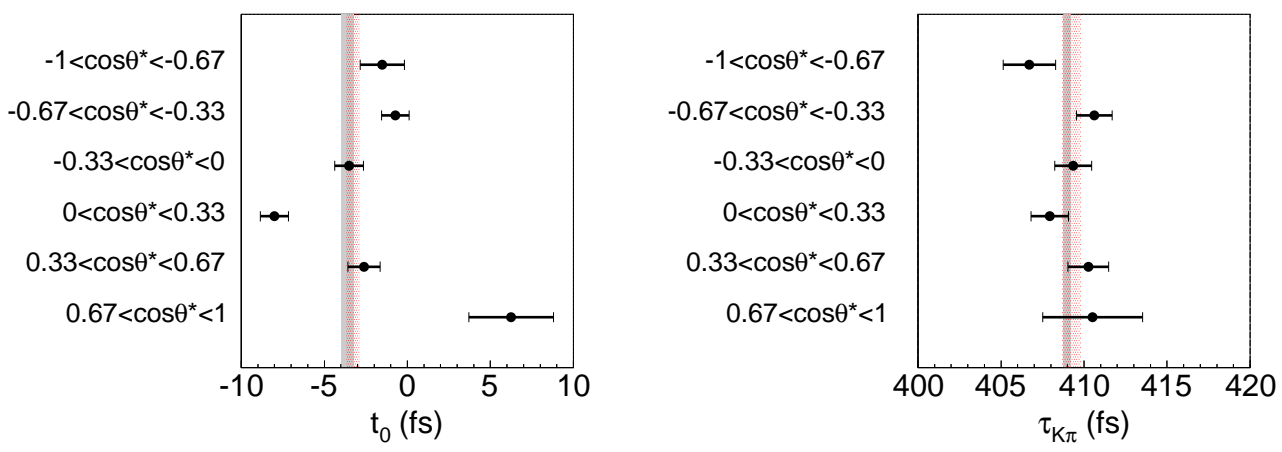

Figure 6.6: Fitted offset (left) and lifetime (right) in bins of $\cos \theta^{*}$ for the tagged $K^{ \pm} \pi^{\mp}$ channel. The gray band represents the value from the nominal fit $\pm 1 \sigma$ while the red band shows the weighted average $\pm 1 \sigma$. 
Again, as for $\phi$, the extracted offset varies significantly for different values of the angle while the lifetime is much more stable. The weighted average of the 6 independent measurements in $\cos \theta^{*}$ is $\tau=(409.25 \pm 0.52)$ fs and the $\chi^{2}$ in this case is 6.5 for 5 degrees of freedom, yielding a probability of $26 \%$ for the 6 values to be compatible with the average. In this case the average is slightly higher that the value extracted in the nominal fit.

These tests are not completely exhaustive because we only measured the effect on the $D^{0}$ lifetime, and not on the effective lifetimes $\tau^{+}$and $\bar{\tau}^{+}$. Moreover, as we have seen in the modified resolution function test, in Tab. 6.6, systematic shifts in the lifetimes do not imply a systematic error on the observable. Finally, this test has been performed after unblinding and it was not possible to change the analysis anymore. In the previous $B A B A R$ analysis the fit was performed in bins of $\cos \theta$ (polar angle in the laboratory) and $\phi$ and no systematic impact on $y_{C P}$ and $\Delta Y$ was observed. We therefore decided not to assign a systematic error.

\subsubsection{Additional Crosschecks}

The extracted value of $y_{C P}$ is lower than the previous $B A B A R$ measurement, reported in Tab. 3.1. At the end of this chapter we present a more quantitative comparison. In the following we present the fits performed in order to understand the effect of the main differences between the two analysis.

\section{Simultaneous Fit with Definition of the Signal Regions of the Previous Anal- yses}

In order to understand the effect of the signal region optimization performed in this analysis, we have repeated the fit implementing the signal region definition of the previous analyses:

$$
1.8495 \mathrm{GeV} / c^{2}<m_{D^{0}}<1.8795 \mathrm{GeV} / c^{2} \quad \text { for the tagged channels }
$$

and

$$
1.8545 \mathrm{GeV} / c^{2}<m_{D^{0}}<1.8745 \mathrm{GeV} / c^{2} \quad \text { for the untagged channels, }
$$

the $\Delta m$ region definitions is the same.

The results are reported in Tab. 6.8.

The effect is very small on $\Delta Y$, while it is of the order of $0.13 \%$ for $y_{C P}$. The effect on $y_{C P}$ is due to a change in the $K \pi$ lifetimes (and also in the offset), while the $C P$-even lifetimes do not change significantly. The offset is $\sim 0.6 \mathrm{fs}$ larger in magnitude in the previous analysis, suggesting that we are better centering the signal region around the mass peaks and the effect of the correlation between mass and proper time is at least reduced. Since we have performed an optimization of the signal region, we do not believe 


\begin{tabular}{l|c|c}
\hline \hline & old definition & new definition \\
\hline$t_{0}(\mathrm{fs})$ & $-3.11 \pm 0.19$ & $-2.51 \pm 0.19$ \\
$\tau^{+}(\mathrm{fs})$ & $405.64 \pm 1.26$ & $405.69 \pm 1.25$ \\
$\bar{\tau}^{+}(\mathrm{fs})$ & $406.21 \pm 1.26$ & $406.40 \pm 1.25$ \\
$\tau_{K \pi}(\mathrm{fs})$ & $409.36 \pm 0.24$ & $408.97 \pm 0.24$ \\
\hline$y_{C P}(\%)$ & $0.85 \pm 0.18$ & $0.72 \pm 0.18$ \\
$\Delta Y(\%)$ & $0.07 \pm 0.26$ & $0.09 \pm 0.26$ \\
\hline \hline
\end{tabular}

Table 6.8: Comparison between the nominal fit values (new definition) and the ones obtained when using the definition of the signal region implemented in the previous analyses (old definition).

that the difference in $y_{C P}$ and $\Delta Y$ between fits performed with the new and old signal region definition is a good estimation of the systematic error associated with the choice of the signal region. Its evaluation is described later in Sec. 6.2.1.

\section{Simultaneous Fit to the Previous Dataset}

Another important difference between this and the previous analyses is the fitting procedure: the fit strategy and the PDFs definition. In order to estimate the impact of this difference, we apply our fit procedure to exactly the same datasets (data and MC) used in the previous analyses and repeat the tagged-only and untagged-only measurements. We have also performed the simultaneous fit to both the tagged and untagged channels. We have adopted the signal and sideband regions defined of the previous analysis. The combinatorial and misreconstructed-charm background yields have been taken from the previous analysis, we have not performed mass fits.

The new results compared to the old results are reported in Tab. 6.9 for the tagged-only fit, in Tab. 6.10 for the untagged-only fit, and in Tab. 6.11 for the simultaneous fit.

\begin{tabular}{l|c|c}
\hline \hline & old procedure & new procedure \\
\hline$t_{0}(\mathrm{fs})$ & $-4.75 \pm 0.51$ & $-4.76 \pm 0.51$ \\
tagged $\tau^{+}(\mathrm{fs})$ & $403.28 \pm 2.07$ & $403.21 \pm 2.07$ \\
tagged $\bar{\tau}^{+}(\mathrm{fs})$ & $405.34 \pm 2.11$ & $405.29 \pm 2.11$ \\
tagged $\tau_{K \pi}(\mathrm{fs})$ & $409.33 \pm 0.70$ & $409.39 \pm 0.70$ \\
\hline$y_{C P}(\%)$ & $1.24 \pm 0.39$ & $1.27 \pm 0.39$ \\
$\Delta Y(\%)$ & $0.26 \pm 0.36$ & $0.26 \pm 0.36$ \\
\hline \hline
\end{tabular}

Table 6.9: Comparison between the tagged-only fits to the dataset used in the previous tagged analysis with the new and old procedure.

The tagged-only fits with the new and old procedure yields very similar results on the lifetimes, and consequently on $y_{C P}$ and $\Delta Y$. The untagged-only fit with the new procedure yields a $\sim 20 \%$ lower value of $y_{C P}$ compared to the value obtained with the old procedure. 


\begin{tabular}{l|c|c}
\hline \hline & old & new \\
\hline$t_{0}(\mathrm{fs})$ & $-5.67 \pm 0.28$ & $-5.06 \pm 0.29$ \\
untagged $\tau_{K^{+} K^{-}}(\mathrm{fs})$ & $405.84 \pm 1.00$ & $406.60 \pm 1.31$ \\
untagged $\tau_{K \pi}(\mathrm{fs})$ & $410.39 \pm 0.38$ & $410.27 \pm 0.38$ \\
\hline$y_{C P}(\%)$ & $1.12 \pm 0.26$ & $0.90 \pm 0.34$ \\
\hline \hline
\end{tabular}

Table 6.10: Comparison between the untagged-only fits to the dataset used in the previous untagged analysis with the new and old procedure.

\begin{tabular}{l|c|c}
\hline \hline & old & new \\
\hline$t_{0}(\mathrm{fs})$ & - & $-4.95 \pm 0.25$ \\
$\tau^{+}(\mathrm{fs})$ & - & $404.31 \pm 1.73$ \\
$\bar{\tau}^{+}(\mathrm{fs})$ & - & $406.43 \pm 1.74$ \\
$\tau_{K \pi}(\mathrm{fs})$ & - & $410.10 \pm 0.33$ \\
\hline$y_{C P}(\%)$ & $1.16 \pm 0.22$ & $1.17 \pm 0.24$ \\
$\Delta Y(\%)$ & - & $0.26 \pm 0.36$ \\
\hline \hline
\end{tabular}

Table 6.11: Comparison between the combined (tagged+untagged) old result and the simultaneous fit to the dataset used in the previous analyses with the new procedure.

The $K^{ \pm} \pi^{\mp}$ lifetime is very similar in the old and new fits, while the $K^{+} K^{-}$lifetime differs by $\sim 0.8 \mathrm{fs}$. We also notice that the statistical error on $y_{C P}$ extracted with the new procedure is $\sim 30 \%$ larger than the one obtained in the previous analysis. This effect is due to the fact that the expected number of combinatorial events in the untagged $K^{+} K^{-}$mode is left floating in the new procedure. The simultaneous fit to the tagged and untagged channel yields a value of $y_{C P}$, reported in Tab. 6.11, that is almost identical to the combined value of the previous tagged and untagged analyses, obtained with the BLUE method [52]. Therefore we conclude that, in the simultaneous fit to the five channels, the difference in the procedures does not produce a significant difference in the central values of the observables $y_{C P}$ and $\Delta Y$.

\subsection{Evaluation of the Systematic Uncertainty}

In this analysis we make a certain number of experimental assumptions. The evaluation of their systematic impact on $y_{C P}$ and $\Delta Y$ is reported in the next sections.

\subsubsection{Fit Region}

The choice of the fit region was the result of a data-driven optimization, as described in Sec. 4.3. Nonetheless we decide to study the possible systematic effects related to the position and width of the signal regions. We expect a reduction of the error with respect to the previous analyses, since no optimization was done before. 


\section{Signal Region Position}

The position of the signal region is determined by the parameter $m_{C}$, its center. In the nominal fit we subdivide the five channels in two groups, within each group the center of the signal region is shared. The $K^{+} K^{-}$tagged and untagged channels are centered at $1864.215 \mathrm{MeV} / c^{2}$, the other three channel are centered $\sim 170 \mathrm{keV} / c^{2}$ below.

In order to estimate the systematic error associated with the position of the signal region, we redo the fit centering the signal region of each channel at the estimated peak center (see Tab. 4.3). The background shape and yields are not re-evaluated since the shift is small. The systematic errors is taken as the variation of the two observables, that is very small, as reported in Tab. 6.12. The offset of the resolution function does not change significantly.

\begin{tabular}{c|c|c|c}
\hline \hline position of & $\Delta\left[y_{C P}\right]$ & $\Delta[\Delta Y]$ & $\Delta\left[t_{0}\right]$ \\
signal region & $(\%)$ & $(\%)$ & $(\mathrm{fs})$ \\
\hline independent centers & -0.005 & +0.001 & +0.00 \\
\hline \hline
\end{tabular}

Table 6.12: Variation of $y_{C P}$ and $\Delta Y$ obtained by centering the signal region of each channel in the estimated peak center (see Tab. 4.3) and redoing the fit. The change in the offset is also reported.

\section{Signal Region Width}

To estimate the systematic uncertainty on $y_{C P}$ and $\Delta Y$ due to the choice of the width of the $D^{0}$ mass signal region, we alter the size of the signal region by \pm 4 and $\pm 2 \mathrm{MeV} / c^{2}$ and re-fit the data, in each case keeping the signal region center obtained from the optimization. In all cases, we re-fit the MC to determine the proper time distribution and the yield of misreconstructed-charm backgrounds in the new signal region and the weighting parameter for the combinatorial PDF. We also re-evaluate the scaling factors $s_{m}$ for the correction of the background yields in the signal region. The changes are very small. We also re-build the $\sigma_{t}$ histogram for signal events. For each variation, we compute the change in $y_{C P}$ and $\Delta Y$ from the nominal fit, the results are reported in Tab. 6.13.

This test is meant to account for the effect of the correlation between mass and proper time. However, different signal regions widths present different background levels and it is not possible to disentangle this effect from the one coming from the correlation. The value of the resolution function offset is very stable, with variations of the order of $10^{-2}$ of fs. This suggests that the correlation between mass and proper time is under control thanks to the optimization of the signal region and that the systematic impact on $y_{C P}$ and $\Delta Y$ comes from the different background levels. The systematic error associated with the expected number of background events in the signal region is evaluated in a different way. Nevertheless, we conservatively keep this systematic error, evaluating it as the RMS 


\begin{tabular}{c|c|c|c}
\hline \hline $\begin{array}{c}\text { change } \\
\text { in width }\end{array}$ & $\begin{array}{c}\Delta\left[y_{C P}\right] \\
(\%)\end{array}$ & $\begin{array}{c}\Delta[\Delta Y] \\
(\%)\end{array}$ & $\begin{array}{c}\Delta\left[t_{0}\right] \\
(\mathrm{fs})\end{array}$ \\
\hline$+4 \mathrm{MeV} / c^{2}$ & -0.012 & +0.033 & -0.02 \\
$+2 \mathrm{MeV} / c^{2}$ & +0.023 & +0.012 & +0.00 \\
$-2 \mathrm{MeV} / c^{2}$ & +0.070 & +0.021 & +0.03 \\
$-4 \mathrm{MeV} / c^{2}$ & +0.086 & -0.018 & +0.04 \\
\hline $\mathrm{RMS} / 2$ & 0.057 & 0.022 & - \\
\hline \hline
\end{tabular}

Table 6.13: The change in $y_{C P}$ and $\Delta Y$ from the nominal fit to data, as a function of the change in the width of the signal region. The change in the resolution offsets is also reported, its statistical error is $0.19 \mathrm{fs}$.

of the 4 values and dividing it by $\sqrt{4}$.

\subsubsection{Signal PDF}

The observed proper time distribution for signal events is constructed by convolving a resolution function with an exponential and it is reported in Sec. 5.1.1 for the seven modes. In the nominal fit we have estimated the number of correctly reconstructed $D^{0}$ (and $\bar{D}^{0}$ ), combined with a misidentified slow pion (the mistagged signal events) using MC events. We have also assumed that the untagged $K^{+} K^{-}$channel was composed half of $D^{0}$ and half of $\bar{D}^{0}$. In the construction of the $\sigma_{t}$ PDF in the untagged $K^{+} K^{-}$channel: we assume that the number of combinatorial events extracted from a first fit to data is correctly describing the events in the signal region.

In the following we evaluate the systematic impact of these assumptions on $y_{C P}$ and $\Delta Y$.

\section{Signal Proper Time Error PDF of the Untagged $K^{+} K^{-}$channel}

The signal proper time error PDF consists of a histogram and it is built by subtracting the misreconstructed-charm and combinatorial contributions from the $\sigma_{t}$ distribution of the events in the signal region. This operation requires the knowledge of the number of expected events of the two background categories. In all the channels except the untagged $K^{+} K^{-}$the number of combinatorial events is known, since it is extracted from the mass fits. In the untagged $K^{+} K^{-}$channel, on the other hand, we extract it during the fit and therefore we need an estimate to build the proper time error histogram. In the nominal fit we have performed the fit a first time and then used the extracted number of combinatorial events to re-build the proper time error. We have then redone the fit a second time and consider this one as the nominal fit.

In order to evaluate the systematic error we have repeated the fit a third time and taken as a systematic error the difference between the nominal result and the one that come from this third fit. The result is reported in Tab. 6.14. The extracted number of combinatorial 
events is 164740 compared to 164972 of the nominal fit, the statistical error on both numbers is 1000 . The difference between the starting extracted values is reduced by almost a factor 6 with respect to the second iteration of the fit.

\begin{tabular}{c|c|c|c}
\hline \hline $\begin{array}{c}\text { signal PDF } \\
\text { variation }\end{array}$ & $\begin{array}{c}\Delta\left[y_{C P}\right] \\
(\%)\end{array}$ & $\begin{array}{c}\Delta[\Delta Y] \\
(\%)\end{array}$ & $\begin{array}{c}\Delta\left[t_{0}\right] \\
(\mathrm{fs})\end{array}$ \\
\hline untagged $K^{+} K^{-} \sigma_{t}$ PDF & +0.022 & +0.000 & +0.01 \\
\hline \hline
\end{tabular}

Table 6.14: The change in $y_{C P}$ and $\Delta Y$ obtained with the third iteration of the fit after rebuilding the proper time error histogram of the untagged $K^{+} K^{-}$signal events.

\section{Fraction of Mistagged Signal Events in the Tagged $C P$-even Channels}

In the tagged $K^{+} K^{-}$and $\pi^{+} \pi^{-}$channels we estimate, using simulated events, that the fraction correctly reconstructed $D^{0}$ (and $\bar{D}^{0}$ ) associated with a misidentified pion with the wrong charge is $0.2 \%$. This fraction $f_{\text {tag }}^{ \pm}$is fixed in the signal PDF. To estimate the systematic error associated with this assumption we apply a relative variation on $f_{\mathrm{tag}}^{ \pm}$of $\pm 20 \%$ but observe no significant variation on $\Delta Y$ and $y_{C P}$.

\section{Fraction of $D^{0}$ Events in the Untagged $K^{+} K^{-}$Channel}

In the nominal fit we assume that the untagged $K^{+} K^{-}$channel is composed half of $D^{0}$ and half of $\bar{D}^{0}$ decays. We evaluate the systematic error associated with this assumption by varying the relative amount of $D^{0} \rightarrow K^{+} K^{-}$and $\bar{D}^{0} \rightarrow K^{+} K^{-}$decays. The average value over the 9 independent signal-only MC datasets is $50.12 \%$, with an RMS of $0.07 \%$. The MC does not take into account $C P V$, the most precise measurement to date is the CDF one [57], that reports an integrated asymmetry for the $D^{0} \rightarrow K^{+} K^{-}$channel of:

$$
A_{C P}=(-0.24 \pm 0.22 \pm 0.09) \%
$$

in terms of $D^{0}$ fraction this corresponded to $f_{D^{0}}=\frac{1}{2}\left(A_{C P}+1\right)=49.88 \%$.

In order to take into account both the sources of systematics we vary the fraction of $D^{0}$ candidates in the untagged $K^{+} K^{-}$channel by $\pm 0.12 \%$. The results are reported in Tab. 6.15.

\begin{tabular}{l|c|c}
\hline \hline fraction of $D^{0}$ in & $\Delta\left[y_{C P}\right]$ & $\Delta[\Delta Y]$ \\
untagged $K^{+} K^{-}$ & $(\%)$ & $(\%)$ \\
\hline $50.12 \%$ & +0.000 & +0.00 \\
$49.88 \%$ & +0.001 & +0.00 \\
\hline \hline
\end{tabular}

Table 6.15: The change in $y_{C P}$ and $\Delta Y$ obtained by changing the fraction $D^{0}$ candidates in the untagged $K^{+} K^{-}$channel by $\pm 0.12 \%$. The nominal value of this faction is $50 \%$. 


\subsubsection{Misreconstructed-Charm PDF and Yields}

The expected number of events and the PDF of the misreconstructed-charm background in the signal region is evaluated by fitting the truth-matched events of the bigCharm MC dataset. The $\mathrm{MC}$ dataset is equivalent to 10 times the integrated luminosity of data, hence the statistical error of the parameters is reduced with respect to the previous analyses. There is still a systematic component that we have to evaluate, related to the assumption that the MC misreconstructed-charm events correctly reproduce data.

\section{Misreconstructed-Charm Yields}

To evaluate the systematic uncertainty associated to the number of misreconstructedcharm events fixed in the final fit, we conservatively vary the expected charm yield by $\pm 10 \%$ in the tagged channels and $\pm 5 \%$ in the untagged ones. These correspond to more than 2 statistical standard deviations in all channels. We apply the variation to all the channels simultaneously, in other words, we increase (and then decrease) the number of misreconstructed-charm events in each of the 5 decay channels and we repeat the fit. The results of these variations are shown in Table 6.16.

\section{Misreconstructed-Charm Lifetime}

For the shape of the misreconstructed-charm background, we conservatively vary the lifetime ${ }^{1}$ in the PDF by $\pm 5 \%$ for all the samples except tagged $\pi^{+} \pi^{-}$, where the variation is $\pm 15 \%$ since the statistical error is higher. Also in this case the variations correspond to more than 2 standard deviations. As for the yields we apply the variation simultaneously to all the channels. The results of these variations are shown in Table 6.16.

\begin{tabular}{l|c|c|c}
\hline \hline change charm & $\Delta\left[y_{C P}\right]$ & $\Delta[\Delta Y]$ & $\Delta\left[t_{0}\right]$ \\
lifetime and yields & $(\%)$ & $(\%)$ & $(\mathrm{fs})$ \\
\hline scale up $N_{\text {crm }}$ & +0.016 & +0.000 & +0.00 \\
scale down $N_{\text {crm }}$ & -0.016 & +0.000 & +0.00 \\
\hline scale up $\tau_{\text {crm }}$ & +0.042 & -0.001 & +0.00 \\
scale down $\tau_{\text {crm }}$ & -0.040 & +0.000 & +0.01 \\
\hline \hline
\end{tabular}

Table 6.16: The change in $y_{C P}$ and $\Delta Y$ as a function of the variation in the yields $\left(N_{\mathrm{crm}}\right)$ and lifetimes $\left(\tau_{\text {crm }}\right)$ of the misreconstructed-charm background.

\subsubsection{Combinatorial PDF and Yields}

The combinatorial PDF for the events in the signal region is determined by fitting the data in the sideband regions and then building the weighted average of the PDFs extracted in

\footnotetext{
${ }^{1}$ when there are two lifetimes, we vary both of them.
} 
the sidebands. The expected number of combinatorial events are estimated with mass fits and then fixed (except for the untagged $K^{+} K^{-}$channel). We consider variation in the yields and in the weighting parameters. We also consider the systematic error associated with the extrapolation of the combinatorial PDFs in the two sidebands since the signal and the misreconstructed-charm component there are estimated from MC.

\section{Combinatorial Yields}

We fix the expected number of combinatorial events to the ones obtained with the mass fit, after a applying a correction rescaling factor and after subtracting the misreconstructedcharm component. Both the rescaling factor $s_{m}$ and the misreconstructed-charm yields are taken from MC. The untagged $K^{+} K^{-}$channel is an exception, we do not fix the combinatorial yields but we extract it from the fit, therefore we do not consider this channel in this systematic.

In order to evaluate the systematics assigned to tagged combinatorial yields we have repeated the fits varying the scale factor $s_{m}$ estimated on MC. The amount of variation is $15 \%$ of the quantity $1-s_{m}$, that correspond to the disagreement between the fit and the truth yields. This variation corresponds to a variation of $4.5 \%$ of the combinatorial yields for the tagged $\pi^{+} \pi^{-}, 15 \%$ for the $K^{+} K^{-}$and $20 \%$ for the $K^{ \pm} \pi^{\mp}$. We repeat the fit applying the variation to the 3 tagged channels simultaneously and take as a systematic error the difference with the nominal fit values, reported in Tab. 6.17.

For the untagged $K^{ \pm} \pi^{\mp}$ channel we have adopted another procedure. We use the number of combinatorial events extracted from fit2 of the crosscheck reported in Sec. 6.1.2, Tab. 6.5. We repeat the fit fixing the combinatorial yields to the number extracted in fit2. The results are reported in Tab. 6.17.

\begin{tabular}{l|r|r|c}
\hline \hline change combin. & \multicolumn{1}{|c|}{$\begin{array}{c}\Delta\left[y_{C P}\right] \\
(\%)\end{array}$} & $\begin{array}{c}\Delta[\Delta Y] \\
\text { yields }\end{array}$ & $\begin{array}{c}\Delta\left[t_{0}\right] \\
(\mathrm{fs})\end{array}$ \\
\hline scale up tagged yields & -0.028 & -0.002 & -0.02 \\
scale down tagged yields & +0.030 & -0.001 & +0.03 \\
\hline untagged $K^{ \pm} \pi^{\mp}$ yields & -0.031 & +0.000 & +0.08 \\
\hline Total & 0.043 & 0.002 & - \\
\hline \hline
\end{tabular}

Table 6.17: The change in $y_{C P}$ and $\Delta Y$ as a function of the change in the combinatorial yields. The total is obtained adding in quadrature the largest contributions from the variation of the tagged yields to the contribution of the variation of the untagged $K^{ \pm} \pi^{\mp}$ yields.

\section{Combinatorial Weighting Parameter}

The weighting parameter is evaluated with an unbinned ML fit to the MC combinatorial events in the signal region. In order to evaluate the systematic error associated to this 
assumption we vary $h$ for each channel according to its statistical error. If the RMS of the values of $h$ extracted from the four cocktail MC samples is larger than the statistical error, then we use the former as amount of variation. The results are reported in Tab. 6.18.

\begin{tabular}{l|c|c|c}
\hline \hline change combin. & $\Delta\left[y_{C P}\right]$ & $\Delta[\Delta Y]$ & $\Delta\left[t_{0}\right]$ \\
weight. param. & $(\%)$ & $(\%)$ & $(\mathrm{fs})$ \\
\hline scale up $h$ & +0.003 & -0.001 & +0.10 \\
scale down $h$ & -0.004 & +0.000 & -0.10 \\
\hline \hline
\end{tabular}

Table 6.18: The change in $y_{C P}$ and $\Delta Y$ as a function of the change in the weighting parameter of the combinatorial PDFs.

\section{Extraction of the Combinatorial PDF from the Sidebands}

The combinatorial PDF is extracted from the sidebands, after fixing the signal and misreconstructed-charm contributions, evaluated on MC. We check for possible systematic effects of the assumptions on the signal and misreconstructed-charm components.

We vary the misreconstructed-charm yields and lifetime in the sidebands as we have done in the signal region (Sec. 6.2.3) and extract each time the combinatorial PDFs in the sidebands following the nominal procedure. Each time we redo the simultaneous fit and evaluate the change in $y_{C P}$ and $\Delta Y$, the results are reported in Tab. 6.19. We also vary the signal yields by the same fraction used for the misreconstructed-charm ones. In addition we vary the signal lifetimes in the sidebands shifting their value by the statistical error of the lifetime used to correct the value extracted from MC (see Eq. 5.12).

\begin{tabular}{l|c|c|c}
\hline \hline change combin. & $\Delta\left[y_{C P}\right]$ & $\Delta[\Delta Y]$ & $\Delta\left[t_{0}\right]$ \\
PDFs in sidebands & $(\%)$ & $(\%)$ & $(\mathrm{fs})$ \\
\hline scale up $\tau_{\text {crm }}$ & -0.059 & +0.000 & +0.01 \\
scale down $\tau_{\text {crm }}$ & +0.062 & +0.000 & +0.00 \\
\hline scale up $N_{\text {crm }}$ & -0.022 & +0.000 & +0.00 \\
scale down $N_{\text {crm }}$ & +0.023 & +0.000 & +0.00 \\
\hline shift up $\tau_{\text {sig }}$ & +0.000 & +0.000 & +0.00 \\
shift down $\tau_{\text {sig }}$ & +0.001 & +0.000 & +0.00 \\
\hline scale up $N_{\text {sig }}$ & -0.001 & +0.000 & +0.00 \\
scale down $N_{\text {sig }}$ & +0.002 & +0.000 & +0.00 \\
\hline Total & 0.066 & 0.000 & - \\
\hline \hline
\end{tabular}

Table 6.19: The change in $y_{C P}$ and $\Delta Y$ a function of the change in the yields and lifetimes of the misreconstructed-charm and signal PDFs in the sidebands. The total is obtained adding in quadrature the largest contributions for each group of variations. 


\subsubsection{Event Selection}

Selection criteria systematics are evaluated by applying our fit procedure to the data samples obtained by varying different selection cuts. Since there are changes to the data sample, there is a statistical component associated with this systematic, but it not possible to disentangle it.

\section{Proper Time Error Selection}

We change the proper time error range to $\sigma<0.4 \mathrm{ps}$ and $\sigma<0.6 \mathrm{ps}$ instead of the nominal cut $\sigma<0.5 \mathrm{ps}$. In these fits we have repeated the mass fits only for data, therefore we have used the nominal value for the scaling factor $s_{m}$ for the total background yields. The results of these variations are shown in Table 6.20. As done for the variations of the signal region width, the systematic error associated is computed as the RMS of the 2 values and dividing it by $\sqrt{2}$.

\begin{tabular}{l|c|c|c}
\hline \hline change in & $\Delta\left[y_{C P}\right]$ & $\Delta[\Delta Y]$ & $\Delta\left[t_{0}\right]$ \\
selection & $(\%)$ & $(\%)$ & $(\mathrm{fs})$ \\
\hline$\sigma_{t}<0.4 \mathrm{ps}$ & -0.060 & -0.069 & -0.20 \\
$\sigma_{t}<0.6 \mathrm{ps}$ & +0.043 & -0.028 & -0.08 \\
\hline $\mathrm{RMS} / \sqrt{2}$ & +0.052 & +0.053 & - \\
\hline \hline
\end{tabular}

Table 6.20: The change in $y_{C P}$ and $\Delta Y$ a function of the change in the $\sigma_{t}$ selection criteria.

\section{Adjudication Selection}

Essentially, if a $D^{0} / D^{*}$ candidate shares one or more tracks with another $D^{0} / D^{*}$ in the same sample, the one with the highest vertex $\chi^{2}$ probability is retained. We intended to repeat the fits and consider two variations: keep all overlapping candidates or rejecting all overlapping candidates. Unfortunately time constrains didn't allow us to perform these fits. We have evaluated the systematic error associated the adjudication procedure using the results of the previous analyses. We apply the same systematic variation evaluated in the previous tagged analysis, reported in Tab. 6.21. The systematic error on $y_{C P}$ in the untagged analysis was smaller.

\begin{tabular}{l|c|c|c}
\hline \hline change in & $\Delta\left[y_{C P}\right]$ & $\Delta[\Delta Y]$ & $\Delta\left[t_{0}\right]$ \\
selection & $(\%)$ & $(\%)$ & $(\mathrm{fs})$ \\
\hline keep all candidates & +0.024 & -0.003 & - \\
reject all candidates & -0.028 & +0.011 & - \\
\hline \hline
\end{tabular}

Table 6.21: The change in $y_{C P}$ and $\Delta Y$ a function of the change in the adjudication of multiple candidates. The errors are taken from the previous tagged analysis. 


\subsubsection{Systematic Summary}

The effects of systematic variations on $y_{C P}$ are shown in Table 6.22. In this Table each entry is the largest relative to the variation corresponding to the row. The total systematic error is obtained summing in quadrature each contribution.

\begin{tabular}{cl|c|c}
\hline \hline Category & Fit Variation & $\left|\Delta\left[y_{C P}\right]\right|(\%)$ & $|\Delta[\Delta Y]|(\%)$ \\
\hline \multirow{2}{*}{ Fit Region } & position of signal region & 0.005 & 0.001 \\
& width of signal region & 0.057 & 0.022 \\
\hline \multirow{3}{*}{ Signal } & untagged $K^{+} K^{-} \sigma_{t}$ signal PDF & 0.022 & 0.000 \\
& mistag fraction & 0.000 & 0.000 \\
& $D^{0}$ fraction in untagged $K^{+} K^{-}$ & 0.001 & 0.000 \\
\hline \multirow{2}{*}{ Charm } & yields & 0.016 & 0.000 \\
& lifetimes & 0.042 & 0.001 \\
\hline \multirow{2}{*}{ Combinatorial } & yields & 0.043 & 0.002 \\
& weighting parameter & 0.004 & 0.001 \\
& PDF from sidebands & 0.066 & 0.000 \\
\hline \multirow{2}{*}{ Selection } & $\sigma_{t}$ cut & 0.052 & 0.053 \\
& adjudication & 0.028 & 0.011 \\
\hline \multicolumn{2}{l}{} & 0.124 & 0.058 \\
\hline \hline
\end{tabular}

Table 6.22: Effects of systematic variations on $y_{C P}$ and $\Delta Y$. The total systematic error reported in the last row is obtained summing in quadrature each contribution listed in this table.

\subsection{Compatibility with the Previous BABAR Results}

The current and the previous $B A B A R$ measurements of $y_{C P}$ and $\triangle Y$ are reported in Tab. 6.23.

\begin{tabular}{c|c|c}
\hline \hline & previous & current \\
\hline$y_{C P}$ & $(1.16 \pm 0.22 \pm 0.18) \%$ & $(0.72 \pm 0.18 \pm 0.12) \%$ \\
$\Delta Y$ & $(0.26 \pm 0.36 \pm 0.08) \%$ & $(0.09 \pm 0.26 \pm 0.06) \%$ \\
\hline \hline
\end{tabular}

Table 6.23: Summary of the previous and the current measured values of $y_{C P}$ and $\Delta Y$ at $B A B A R$. The first error is statistical and the second systematic. The sign of $\Delta Y$ of the previous measurement has been changed in order to have compatible definitions.

In order to judge whether the previous and current measurements of the observables are statistically compatible or not, we evaluate the probability of the minimized $\chi^{2}(\lambda)$. The value of $\lambda$ that minimizes $\chi^{2}, \hat{\lambda}$, is the most probable value of the observable given the 
two measurements. For each observable, $y_{C P}$ and $\Delta Y$, the $\chi^{2}(\lambda)$ is given by:

$$
\chi^{2}(\lambda)=\sum_{i, j=1}^{2}\left(x_{i}-\lambda\right)\left(V^{-1}\right)_{i j}\left(x_{j}-\lambda\right)
$$

where $i, j$ run over the measurements, $x_{i}$ is the measured value of the observable and $V$ is the covariance matrix. The covariance matrix has a statistical and a systematic contribution $V=V_{\text {stat }}+V_{\text {syst }}$, each contribution is of the form:

$$
V_{i j}=\rho_{i j} \sigma_{i} \sigma_{j}
$$

where $\rho_{i j}$ is the correlation between the two errors $\sigma_{i}$ and $\sigma_{j}$, therefore $\rho_{11}=\rho_{22}=1$ and $\rho_{21}=\rho_{12}=\rho$.

The statistical correlation $\rho_{\text {stat }}$ is defined to be:

$$
\rho_{\text {stat }}=\frac{N_{\text {overlap }}}{\sqrt{N_{\text {new }} N_{\text {old }}}}
$$

where $N_{\text {overlap }}$ is the number of events in common of the two data samples and $N_{\text {new }}\left(N_{\text {old }}\right)$ is the number of events in the current (previous) data sample. Looking at the timestamp of each event we have evaluated $N_{\text {new }}, N_{\text {old }}$ and $N_{\text {overlap }}$ for the five channels separately and also for all three tagged channels together and the two untagged channels together. These numbers are reported in Tabs. 6.24 and 6.25. This procedure does not take into account the fact that an event may contain multiple candidates.

\begin{tabular}{l|c|c|c|c}
\hline \hline channel & $N_{\text {old }}$ & $N_{\text {new }}$ & $N_{\text {overlap }}$ & $\rho_{\text {stat }}$ \\
\hline tagged $\pi^{+} \pi^{-}$ & 31283 & 69112 & 28611 & $61.5 \%$ \\
tagged $K^{+} K^{-}$ & 69949 & 137818 & 63142 & $64.3 \%$ \\
tagged $K^{ \pm} \pi^{\mp}$ & 735192 & 1489328 & 669396 & $64.0 \%$ \\
\hline all tagged & 836296 & 1695644 & 761058 & $63.9 \%$ \\
\hline \hline
\end{tabular}

Table 6.24: Number of events in the previous $\left(N_{\text {old }}\right)$ and current $\left(N_{\text {new }}\right)$ data sample, along with number of common events $\left(N_{\text {overlap }}\right)$ and the statistical correlation $\left(\rho_{\text {stat }}\right)$ for the tagged channels. In the last row the evaluation has been made not distinguishing the different channels.

The last rows of Tabs 6.24 and 6.25 report the statistical correlation for the tagged-only and the untagged-only channels. The statistical correlation when all the five channels are involved is computed summing the correspondent numbers of events in the last rows of the tables and then using Eq. (6.10). The statistical correlation of the previous and current data samples is $\rho_{\text {stat }}=62.0 \%$. 


\begin{tabular}{l|c|c|c|c}
\hline \hline channel & $N_{\text {old }}$ & $N_{\text {new }}$ & $N_{\text {overlap }}$ & $\rho_{\text {stat }}$ \\
\hline untagged $K^{+} K^{-}$ & 325974 & 666653 & 297085 & $63.7 \%$ \\
untagged $K^{ \pm} \pi^{\mp}$ & 2873611 & 6856826 & 2664812 & $60.0 \%$ \\
\hline all untagged & 3198076 & 7515468 & 2960707 & $60.4 \%$ \\
\hline \hline
\end{tabular}

Table 6.25: Number of events in the previous $\left(N_{\text {old }}\right)$ and current $\left(N_{\text {new }}\right)$ data sample, along with number of common events $\left(N_{\text {overlap }}\right)$ and the statistical correlation $\left(\rho_{\text {stat }}\right)$ for the untagged channels. In the last row the evaluation has been made not distinguishing the different channels.

The correlation among the systematic errors is very hard to estimate, therefore we study two cases:

- systematic errors fully correlated: $\rho_{\text {syst }}=100 \%$;

- systematic errors correlated as much as the statistical errors: $\rho_{\text {syst }}=\rho_{\text {stat }}=62 \%$.

Finally, we minimize the $\chi^{2}(\lambda)$ of Eq. (6.8) for the two observables and report the values of $\hat{\lambda}$, the $\chi^{2}$ and the probability in Tab. 6.26.

\begin{tabular}{r|ccc|ccc}
\hline \hline \multirow{2}{*}{$\rho_{\text {syst }}$} & \multicolumn{3}{|c|}{$y_{C P}$} & \multicolumn{3}{c}{$\Delta Y$} \\
& $\hat{\lambda}$ & $\chi^{2}$ & $P\left(\chi^{2}\right)$ & $\hat{\lambda}$ & $\chi^{2}$ & $P\left(\chi^{2}\right)$ \\
\hline $100 \%$ & 0.728 & 5.5 & $1.9 \%$ & 0.098 & 0.40 & $52.8 \%$ \\
$62 \%$ & 0.795 & 3.7 & $5.3 \%$ & 0.102 & 0.38 & $53.7 \%$ \\
\hline \hline
\end{tabular}

Table 6.26: Values of $\hat{\lambda}$, minimized $\chi^{2}$ and associated probability $P\left(\chi^{2}\right)$, computed considering one degree of freedom, that the previous and the current measurements are statistically compatible. Two values for correlation of the systematic errors $\left(\rho_{\text {syst }}\right)$ have been investigated: complete correlation and same correlation as the statistical errors.

As expected, the $\Delta Y$ measurements are perfectly compatible. The most conservative approach gives a compatibility of around $2 \%$ between the previous $y_{C P}$ result and the current.

The dataset used in the previous analysis is not completely included in the new dataset: $9 \%$ (7.4\%) of the tagged (untagged) events are excluded. Since we have a looser selection, we can explain this fact as a consequence of the reprocessing (see Sec 2.9) of the BABAR data. Improved particle reconstruction and identification algorithms have been implemented for the new dataset and as a consequence a fraction of events that passed the selection in the previous analyses is excluded from the new dataset. In principle we could combine the previous and current measurements. However, the number of excluded events is quite small, and, in addition, the events are excluded as a consequence of an improvement in reconstruction. Therefore we do not combine the measurements, the results of this analysis supersede the previous $B A B A R$ results. 
As shown in Sec. 6.1.5 the fit using the new procedure on the old dataset yields a very similar result, therefore the source of the discrepancy between the previous and current $y_{C P}$ measurement should not be the result of different procedures and fitting machinery, but most probably in the datasets themselves. We list the possible sources of differences between the previous and the current datasets:

- change of the signal region definition: data-driven optimization of the position and width;

- integrated luminosity increased by $20 \%$ : addition of Run6;

- change in the selection criteria: removal of the helicity cut and change of PID selector of the tracks to improve the statistics;

- BABAR data reprocessing with improved particle reconstruction and improved particle identification; MC has been reproduced;

From the studies presented in this chapter we can estimate the impact of the first two differences. As shown in Sec. 6.1.5 the optimization of the signal region has an effect of $-0.13 \%$ on $y_{C P}$, the compatibility with the previous result rises up to $9.9 \%(17.3 \%)$ considering the systematic errors $100 \%$ (62\%) correlated. The value of $y_{C P}$ extracted from Run6 only is lower with respect to the weighted average excluding it, as shown in Fig. 6.2. The statistical error affecting the Run6-only measurement prevents us from drawing a precise quantitative conclusion as in the previous case. We can estimate the effect of the addition of Run6 as the difference between the nominal $y_{C P}$ value and the Run1 to Run5 weighted average. We conclude that the effect is around half of the one estimated for the signal region and it is in the same direction.

The other two differences have not been investigated, due to time constrains, but we have a partial explanation for the low compatibility of the previous and the current measurements of $y_{C P}$. 



\section{Chapter 7}

\section{Conclusions}

In this final chapter we summarize the results and we discuss the impact of the measurement on the current experimental situation.

The analysis and the final result presented in this thesis have been reported in a journal paper accepted for publication on Physical Review D [58].

\subsection{Final Result}

We use the full $\Upsilon(4 S)$ BABAR dataset, corresponding to $468 \mathrm{fb}^{-1}$, and measure the effective lifetimes of the $D^{0}\left(\tau^{+}\right)$and the $\bar{D}^{0}\left(\bar{\tau}^{+}\right)$decaying to the $C P$-even eigenstates $K^{+} K^{-}$and $\pi^{+} \pi^{-}$, and the lifetime of the flavor eigenstates in the $C P$-mixed final state $K^{ \pm} \pi^{\mp}\left(\tau_{K \pi}\right)$ :

$$
\begin{aligned}
\tau^{+} & =(405.69 \pm 1.25) \mathrm{fs} \\
\bar{\tau}^{+} & =(406.40 \pm 1.25) \mathrm{fs} \\
\tau_{K \pi} & =(408.97 \pm 0.24) \mathrm{fs},
\end{aligned}
$$

where the error is only statistical. A representative plot that visually shows the differences of the lifetimes is reported in Fig. 7.1.

We combine these lifetimes following

$$
\begin{aligned}
& y_{C P}=\frac{\tau_{K \pi}}{2}\left[\frac{1}{\tau^{+}}+\frac{1}{\bar{\tau}^{+}}\right]-1 \\
& \Delta Y=\frac{\tau_{K \pi}}{2}\left[\frac{1}{\tau^{+}}-\frac{1}{\bar{\tau}^{+}}\right]
\end{aligned}
$$

and obtain the mixing parameter $y_{C P}$ and the $C P$-violating parameter $\Delta Y$ :

$$
\begin{aligned}
& y_{C P}=(0.72 \pm 0.18 \pm 0.12) \% \\
& \Delta Y=(0.09 \pm 0.26 \pm 0.06) \%
\end{aligned}
$$




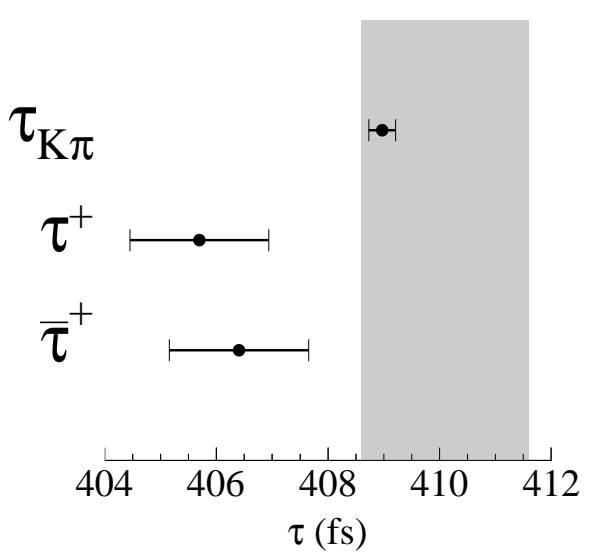

Figure 7.1: The lifetimes extracted from the simultaneous fit (with statistical uncertainties only). The gray band indicates the PDG $D^{0}$ lifetime $\pm 1 \sigma[23]$.

where the first error is statistical and the second is systematic. We exclude the no-mixing hypothesis with a $3.3 \sigma$ significance and find no evidence of $C P$ violation.

This represents the most precise single measurement of $y_{C P}$ and favors a lower value of the parameter with respect to the previous measurements. The central value of the world weighted average of the published $y_{C P}$ measurements decreases by $\sim 25 \%$ if we include this measurement and exclude the previous BABAR one. This measurement contributes to improve the overall precision on $y_{C P}$ and $\Delta Y$ : the world average errors decrease by $\sim 15 \%$ if we include this measurement and exclude the previous BABAR one. In Fig. 7.2 we report two plots that compare how the central value and error on $y_{C P}$ and $A_{\Gamma}$ change with the new measurement.

We plot $A_{\Gamma}$ instead of $\Delta Y$ because the other experiments have chosen this parameter to describe $C P V$, the two parameters are related by: $\Delta Y=A_{\Gamma}\left(1+y_{C P}\right)$.

The measured value of $y_{C P}$ is compatible with the value of the mixing parameter $y_{\mathrm{D}}$ obtained with a direct measurement in a time-dependent Dalitz Plot analysis on the full BABAR $\Upsilon(4 S)$ statistics [59]:

$$
\begin{aligned}
& x_{\mathrm{D}}=(0.16 \pm 0.23 \pm 0.12 \pm 0.08) \%, \\
& y_{\mathrm{D}}=(0.57 \pm 0.20 \pm 0.13 \pm 0.07) \%
\end{aligned}
$$

where the first error is statistical, the second is the experimental systematic and the third is a systematic error related to the Dalitz Plot model. We also show the measured value of $x_{\mathrm{D}}$ for completeness. The two parameters $y_{\mathrm{D}}$ and $y_{C P}$ are expected to have the same value in absence of $C P V$. 

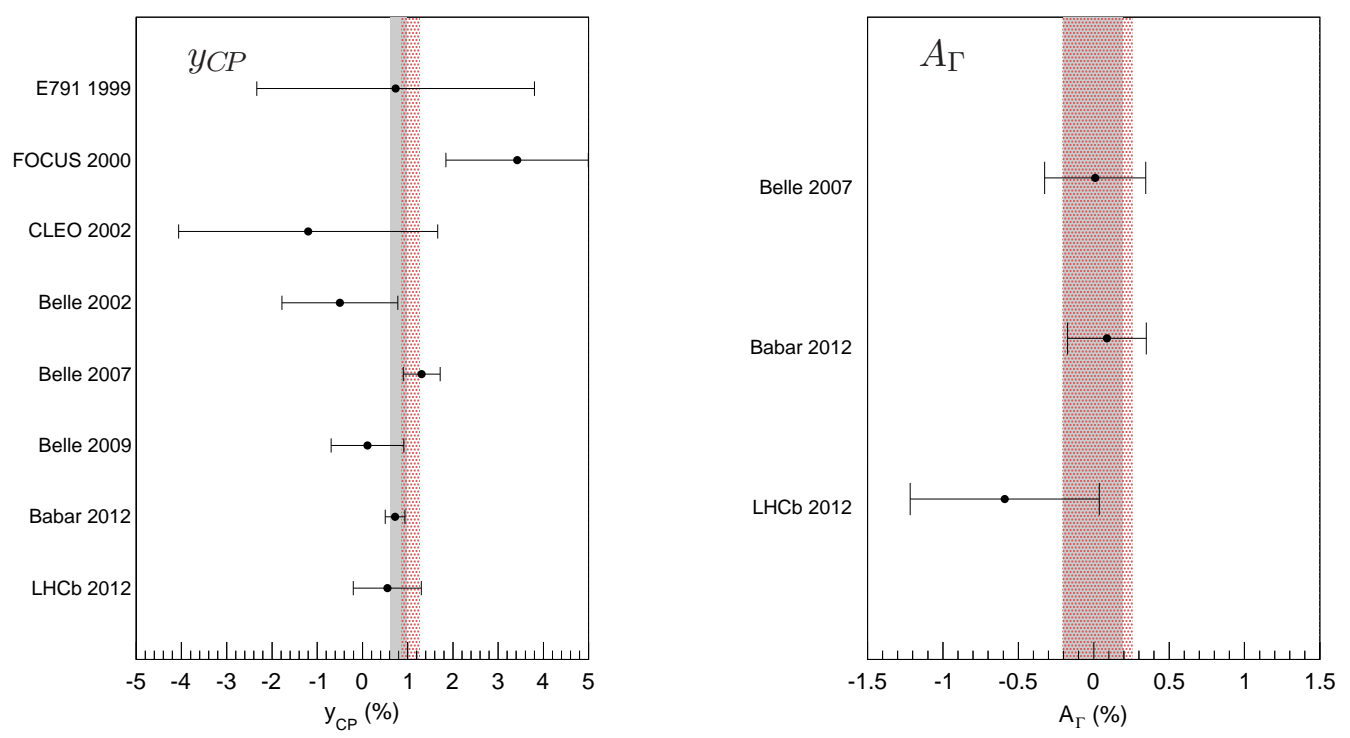

Figure 7.2: World weighted average of the $y_{C P}$ (left) and $A_{\Gamma}$ (right) measurements. The red band indicates the average $\pm 1 \sigma$ excluding this measurement, the gray band has been computed replacing the previous BABAR measurement with this one.

\section{Belle Preliminary Results}

In May 2012, at the Charm2012 Conference [56], Belle presented the preliminary result of the measurement of $y_{C P}$ using the tagged channels only, with $976 \mathrm{fb}^{-1}$ of data collected primarily at the $\Upsilon(4 S)$, but also at $\Upsilon(1 S), \Upsilon(2 S), \Upsilon(3 S)$, and $\Upsilon(5 S)$ resonances.

They obtain:

$$
\begin{aligned}
y_{C P} & =(+1.11 \pm 0.22 \pm 0.11) \% \\
A_{\Gamma} & =(-0.03 \pm 0.20 \pm 0.08) \% .
\end{aligned}
$$

They exclude the no-mixing hypothesis at $4.5 \sigma$ significance and find no evidence of $C P V$. The measurement of $A_{\Gamma}$ is the most precise single measurement of the parameter. The value of $y_{C P}$ is higher than our result but still statistically compatible.

If these results are going to be confirmed, the updated world averages, computed by the Heavy Flavor Averaging Group [32] are shown in Fig. 7.3.

The precision on both of these observables is improved relative to previous measurements. The precision obtained is $0.16 \%$. 

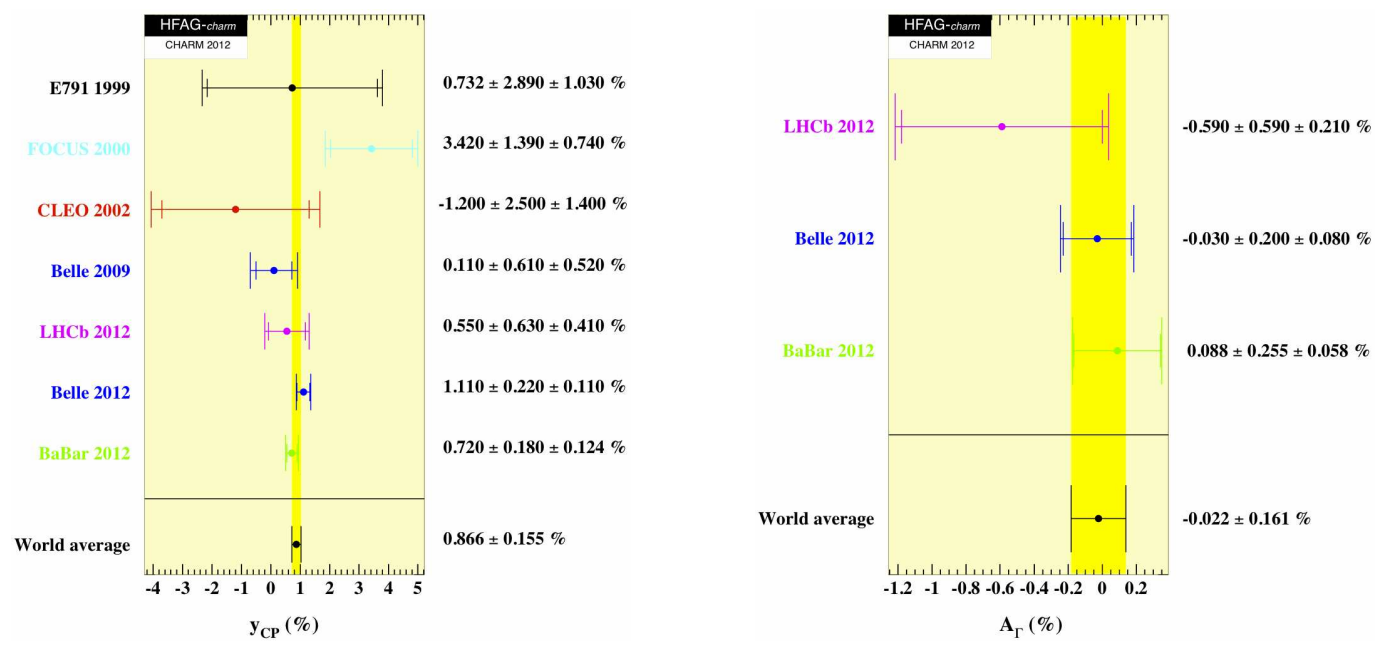

Figure 7.3: World average of the $y_{C P}$ (left) and $A_{\Gamma}$ (right) measurements including the new Belle preliminary results, computed by HFAG [32].

\subsection{Future Perspectives}

The $y_{C P}$ and $\Delta Y$ measurements are important measurements in the charm sector since they constrain the mixing parameters $x_{\mathrm{D}}$ and $y_{\mathrm{D}}$ and the $C P V$ parameter $\phi$. Although there are difficulties in the interpretation of the results due to significant theoretical uncertainties, improving the precision of the measurements of these observables can lead to insight in physics beyond the SM.

All measurements of $y_{C P}$ and $\Delta Y$ are statistically limited, as well as most of the mixing and $C P V$ measurements. The lifetime ratio analysis benefits from the fact that, at first order, the systematics affecting the lifetime measurements cancel in the ratio. The cancellation of the systematic effects is even more evident for $\Delta Y$ since there is also a difference at the numerator. To improve the precision of these measurement it is necessary to increase the statistics of the samples.

For what concerns $B A B A R$, the measurement presented in this thesis is the final measurement of $y_{C P}$ and $\Delta Y$. The measurement of $y_{C P}$ is the most precise single measurement.

The other B-Factory experiment, Belle, can count on an integrated luminosity that is around a factor two of the BABAR one. The Belle Collaboration has shown preliminary measurements of $y_{C P}$ and $\Delta Y$ using the tagged-channels only, therefore, if the results are confirmed, we do no expect an improvement on $\Delta Y$ from their data. Their measurement of $\Delta Y$ is the most precise single measurement and the one of $y_{C P}$ shows the highest significance for mixing, $4.5 \sigma$. The measurement of $y_{C P}$, on the other hand, can be improved adding the untagged channels and increasing significantly the statistical precision.

The LHCb Collaboration has presented a measurement in 2012 using 2010 data, corresponding to only $29 \mathrm{pb}^{-1}$ at $\mathrm{CM}$ energy of $7 \mathrm{TeV}$, the errors are roughly a factor 3 worst than the Belle and BABAR ones. From the point of view of the statistics, this experiment 
is increasing the data sample and should have now a sample of $D^{0}$ and $\bar{D}^{0}$ decays comparable to the one of the B-factories. The measurement should be updated to the full available data sample and it would be very interesting to see their central value of $y_{C P}$, that can discriminate between the higher Belle and the lower BABAR value. Of course, the measurement of $\Delta Y$ ( or $A_{\Gamma}$ ) is also very interesting, although it seems that with the current statistics there is no sensitivity to $C P V$. The delay in this update and the fact that the systematic errors for the LHCb measurement are not significantly lower the the statistical, as for the B-Factories, may indicate that these high precision measurements are still hard to do at proton-proton machine.

The future SuperB-Factories are ideal places for these time-dependent high precision measurements. The existing SuperB and Belle II projects of the machine and the detector promise to reach luminosities of $8 \times 10^{35} \mathrm{~cm}^{-2} \mathrm{~s}^{-1}$ (Belle II) and $10^{36} \mathrm{~cm}^{-2} \mathrm{~s}^{-1}$ (SuperB) and maintain the vertex resolutions as in Belle/BABAR. With a factor 100 of integrated luminosities the $y_{C P}$ and $\Delta Y$ measurement will reach the limit of the systematic error but should be enough precise to determine whether $y_{\mathrm{D}}$ and $y_{C P}$ are compatible and could be sensitive also to the direct $C P V$. 



\section{Appendix A}

\section{The Area of the Unitary Triangles}

Let's consider a Unitary Triangle (UT), for example:

$$
V_{u d} V_{u b}^{*}+V_{c d} V_{c b}^{*}+V_{t d} V_{t b}^{*}=0 .
$$

Considering the complex plane as a vector space, a triangle is identified by two vectors, we can choose $\vec{p}_{1}=V_{u d} V_{u b}^{*}$ and $\vec{p}_{2}=V_{c d} V_{c b}^{*}$, and the area of the triangle is given by their vector product:

$$
A=\frac{1}{2}\left|\vec{p}_{2} \times \vec{p}_{1}\right|
$$

We can compute the vector product expliciting the real and imaginary parts of the vectors:

$$
\begin{aligned}
A & =\frac{1}{2}\left|\frac{p_{2}+p_{2}^{*}}{2} \frac{p_{1}-p_{1}^{*}}{2}-\frac{p_{2}-p_{2}^{*}}{2} \frac{p_{1}+p_{1}^{*}}{2}\right| \\
& =\frac{1}{4}\left|p_{2}^{*} p_{1}-p_{2} p_{1}^{*}\right| \\
& =\frac{1}{2}\left|\Im\left(p_{2}^{*} p_{1}\right)\right|,
\end{aligned}
$$

therefore:

$$
A=\frac{1}{2}\left|\Im\left(V_{u d} V_{u b}^{*} V_{c d}^{*} V_{c b}\right)\right|=\frac{1}{2} J .
$$

where the quantity $J=\left|\Im\left(V_{u d} V_{u b}^{*} V_{c d}^{*} V_{c b}\right)\right|$ is independent of the quark phases since each quark enters in two CKM elements, one of which is a complex conjugate.

The choice of $\vec{p}_{1}$ and $\vec{p}_{2}$ has been completely arbitrary, indeed any couple of vectors from Eq. (A.1) identifies the same triangle, with the same area, therefore:

$$
\left|\Im\left(V_{u d} V_{u b}^{*} V_{c d}^{*} V_{c b}\right)\right|=\left|\Im\left(V_{u d} V_{u b}^{*} V_{t d}^{*} V_{t b}\right)\right|=\left|\Im\left(V_{c d}^{*} V_{c b} V_{t d}^{*} V_{t b}\right)\right|
$$

To show that $J$ is an invariant, and therefore that all UTs have the same area, it is enough 
to repeat the same procedure for all the other UTs. Let's consider, for example, the UT triangle:

$$
V_{u d} V_{c d}^{*}+V_{u s} V_{c s}^{*}+V_{u b} V_{c b}^{*}=0 .
$$

Repeating the procedure we find:

$$
\left|\Im\left(V_{u d} V_{c d}^{*} V_{u s}^{*} V_{c s}\right)\right|=\left|\Im\left(V_{u d} V_{c d}^{*} V_{u b}^{*} V_{c b}\right)\right|=\left|\Im\left(V_{u s}^{*} V_{c s} V_{u b}^{*} V_{c b}\right)\right| .
$$

Comparing Eq. (A.5) with Eq. (A.7) we notice that first term of the former is the same as the second term of the latter. Proceeding like this for the other unitary relations, we demonstrate that all UT have the same area.

The invariant $J$ is more generally defined as:

$$
J=\left|\Im\left(V_{i j} V_{i l}^{*} V_{k l} V_{l j}^{*}\right)\right|,
$$

no sum on repeated indices, and its experimental value is $J=\left(2.91_{-0.11}^{+0.19}\right) 10^{-5}[23]$. 


\section{Appendix B}

\section{Comparison Data - MC}

In the following we report the reconstructed $D^{0}$ mass distributions for data and $\mathrm{MC}$ for the seven modes. In the tagged channels a cut $0.1447 \mathrm{GeV} / c^{2}<\Delta m<0.1463 \mathrm{GeV} / c^{2}$ has been applied.
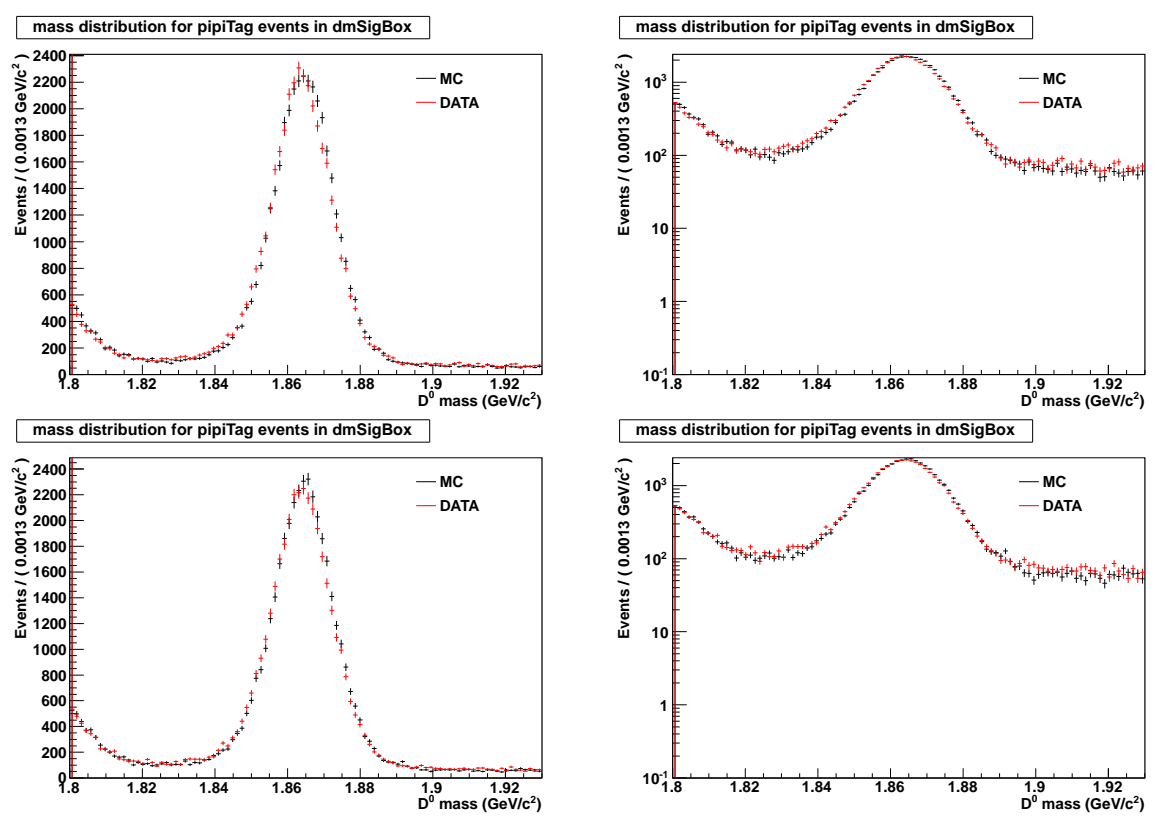

Figure B.1: Mass distribution for data (red) and MC (black) in linear (left) and logarithmic scale (right). Top the tagged $D^{0} \rightarrow \pi^{+} \pi^{-}$events, bottom the tagged $\bar{D}^{0} \rightarrow \pi^{+} \pi^{-}$ events. The MC distribution is scaled to data luminosity. The cut $0.1447 \mathrm{GeV} / c^{2}<\Delta m<$ $0.1463 \mathrm{GeV} / c^{2}$ has been applied. 

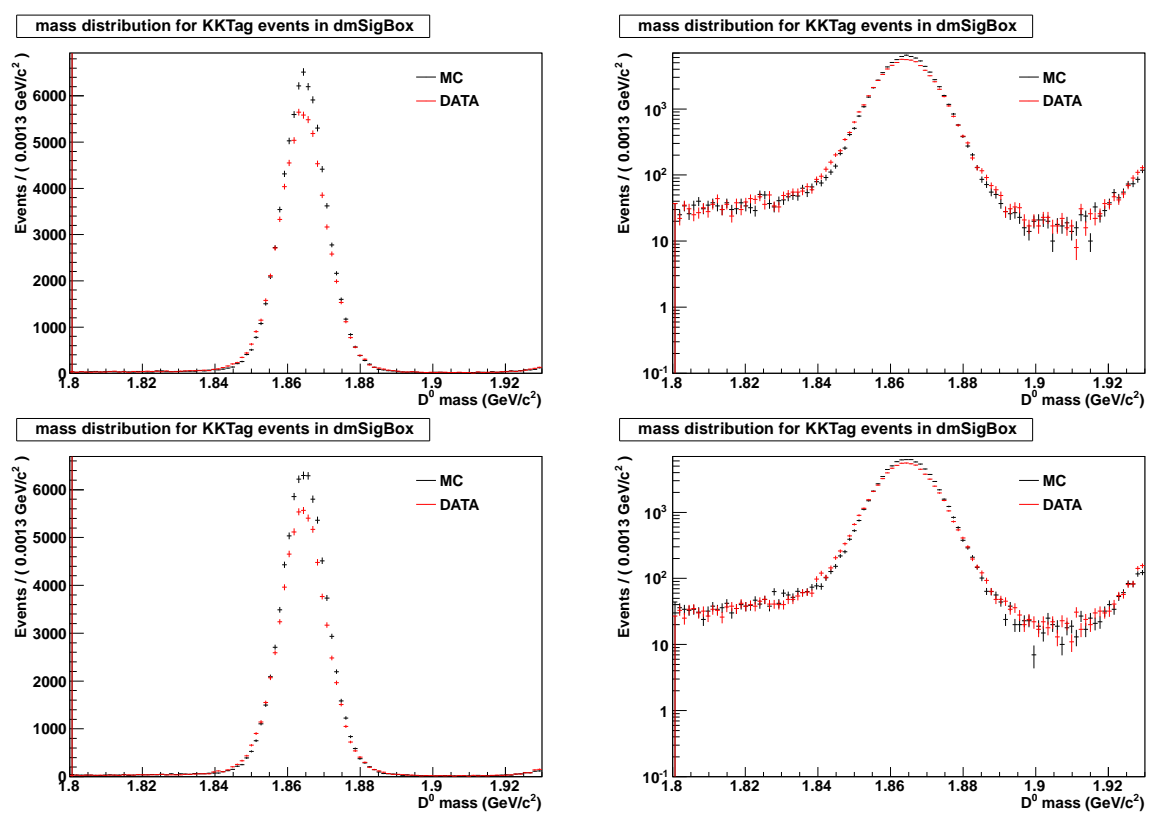

Figure B.2: Mass distribution for data (red) and MC (black) in linear (left) and logarithmic scale (right). Top the tagged $D^{0} \rightarrow K^{+} K^{-}$events, bottom the tagged $\bar{D}^{0} \rightarrow K^{+} K^{-}$ events. The MC distribution is scaled to data luminosity. The cut $0.1447 \mathrm{GeV} / c^{2}<\Delta m<$ $0.1463 \mathrm{GeV} / c^{2}$ has been applied.
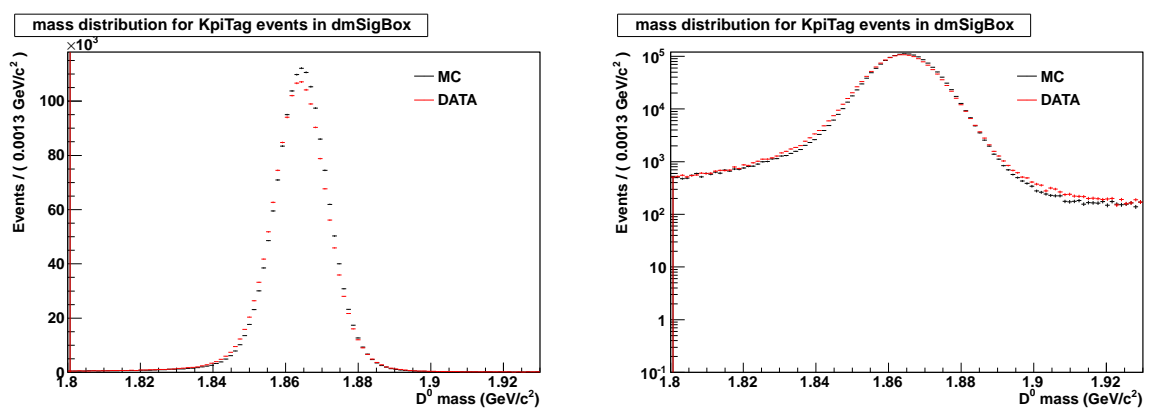

Figure B.3: Mass distribution for data (red) and MC (black) in linear (left) and logarithmic scale (right), of the tagged $K^{ \pm} \pi^{\mp}$ events. The MC distribution is scaled to data luminosity. The cut $0.1447 \mathrm{GeV} / c^{2}<\Delta m<0.1463 \mathrm{GeV} / c^{2}$ has been applied.
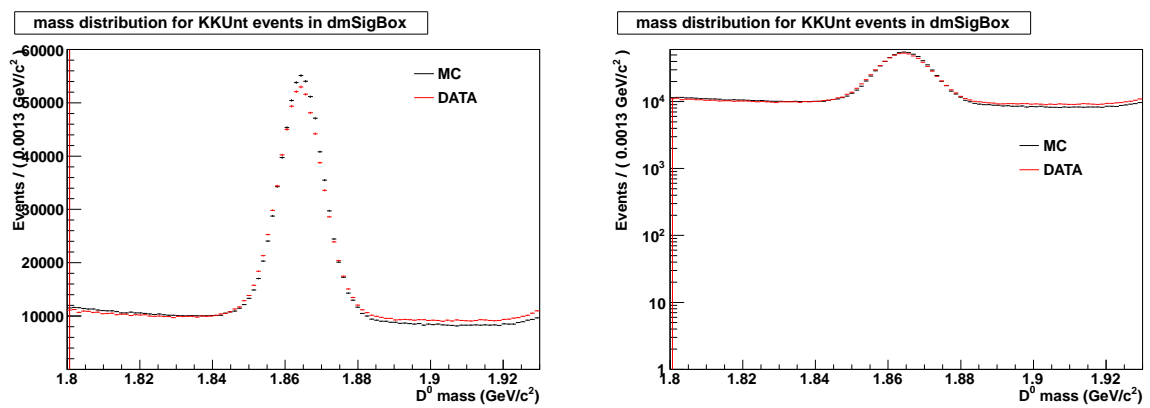

Figure B.4: Mass distribution for data (red) and MC (black) in linear (left) and logarithmic scale (right), of the untagged $K^{+} K^{-}$events. The MC distribution is scaled to data luminosity. 

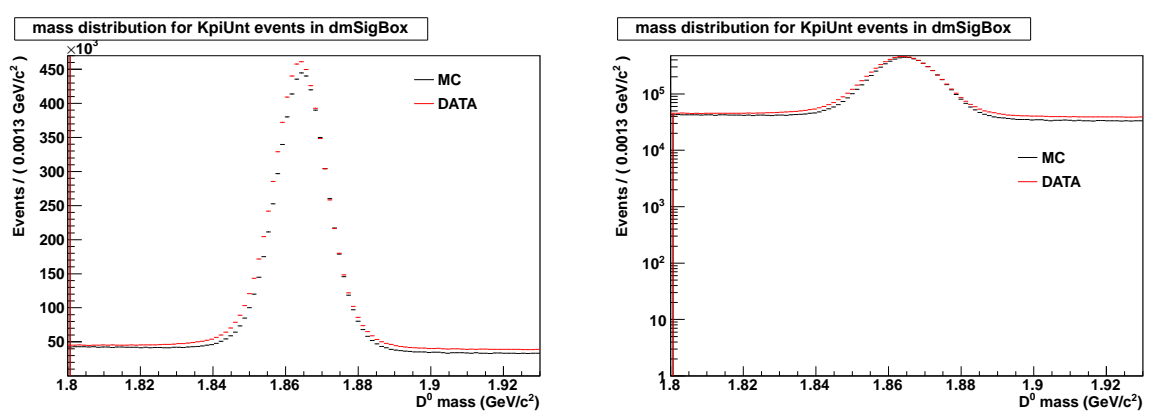

Figure B.5: Mass distribution for data (red) and MC (black) in linear (left) and logarithmic scale (right), of the untagged $K^{ \pm} \pi^{\mp}$ events. The MC distribution is scaled to data luminosity. 


\section{Appendix C}

\section{Mass Fits Parameters}

In the following we report the explicit forms of the PDFs and the extracted value of the parameters from the data mass fits of each of the five channels, described in section 4.3.1.

\section{Tagged $\pi^{+} \pi^{-}$Channel}

We fit the $D^{0}$ and $\bar{D}^{0}$ decays simultaneously, all the parameters are shared except the expected number of events in each category that are depending on the flavor: $N_{\text {sig }}=$ $\left\{N_{\text {sig }}^{D^{0}}, N_{\text {sig }}^{\bar{D}^{0}}\right\}$, and $N_{\text {bkg }}^{D^{0}}, N_{\text {bkg }}^{\bar{D}^{0}}$. The signal PDF $\mathcal{S}$ and the background PDF $\mathcal{B}$ are:

$$
\begin{aligned}
& \mathcal{S}(m)=N_{\text {sig }}\left\{f_{1} G\left(m ; \mu_{1}, \sigma_{1}\right)+\left(1-f_{1}\right)\left[f_{21} G\left(m ; \mu_{2}, \sigma_{2}\right)\right]\right\} \\
& \mathcal{B}(m)=N_{\text {bkg }}\left\{C\left[1+c_{1} m\right]\right\},
\end{aligned}
$$

where the parameter $C$ is the normalization over the fit range. The extracted values of the parameters are reported in Tab. C.1.

\begin{aligned} & \hline \hline signal \\ & \hline$N_{\mathrm{sig}}^{D^{0}}=34101 \pm 234 \\ & N_{\mathrm{sig}}=34152 \pm 234 \\ & f_{1}=0.33 \pm 0.08 \\ & \mu_{1}=(1861.8 \pm 0.5) \mathrm{MeV} / c^{2} \\ & \sigma_{1}=(11.4 \pm 0.7) \mathrm{MeV} / c^{2} \\ & \mu_{2}=(1.8642 \pm 0.1) \mathrm{MeV} / c^{2} \\ & \sigma_{2}=(7.3 \pm 0.2) \mathrm{MeV} / c^{2} \\ &$\hline \hline\end{aligned}

\begin{aligned} & \hline \hline background \\ & \hline$N_{\mathrm{bkg}}^{D^{0}}=7471 \pm 168 \\ & N_{\mathrm{bkg}}^{\bar{D}^{0}}=7486 \pm 168 \\ & c_{1}=(-0.291 \pm 0.02) /\left(\mathrm{GeV} / c^{2}\right) \\ &$\hline \hline\end{aligned}

Table C.1: Parameter values and errors from the simultaneous fit to the reconstructed $D^{0}$ and $\bar{D}^{0}$ mass distributions of the tagged $\pi^{+} \pi^{-}$events. The correspondent plots are in Fig. 4.2. 


\section{Tagged $K^{+} K^{-}$Channel}

We fit the $D^{0}$ and $\bar{D}^{0}$ decays simultaneously, all the parameters are shared except the expected number of events in each category that are depending on the flavor: $N_{\text {sig }}=$ $\left\{N_{\text {sig }}^{D^{0}}, N_{\text {sig }}^{\bar{D}^{0}}\right\}$, and $N_{\text {bkg }}^{D^{0}}, N_{\text {bkg }}^{\bar{D}^{0}}$. The signal PDF $\mathcal{S}$ and the background PDF $\mathcal{B}$ are:

$$
\begin{aligned}
\mathcal{S}(m)= & N_{\text {sig }}\left\{f_{C B} C B\left(m ; m_{0}, \sigma_{C B}, \alpha, n\right)\right. \\
& \left(1-f_{C B}\right)\left[f_{1} G\left(m ; \mu_{1}, \sigma_{1}\right)+\right. \\
& \left.\left.\left(1-f_{1}\right) G\left(m ; \mu_{2}, \sigma_{2}\right)\right]\right\}, \\
\mathcal{B}(m)= & N_{\text {bkg }}\left\{C\left[1+c_{1} m\right]\right\},
\end{aligned}
$$

where the parameter $C$ is the normalization over the fit range. The extracted values of the parameters are reported in Tab. C.2.

\begin{aligned} & \hline \hline \multicolumn{1}{c}{ signal } \\ & \hline$N_{\mathrm{sig}}^{D^{0}}=70271 \pm 289 \\ & N_{\mathrm{sig}}^{D^{0}}=69532 \pm 287 \\ & f_{1}=0.05 \pm 0.04 \\ & \mu_{1}=(1864.3 \pm 0.3) \mathrm{MeV} / c^{2} \\ & \sigma_{2}=(6.2 \pm 0.2) \mathrm{MeV} / c^{2} \\ & \mu_{2}=(1864.28 \pm 0.04) \mathrm{MeV} / c^{2} \\ & \sigma_{1}=(3.4 \pm 0.9) \mathrm{MeV} / c^{2} \\ & f_{C B}=(0.17 \pm 0.04) \\ & m_{0}=(1863.4 \pm 0.2) \mathrm{MeV} / c^{2} \\ & \sigma_{C B}=(10.7 \pm 0.6) \mathrm{MeV} / c^{2} \\ & \alpha=(2.3 \pm 0.2) \\ & n=3$ fixed \\ & \hline \hline\end{aligned}

\begin{aligned} & \hline \hline background \\ & \hline$N_{\mathrm{bkg}}^{D^{0}}=1874 \pm 124 \\ & N_{\mathrm{bkg}}^{\bar{D}^{0}}=1910 \pm 123 \\ & c_{1}=(-0.378 \pm 0.05) /\left(\mathrm{GeV} / c^{2}\right) \\ &$\hline \hline\end{aligned}

Table C.2: Parameter values and errors from the simultaneous fit to the reconstructed $D^{0}$ and $\bar{D}^{0}$ mass distributions of the tagged $K^{+} K^{-}$events. The correspondent plots are in Fig. 4.2.

\section{Tagged $K^{ \pm} \pi^{\mp}$ Channel}

We fit the $D^{0}$ and $\bar{D}^{0}$ decays together, not distinguishing the $D$ flavor. The signal PDF $\mathcal{S}$ and the background PDF $\mathcal{B}$ are:

$$
\begin{aligned}
\mathcal{S}(m)= & N_{\mathrm{sig}}\left\{f_{1} G\left(m ; \mu_{1}, \sigma_{1}\right)+\left(1-f_{1}\right)\left[f_{21} G\left(m ; \mu_{2}, \sigma_{2}\right)+\right.\right. \\
& \left.\left.\left(1-f_{21}\right) G\left(m ; \mu_{3}, \sigma_{3}\right)\right]\right\}, \\
\mathcal{B}(m)= & N_{\text {bkg }}\left\{C\left[1+c_{1} m+c_{2}\left(2 m^{2}-1\right)\right]\right\},
\end{aligned}
$$


where the parameter $C$ is the normalization over the fit range. The extracted values of the parameters are reported in Tab. C.3.

\begin{aligned} & \hline \hline \multicolumn{1}{c}{ signal } \\ & \hline$N_{\text {sig }}=1567062 \pm 2040 \\ & f_{1}=0.43 \pm 0.03 \\ & \mu_{1}=(1863.71 \pm 0.05) \mathrm{MeV} / c^{2} \\ & \sigma_{1}=(9.2 \pm 0.2) \mathrm{MeV} / c^{2} \\ & f_{21}=0.901 \pm 0.010 \\ & \mu_{2}=(1864.15 \pm 0.02) \mathrm{MeV} / c^{2} \\ & \sigma_{2}=(6.26 \pm 0.07) \mathrm{MeV} / c^{2} \\ & \mu_{3}=(1856.1 \pm 0.4) \mathrm{MeV} / c^{2} \\ & \sigma_{3}=(19.1 \pm 0.4) \mathrm{MeV} / c^{2} \\ &$\hline \hline\end{aligned}

\begin{aligned} & \hline \hline background \\ & \hline$N_{\mathrm{bkg}}=30307 \pm 1620 \\ & c_{1}=(-0.534 \pm 0.02) /\left(\mathrm{GeV} / c^{2}\right) \\ & c_{2}=(0.07 \pm 0.04) /\left(\mathrm{GeV} / c^{2}\right)^{2} \\ &$\hline \hline\end{aligned}

Table C.3: Parameter values and errors from the fit to the reconstructed $D$ mass distribution of the tagged $K^{ \pm} \pi^{\mp}$ events. The correspondent plot is in Fig. 4.2.

\section{Untagged $K^{+} K^{-}$Channel}

The signal PDF $\mathcal{S}$ and the background PDF $\mathcal{B}$ are:

$$
\begin{aligned}
\mathcal{S}(m)= & N_{\text {sig }}\left\{f_{1} G\left(m ; \mu_{1}, \sigma_{1}\right)+\left(1-f_{1}\right)\left[f_{21} G\left(m ; \mu_{2}, \sigma_{2}\right)+\right.\right. \\
& \left.\left.\left(1-f_{21}\right) G\left(m ; \mu_{3}, \sigma_{3}\right)\right]\right\}, \\
\mathcal{B}(m)= & N_{\text {bkg }}\left\{C\left[1+c_{1} m+c_{2}\left(2 m^{2}-1\right)\right]\right\},
\end{aligned}
$$

where the parameter $C$ is the normalization over the fit range. The extracted values of the parameters are reported in Tab. C.4.

\begin{aligned} & \hline \hline \multicolumn{1}{c}{ signal } \\ & \hline$N_{\text {sig }}=530209 \pm 2514 \\ & f_{1}=0.36 \pm 0.10 \\ & \mu_{1}=(1864.21 \pm 0.05) \mathrm{MeV} / c^{2} \\ & \sigma_{1}=(5.0 \pm 0.3) \mathrm{MeV} / c^{2} \\ & f_{21}=0.997 \pm 0.001 \\ & \mu_{2}=(1864.22 \pm 0.05) \mathrm{MeV} / c^{2} \\ & \sigma_{2}=(7.5 \pm 0.3) \mathrm{MeV} / c^{2} \\ & \mu_{3}=(1844 \pm 1) \mathrm{MeV} / c^{2} \\ & \sigma_{3}=(3 \pm 1) \mathrm{MeV} / c^{2} \\ &$\hline \hline\end{aligned}

\begin{aligned} \hline \hline \multicolumn{1}{c}{ background } \\ \hline$N_{\mathrm{bkg}}=663236 \pm 2535 \\ c_{1}=(-0.0478 \pm 0.002) /\left(\mathrm{GeV} / c^{2}\right) \\ c_{2}=(-0.0010 \pm 0.005) /\left(\mathrm{GeV} / c^{2}\right)^{2} \\$\hline\end{aligned}

Table C.4: Parameter values and errors from the fit to the reconstructed mass distribution of the untagged $K^{+} K^{-}$events. The correspondent plot is in Fig. 4.3. 


\section{Untagged $K^{ \pm} \pi^{\mp}$ Channel}

The signal PDF $\mathcal{S}$ and the background PDF $\mathcal{B}$ are:

$$
\begin{aligned}
\mathcal{S}(m)= & N_{\mathrm{sig}}\left\{f_{1} G\left(m ; \mu_{1}, \sigma_{1}\right)+\left(1-f_{1}\right)\left[f_{21} G\left(m ; \mu_{2}, \sigma_{2}\right)+\right.\right. \\
& \left.\left.\left(1-f_{21}\right) G\left(m ; \mu_{3}, \sigma_{3}\right)\right]\right\}, \\
\mathcal{B}(m)= & N_{\mathrm{bkg}}\left\{C\left[1+c_{1} m+c_{2}\left(2 m^{2}-1\right)\right]\right\},
\end{aligned}
$$

where the parameter $C$ is the normalization over the fit range. The extracted values of the parameters are reported in Tab. C.4.

\begin{aligned} & \hline \hline \multicolumn{1}{c}{ signal } \\ & \hline$N_{\mathrm{sig}}=6157417 \pm 14084 \\ & f_{1}=0.36 \pm 0.01 \\ & \mu_{1}=(1864.22 \pm 0.02) \mathrm{MeV} / c^{2} \\ & \sigma_{1}=(5.88 \pm 0.03) \mathrm{MeV} / c^{2} \\ & f_{21}=0.856 \pm 0.004 \\ & \mu_{2}=(1863.79 \pm 0.03) \mathrm{MeV} / c^{2} \\ & \sigma_{2}=(8.36 \pm 0.05) \mathrm{MeV} / c^{2} \\ & \mu_{3}=(1859.7 \pm 0.2) \mathrm{MeV} / c^{2} \\ & \sigma_{3}=(17.4 \pm 0.4) \mathrm{MeV} / c^{2} \\ &$\hline \hline\end{aligned}

\begin{tabular}{rcc}
\hline \hline & background \\
\hline$N_{\text {bkg }}=$ & $3469852 \pm 13910$ \\
$c_{1}$ & $=(-0.0743 \pm 0.002) /\left(\mathrm{GeV} / c^{2}\right)$ \\
$c_{2}$ & $=(0.021 \pm 0.003) /\left(\mathrm{GeV} / c^{2}\right)^{2}$ \\
\hline \hline
\end{tabular}

Table C.5: Parameter values and errors from the fit to the reconstructed mass distribution of the untagged $K^{ \pm} \pi^{\mp}$ events. The correspondent plot is in Fig. 4.3. 


\section{Appendix D}

\section{Signal Region Optimization Studies}

\section{D.1 Asymmetry as a Function of the Signal Region Width}

Given the mass PDFs extracted from the fits described in Sec. 4.3.1, we study the asymmetry $\alpha$ as a function of the signal region width $w$, imposing the constrain that the number of events contained in the right side of the interval is the same as the number of events contained in the left side.

In Fig. D.1 we report the value of the asymmetry as a function of the width. The asym-
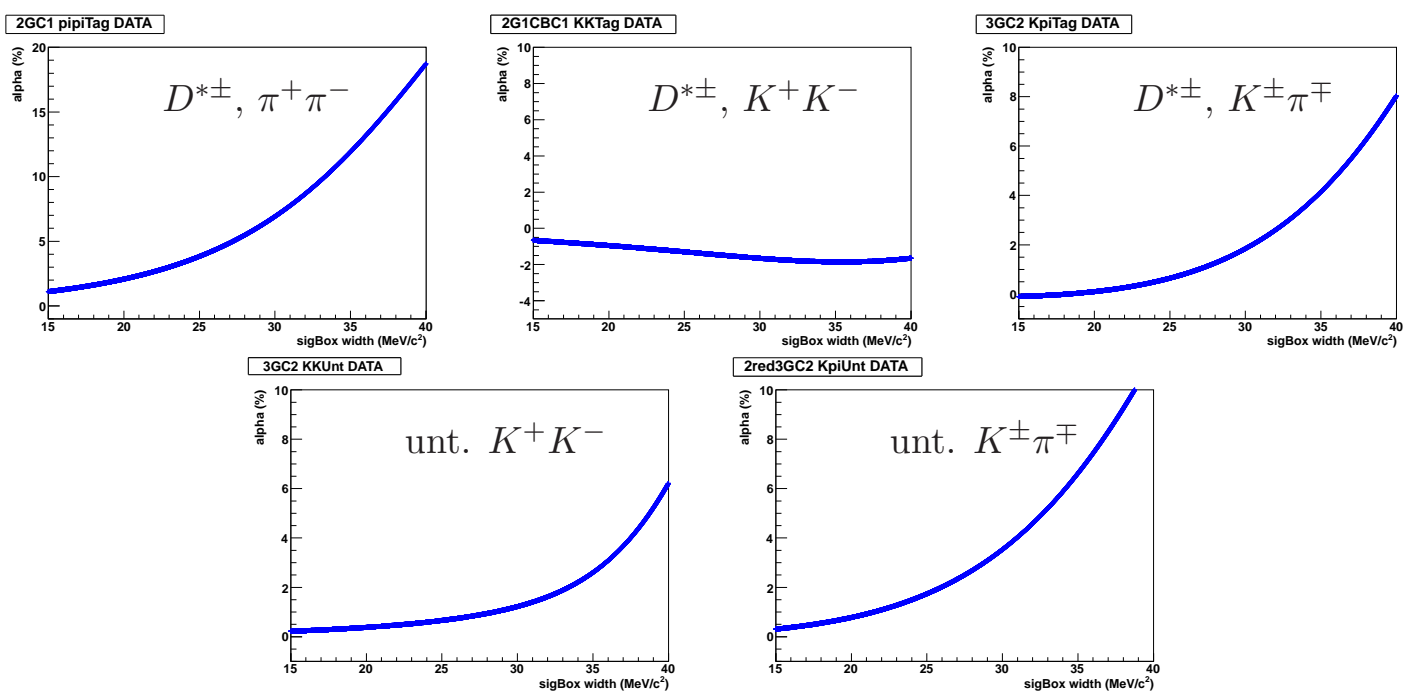

Figure D.1: Asymmetry as a function of the width of the signal region for data.

metry is smaller than $\sim 7 \%$ for all the channels, except the tagged $\pi^{+} \pi^{-}$, in which it is slightly above $10 \%$. The tagged $\pi^{+} \pi^{-}$channel is the lowest statistics one. In addition it is the one with the lowest value for the peak position, therefore the asymmetry is enhanced by the fact that the center of the signal region is more shifted from $m_{C}$ than the other 
channels. The value of $\alpha$ is set to zero for all the channels.

The same study on MC yields the same conclusion.

\section{D.2 Width Optimization}

\section{Lifetimes Statistical Error}

We perform Toy MC studies generating events from the proper time PDFs. For each signal region width we fix the number of signal and background events according to the integral of the mass PDFs, and we evaluate the statistical error on the lifetimes. We repeat this procedure for the five channels separately, the results are in Fig. D.2. This study suffers
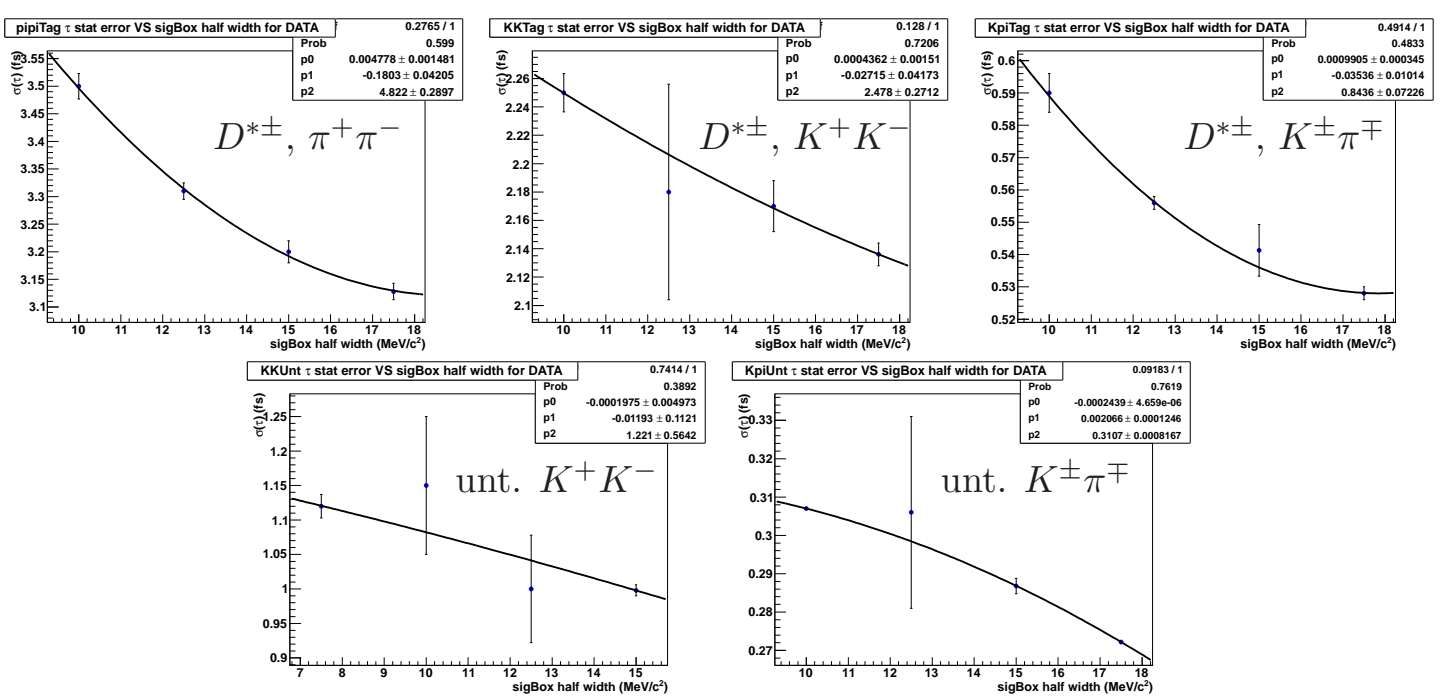

Figure D.2: Statistical error on the lifetime as a function of the half width of the signal region.

by the low statistics of the Toy samples, but suggests, as expected, to use larger signal regions for all channels (not distinguishing the tagged from the untagged).

\section{Untagged $K^{+} K^{-}$lifetime Systematic Error}

In order to evaluate the systematic impact on the untagged $K^{+} K^{-}$lifetime, we perform blind fit to $\left(t, \sigma_{t}\right)$ data distributions of the untagged $K^{+} K^{-}$channel, blinding the lifetime. The signal and background PDFs are described in Chapter 5 , for this study we use a single exponential to describe the proper time distribution of the untagged $K^{+} K^{-}$events.

We apply the systematic variations used in the previous untagged analysis:

- vary by $\pm 5 \%$ the lifetime of the misreconstructed-charm component $\left(\tau_{\mathrm{crm}}\right)$;

- vary by $\pm 5 \%$ the number of events the misreconstructed-charm component $\left(N_{\mathrm{crm}}\right)$; 
- vary by $\pm 1 \sigma$ the weighting parameter of the combinatorial PDF $\left(h_{\text {comb }}\right)$;

- vary by $\pm 1 \%$ the number of events the combinatorial component $\left(N_{\text {comb }}\right)$;

The systematic error associated to each of these variations is the deviation in the central value of the extracted lifetime, and it is reported in Tab. D.1. Note that this study was performed before finalizing the background PDFs, therefore the systematic impact on the lifetimes reported here can not be interpreted quantitatively as a systematic error but it just gives the trend of the total error as a function of the signal region width.

\begin{tabular}{l|ccc}
\hline \hline & \multicolumn{4}{|c}{ Signal region Width } \\
& $20 \mathrm{MeV} / c^{2}$ & $24 \mathrm{MeV} / c^{2}$ & $30 \mathrm{MeV} / c^{2}$ \\
\hline scale $\tau_{\text {crm }}$ by $\pm 5 \%$ & $0.33 \mathrm{fs}$ & $0.37 \mathrm{fs}$ & $0.41 \mathrm{fs}$ \\
scale $N_{\text {crm }}$ by $\pm 5 \%$ & $0.09 \mathrm{fs}$ & $0.10 \mathrm{fs}$ & $0.10 \mathrm{fs}$ \\
scale $h_{\text {comb by } \pm 1 \%}$ bs & $0.01 \mathrm{fs}$ & $0.01 \mathrm{fs}$ & $0.08 \mathrm{fs}$ \\
scale $N_{\text {comb by } \pm 1 \%}$ & $0.10 \mathrm{fs}$ & $0.09 \mathrm{fs}$ & $0.10 \mathrm{fs}$ \\
\hline stat. error & $1.02 \mathrm{fs}$ & $1.00 \mathrm{fs}$ & $0.99 \mathrm{fs}$ \\
\hline total error & $1.08 \mathrm{fs}$ & $1.07 \mathrm{fs}$ & $1.08 \mathrm{fs}$ \\
\hline \hline
\end{tabular}

Table D.1: Statistical error and systematic impact of the background on the untagged $K^{+} K^{-}$lifetime for different signal region widths obtained by blind fits to data.

For the untagged $K^{+} K^{-}$channel we have chosen the signal region width that minimizes the total error: $24 \mathrm{MeV} / c^{2}$.

The same study on $\mathrm{MC}$ yields a $20 \mathrm{MeV} / c^{2}$ wide signal region. 


\section{Appendix E}

\section{Distributions of the Events in the Three Categories}

\section{E.1 Signal Events Distributions}

We report the reconstructed-mass and the $\Delta m$ distributions, and the $2 \mathrm{~d}$ scatter plot of $\left(m_{D^{0}}, \Delta m\right)$ (only for the tagged samples) for each channel, from the signal-only bigSignal MC dataset. For the untagged $K^{ \pm} \pi^{\mp}$ channel the bigSignal sample was too big, and we have used cocktail1 to produce the plots. We also show the proper time and the proper time error distributions, and the $2 \mathrm{~d}$ scatter plot $\left(t, \sigma_{t}\right)$ of these two variables.
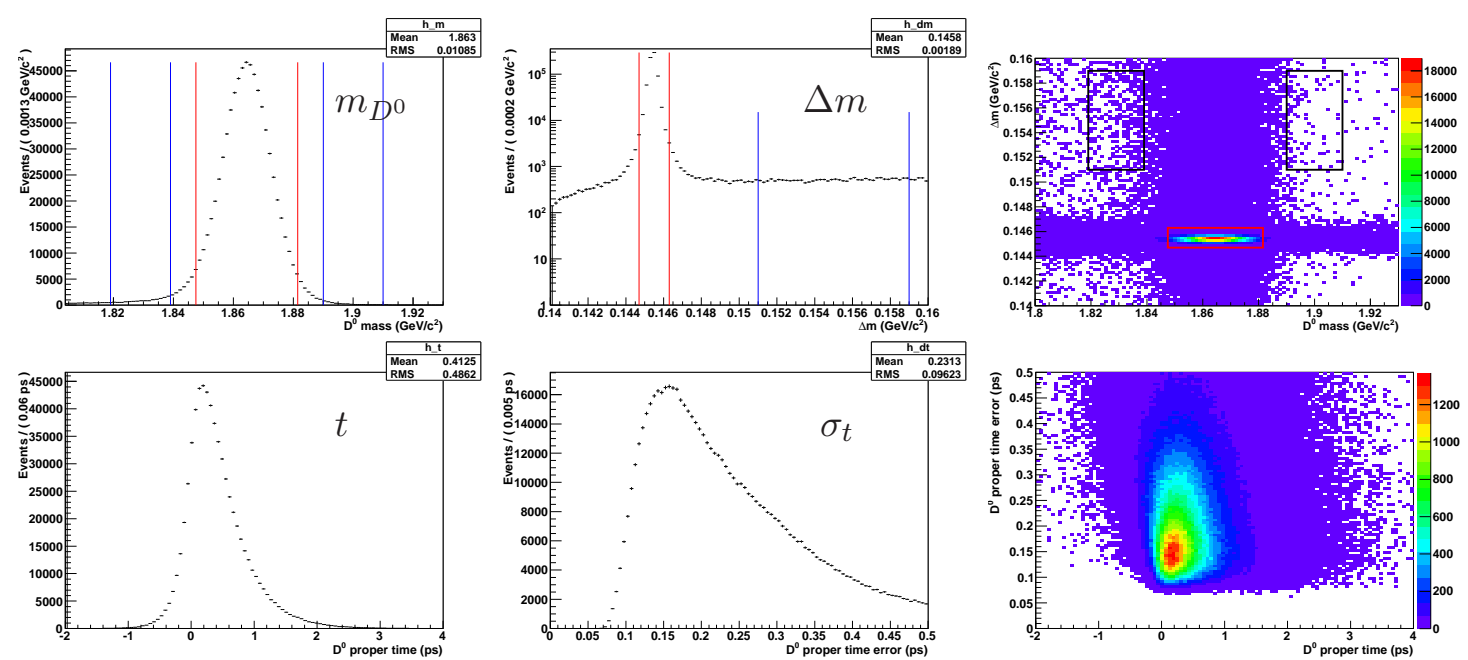

Figure E.1: Distributions of the tagged $\pi^{+} \pi^{-}$signal truth-matched events. Top row: (left) $m_{D^{0}}$, (center) $\Delta m$ distribution and (right) 2 d scatted plot $\left(m_{D^{0}}, \Delta m\right)$. The signal region is indicated by a red line, the sidebands by a blue line. Bottom row: (left) $t$, (center) $\sigma_{t}$ distributions and (right) $2 \mathrm{~d}$ scatter plot $\left(t, \sigma_{t}\right)$ for events in the signal region. 

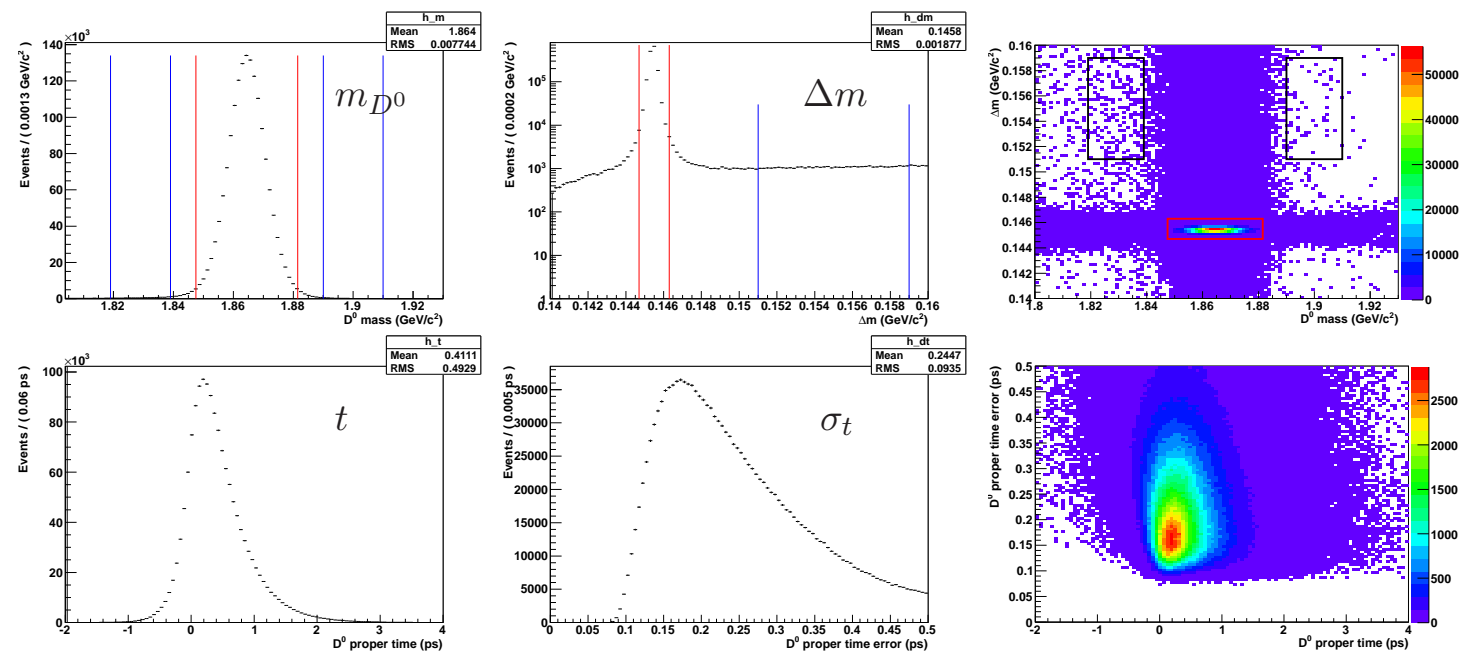

Figure E.2: Distributions of the tagged $K^{+} K^{-}$signal truth-matched events. Top row: (left) $m_{D^{0}}$, (center) $\Delta m$ distribution and (right) $2 \mathrm{~d}$ scatted plot $\left(m_{D^{0}}, \Delta m\right)$. The signal region is indicated by a red line, the sidebands by a blue line. Bottom row: (left) $t$, (center) $\sigma_{t}$ distributions and (right) $2 \mathrm{~d}$ scatter plot $\left(t, \sigma_{t}\right)$ for events in the signal region.
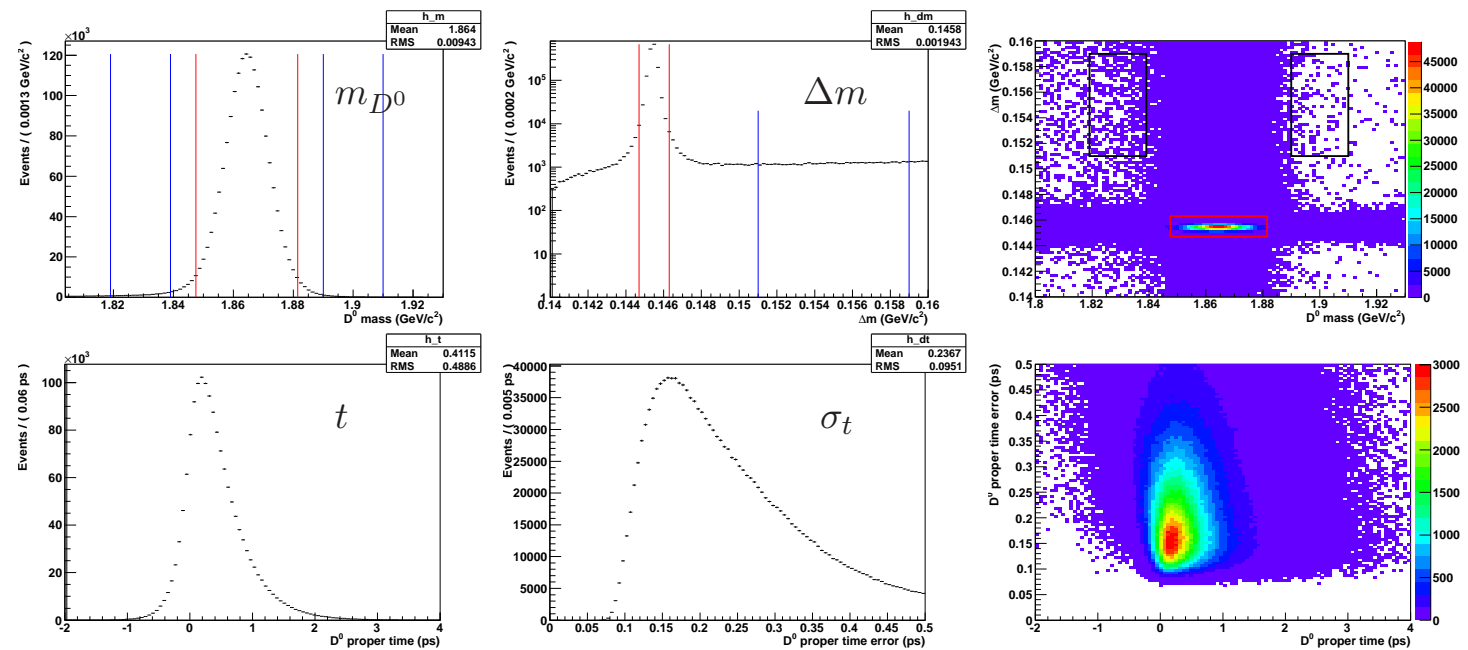

Figure E.3: Distributions of the tagged $K^{ \pm} \pi^{\mp}$ signal truth-matched events. Top row: (left) $m_{D^{0}}$, (center) $\Delta m$ distribution and (right) 2 d scatted plot $\left(m_{D^{0}}, \Delta m\right)$. The signal region is indicated by a red line, the sidebands by a blue line. Bottom row: (left) $t$, (center) $\sigma_{t}$ distributions and (right) 2 d scatter plot $\left(t, \sigma_{t}\right)$ for events in the signal region. 

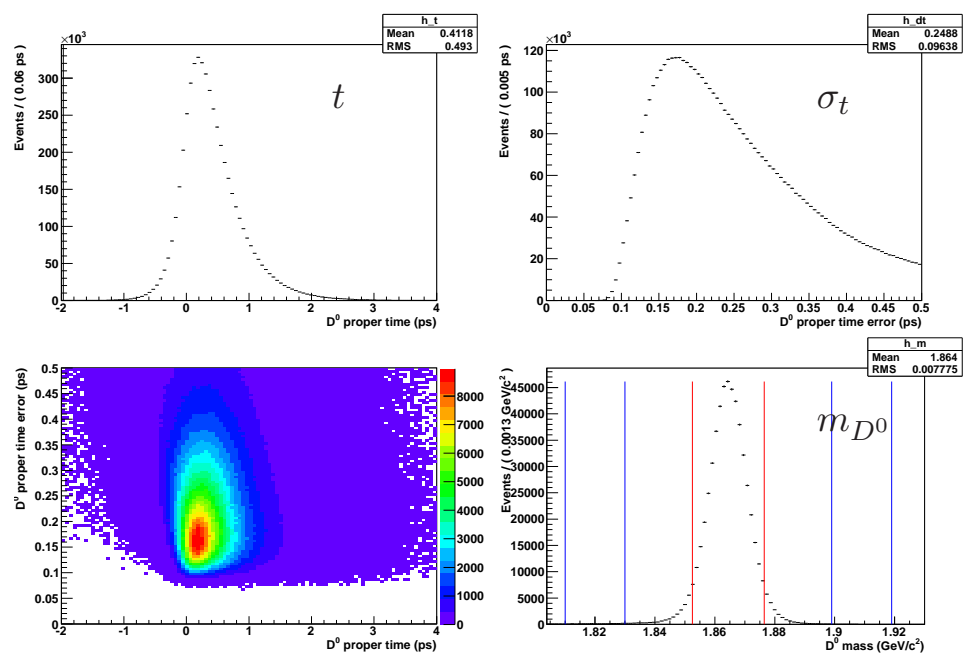

Figure E.4: Distributions of the untagged $K^{+} K^{-}$signal truth-matched events. Top row: (left) $t$ and (right) $\sigma_{t}$ distributions for events in the signal region. Bottom row: (left) $2 \mathrm{~d}$ scatter plot $\left(t, \sigma_{t}\right)$ for the events in the signal region; (right) $m_{D^{0}}$ distribution, the signal region is indicated by a red line, the sidebands by a blue line.
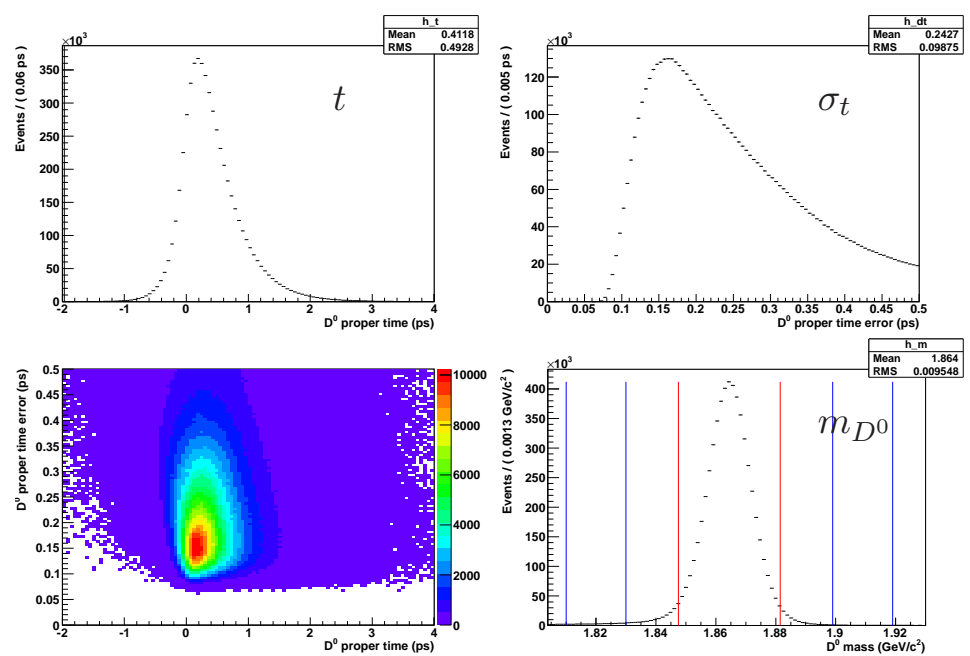

Figure E.5: Distributions of the untagged $K^{ \pm} \pi^{\mp}$ signal truth-matched events. Top row: (left) $t$ and (right) $\sigma_{t}$ distributions for events in the signal region. Bottom row: (left) $2 \mathrm{~d}$ scatter plot $\left(t, \sigma_{t}\right)$ for the events in the signal region; (right) $m_{D^{0}}$ distribution, the signal region is indicated by a red line, the sidebands by a blue line. 


\section{E.2 Misreconstructed-Charm Events Distributions}

We report the reconstructed-mass and the $\Delta m$ distributions, and the $2 \mathrm{~d}$ scatter plot of $\left(m_{D^{0}}, \Delta m\right)$ (only for the tagged samples) for each channel, from bigCharm MC dataset. We also show the proper time and the proper time error distributions, and the $2 \mathrm{~d}$ scatter plot $\left(t, \sigma_{t}\right)$ of these two variables for events in the signal region. Note that the mean of all the proper time distributions is higher than the generated $D^{0}$ lifetime, $\tau_{\text {gen }}=411.67 \mathrm{fs}$.
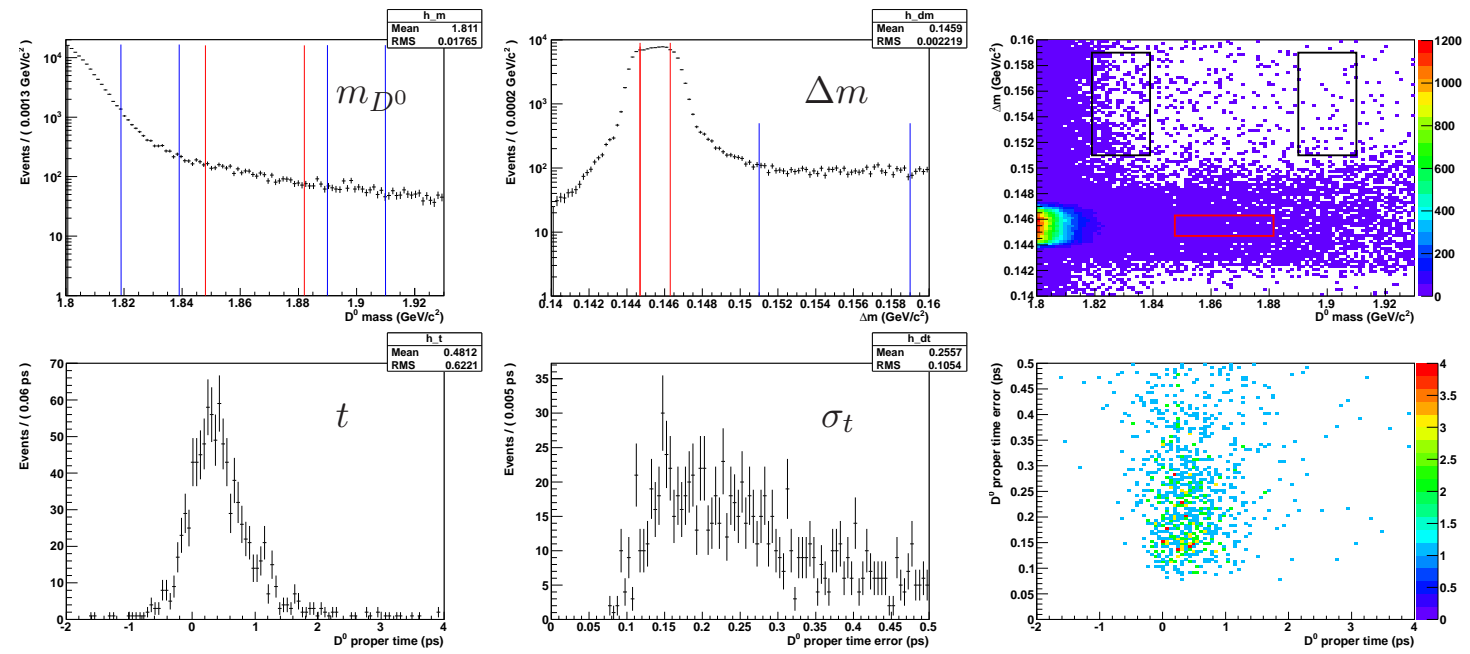

Figure E.6: Distributions of the tagged $\pi^{+} \pi^{-}$misreconstructed-charm truth-matched events. Top row: (left) $m_{D^{0}}$, (center) $\Delta m$ distribution and (right) 2 d scatted plot $\left(m_{D^{0}}, \Delta m\right)$. The signal region is indicated by a red line, the sidebands by a blue line. Bottom row: (left) $t$, (center) $\sigma_{t}$ distributions and (right) $2 \mathrm{~d}$ scatter plot $\left(t, \sigma_{t}\right)$ for events in the signal region. 

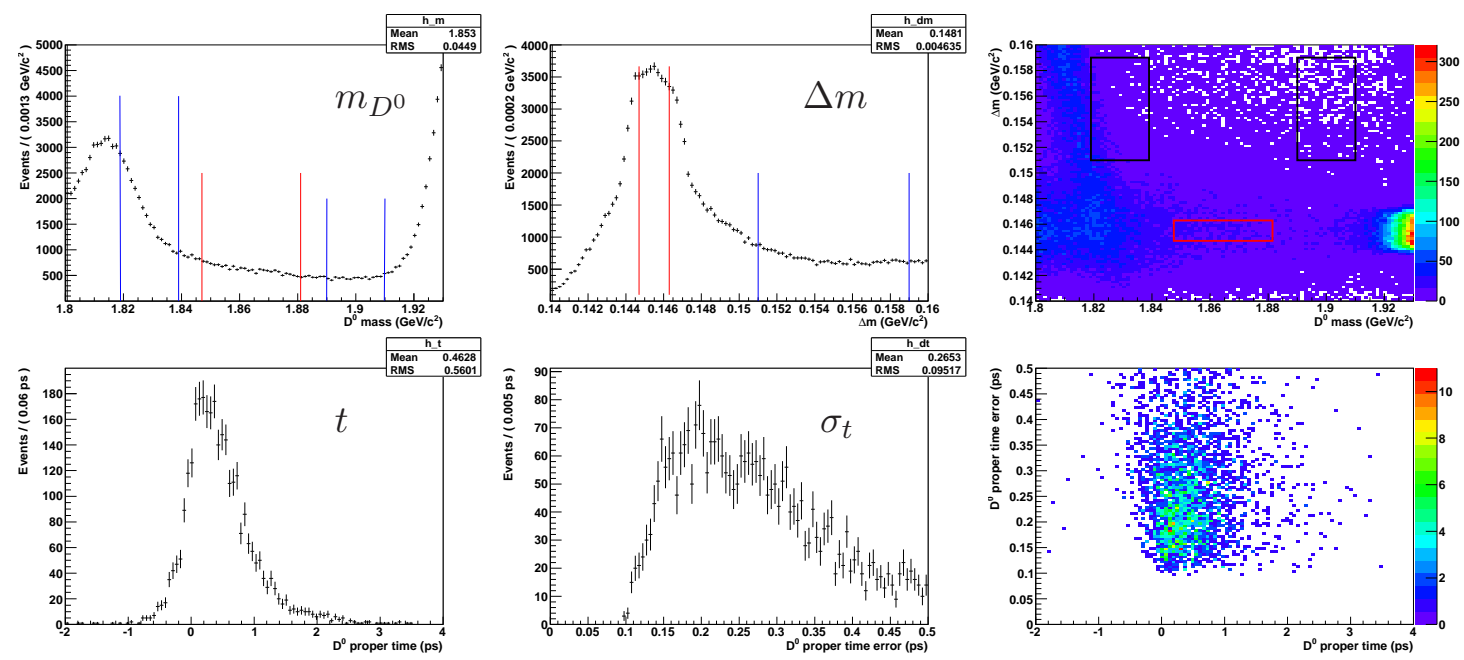

Figure E.7: Distributions of the tagged $K^{+} K^{-}$misreconstructed-charm truth-matched events. Top row: (left) $m_{D^{0}}$, (center) $\Delta m$ distribution and (right) $2 \mathrm{~d}$ scatted plot $\left(m_{D^{0}}, \Delta m\right)$. The signal region is indicated by a red line, the sidebands by a blue line. Bottom row: (left) $t$, (center) $\sigma_{t}$ distributions and (right) $2 \mathrm{~d}$ scatter plot $\left(t, \sigma_{t}\right)$ for events in the signal region.
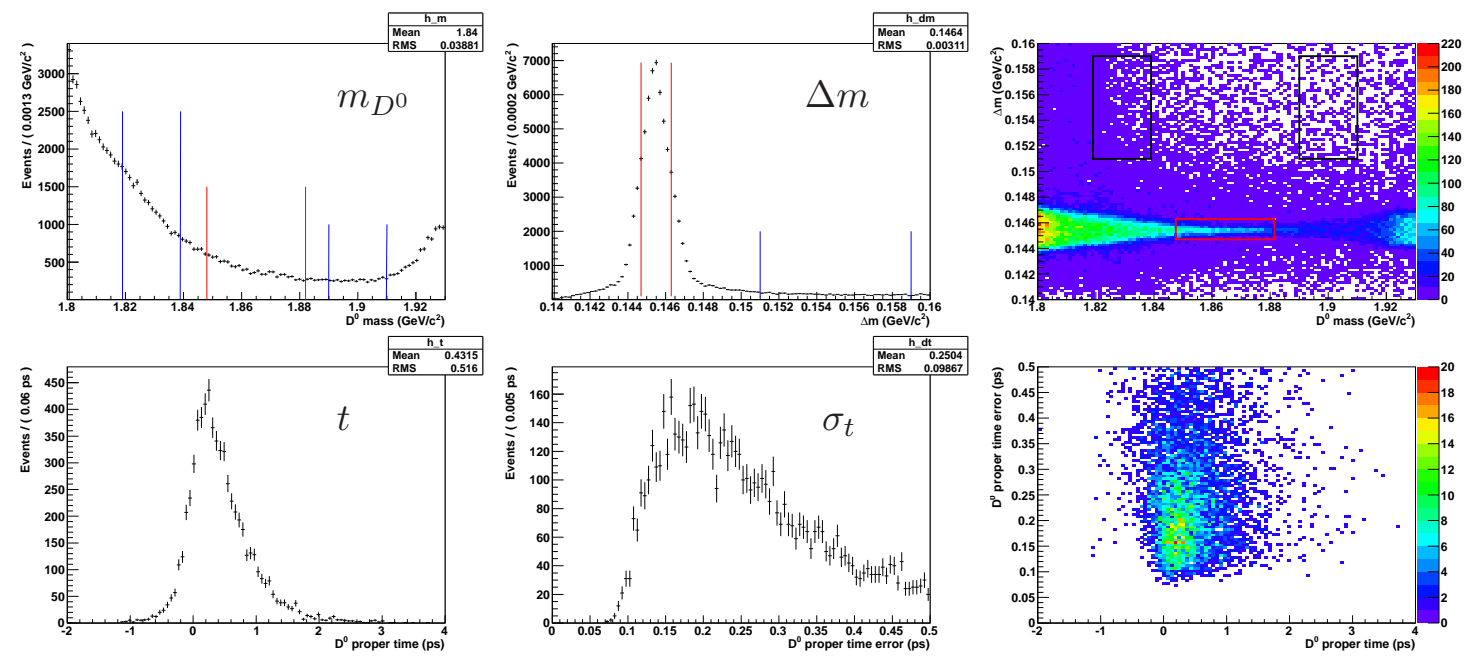

Figure E.8: Distributions of the tagged $K^{ \pm} \pi^{\mp}$ misreconstructed-charm truth-matched events. Top row: (left) $m_{D^{0}}$, (center) $\Delta m$ distribution and (right) $2 \mathrm{~d}$ scatted plot $\left(m_{D^{0}}, \Delta m\right)$. The signal region is indicated by a red line, the sidebands by a blue line. Bottom row: (left) $t$, (center) $\sigma_{t}$ distributions and (right) 2 d scatter plot $\left(t, \sigma_{t}\right)$ for events in the signal region. 

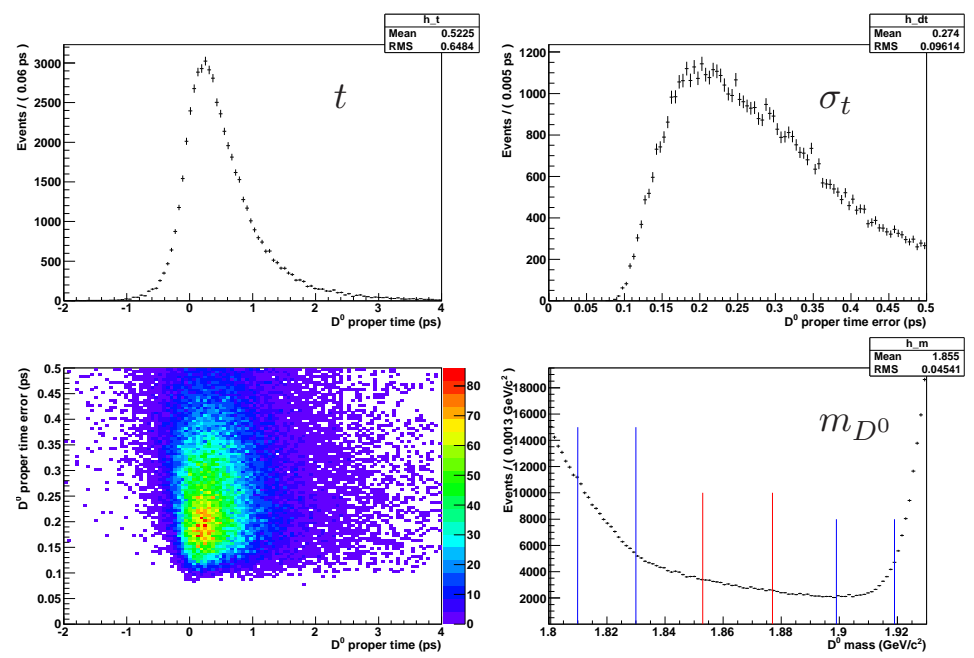

Figure E.9: Distributions of the untagged $K^{+} K^{-}$misreconstructed-charm truth-matched events. Top row: (left) $t$ and (right) $\sigma_{t}$ distributions for events in the signal region. Bottom row: (left) $2 \mathrm{~d}$ scatter plot $\left(t, \sigma_{t}\right)$ for the events in the signal region; (right) $m_{D^{0}}$ distribution, the signal region is indicated by a red line, the sidebands by a blue line.
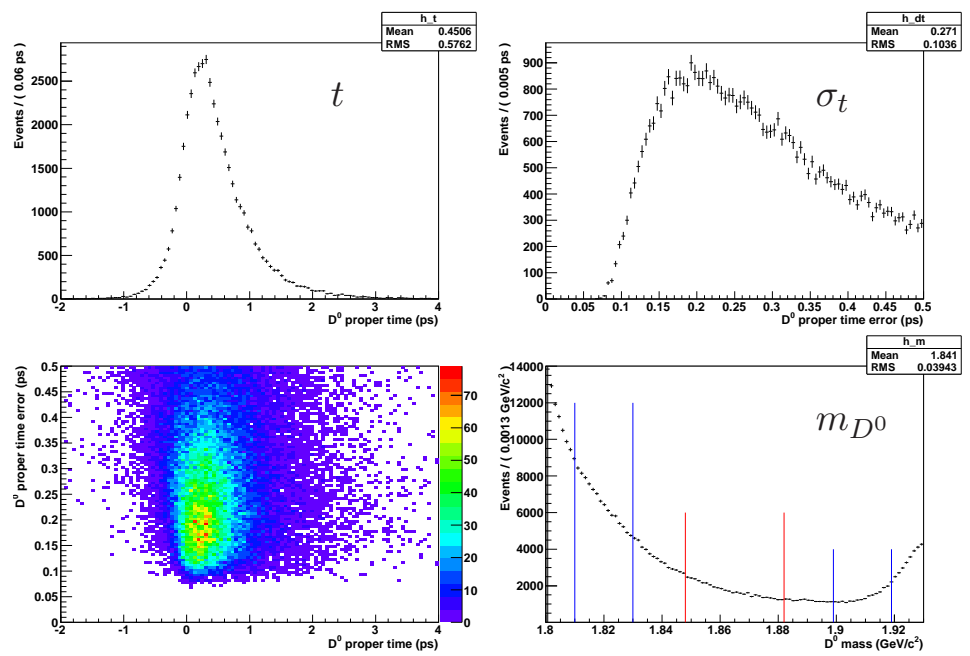

Figure E.10: Distributions of the untagged $K^{ \pm} \pi^{\mp}$ misreconstructed-charm truth-matched events. Top row: (left) $t$ and (right) $\sigma_{t}$ distributions for events in the signal region. Bottom row: (left) $2 \mathrm{~d}$ scatter plot $\left(t, \sigma_{t}\right)$ for the events in the signal region; (right) $m_{D^{0}}$ distribution, the signal region is indicated by a red line, the sidebands by a blue line. 


\section{E.3 Combinatorial Events Distributions}

We report the reconstructed-mass and the $\Delta m$ distributions, and the $2 \mathrm{~d}$ scatter plot of $\left(m_{D^{0}}, \Delta m\right)$ (only for the tagged samples) for each channel, from the bigCombin MC dataset. For the untagged $K^{ \pm} \pi^{\mp}$ channel, the bigCombin sample was too big, and we have used cocktail1 to produce the plots. We also show the proper time and the proper time error distributions, and the $2 \mathrm{~d}$ scatter plot $\left(t, \sigma_{t}\right)$ of these two variables for events in the signal region.
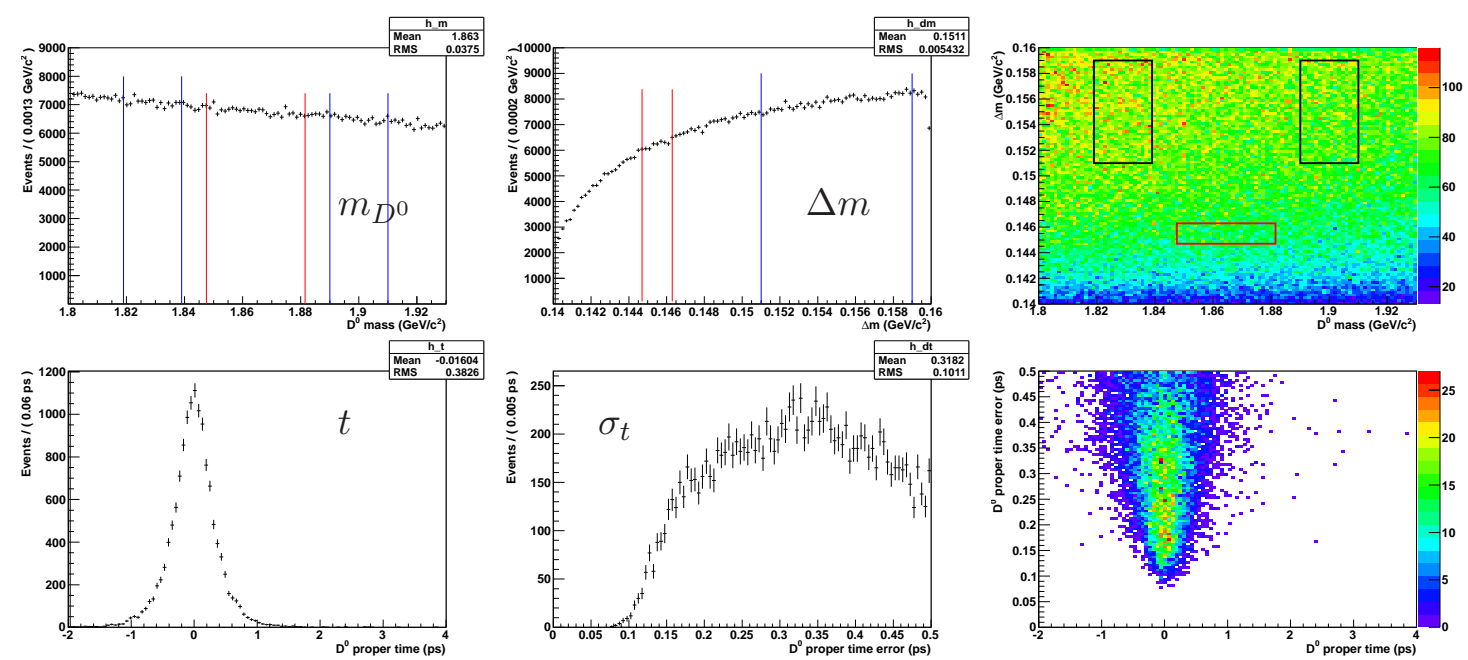

Figure E.11: Distributions of the tagged $\pi^{+} \pi^{-}$combinatorial truth-matched events. Top row: (left) $m_{D^{0}}$, (center) $\Delta m$ distribution and (right) 2 d scatted plot $\left(m_{D^{0}}, \Delta m\right)$. The signal region is indicated by a red line, the sidebands by a blue line. Bottom row: (left) $t$, (center) $\sigma_{t}$ distributions and (right) $2 \mathrm{~d}$ scatter plot $\left(t, \sigma_{t}\right)$ for events in the signal region.
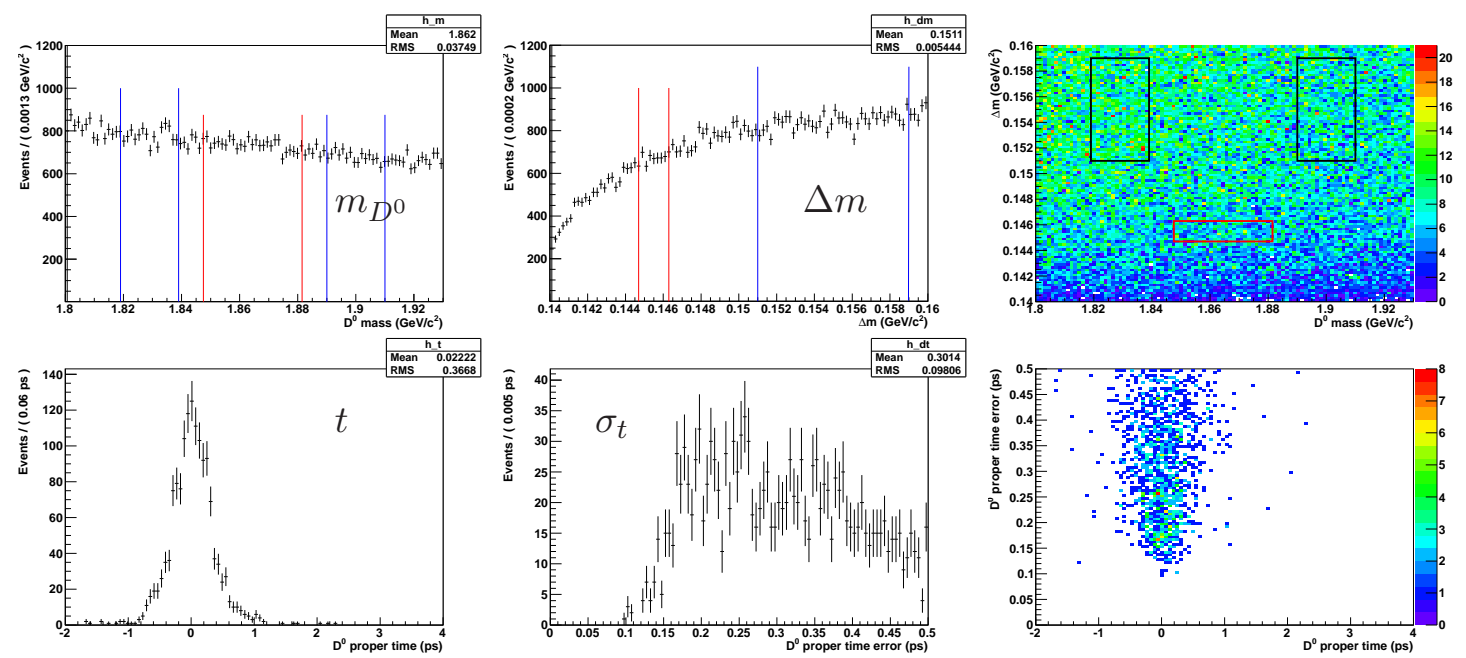

Figure E.12: Distributions of the tagged $K^{+} K^{-}$combinatorial truth-matched events. Top row: (left) $m_{D^{0}}$, (center) $\Delta m$ distribution and (right) 2 d scatted plot $\left(m_{D^{0}}, \Delta m\right)$. The signal region is indicated by a red line, the sidebands by a blue line. Bottom row: (left) $t$, (center) $\sigma_{t}$ distributions and (right) 2 d scatter plot $\left(t, \sigma_{t}\right)$ for events in the signal region. 

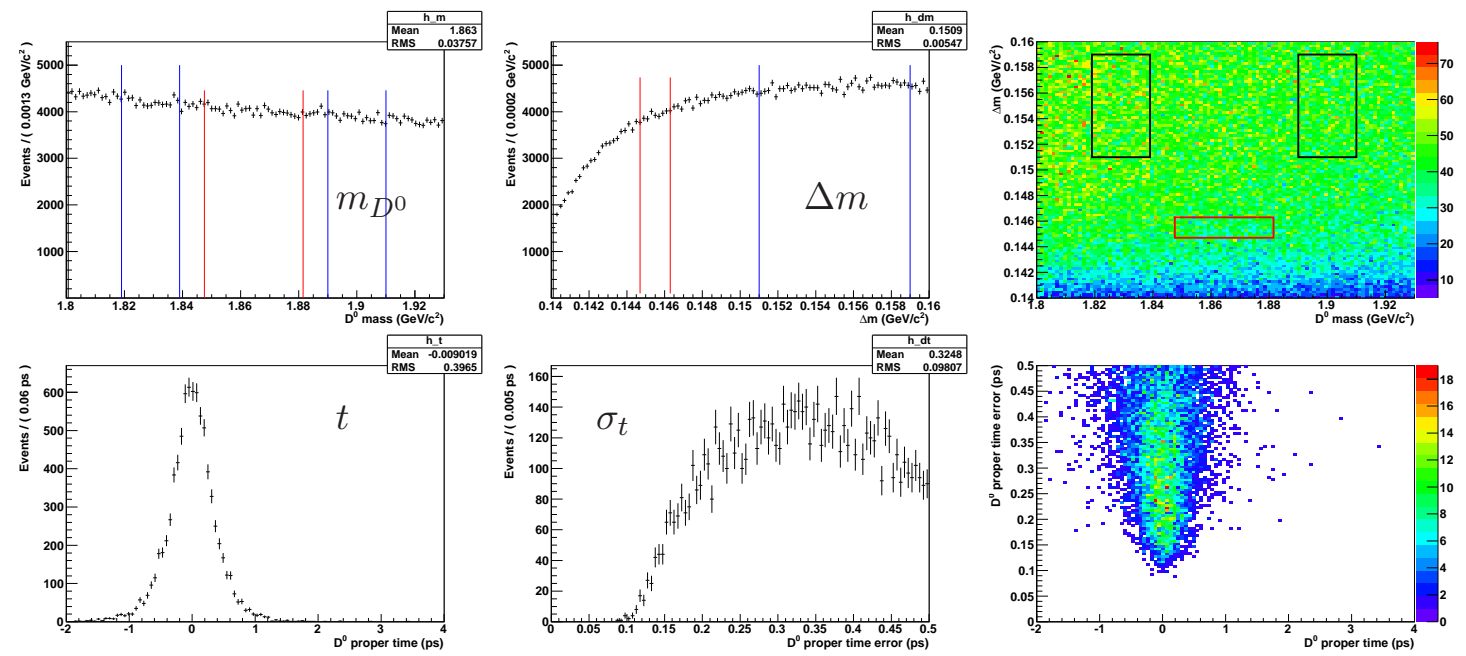

Figure E.13: Distributions of the tagged $K^{ \pm} \pi^{\mp}$ combinatorial truth-matched events. Top row: (left) $m_{D^{0}}$, (center) $\Delta m$ distribution and (right) 2 d scatted plot $\left(m_{D^{0}}, \Delta m\right)$. The signal region is indicated by a red line, the sidebands by a blue line. Bottom row: (left) $t$, (center) $\sigma_{t}$ distributions and (right) $2 \mathrm{~d}$ scatter plot $\left(t, \sigma_{t}\right)$ for events in the signal region.
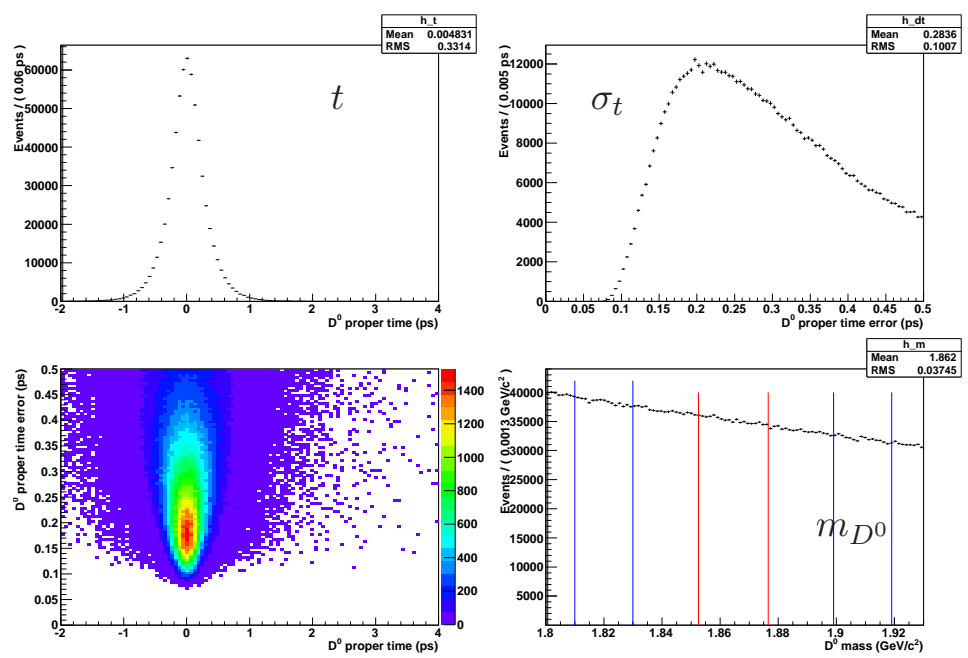

Figure E.14: Distributions of the untagged $K^{+} K^{-}$combinatorial truth-matched events. Top row: (left) $t$ and (right) $\sigma_{t}$ distributions for events in the signal region. Bottom row: (left) $2 \mathrm{~d}$ scatter plot $\left(t, \sigma_{t}\right)$ for the events in the signal region; (right) $m_{D^{0}}$ distribution, the signal region is indicated by a red line, the sidebands by a blue line. 

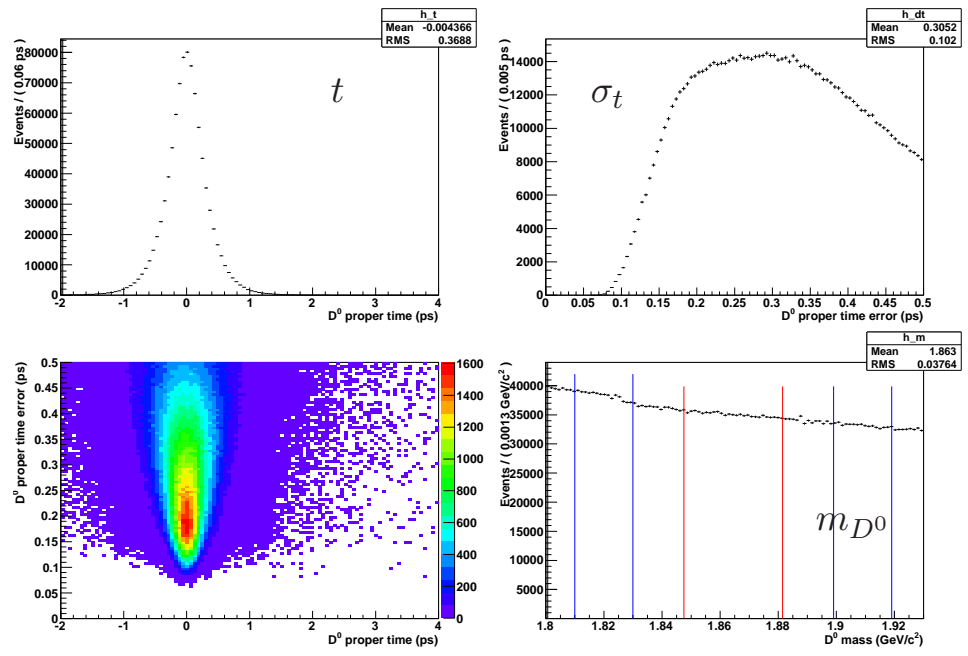

Figure E.15: Distributions of the untagged $K^{ \pm} \pi^{\mp}$ combinatorial truth-matched events. Top row: (left) $t$ and (right) $\sigma_{t}$ distributions for events in the signal region. Bottom row: (left) $2 \mathrm{~d}$ scatter plot $\left(t, \sigma_{t}\right)$ for the events in the signal region; (right) $m_{D^{0}}$ distribution, the signal region is indicated by a red line, the sidebands by a blue line. 


\section{Appendix F}

\section{Complete Fit Results}

In the following we report the values of the parameters extracted from the simultaneous fit to data.

The extracted resolution offset is $t_{0}=(-2.51 \pm 0.19) \mathrm{fs}$, while extracted lifetimes are:

$$
\begin{aligned}
\tau^{+} & =(405.69 \pm 1.25) \mathrm{fs} \\
\bar{\tau}^{+} & =(406.40 \pm 1.25) \mathrm{fs} \\
\tau_{K \pi} & =(408.97 \pm 0.24) \mathrm{fs} .
\end{aligned}
$$

The three Gaussians are weighted with the following fractions in the resolution function:

$$
\begin{aligned}
f_{1} & =0.192 \pm 0.018 \\
f_{21} & =0.00682 \pm 0.00086
\end{aligned}
$$

The scale factors of each Gaussian in the resolution model are:

$$
\begin{aligned}
& s_{1}=1.436 \pm 0.028 \\
& s_{2}=3.437 \pm 0.097 \\
& s_{3}=0.9411 \pm 0.0056,
\end{aligned}
$$

while the additional scale factors that take into account differences in resolution due to the final state or the $D^{0}$ momentum are:

$$
\begin{aligned}
S_{\pi \pi} & =0.9969 \pm 0.0068 \\
S_{K K} & =0.9959 \pm 0.0027 \\
S_{\mathrm{tag}}^{\prime} & =0.9982 \pm 0.0014
\end{aligned}
$$


The number of combinatorial events in the untagged $K^{+} K^{-}$mode is:

$$
N_{\text {comb }}\left(\operatorname{unt} K^{+} K^{-}\right)=164970 \pm 1000 .
$$

In Tab. F.1 we report the number of signal events for each mode and the number of combinatorial events in the untagged $K^{+} K^{-}$channel.

\begin{tabular}{r|r|r|r|r|r}
\hline \hline \multirow{2}{*}{$N_{\text {sig }}$} & \multicolumn{3}{|c|}{ tagged } & \multicolumn{2}{|c}{ untagged } \\
& $\pi^{+} \pi^{-}$ & $K^{+} K^{-}$ & $K^{ \pm} \pi^{\mp}$ & $K^{+} K^{-}$ & $K^{ \pm} \pi^{\mp}$ \\
\hline$D^{0}$ & $32690 \pm 190$ & $68820 \pm 260$ & 1487000 & 496200 & 5825300 \\
$\bar{D}^{0}$ & $32740 \pm 190$ & $68050 \pm 260$ & \pm 1200 & \pm 1200 & \pm 2600 \\
\hline \hline
\end{tabular}

Table F.1: Extracted numbers of signal events in the seven modes.

The values that are fixed in the fit are reported in the following.

The fraction of $D^{0}$ decays in the untagged $K^{+} K^{-}$channel is fixed to $f_{D^{0}}=0.5$, and the scale factors $S_{K \pi}=S_{\text {unt }}^{\prime}=1$ are also fixed in the nominal fit.

The fixed values regarding the PDFs of the three tagged channels and the untagged $K^{ \pm} \pi^{\mp}$ channel are reported in Tab. F.2.

The fixed values regarding the untagged $K^{+} K^{-}$PDFs are reported in Tab. F.3. For this channel, the number of misreconstructed-charm events in the signal regioni is fixed to $N_{\text {chr }}=5478$ and the weighting parameter of the combinatorial PDF is fixed to $h_{\text {comb }}=0.497$. 


\begin{tabular}{|c|c|c|c|c|}
\hline parameter & tagged $\pi^{+} \pi^{-}$ & tagged $K^{+} K^{-}$ & tagged $K^{ \pm} \pi^{\mp}$ & untagged $K^{ \pm} \pi^{\mp}$ \\
\hline & \multicolumn{4}{|c|}{ signal } \\
\hline$f_{\mathrm{tag}}^{ \pm}$ & $0.2 \%$ & $0.2 \%$ & - & - \\
\hline & \multicolumn{4}{|c|}{ misreconstructed-charm background } \\
\hline $\begin{array}{c}f_{\mathrm{crm}} \\
s_{1 \mathrm{crm}} \\
s_{2 \mathrm{crm}} \\
t_{0}^{1 \mathrm{crm}}(\mathrm{ps}) \\
t_{0}^{2 \mathrm{crm}}(\mathrm{ps}) \\
\tau_{1 \mathrm{crm}}(\mathrm{ps}) \\
\tau_{2 \mathrm{crm}}(\mathrm{ps}) \\
D^{*+} N_{\mathrm{chr}} \\
D^{*-} N_{\mathrm{chr}} \\
\end{array}$ & $\begin{array}{c}1 \\
1.35 \\
- \\
-1.96 \times 10^{-2} \\
- \\
0.457 \\
- \\
50 \\
47 \\
\end{array}$ & $\begin{array}{c}1 \\
1.24 \\
- \\
-2.2 \times 10^{-2} \\
- \\
0.437 \\
- \\
154 \\
156 \\
\end{array}$ & $\begin{array}{c}1 \% \\
1.07 \\
0.984 \\
1.1 \\
0.0 \\
1.50 \\
0.431 \\
642\end{array}$ & $\begin{array}{c}8.2 \% \\
2.19 \\
0.981 \\
0.25 \\
-2.5 \times 10^{-3} \\
0.992 \\
0.428 \\
4645\end{array}$ \\
\hline & \multicolumn{4}{|c|}{ combinatorial background } \\
\hline $\begin{array}{c}h_{\mathrm{comb}} \\
D^{*+} N_{\mathrm{comb}} \\
D^{*-} N_{\mathrm{comb}}\end{array}$ & $\begin{array}{l}0.496 \\
1830 \\
1830 \\
\end{array}$ & $\begin{array}{c}0.518 \\
274 \\
279 \\
\end{array}$ & $\begin{array}{l}0.542 \\
2849\end{array}$ & $\begin{array}{c}0.520 \\
1044600\end{array}$ \\
\hline
\end{tabular}

Table F.2: Fixed parameters in the final fit to data for the PDFs of the three categories of the tagged $\pi^{+} \pi^{-}, K^{+} K^{-}$and $K^{ \pm} \pi^{\mp}$ channels and for the untagged $K^{ \pm} \pi^{\mp}$ channel.

\begin{tabular}{c|cc|c}
\hline \hline \multirow{2}{*}{ parameter } & \multicolumn{2}{|c|}{ combinatorial PDF } & $\begin{array}{c}\text { misreconstucted } \\
\text { low SB }\end{array}$ \\
& high SB & charm \\
\hline$f_{1}$ & $0.81 \%$ & $0.95 \%$ & $7.9 \%$ \\
$f_{21}$ & $81.1 \%$ & $82.4 \%$ & - \\
$s_{1}$ & 3.09 & 3.16 & 2.90 \\
$s_{2}$ & 0.96 & 0.97 & 0.95 \\
$s_{3}$ & 1.50 & 1.49 & - \\
$t_{0}^{1}(\mathrm{ps})$ & 0.812 & 0.590 & -0.108 \\
$t_{0}^{2}(\mathrm{ps})$ & 0.024 & 0.024 & 0.008 \\
$t_{0}^{3}(\mathrm{ps})$ & 0.123 & 0.122 & - \\
$\tau_{1}(\mathrm{ps})$ & 0.814 & 0.758 & 1.36 \\
$\tau_{2}(\mathrm{ps})$ & 0.031 & 0.028 & 0.476 \\
$\tau_{3}(\mathrm{ps})$ & 0.146 & 0.131 & - \\
\hline \hline
\end{tabular}

Table F.3: Fixed parameters in the final fit to data for the PDFs of the background categories of the untagged $K^{+} K^{-}$channel. 


\section{Bibliography}

[1] M. Gell-Mann and A. Pais. Behavior of neutral particles under charge conjugation. Phys.Rev., 97:1387-1389, 1955.

[2] K. Lande, E.T. Booth, J. Impeduglia, L.M. Lederman, and W. Chinowsky. Observation of Long-Lived Neutral V Particles. Phys.Rev., 103:1901-1904, 1956.

[3] T.D. Lee and Chen-Ning Yang. Question of Parity Conservation in Weak Interactions. Phys.Rev., 104:254-258, 1956.

[4] C.S. Wu, E. Ambler, R.W. Hayward, D.D. Hoppes, and R.P. Hudson. Experimental Test of Parity Conservation in Beta Decay. Phys.Rev., 105:1413-1414, 1957.

[5] R.L. Garwin, L.M. Lederman, and M. Weinrich. Observations of the Failure of Conservation of Parity and Charge Conjugation in Meson Decays: The Magnetic Moment of the Free Muon. Phys.Rev., 105:1415-1417, 1957.

[6] T.D. Lee, R. Oehme, and C.-N. Yang. Remarks on Possible Noninvariance Under Time Reversal and Charge Conjugation. Phys.Rev., 106:340-345, 1957.

[7] J.H. Christenson, J.W. Cronin, V.L. Fitch, and R. Turlay. Evidence for the 2 pi Decay of the k(2)0 Meson. Phys.Rev.Lett., 13:138-140, 1964.

[8] L. Wolfenstein. Violation of CP Invariance and the Possibility of Very Weak Interactions. Phys.Rev.Lett., 13:562-564, 1964.

[9] H. Burkhardt et al. First Evidence for Direct CP Violation. Phys.Lett., B206:169, 1988.

[10] V. Fanti et al. A New measurement of direct CP violation in two pion decays of the neutral kaon. Phys.Lett., B465:335-348, 1999.

[11] A. Alavi-Harati et al. Observation of direct CP violation in $K_{(S, L)} \rightarrow \pi \pi$ decays. Phys.Rev.Lett., 83:22-27, 1999.

[12] M. Kobayashi and T. Maskawa. CP Violation in the Renormalizable Theory of Weak Interaction. Prog.Theor.Phys., 49:652-657, 1973. 
[13] M. K. Gaillard, B. W. Lee, and J. L. Rosner. Search for Charm. Rev.Mod.Phys., 47:277-310, 1975.

[14] H. Albrecht et al. Observation of $B^{0}-\bar{B}^{0}$ Mixing. Phys.Lett., B192:245, 1987.

[15] C. Albajar et al. Search for $B^{0}-\bar{B}^{0}$ Oscillations at the CERN Proton - anti-Proton Collider. 2. Phys.Lett., B186:247, 1987.

[16] A. Abulencia et al. Observation of $B_{s}-\bar{B}_{s}$ Oscillations. Phys.Rev.Lett., 97:242003, 2006.

[17] B. Aubert et al. Evidence for $D^{0}-\bar{D}^{0}$ Mixing. Phys. Rev. Lett., 98:211802, 2007.

[18] M. Staric et al. Evidence for $D^{0}-\bar{D}^{0}$ Mixing. Phys.Rev.Lett., 98:211803, 2007.

[19] T. Aaltonen et al. Evidence for $D^{0}-\bar{D}^{0}$ mixing using the CDF II Detector. Phys.Rev.Lett., 100:121802, 2008.

[20] R. Aaij et al. Evidence for CP violation in time-integrated $D^{0} \rightarrow h^{-} h^{+}$decay rates. Phys.Rev.Lett., 108:111602, 2012.

[21] G. Aad et al. Observation of a new particle in the search for the Standard Model Higgs boson with the ATLAS detector at the LHC. Phys.Lett., B716:1-29, 2012.

[22] S. Chatrchyan et al. Combined results of searches for the standard model Higgs boson in pp collisions at $\sqrt{(s)}=7 \mathrm{TeV}$. Phys.Lett., B710:26-48, 2012.

[23] K. Nakamura et al. Review of particle physics. J.Phys.G, G37:075021, 2010.

[24] L. Wolfenstein. Parametrization of the Kobayashi-Maskawa Matrix. Phys.Rev.Lett., $51: 1945,1983$.

[25] J. Charles et al. CP violation and the CKM matrix: Assessing the impact of the asymmetric B factories. Eur.Phys.J., C41:1-131, 2005.

[26] I. I. Bigi and A. I. Sanda. CP violation. Camb. Monogr. Part. Phys. Nucl. Phys. Cosmol., pages 1-485, 2009.

[27] A. Alavi-Harati et al. A Measurement of the K(L) charge asymmetry. Phys.Rev.Lett., $88: 181601,2002$.

[28] Ikaros I. Y. Bigi and Nikolai G. Uraltsev. $D^{0}-\bar{D}^{0}$ oscillations as a probe of quarkhadron duality. Nucl. Phys., B592:92-106, 2001.

[29] A. F. Falk, Y. Grossman, Z. Ligeti, Y. Nir, and A. A. Petrov. The $D^{0}-\bar{D}^{0}$ Mass Difference from a Dispersion Relation. Phys. Rev., D69:114021, 2004.

[30] A. A Petrov. Charm mixing in the standard model and beyond. Int. J. Mod. Phys., A21:5686-5693, 2006. 
[31] S. Bergmann, Y. Grossman, Z. Ligeti, Y. Nir, and A. A. Petrov. Lessons from CLEO and FOCUS measurements of $D^{0}-\bar{D}^{0}$ mixing parameters. Phys. Lett., B486:418-425, 2000 .

[32] http://www.slac.stanford.edu/xorg/hfag/index.html.

[33] B. Aubert et al. The BABAR Detector. Nucl. Instrum. Meth., A479:1-116, 2002.

[34] PEP-II: An Asymmetric B Factory. Conceptual Design Report. 1994.

[35] P. F. Harrison and Helen R. Quinn. The BABAR Physics Book: Physics at an Asymmetric B Factory, Chap. 3, Table 3-1, p. 75. Papers from Workshop on Physics at an Asymmetric B Factory (BaBar Collaboration Meeting), Rome, Italy, 11-14 Nov 1996, Princeton, NJ, 17-20 Mar 1997, Orsay, France, 16-19 Jun 1997 and Pasadena, CA, 22-24 Sep 1997.

[36] J. Seeman, M. Browne, Y. Cai, W. Colocho, F.-J. Decker, et al. A Luminosity of $10^{34} \mathrm{~cm}^{-2} \mathrm{~s}^{-1}$ in the PEP-II B-Factory. Conf.Proc., C060626:643-645, 2006.

[37] C. Bozzi et al. The BABAR Silicon Vertex Tracker. Nucl.Instrum.Meth., A435:25-33, 1999.

[38] V. Re, C. Borean, C. Bozzi, V. Carassiti, A. Cotta-Ramusino, et al. Performance of the BABAR Silicon Vertex Tracker. Nucl.Instrum.Meth., A501:14-21, 2003.

[39] G. Sciolla et al. The BABAR Drift Chamber. Nucl.Instrum.Meth., A419:310-314, 1998.

[40] Michael H. Kelsey. Performance and aging of the BABAR drift chamber. Nucl.Instrum.Meth., A535:206-211, 2004.

[41] I. Adam et al. DIRC, the internally reflecting ring imaging Cherenkov detector for BABAR. IEEE Trans.Nucl.Sci., 45:657-664, 1998.

[42] B. Lewandowski. The BABARelectromagnetic calorimeter. Nucl.Instrum.Meth., A494:303-307, 2002.

[43] R. Santonico and R. Cardarelli. Development of Resistive Plate Counters. Nucl.Instrum.Meth., 187:377-380, 1981.

[44] F. Anulli, A. Asmone, R. Baldini Ferroli, K. van Bibber, R.M. Bionta, et al. The muon and neutral hadron detector for BABAR. Nucl.Instrum.Meth., A409:542-546, 1998.

[45] W. Menges et al. The BABAR muon system upgrade. IEEE Nucl.Sci.Symp.Conf.Rec., 5:1470-1474, 2006.

[46] G. Battistoni, E. Iarocci, M.M. Massai, G. Nicoletti, and L. Trasatti. Operation of Limited Streamer Tubes. Nucl.Instrum.Meth., 164:57, 1979. 
[47] F. Anulli, R. Baldini, A. Calcaterra, L. Daniello, R. de Sangro, et al. BABAR forward endcap upgrade. Nucl.Instrum.Meth., A539:155-171, 2005.

[48] Torbjorn Sjostrand et al. High-energy-physics event generation with pythia 6.1. Comput. Phys. Commun., 135:238-259, 2001.

[49] S. Agostinelli et al. Geant4: A simulation toolkit. Nucl. Instrum. Meth., A506:250303, 2003.

[50] Bernard Aubert et al. Measurement of $D^{0}-\bar{D}^{0}$ mixing using the ratio of lifetimes for the decays $D^{0} \rightarrow K^{-} \pi^{+}, K^{-} K^{+}$, and $\pi^{-} \pi^{+}$. Phys.Rev., D78:011105, 2008.

[51] B. Aubert et al. Measurement of $D^{0}-\bar{D}^{0}$ Mixing using the Ratio of Lifetimes for the Decays $D^{0} \rightarrow K^{-} \pi^{+}$and $K^{+} K^{-}$. Phys. Rev., D80:071103, 2009.

[52] L. Lyons, D. Gibaut, and P. Clifford. How to combine correlated estimates of a single physical quantity. Nucl. Instrum. Meth., A270:110, 1988.

[53] S. Baker and R. D. Cousins. Clarification of the use of chi square and likelihood functions in fits to histograms. Nucl. Instrum. Meth., 221:437-442, 1984.

[54] G. Punzi. Comments on Likelihood fits with variable resolution, arXiv:physics/0401045, January 2004.

[55] W. Verkerke and D. Kirkby. RooFit Users Manual v2.91, ftp://root.cern.ch/root/doc/RooFit_Users_Manual_2.91-33.pdf, Oct., 2008.

[56] CHARM2012. The $5^{\text {th }}$ International Workshop on Charm Pysics. 14-17 May 2012, Honolulu, Hawai'i, May 2012.

[57] T. Aaltonen et al. Measurement of CP-violating asymmetries in $D^{0} \rightarrow \pi^{+} \pi^{-}$and $D^{0} \rightarrow K^{+} K^{-}$decays at CDF. Phys.Rev., D85:012009, 2012.

[58] J.P. Lees et al. Measurement of $D^{0}-\bar{D}^{0}$ Mixing and $C P$ Violation in Two-Body $D^{0}$ Decays. arXiv:hep-ex/1209.3896, September 2012.

[59] P. del Amo Sanchez et al. Measurement of $D^{0}-\bar{D}^{0}$ mixing parameters using $D^{0} \rightarrow$ $K_{S}^{0} \pi^{+} \pi^{-}$and $D^{0} \rightarrow K_{S}^{0} K^{+} K^{-}$decays. Phys.Rev.Lett., 105:081803, 2010.

[60] C. Jarlskog. Commutator of the Quark Mass Matrices in the Standard Electroweak Model and a Measure of Maximal CP Violation. Phys.Rev.Lett., 55:1039, 1985. 